\title{
Development of an Artificial Neural Network to Predict In-Use Engine Emissions
}

\author{
Melissa L. Morris \\ West Virginia University
}

Follow this and additional works at: https://researchrepository.wvu.edu/etd

\section{Recommended Citation}

Morris, Melissa L., "Development of an Artificial Neural Network to Predict In-Use Engine Emissions" (2011). Graduate Theses, Dissertations, and Problem Reports. 3086.

https://researchrepository.wvu.edu/etd/3086

This Dissertation is protected by copyright and/or related rights. It has been brought to you by the The Research Repository @ WVU with permission from the rights-holder(s). You are free to use this Dissertation in any way that is permitted by the copyright and related rights legislation that applies to your use. For other uses you must obtain permission from the rights-holder(s) directly, unless additional rights are indicated by a Creative Commons license in the record and/ or on the work itself. This Dissertation has been accepted for inclusion in WVU Graduate Theses, Dissertations, and Problem Reports collection by an authorized administrator of The Research Repository @ WVU.

For more information, please contact researchrepository@mail.wvu.edu. 


\title{
Development of an Artificial Neural Network to Predict In-Use Engine Emissions
}

\author{
Submitted by: \\ Melissa L. Morris
}

Dissertation submitted to the College of Engineering and Mineral Resources

at West Virginia University

in partial fulfillment of the requirements for the degree of

Doctor of Philosophy in Mechanical Engineering

\author{
Committee Members: \\ Dr. John Nuszkowski, Committee Chairperson \\ Dr. Kenneth Means \\ Dr. W. Scott Wayne \\ Dr. Gregory Thompson \\ Mr. Samuel Ameri
}
Mechanical and Aerospace Engineering Department
Morgantown, WV 26505-6106

Keywords: Artificial Neural Network, Emissions Prediction, Road Grade

Copyright 2011 


\title{
Abstract \\ Development of an Artificial Neural Network to Predict In-Use Engine Emissions
}

Melissa L. Morris

\begin{abstract}
A method to predict in-use diesel engine emissions is developed based on engine dynamometer and in-use data acquired at the West Virginia University Center for Alternative Fuels, Engines, and Emissions. (WVU CAFEE). The model accounts for the effects of road grade on generated emissions; a need for this model is evident in literature. Current modeling methods do not account for the effects of road grade, and have been shown to under-predict NOx by as much as $57 \%$. It is determined through present research and a review of relevant literature that an artificial neural network (ANN) was the most applicable modeling method.
\end{abstract}

A modular ANN was developed to predict the heavy duty diesel engine emissions. The two modules were trained independently, the first module was trained with data acquired through inuse testing, and the second module was trained with data acquired via engine dynamometer testing. The first module predicted the engine speed and torque associated with the inputs of road grade and vehicle speed, while the second ANN employed the first ANN's outputs, and predicts the emitted quantities of $\mathrm{NOx}, \mathrm{CO}_{2}, \mathrm{HC}$, and $\mathrm{CO}$. A series of training and verification runs are conducted in order to determine the optimum ANN characteristics. Once the ANN was finalized, it was trained with and employed to predict the emissions associated with a variety of routes.

When the ANN was trained with a combination of in-use and engine dynamometer data, the ANN is able to predict NOx emissions associated with that same route within $6 \%$ of the measured values. The average difference between the measured and predicted $\mathrm{CO}_{2}$ values for the same training and verification scenario mentioned above was less than $15 \%$. It was also demonstrated that the ANN was able to predict emissions that are associated with routes that differ from those by which it is trained. When the ANN was trained with in-use data from a specific route, it was able to predict the $\mathrm{NOx}$ and $\mathrm{CO}_{2}$ emissions associated with a different route with percent differences from the measured values of $20 \%$ or less. 


\section{Dedication}

This dissertation is dedicated to my father; he inspired me to be where I am today. I hope one day to be as well respected and successful as he is as a faculty member, a parent, and a person in general. 


\section{Acknowledgements}

I would like to thank my advisor, Dr. John Nuszkowski, for being the best advisor ever! I am also grateful to my committee members, Dr. Gregory Thompson, Dr. Kenneth Means, Dr. W. Scott Wayne, and Mr. Samuel Ameri for their time and input. I would like to express a special thanks to Dr. Thompson for always being there to listen to my rants and rambles and encouraging me along the way. Dr. Means also deserves special recognition for supporting me through the duration of my graduate school experience and providing me with opportunities to work with students through his senior design courses.

I also would like to thank my parents, Gary and Lynn Morris, for being supportive and encouraging throughout my education. I especially thank them for instilling their values in me, and molding me into the person I am today.

My former students also deserve recognition; they are the main source of motivation for my pursuit of a doctorate degree. One student in particular, Justin Heydon, served as an inspiration and has provided me with constant encouragement and friendship over the past year.

I also would like to thank Jackson Wolfe, I am grateful to Jackson for always making me smile, for all of the great times we have had, and for those yet to come. 


\section{Table of Contents}

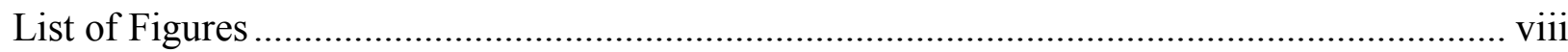

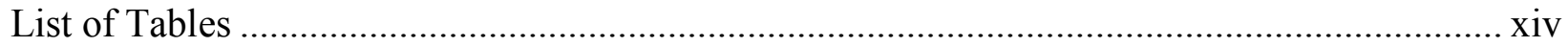

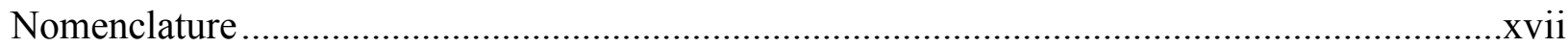

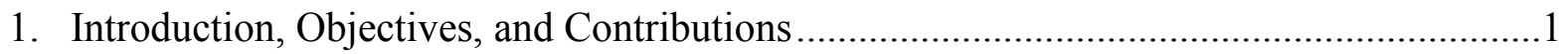

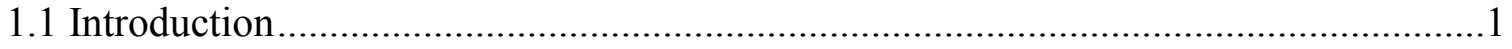

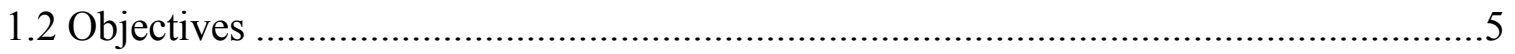

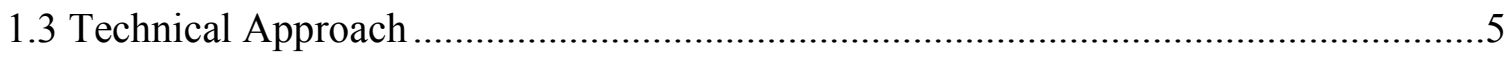

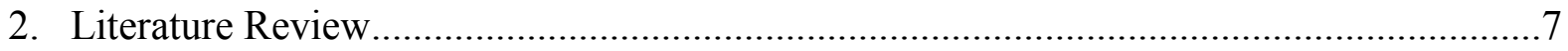

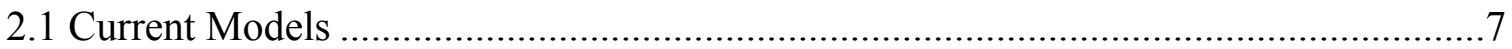

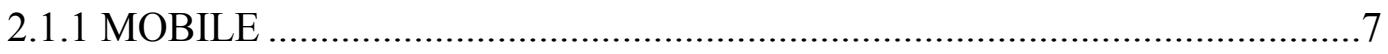

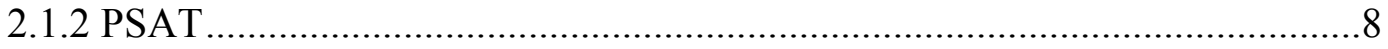

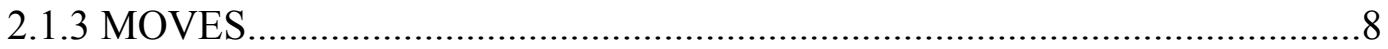

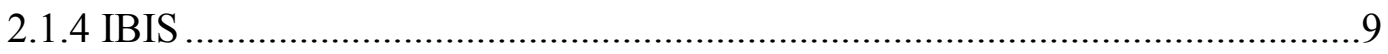

2.1.5 Accuracy of MOBILE Model ................................................................

2.1.6 Accuracy of EMFAC Model.................................................................11

2.1.7 Accuracy of Emissions Factor Models .........................................................11

2.1.8 Emissions Inventory Estimation .............................................................14

2.1.9 Modal Modeling Methods..........................................................................14

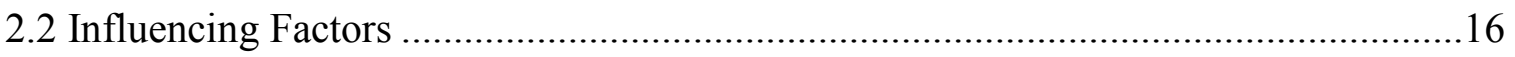

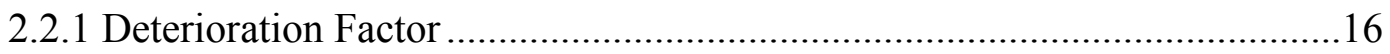

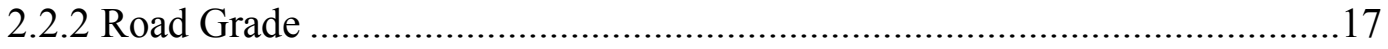

2.3 Defeat Devices and Consent Decrees ....................................................................

2.4 Artificial Intelligence Modeling Techniques ........................................................18

2.4.1 Expert Systems ....................................................................................18

2.4.2 Bayesian Networks ..............................................................................19

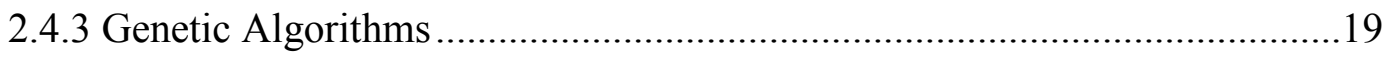

2.4.4 Artificial Neural Networks .................................................................20

2.4.5 ANN Applied to Emissions Modeling ......................................................24 


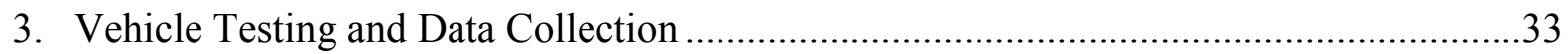

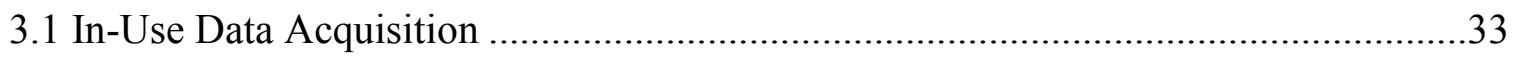

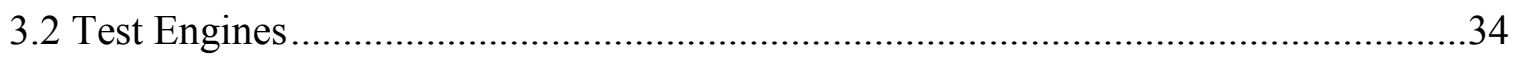

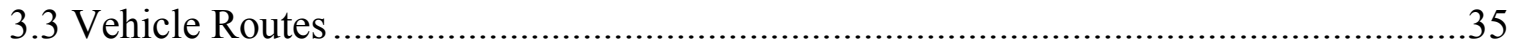

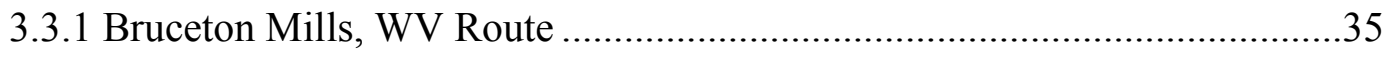

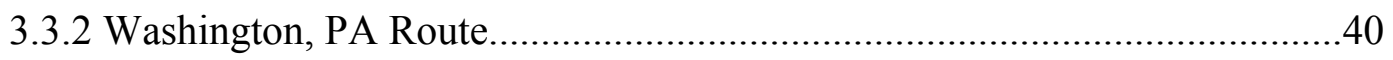

3.3.3 In-Use Data Set Designations ...........................................................49

3.4 Engine Dynamometer Data Acquisition ..........................................................50

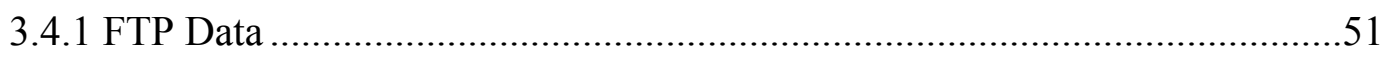

3.4.2 Bruceton Mills, WV Cycle .................................................................55

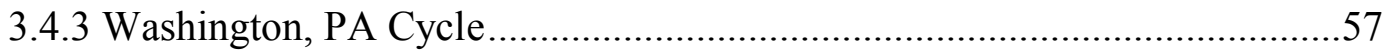

4. Repeatability of Measured Emissions Data ................................................................60

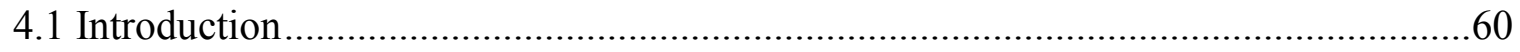

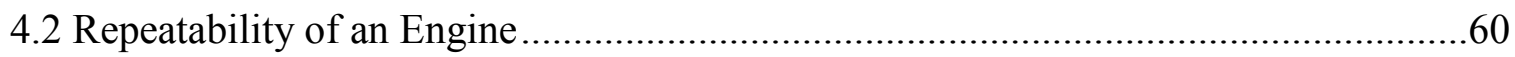

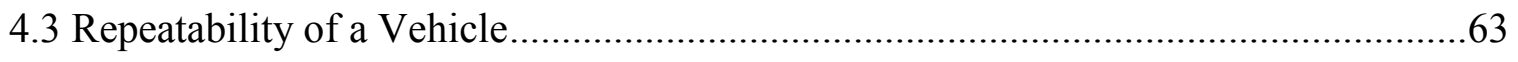

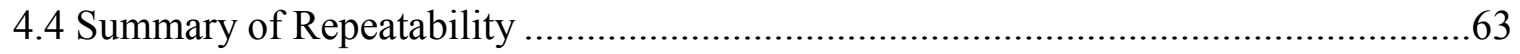

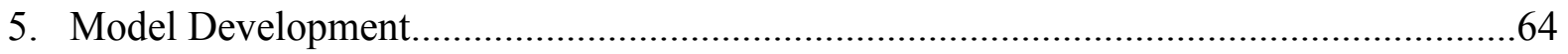

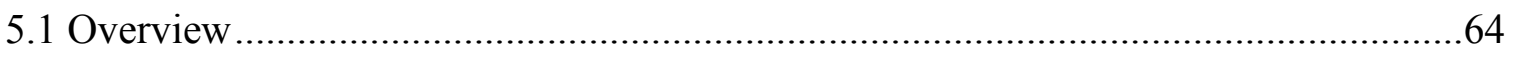

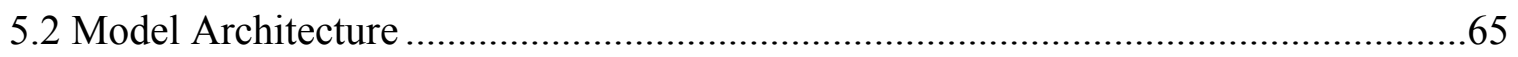

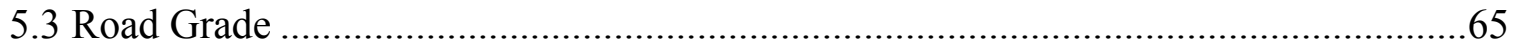

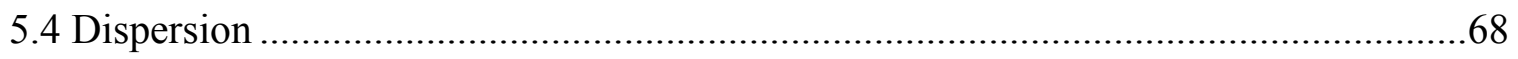

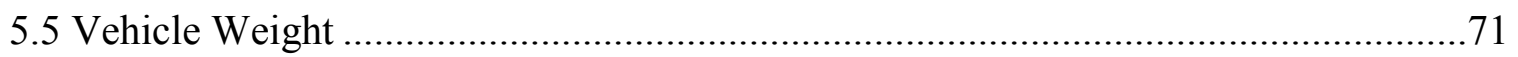

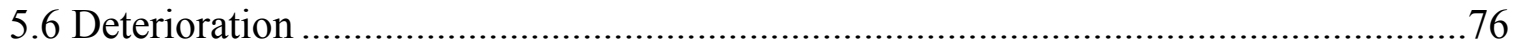

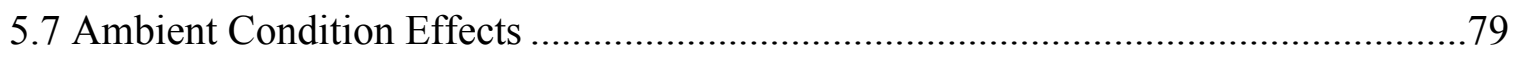

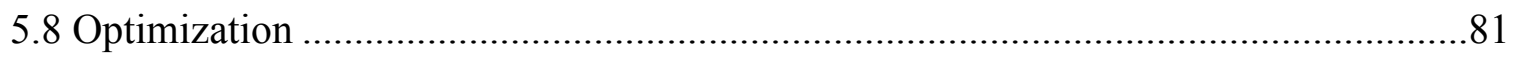

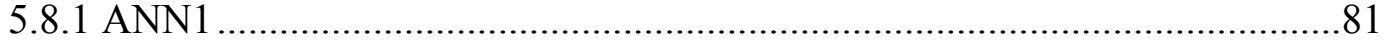

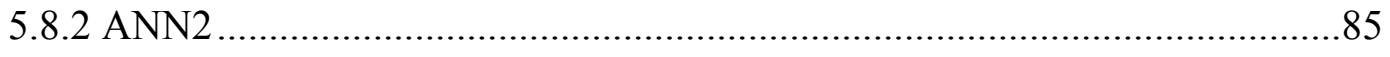

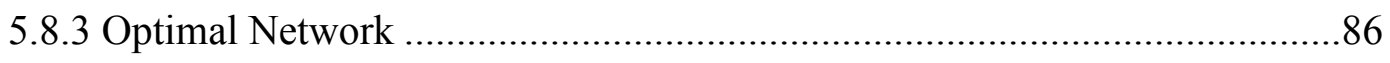

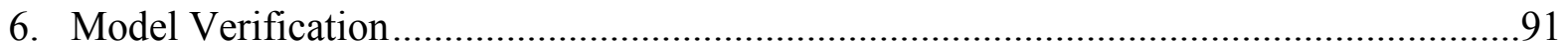

6.1 Repeated Bruceton Mills, WV Routes.............................................................91 


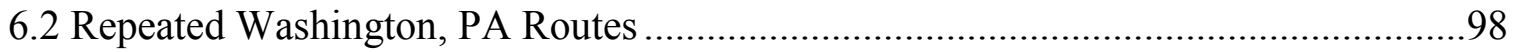

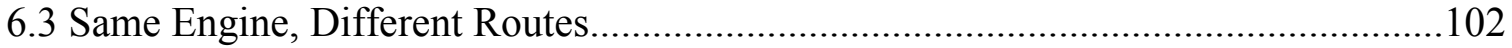

6.4 Same Route, Different Cycles....................................................................... 111

6.5 Comparison of Measured and Predicted Emissions to EPA Regulations ...................119

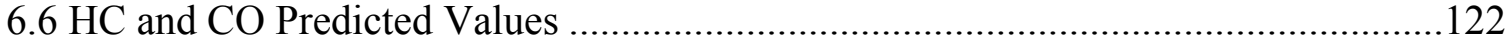

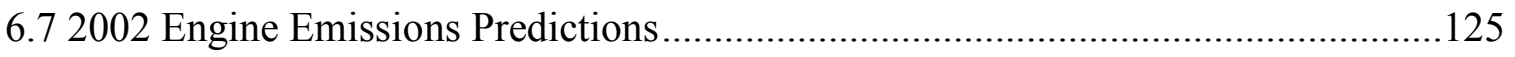

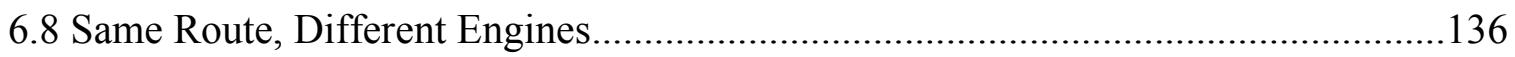

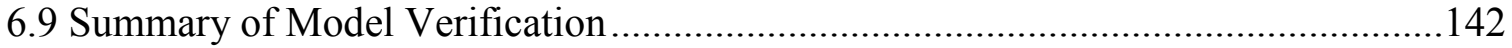

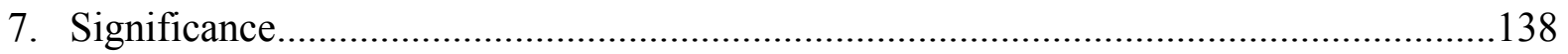

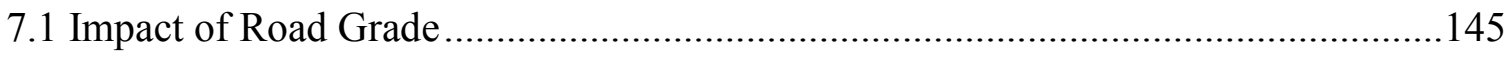

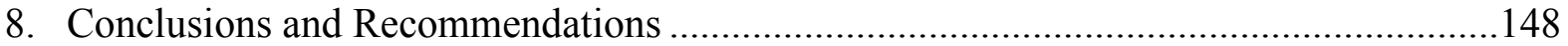

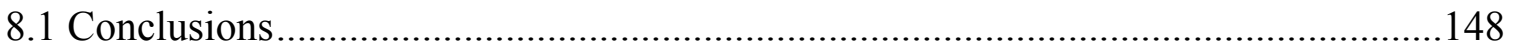

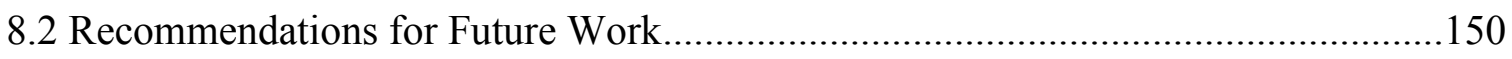

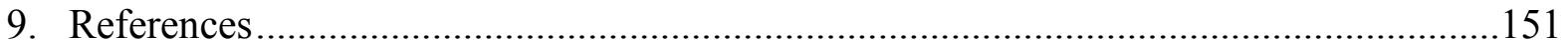

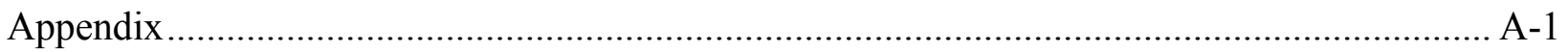




\section{List of Figures}

Figure 2.4.4.1: Schematic of Human Neuron ...................................................................21

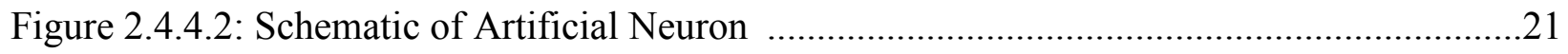

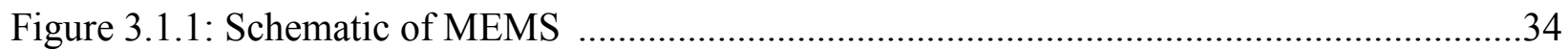

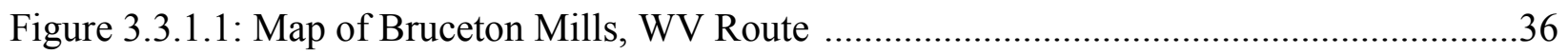

Figure 3.3.1.2: Engine Speed for the Sabraton, WV to Bruceton Mills, WV Route with

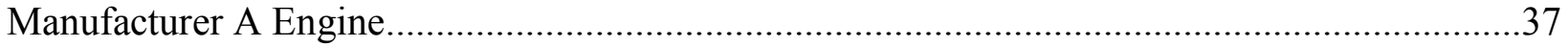

Figure 3.3.1.3: Engine Torque for the Sabraton, WV to Bruceton Mills, WV Route with

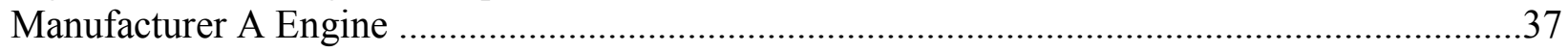

Figure 3.3.1.4: Road Grade from Sabraton, WV to Bruceton Mills, WV ..................................38

Figure 3.3.1.5: Engine Speed for Bruceton Mills, WV to Sabraton, WV Route with Manufacturer A Engine 39

Figure 3.3.1.6: Engine Torque for the Sabraton, WV to Bruceton Mills, WV Route with

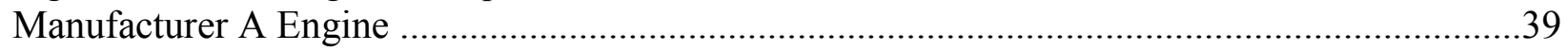

Figure 3.3.1.7: Road Grade for Bruceton Mills, WV to Sabraton, WV ....................................40

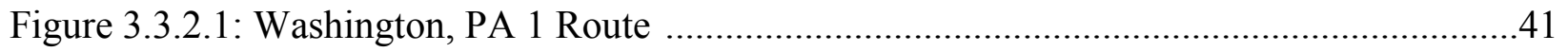

Figure 3.3.2.2: Engine Speed for Washington, PA 1 Route with Manufacturer A Engine ..........42

Figure 3.3.2.3: Engine Torque for Washington, PA1 Route with Manufacturer A Engine .........42

Figure 3.3.2.4: Road Grade for the Washington, PA 1 Route ................................................43

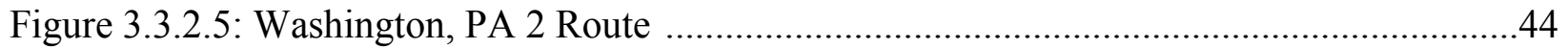

Figure 3.3.2.6: Engine Speed for Washington, PA 2 Route with Manufacturer A Engine ..........44

Figure 3.3.2.7: Engine Torque for Washington, PA 2 Route with Manufacturer A Engine ........45

Figure 3.3.2.8: Road Grade for Washington PA 2 Route ......................................................46

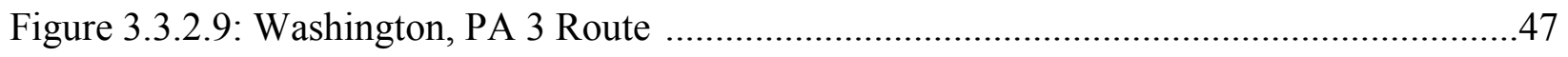

Figure 3.3.2.10: Engine Speed for Washington, PA 3 Route with Manufacturer B Engine ........47 
Figure 3.3.2.11: Engine Torque for Washington, PA 3 Route with Manufacturer B Engine .......48

Figure 3.3.2.12: Road Grade for Washington, PA 3 Route ...................................................49

Figure 3.4.1: Schematic of Test Setup at EERC .................................................................51

Figure 3.4.1.1: Manufacturer A Engine Speed During FTP Test ...........................................52

Figure 3.4.1.2: Manufacturer A Engine Torque During FTP Test .........................................52

Figure 3.4.1.3: NOx Comparison When Emissions Module was Trained with FTP Data ...........53

Figure 3.4.1.4: $\mathrm{CO}_{2}$ Comparison When Emissions Module was Trained with FTP Data ............54

Figure 3.4.1.5 NOx Emissions versus Horsepower for Bruceton Mills, WV and FTP Engine

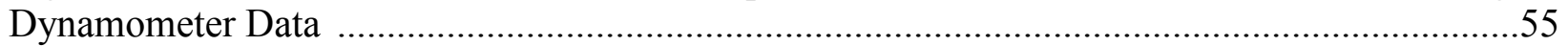

Figure 3.4.2.1 Engine Speed for Sabraton, WV to Bruceton Mills, WV Engine Dynamometer

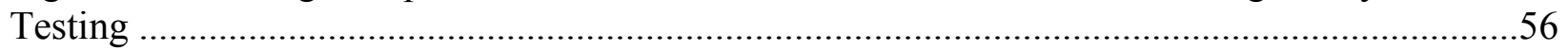

Figure 3.4.2.2: Engine Torque for Sabraton, WV to Bruceton Mills, WV Engine Dynamometer

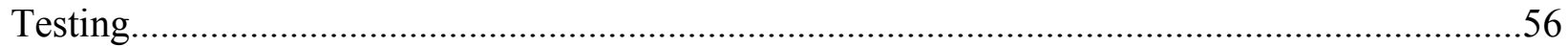

Figure 3.4.3.1: Engine Speed for Washington, PA Engine Dynamometer Testing .....................57

Figure 3.4.3.2: Engine Torque for Washington, PA Engine Dynamometer Testing ....................58

Figure 3.4.3.3: Engine Speed for Bruceton Mills, WV to Sabraton, WV Engine Dynamometer Testing .59

Figure 3.4.3.4: Engine Torque for Bruceton Mills, WV to Sabraton, WV Engine Dynamometer

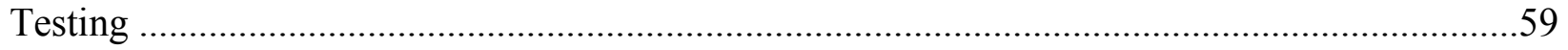

Figure 5.2.1: Basic Structure of Model ............................................................................65

Figure 5.3.1: Diagram of Relationship Between Distance Traveled and Altitude Change .........66

Figure 5.3.2: Height for Bruceton Mills, WV Route .......................................................67

Figure 5.3.3: Road Grade for Bruceton Mills, WV Route .....................................................67

Figure 5.5.1: Comparison of Predicted and Measured Engine Speed for Manufacturer B Engine

Figure 5.5.2: Comparison of Predicted and Measured Engine Torque for Manufacturer B Engine without Correcting for Weight Difference Between Training and Verification Vehicles ..... .75 
Figure 5.5.3: Comparison of Scaled and Predicted Engine Torque for Manufacturer B Engine when Correcting for Weight Difference Between Training and Verification Vehicles .75

Figure 5.6.1: NOx Emissions versus Odometer Reading .........................................................78

Figure 5.6.2: $\mathrm{CO}_{2}$ Emissions versus Odometer Reading ……….............................................79

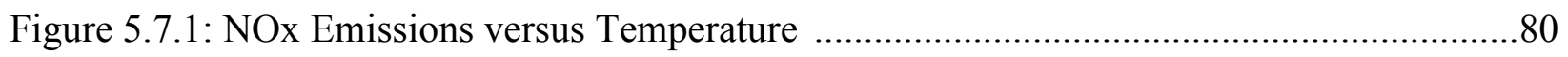

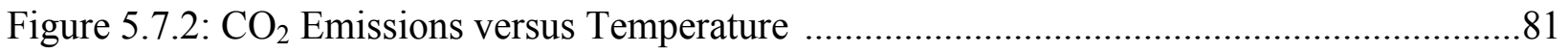

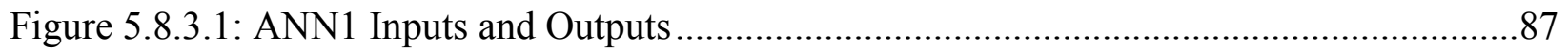

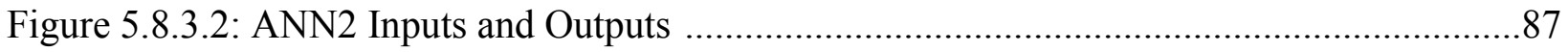

Figure 5.8.3.3: Tan-Sigmoid Transfer Function Plot …………............................................... 88

Figure 5.8.3.3: Vehicle ANN, ANN1, Neuron Layer Structure .................................................89

Figure 5.8.3.4: Emissions ANN, ANN2, Neuron Layer Structure ..........................................90

Figure 6.1.1: Vehicle ANN Predicted and Actual Engine Torque When Training with and Predicting Bruceton2Sab 2 Data Set ..................................................................................94

Figure 6.1.2: Vehicle ANN Predicted and Actual Engine Speed When Training with and

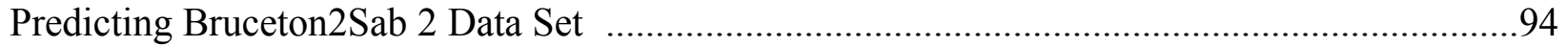

Figure 6.1.3: Difference Between Predicted and Measured Engine Speed for Run Sab2Bruceton 2 Verification Data .95

Figure 6.1.4: Difference Between Predicted and Measured Engine Torque for Run 1

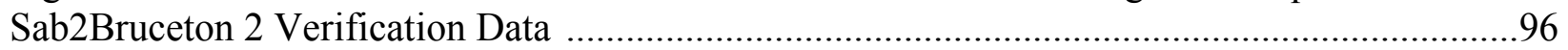

Figure 6.1.5: Difference Between Predicted and Measured NOx Emissions for Run 1

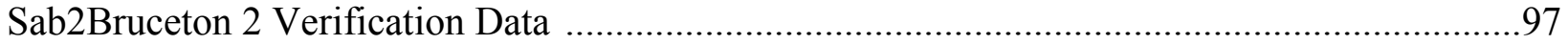

Figure 6.1.6: Difference Between Predicted and Measured $\mathrm{CO}_{2}$ Emissions for Run 1

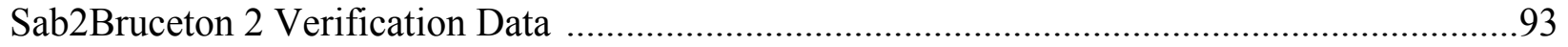

Figure 6.2.1: Difference Between Predicted and Measured Engine Speed for the Washington, PA

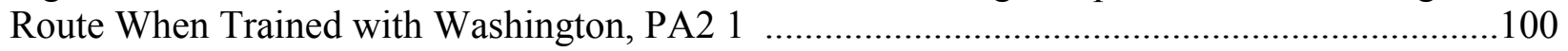

Figure 6.2.2: Difference Between Predicted and Measured Torque for the Washington, PA Route When Trained With Washington, PA2 1 100 
Figure 6.2.3: Difference Between Predicted and Measured NOx for the Washington, PA Route When Trained with Washington, PA2 1 and

Figure 6.2.4: Difference Between Predicted and Measured $\mathrm{CO}_{2}$ for the Washington, PA Route When Trained with Washington, PA2 1

Figure 6.3.1: Difference Between Predicted and Measured Engine Speed for the Sabraton, WV to Bruceton Mills, WV Route When ANN was Trained with Washington, PA2 1 .104

Figure 6.3.2: Difference Between Predicted and Measured Engine Torque for the Sabraton, WV to Bruceton Mills, WV Route When ANN was Trained with Washington, PA2 1 105

Figure 6.3.3: Difference Between Predicted and Measured NOx for the Sabraton, WV to Bruceton Mills, WV Route When ANN was Trained with Washington, PA2 1 .105

Figure 6.3.4: Difference Between Predicted and Measured $\mathrm{CO}_{2}$ for the Sabraton, WV to Bruceton Mills, WV Route When ANN was Trained with Washington, PA2 1 .106

Figure 6.3.5: Difference Between Predicted and Measured Engine Speed for the Bruceton2Sab 1 Route 107

Figure 6.3.6: Difference Between Predicted and Measured Engine Torque for the Bruceton2Sab 1 Route 109

Figure 6.3.7: Difference Between Predicted and Measured NOx for the Bruceton2Sab 1 Route 110

Figure 6.3.8: Difference Between Predicted and Measured $\mathrm{CO}_{2}$ for the Bruceton2Sab 1 Route 110

Figure 6.4.1: Difference Between Predicted and Measured Engine Speed for the Sab2Bruceton 1 Data Set. .112

Figure 6.4.2: Difference Between Predicted and Measured Engine Torque for the Sab2Bruceton 1 Data Set .113

Figure 6.4.3: Difference Between Predicted and Measured NOx for the Sab2Bruceton 1 Data Set 114

Figure 6.4.4: Difference Between Predicted and Measured $\mathrm{CO}_{2}$ for the Sab2Bruceton 1 Data Set .114

Figure 6.4.5: Difference Between Predicted and Measured Engine Speed for the Washington PA1 When ANN Was Trained With Washington PA2 1 Route. .116 
Figure 6.4.6: Difference Between Predicted and Measured Engine Torque for the Washington PA1 When ANN was Trained With Washington PA2 1 Route.

Figure 6.4.7: Difference Between Predicted and Measured $\mathrm{CO}_{2}$ Emissions for the Washington PA1 When ANN was Trained With Washington PA2 1 Route.

Figure 6.4.8: Difference Between Predicted and Measured NOx Emissions for the Washington PA1 When ANN was Trained With Washington PA2 1 Route. 118

Figure 6.7.1: : Predicted and Measured Engine Speed for Washington PA1 When the ANN was Trained with Data from Washington PA2 Route.

Figure 6.7.2: Predicted and Measured Torque for Washington PA1 When the ANN was Trained with Data from Washington PA2 Route .128

Figure 6.7.3: Predicted and Measured NOx Emissions for Washington PA1 When the ANN was Trained with Data from Washington PA2 Route.

Figure 6.7.4: Predicted and Measured CO2 Emissions for Washington PA1 When the ANN was Trained with Data from Washington PA2 Route. .129

Figure 6.7.5: Predicted and Measured Engine Speed for Sabraton, WV to Washington, PA When the ANN was Trained with Data from Washington, PA3 to Sabraton, WV Route. 130

Figure 6.7.6: Predicted and Measured Torque for Sabraton, WV to Washington, PA When the ANN was Trained with Data from Washington, PA3 to Sabraton, WV Route. 131

Figure 6.7.7: Predicted and Measured NOx Emissions for Sabraton, WV to Washington, PA When the ANN was Trained with Data from Washington, PA3 to Sabraton, WV Route .....

Figure 6.7.8: Predicted and Measured $\mathrm{CO}_{2}$ Emissions for Sabraton, WV to Washington, PA When the ANN was Trained with Data from Washington, PA3 to Sabraton, WV Route..... 132

Figure 6.7.9: Predicted and Measured NOx Emissions for Sabraton, WV to Washington, PA When the ANN was Trained with Data from Washington, PA3 to Sabraton, WV Route .134

Figure 6.7.10: Predicted and Measured $\mathrm{CO}_{2}$ Emissions for Sabraton, WV to Washington, PA When the ANN was Trained with Data from Washington, PA3 to Sabraton,WV Route .134

Figure 6.7.11: Predicted and Measured NOx Emissions for Sabraton, WV to Washington, PA When the ANN was Trained with Data from PA3 to Sabraton, WV Route. .135

Figure 6.7.12: Predicted and Measured $\mathrm{CO}_{2}$ Emissions for Washington, PA to Sabraton, WV When the ANN was Trained with Data from Sabraton, WV to Washington, PA Route 136 
Figure 6.8.1: Predicted and Measured NOx Emissions for Washington, PA to Sabraton, WV When the ANN was Trained with Data from Sabraton, WV to Washington, PA Route 138

Figure 6.8.2: Predicted and Measured $\mathrm{CO}_{2}$ Emissions for Bruceton Mills, WV route When the ANN was Trained with Data from Washingtonington, PA 2 Route

Figure 6.8.3: Predicted and Measured NOx Emissions for Bruceton Mills, WV route When the ANN was Trained with Data from Washington, PA 2 Route 141

Figure 6.8.4: Predicted and Measured $\mathrm{CO}_{2}$ Emissions for Washington, PA route When the ANN Was Trained with Data from Washington, PA 2 Route 141 


\section{List of Tables}

Table 1.1.1: Emissions Regulations .....................................................................................

Table 2.4.3.1: Genetic Algorithm Approach .................................................................20

Table 2.4.4.1: Commonly Used Transfer Functions in ANN .............................................24

Table 2.4.6.1: Engine Operating Parameters Used As Inputs to Genetic Algorithm ..................30

Table 3.2.1: Manufacturer A Engine Specifications ..........................................................

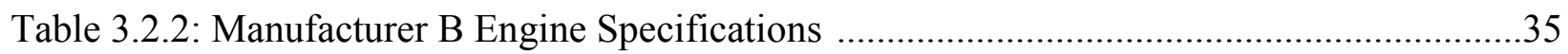

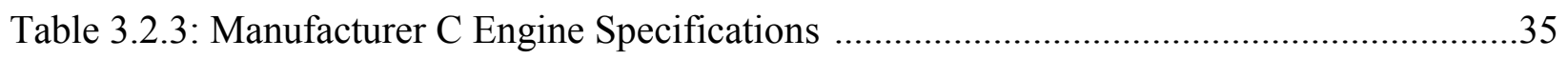

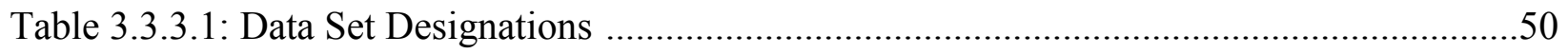

Table 4.2.1: Statistical Analysis of MEMS Data Summary for Manufacturer A Engine.............61

Table 4.2.2: Statistical Analysis of MEMS Data Summary for Manufacturer D Engine.............62

Table 4.2.3: Statistical Analysis of MEMS Data for All Engines .........................................62

Table 4.3.1: Comparison of Different Engines of the Same Engine Model on the Same Route ..63

Table 5.5.1: Comparison of Measured and Predicted Emissions Values for Lighter Weight when Weight Difference is Considered and Not Considered .........................................................76

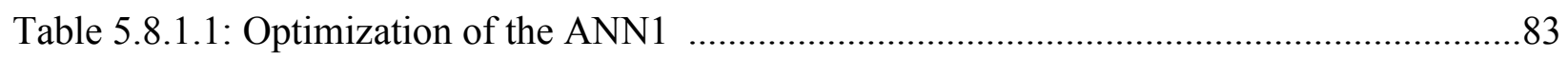

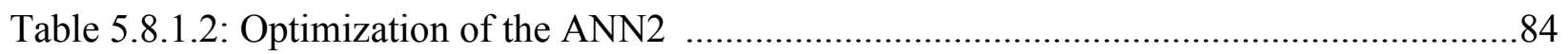

Table 6.1.1: Verification of ANN for Training with, and Predicting Bruceton Mills, WV Routes .93

Table 6.2.1: Verification of ANN for Training with, and Predicting Washington, PA Routes ....99

Table 6.3.1: Verification of ANN for Trained with Washington, PA and Used for Prediction of Bruceton Mills, WV Routes 103

Table 6.3.2: Verification of ANN Trained with Washington, PA Data and Used for Prediction of Bruceton Mills, WV Routes 108

Table 6.4.1: Verification of ANN Trained with Bruceton Mills, WV Data and Used for Prediction of Bruceton Mills, WV Routes 111 
Table 6.4.2: Verification of ANN Trained With Washington, PA Data and Used for Prediction of Washington, PA Routes

Table 6.5.1: Predicted Values, Measured Values, and EPA Regulations for ANN Trained with Bruceton2Sab 1 and Washington, PA1 Engine Dynamometer Data

Table 6.5.2: Comparison of Predicted Values and Measured Values to EPA Regulations for ANN Trained with Bruceton2Sab 1 and Washington, PA1 Engine Dynamometer Data 120

Table 6.5.3: Predicted Values, Measured Values, and EPA Regulations for ANN Trained with Washington, PA2 1 and Bruceton Mills, WV Engine Dynamometer Data

Table 6.5.4: Comparison of Predicted Values and Measured Values to EPA Regulations for ANN Trained with Washington, PA2 1 and Bruceton Engine Dynamometer Data

Table 6.6.1: Predicted and Measured Values for $\mathrm{HC}$ and $\mathrm{CO}$ for ANN Trained with Washington, PA2 1 and Sabraton, WV to Bruceton Mills, WV Engine Dynamometer Data .123

Table 6.6.2: Comparison of Predicted Values of $\mathrm{CO}$ and $\mathrm{HC}$ to Measured Values from Engine Dynamometer Data for ANN Trained with Washington, PA2 1 and Bruceton Engine Dynamometer Data .124

Table 6.6.3: Predicted and Measured Values for $\mathrm{HC}$ and $\mathrm{CO}$ for ANN Trained with Bruceton2Sab 1 and Washington, PA1 Engine Dynamometer Data .124

Table 6.6.4: Comparison of Predicted Values of $\mathrm{CO}$ and $\mathrm{HC}$ to Measured Values from Engine Dynamometer Data for ANN Trained with Bruceton2Sab 1 and Washington, PA1 Engine Dynamometer Data .125

Table 6.7.1: Comparison of Predicted and Measured Values of $\mathrm{NOx}$ and $\mathrm{CO}_{2}$ for the ANN Trained with In-Use Data .126

Table 6.7.2: Comparison of Predicted and Measured Values of $\mathrm{NOx}$ and $\mathrm{CO}_{2}$ for the ANN Trained with In-Use Data .133

Table 6.8.1: Comparison of Measured and Predicted Emissions when the ANN was Trained with a $400 \mathrm{hp}$ Engine and Used to Predict a $350 \mathrm{hp}$ Engine .138

Table 6.8.2: Comparison of Measured and Predicted Emissions when the ANN was Trained with a $400 \mathrm{hp}$ Engine and Used to Predict a $350 \mathrm{hp}$ Engine 140

Table 6.9.1: Confidence Interval Summary for Manufacturer A 400 hp Engine 143

Table 6.9.2: Confidence Interval Summary for Manufacturer A 350 hp Engine, Manufacturer B Engine, and Manufacturer C Engine .143 
Table 7.1.1: Emissions Predicted without Road Grade as an Input 


\section{Nomenclature}

\begin{tabular}{ll} 
ANN & Artificial Neural Networks \\
bhp & Brake Horsepower \\
CAA & Clean Air Act \\
CBD & California Air Resource Board \\
CO & Central Business District \\
CO $_{2}$ & Carbon Monoxide \\
COV & Carbon Dioxide \\
EGR & Coefficient of Variance \\
EPA & Exhaust Gas Recirculation \\
FOREMORE & Environmental Protection Agency \\
FTP & Forecast of Emissions from Motor Vehicles \\
g & Federal Test Procedure \\
GA & Grams \\
HC & Genetic Algorithm \\
hp & Hydrocarbons \\
IBIS & Horsepower \\
MEMS & Integrated Bus Information System \\
MOVES & Mobile Emissions Measurement System \\
NOx & Motor Vehicle Emissions Simulator \\
ODEE & Oxides of Nitrogen \\
PM & On-Road Diesel Emissions Characterization \\
ppm & Particulate Matter \\
PSAT & Parts per Million \\
SCF & Powertrain System Analysis Toolkit \\
VOCs & Speed Correction Factor \\
& Volatile Organic Compounds \\
\hline
\end{tabular}




\section{Introduction, Objectives, and Contributions}

\subsection{Introduction}

Since the early 1960's regulations have been implemented concerning exhaust emissions from heavy duty trucks and buses, specifically with diesel engines. The regulations are concerned with limiting the quantities of gaseous and particulate emissions from heavy duty diesel vehicles. In 1973, the first regulations were implemented to limit the quantity of oxides of nitrogen (NOx), carbon monoxide (CO), and hydrocarbons (HC), it was not until 1988 that a regulation included limits on the amount of particulate matter (PM) emitted [1]. As researchers became more aware of the health and environmental effects of diesel engine emissions, more strict emission regulations have been implemented.

Carbon monoxide is an odorless, invisible gas that is a result of incomplete combustion. Carbon monoxide can cause nausea, headache, and in high enough doses, death. Hydrocarbons are the result of unburned or partially burned fuel. Since diesel fuel is a compound consisting mainly of hydrogen and carbon, the unburned carbon and hydrogen atoms are free to form hydrocarbons [2]. PM is often visible to the human eye as smoke; however it is more frequently present as fine particles which are not visible. It is the small particles that have the greatest negative impact on individuals who are exposed to diesel engine exhaust. Particulate matter is not only an esthetic nuisance, it is also responsible for health issues. The American Lung Association and California Air Resource Board (CARB) have stated that 15,000 premature deaths annually can be attributed to particulate matter produced from diesel engines [3]. Also it has been shown that children who are exposed to PM have reduced lung function and a higher occurrence of asthma related issues. The fine particles pass through the membranes of human lungs, resulting in them becoming imbedded in deep pockets of the lungs, which can hinder biological processes [3]. Other health issues that have been attributed to PM exposure include coughing, decreased lung function, weakening of the heart, breathing difficulty, and aggravated pre-existing conditions. Individuals who already suffer from asthma, bronchitis, and emphysema are more susceptible to experience the effects of PM [2]. NOx is the term given to combinations of oxygen and nitrogen atoms, and is of concern to the environment due to its contribution to ground-level ozone when it reacts with hydrocarbons and sunlight. Ozone is the main constituent in smog, which results in limited visibility and negative health effects. Increased permeability of lung tissue is one of the known 
effects of ozone exposure. When lung tissue is more permeable, toxins and bacteria are more likely to enter and remain in the lungs [4]. Individuals who are already plagued by allergies become more susceptible to allergens due to this increased permeability [5]. Aside from the above mentioned health effects, diesel engine emissions also contribute to the occurrence of acid rain and the greenhouse gas inventory. In order to understand the breadth of the negative impacts of diesel engine emissions, the quantity of these particular constituents attributed to diesel engine emissions must be determined.

The EPA has estimated that heavy-duty diesel vehicles contribute sixty percent of the on-road particulate matter emissions and twenty-seven percent of the on-road NOx emissions [6]. Other sources have estimated that heavy duty diesel engines are responsible for between thirty and sixty percent of on-road NOx emissions [7]. These high percentages are particularly important because heavy-duty diesel vehicles only make up two percent of the on-road traffic [6]. The uncertainty in the quantity of emissions contributed by heavy duty diesel engines is due to a limited understanding of the effects of test cycle, deterioration, and engine programming on inuse emission rates [9]. In order to reduce the health and environmental impact of diesel engine emissions, the allowable levels of emission constituents have been reduced.

The current, more stringent emission regulations only apply to newly manufactured engines. Engines that are already in service are not required to conform to new emission standards. Since older engines are still in use, it is necessary to have a means by which to predict their emissions in order to accurately arrive at an emission inventory value. According to the EPA, engines currently in operation, which are not required to meet new standards, may still be in operation for the next 25 to 30 years [4]. Table 1 displays the emission regulations from 1988 to the present. Between 1988 and 1998 the allowable emission values were regulated differently for trucks and buses, since 2002 both trucks and buses must meet the same standards. It is shown that over the years acceptable emissions levels have decreased, by one to two orders of magnitude. 
Table 1.1.1: Emissions Regulations [10]

\begin{tabular}{|c|c|c|c|c|}
\hline \multicolumn{5}{|c|}{ Trucks } \\
\hline & \multicolumn{4}{|c|}{ Emission Constituent (g/bhp-hr) } \\
\hline Year & $H C$ & $\mathrm{CO}$ & NOx & $P M$ \\
\hline 1988 & 1.3 & 15.5 & 10.7 & 0.6 \\
\hline 1990 & 1.3 & 15.5 & 6 & 0.6 \\
\hline 1991 & 1.3 & 15.5 & 5 & 0.25 \\
\hline 1994 & 1.3 & 15.5 & 5 & 0.1 \\
\hline 1998 & 1.3 & 15.5 & 4 & 0.1 \\
\hline \multicolumn{5}{|c|}{ Urban Buses } \\
\hline & \multicolumn{4}{|c|}{ Emission Constituent (g/bhp-hr) } \\
\hline Year & $H C$ & $\mathrm{CO}$ & NOx & PM \\
\hline 1991 & 1.3 & 15.5 & 5 & 0.25 \\
\hline 1993 & 1.3 & 15.5 & 5 & 0.1 \\
\hline 1994 & 1.3 & 15.5 & 5 & 0.07 \\
\hline 1996 & 1.3 & 15.5 & 5 & 0.05 \\
\hline 1998 & 1.3 & 15.5 & 4 & 0.05 \\
\hline \multicolumn{5}{|c|}{ Both Trucks and Buses } \\
\hline & \multicolumn{4}{|c|}{ Emission Constituent (g/bhp-hr) } \\
\hline Year & NMHC & $\mathrm{CO}$ & NMHC+NOx & $P M$ \\
\hline 2002 Option 1 & NA & 15.5 & 2.4 & 0.1 \\
\hline \multirow[t]{2}{*}{2002 Option 2} & 0.5 & 15.5 & 2.5 & 0.1 \\
\hline & \multicolumn{4}{|c|}{ Emission Constituent (g/bhp-hr) } \\
\hline Year & NMHC & $\mathrm{CO}$ & NOx & $P M$ \\
\hline $2007 / 2010$ & 0.14 & 15.5 & 0.2 & 0.01 \\
\hline
\end{tabular}

Currently, multiple methods exist for modeling and predicting emissions data. The simplest emissions estimation method employs look-up tables of previously obtained emissions data. Two of the most commonly used methods rely on continuous axle power, speed and torque data. The method of using vehicle speed to predict exhaust emissions employs an average schedule speed and what are known as speed correction factors (SCFs). It is common practice for the value of the SCF to be one at the average schedule speed. The SCFs are determined by examining the relationship between speed and the emission constituent of concern. Once the correction factors are determined, emissions from a test schedule with a different average speed can be predicted based on the emissions from the modeled test schedule. It has been shown that this modeling method does not produce consistently accurate results. Error is introduced when 
two test schedules have similar average speeds, but different variations in the speed levels during the schedule [11].

The method of using vehicle power to predict exhaust emissions requires continuous emissions data and axle power data. This method relies on a curve fit between the instantaneous emissions and power at the axle. The function associated with the curve fit is then used to predict emissions based on the axle power of other test schedules. Using axle power to predict NOx emissions does not provide accurate results, and should only be used in cases where rough estimates are sufficient [11]. Ramamurthy et al. also researched predicting diesel engine emissions by correlating axle power to emissions. $\mathrm{CO}, \mathrm{NOx}$, and $\mathrm{CO}_{2}$ were plotted as functions of axle power, and curves were fitted to the data. Once correlations were formed, the functions were used to predict emissions data associated with various test cycles. It was determined by these researchers that axle power is a sufficient predictor of $\mathrm{CO}_{2}$ and $\mathrm{NOx}$ emissions; however, the prediction of $\mathrm{CO}$ emissions based on axle power was inaccurate. Discrepancies between the predicted and experimentally obtained emission values can result if the cycle used to establish the correlation between the emission constituent and axle power does not span the full range of power for the specific vehicle being examined [12].

Joumard et al. addressed the possible errors and issues encountered when modeling in-use emissions. Many models rely on average speed to predict emissions over a driving cycle, however this can introduce error in predicted emissions because it has been shown that significant changes in speed can impact instantaneous emissions by two to three times. Errors are also incorporated into emissions modeling via measurement errors and modeling errors. When data is recorded, it is important to be sure measurement and recording instrumentation is functioning as expected. It is also important to document environmental conditions, due to their impact on emissions levels. Modeling errors may occur if inadequate parameters are employed by the model, resulting in the model not accurately predicting emissions from the applied database. The researchers recommend in order to accurately predict emissions, both instantaneous operating conditions and an operating condition history should be considered [13]. 


\subsection{Objectives}

The global objective of this dissertation work was to develop a model that can accurately predict in-use heavy duty diesel engine emissions by employing engine dynamometer data available through the previous work of the West Virginia University, CAFEE. The major objectives that led to the accomplishment of the global objective are listed below.

- Identify most applicable and effective modeling method.

- Develop an emissions model that can predict in-use emissions from engine dynamometer data.

- Verify the model by comparing results with experimental data and EPA regulations.

- Present a final working model to accurately predict in-use emissions

\subsection{Technical Approach}

The work required to achieve the above mentioned objectives was divided into the tasks explained below.

\section{A. Literature Review}

Conducted a review of literature pertaining to heavy duty diesel engine emissions research, standards, modeling, and prediction methods.

\section{B. Data Survey}

Located and determined availability of engine dynamometer data for heavy duty diesel engines employed in trucks and buses. Determined the availability of in-use data for model verification.

\section{Model Development}

Determined the optimum modeling method and developed a model that accurately predicts in-use heavy duty diesel emissions from engine dynamometer data, taking into account grade effects.

- Problem Definition and Formulation

○ Defined desired output and determined required inputs

- System Design

○ Determined structure for ANN

○ Determined most applicable learning algorithm 
○ Collected and pre-processed data

- System Realization

- Trained ANN with specified data

- Evaluated initial outputs and errors

D. Model Optimization

Varied model characteristics in order to reach the most accurate predictions of in-use emissions data.

E. Model Verification

Compared model output results to actual in-use data in order to determine the model's accuracy. 


\section{Literature Review}

\subsection{Current Models}

Currently, few widely used computer models exist for predicting emission inventories and in-use emissions from heavy duty diesel vehicles. The Environmental Protection Agency (EPA) and the California Air Resource Board (CARB) are the agencies most concerned with emissions modeling in the United States. The most commonly used models are EPA's MOBILE, CARB's EMFAC, and EPA's MOVES. The EPA also employs particulate matter estimation models known as PART5 and PART6. CARB's prediction methods are based on engine certification data and a limited number of chassis dynamometer tests [14]. The EPA also developed the Mobile Emission Assessment System for Urban and Regional Evaluation, known as MEASURE. MEASURE was developed in the late 1990s, in order to predict the effect of suburban sprawl and commuting to metropolitan areas on emissions. The purpose of the program was to aid transportation designers in analyzing the impacts of actions such as signal timing and adding lanes [15]. The most recently released emission model developed by the EPA is MOVES2010, which replaced MOBILE6.2.

\subsubsection{MOBILE}

The EPA developed the first version of its MOBILE software in the 1970s, it was denoted as MOBILE1. Since its inception, the MOBILE model has been updated with releases MOBILE2, MOBILE3, MOBILE4, MOBILE 4.1, MOBILE5, MOBILE5a, MOBILE5b, MOBILE6 and MOBILE6.2. The Clean Air Act (CAA) requires the EPA to update the emissions estimation programs, and release currently applicable versions. With each release, the models have included more in-use data, have been updated to be compatible with new technologies, and have accounted for more factors when estimating engine emissions. MOBILE1 was released in 1978, and used age and mileage of vehicles in order to arrive at estimated emission values. MOBILE2 and MOBILE3, released in 1981 and 1984, respectively, took into account newer vehicle technologies including catalytic converters and the effect of tampering. New in-use data and more user control options were incorporated into MOBILE4, released in 1989. MOBILE4.1 was released in 1991 and incorporated the effects of operation and maintenance programs, as well as the impact of the newest emissions regulations. Due to state implemented test programs, a larger data set was available and new equations were derived to predict emissions for the 1993 release 
of MOBILE5 and MOBILE5a. The impacts of oxygenated and reformulated fuels on emissions were also examined in the MOBILE5 series. In 1996 MOBILE5b was released which was updated to include the newest emission regulations, and the ability to estimate idle emission factors was included. The release of MOBILE6.0 occurred in 2002, and the new program was equipped with more in-use deterioration data, and updated for newer engine and fuel technologies. The ability to model air toxins such as benzene and formaldehyde and improved carbon monoxide prediction values were features of the MOBILE6.2 program, released in 2004 [16].

\subsubsection{Powertrain System Analysis Toolkit}

The United States Department of Energy has contributed to the development of modeling software based in MATLAB, which is called Powertrain System Analysis Toolkit (PSAT). PSAT is a vehicle simulation toolkit that estimates vehicle performance from a calculated component torque response to realistic commands. This software is widely used in the automotive industry for vehicle simulations and modeling. Inputs such as engine throttle, clutch displacement, and transmissions gear number are employed in the software. PSAT is capable of modeling a variety of vehicle technologies including conventional, electric, fuel cell, and hybrid. PSAT is capable of accurately predicting emissions for heavy duty diesel engines over various cycles, however it requires extensive input information, some of which may not be known for vehicles that need to be modeled. For example, a vehicle may be equipped with a specific engine, but information about the transmission could be unavailable [55].

\subsubsection{MOVES}

As mentioned above, the EPA plans to replace the MOBILE series of emission prediction programs with a newly developed program called Motor Vehicle Emissions Simulator, also known as MOVES. MOVES2010 includes more in-use data than the previous MOBILE series and is also able to predict the emission levels of more Volatile Organic Compounds (VOCs). Initial comparisons of MOVES2010 to MOBILE6.2 show that MOVES2010 predicts lower

values for emitted VOCs in urban areas, higher values for NOx emitted, and higher quantities of particulate matter emitted [17]. These differences in the two models show that there is still room for improvement and uncertainty in the emissions predictions modeling realm. 


\subsubsection{IBIS}

Wayne et al. have developed a modeling tool to predict the emissions associated with fleets of transit vehicles, this model titled, Integrated Bus Information System (IBIS), allows a user to determine the emissions associated with a particular fleet of vehicles. The use must enter information about the vehicles in the fleet being examined such as type of fuel, model year, curb weight, powertrain type, engine rated power, aftertreatment equipment, transmission type, heating and air-conditioning capacity, and displacement. For the route being examined, information such as the average speed, percentage idle, number of stops per mile, standard deviation of speed, and the kinetic intensity must be known. The model predicts the fuel economy, and emissions of $\mathrm{NO}_{\mathrm{x}}, \mathrm{CO}_{2}, \mathrm{CO}, \mathrm{PM}$, and $\mathrm{HC}$. The data employed to develop this model was obtained from chassis dynamometer testing, and then polynomial fits and linear regressions were applied to the data. In cases where data were not available for a particular scenario, genetic algorithms were employed to predict the emissions for that situation. The purpose of this modeling tool is to allow fleet owners to compare and contrast the emissions associated with fleets of different characteristics, in order aid in planning and procurement decisions [66].

\subsubsection{Accuracy of MOBILE Models}

It has been determined through prior work that actual measured emissions data from heavy-duty diesel vehicles differ from the results that are predicted through the use of models such as MOBILE5 and PART5. The measured emissions values also differ from the data that has resulted from engine certification tests. One of the reasons it is important to accurately estimate the emissions from vehicles is to determine if the regulations that have been put into effect are making a difference. Also, in order to examine, monitor, and plan air quality, pollutant inventories are developed, which are based on the estimated emissions. In-use emissions are functions of driving cycle, inertial weight, and drive trains. Plots of NOx versus power were constructed and examined as part of the research of Yanowitz et al., and it was determined that it is possible to employ chassis testing and a transmission model to determined if in-use NOx emissions agree with the engine certification results. This work also concluded that emitted carbon monoxide $(\mathrm{CO})$ values on a brake-specific mass basis agree between chassis and engine dynamometer tests. Particulate matter emissions were determined to be underestimated by 
engine certification tests, when compared to chassis data on brake-specific mass basis [18]. Singh et al. also noted that current emissions prediction programs such as those discussed previously are only capable of predicting emission inventories at a county-size scale. It was the recommendation of the researchers that a program be developed that is able to predict PM emissions on a smaller scale, therefore the impact on humans and the environment at specific sites can be examined [19].

Studies have shown that PART5, the EPA's PM emission factor model, estimates PM production to be much less than what is actually emitted during in-use conditions. The PART5 program was revised to better estimate PM emissions, by examining the data produced by four test vehicles, two trucks and two buses. In order to make revisions to the PART5 model, the assumptions that the model employs were examined. It was shown that the PART5 model assumes that PM emissions from a vehicle will not ever exceed the level at which it was certified. This assumption introduces error by assuming that the technologies used to meet the new, more stringent emission standards will not deteriorate with time or fail. As the technologies get more complicated, resulting in a greater reduction of emissions, there is also the fact that if they fail to operate optimally, greater emissions than expected will result. A study by Whitney determined that the PART5 estimated PM emissions were three to 11 times lower than those actually emitted by light-duty vehicles, most specifically seen in vehicles with an excess of 100,000 miles [20].

It is suspected by researchers that the portion of NOx in emissions inventories attributed to heavy-duty diesel vehicles is under representing the actual contributions of these vehicles. The under-estimation is in part due to the fact that many emission inventories are estimated using data acquired during engine certification testing. One emission prediction model, MOBILE, employs engine certification data and has been shown to predict less NOx emissions than what tunnel and on-road testing have shown. One reason for this discrepancy is that engine producers were programming the engines to operate differently when they were noticeably running the FTP cycle for certification. When these engines were put into vehicles, they produced more emissions than they did during the certification testing. Since the MOBILE model and other emissions prediction models employ engine certification data, some of their predictions have been based on unrealistic data that was acquired during certification testing of these engines. 
Engine manufacturers have since signed consent decrees, in which the manufacturers agreed to retrofit such engines during maintenance, pay fines, and comply to 2004 emissions standards earlier than was originally expected. As part of the consent decrees it was determined that a program would be developed to correlate in-use testing with engine certification data. A division of the EPA developed the On-Road Diesel Emissions Characterization (ODEC) facility, which has the goal of compiling in-use emissions data for a variety of road conditions and vehicles [22].

\subsubsection{Accuracy of EMFAC Model}

Shah et al. compared the measured NOx emissions associated with various driving schedules to those that are estimated by EMFAC. The emissions were measured for eleven vehicles employing engines ranging from 1996 to 2000. It was determined that the EMFAC predictions underestimated the quantity of NOx emitted by five to fifty-seven percent, based on the test cycle being examined [21].

\subsubsection{Accuracy of Emission Factor Models}

McCormick et al. researched comparing data obtained from an engine dynamometer to data obtained from a chassis dynamometer, and evaluated the correlation. For this research two buses and a truck were tested on a chassis dynamometer and then the engines were removed from the vehicles and tested on engine dynamometers. The two transit buses were equipped with 1993 DDC Series 50 engines, while a truck was powered by a Navistar DTA-466 engine. The chassis dynamometer tests employed the CBD and HDT cycles. When the emissions data from the two cycles were compared, it was determined that when compared on a fuel volume basis the HDT and the CBD agreed closely, but when compared on a distance basis a significant disagreement in data was seen. This can be explained due to the effect of inertial weight on all emissions constituents when compared on a distance basis, but when the emissions values are compared on a volume basis, only PM is effected by the inertial weight. Emissions prediction factors in work per distance were then estimated by using the data obtained from these tests. These factors were used to convert engine dynamometer data to distance specific values and then these values were used to estimate emissions inventories. The factors that were determined from this research were then compared to the factors that are currently employed by the EPA in inventory estimation 
methods, and it was found that the factors were substantially different. Accurate estimations of pollution inventories are necessary for air quality planning and determining the effectiveness of regulations. The discrepancy between the EPA estimated emissions factors and the ones determined in this work could be due to the fact that the EPA based its emission factors on the data acquired from engine certification testing. During engine certification testing, new engines are tested on engine dynamometers. In order to estimate the emissions produced by the engine over its lifetime (435,000 miles) a deterioration factor is employed. Engines are still in service that have accrued more than 435,000 miles, therefore assuming that engines are no longer operating after their lifetime is an error inducing assumption. Also, it has not been proven that the EPA's deterioration factor contributes to accurately predicting in-use emissions for all types of engines over their lifetimes. The current EPA factors are based on a comparison of engine and chassis dynamometer testing for a transit bus and three trucks. The emission factor is a ratio of the chassis dynamometer results to the engine dynamometer results [8]. Using engine certification data may also skew data in cases where the engines being tested are equipped with defeat devices. It is unclear if the deterioration factor accounts for lack of maintenance of turbochargers and fuel injectors, which leads to increased PM, HC, and CO emissions. Also it should be noted that an increase in NOx emissions can be attributed to improperly timed fuel injection or poor air cooler performance [23]. The above mentioned deterioration and maintenance issues are not measured during engine certification data and may not be properly accounted for in the deterioration factors used by the EPA, therefore further examination is needed.

Research has resulted in the development of a table of emissions factors that aid in the prediction of in-use emissions. The emission factors are based on instantaneous engine power. The model predicts emissions by employing a series of matrices for the exhaust constituents including NOx, $\mathrm{CO}, \mathrm{CO}_{2}$, and $\mathrm{HC}$, using the inputs of speed and acceleration. The PM emissions were estimated by using a ratio of the known PM emission values over a speed and acceleration range, based on CO emissions. Data acquired via testing by the WVU Mobile laboratory were used to compile the matrices employed by the model. Multiple methods exist to estimate emission inventories, some include using engine certification data, data acquired by chassis dynamometer testing, and ratios of NOx and $\mathrm{CO}_{2}$. MOBILE5 and MOBILE6, the EPA's emission prediction models, and 
EMFAC, CARB's model, are based on data used for engine certification. The FTP cycle does not adequately represent present day driving conditions such as stop-and-go city and freeway conditions, and therefore fails to accurately model the emissions of current vehicles. Using the engine certification data to model engine emissions also does not account for the deterioration, maintenance, and any alterations experienced by the engines. When chassis dynamometer data is used to predict emissions, inaccuracies result from that fact that specific cycles are used to analyze the emissions, and none of the cycles have the ability to model the multitude of driving conditions that a given vehicles will experience. Emission factors based on power allow the emissions to be predicted for a cycle other than the cycle which was used to acquire the data. Issues arise when using this method, because for it to be accurate, time alignment must occur that relates the instantaneous rear-axle power and the emissions. It is possible to measure the instantaneous power easily; however there is a delay between when the power event occurs and when the exhaust gas reaches the measuring instruments. For NOx and $\mathrm{CO}_{2}$ this prediction method has been shown to be successful, however results have not been as promising for the prediction of $\mathrm{CO}$ and $\mathrm{HC}$. Another downfall of predicting emissions based on rear-axle power is that the effect of "off-cycle" injection timing cannot be incorporated. In some cases speed and acceleration are used to predict emissions rather than instantaneous power. The speed is employed to determine losses due to road-load, and the acceleration coupled with the speed can predict the instantaneous inertial power demand. When a vehicle experiences a change in road grade, the effect on emissions is not well documented due to the lack of grade simulation in chassis dynamometer testing. The uncertainty in emissions associated with road-grade changes induces problems into the method of predicting emissions based on speed and acceleration data. When climbing an incline acceleration is low, however significant rear-axle power is required and when travelling in a descending direction acceleration can be high, while limited power is required. This results in the model over estimating emissions for descending terrains, and under estimating emissions when vehicles ascend hills. Along with speed and acceleration, weight also plays a key role in emissions prediction. It is important to account for the weight of a vehicle because the higher the load, generally the higher the emissions for a given speed and acceleration. When examining buses in particular this is a concern since the load of the bus can change with each stop, in the case of tractor-trailers, the load is typically constant over the length of a trip [24]. 


\subsubsection{Emissions Inventory Estimation}

It has been shown that emissions inventory estimation techniques do not account for every factor that impacts emission, and as shown in the reviewed literature, are not as accurate predictors of diesel engine emissions as they could be. Research has shown that emissions inventory estimations are based on data obtained from FTP tests, when engines are new and undergoing certification. In order to test older engines on engine dynamometers the engines must be removed from the vehicle, which requires the vehicle to be out of service during testing, and then time and money must be expended to reinstall the engine into the vehicle. The time involved and financial expense of dynamometer testing is the main reason that data for the emission inventory is limited. Concerns have also been raised, stating that the FTP is over 25 years old, and has not been updated to accurately assess the engines that are equipped with newer technologies. It is thought that data acquired by chassis dynamometer testing would better represent in-use emissions, when compared to the FTP data [25].

Data were obtained by the WVU Mobile Laboratories for emissions from buses and heavy duty trucks. Data for buses were determined by employing the Central Business District (CBD) cycle, while heavy duty trucks were examined with the truck CBD and the WVU 5-peak test cycle. Throughout the duration of the chassis dynamometer tests, the drag on the vehicle and rolling friction of tires were incorporated by taking into account the air density, the frontal area of the vehicle, the drag coefficient, and the friction coefficient. It was determined that the NOx measurements from the chassis tests were within the range of certification provided by FTP data, however the ratio of NOx to $\mathrm{CO}_{2}$ was found to be widely variable, which supports the opinion that the FTP cycle is not always accurate in estimating in-use emissions [25].

\subsubsection{Modal Modeling Methods}

Barth et al. believe that modeling emissions using a modal method will prove to be more accurate than the current methods used by the programs available through the EPA and CARB. Currently the modeling methods assume that it is accurate to use emissions measured from a specific driving cycle in cooperation with a speed correction factor to predict in use vehicle emissions. The authors of this work have shown the need for a model that considers events such as acceleration/deceleration, idle, and steady-state cruise. Currently emissions modeling 
techniques do not account for realistic driving conditions; most models rely on data derived from the FTP cycle. The FTP cycle mimics behavior associated with driving in urban conditions from over twenty-five years ago. Since the development of the FTP cycle in-use urban operations have changed, making it an antiquated tool for predicting current day in-use behavior. The commonly used emission prediction models also rely on average speed to determine speed correction and emission factors. Error is introduced with this method because only the average speed is considered, and not acceleration, deceleration, and idle events. Two driving cycles can share an average speed, and produce dissimilar emissions based on differences in modal operations. It has been shown that a greater quantity of $\mathrm{CO}$ can be produced in a single acceleration event than is produced in an entire four mile trip [47]. The current models also do not consider road grade when predicting in-use emissions, the impact of which will be discussed in a following portion of this document [26].

Jost et al. have developed a multi-layer diesel emissions modeling technique that employs steady-state engine maps, driving curves, and vehicle data to obtain emission factors. It has been shown previously that emissions are a function of engine power, and that relationship is employed in this model. In this work the instantaneous engine power is separated into two segments, steady-state and transient. The power delegated to steady-state applications is consumed to overcome the resistance experienced due to wind, rolling friction, and road grade. Overcoming inertia associated with the vehicle mass during acceleration is defined as the transient consumer of power. The steady-state engine maps were used to predict emissions associated with the power applied to steady-state parameters, and driving curves and transient emissions data were used to predict emissions associated with the power consumed as a result of transient variables. The model varies the steady-state power/transient power ratio based on driving patterns. To predict the emissions associated with a specific vehicle under specific conditions the sum of the results from the steady-state portion and the transient portion is determined. It has been shown that this modeling tactic produces an estimate for NOx, HC, and $\mathrm{CO}$ emissions. $\mathrm{CO}_{2}$ emissions are predicted more accurately than $\mathrm{NOx}, \mathrm{HC}$, and $\mathrm{CO}$ using this method [27]. 


\subsection{Influencing Factors}

\subsubsection{Deterioration Factor}

It has been determined that system deterioration has an impact on emission values in heavy duty diesel engines. Deterioration can be attributed to multiple factors associated with both manufacturer defects and malfunctions. Common malfunctions that impact emissions values include worn turbochargers, fuel injector malfunctions, smoke limiting device failures, mechanical failure of the engine, electronic control failures, and excess oil consumption. Other factors that are classified as deterioration can be attributed to lack of maintenance or human involvement. Neglected maintenance issues such as clogged air filters and clogged intercoolers can affect emission values. Human interference can also attribute to altered emissions, such as tampering with electronic controllers, equipping engines with improper turbochargers, and altering timing [28].

Deterioration of vehicle engine and equipment affect the emissions, therefore a method of predicting a deterioration factor was examined. After examining a sampling of vehicles, it was noticed that newer technology vehicles have a tendency to exceed their certification PM emission levels in a shorter period of time than older technology vehicles. It was concluded that the revised version of PART5 predicted higher PM emissions over the life of a vehicle, then the unedited PART5 model. The authors recommended that future work be performed along the same lines with a larger data set [20].

The effects of system deterioration on in-use diesel engine PM emissions have been examined by Yanowitz et al. In this study twenty-one vehicles were evaluated and it was concluded that a correlation between odometer mileage and PM emissions exists. The same study was conducted with chassis dynamometer testing, and a correlation between the PM emissions and odometer reading could not be established. The researchers attributed this lack of correlation to difference in measuring methods and test conditions at the different chassis dynamometer facilities where the data were acquired [30].

The effects of deterioration on other emission constituents have also been examined. It has been determined that any deterioration that reduces the efficiency of diesel engine combustion, 
increases the quantities of $\mathrm{HC}$ and $\mathrm{CO}$ emitted from the engine. A manner in which to account for deterioration is required in order to obtain an accurate emission inventory [30]. Zachariadis et al. examined FOREMORE (Forecast of Emissions from Motor Vehicles), a software program that has been developed to estimate future levels of engine emissions. It was determined that the examined software did not accurately account for deterioration of engine systems and the associated impact on emission values. FOREMORE relied on a linear function to estimate the effects of deterioration; the function was based on in-use emission values associated with specific mileages, not vehicle ages. Three specific aspects of deterioration should be considered when examining the impact of vehicle emissions, average age of vehicle fleet, decrease of average specific mileage with vehicle age, and the deterioration of emissions control systems with age. The FOREMORE software produced unrealistic results by assuming that vehicle miles travelled was independent of vehicle age, and that the emission factors are independent of age. It should be noted that age of a vehicle and the associated technological alterations have a significant impact on engine emissions [31].

McDonald has researched developing a deterioration factor by comparing the average emissions of vehicles with 50,000 miles to the average emissions of vehicles with 4,000 miles, and employing a linear regression technique. $\mathrm{NO}_{\mathrm{x}}$ and $\mathrm{CO}$ were examined, and it was determined that the correlation between mileage, NOx, and $\mathrm{CO}$ was not accurately represented linearly during the examined mileage range [12]. Ntziachristos et al., however, feel that deterioration can be accurately accounted for by employing a linear function based on mileage, up to the point where 74,565 miles is reached [33].

\subsubsection{Road Grade}

Along with deterioration, road grade has an impact on in-use exhaust emissions. The current emission prediction software released by the EPA does not include road grade in its emissions prediction analysis. The EPA has stated that not enough data and time were available to address the road grade factor in MOVES, however they acknowledge that road grade, and the coupling of road grade and deterioration may have a significant impact on actual in-use emission values. Road grade has been shown to have a measurable effect on $\mathrm{NOx}, \mathrm{CO}$, and $\mathrm{CO}_{2}$ emissions, most specifically when the road grade is greater than two percent [34]. 
Khan examined the impact of road grade on emissions and fuel consumption of buses that employed a diesel engine. A model was developed that predicted the fuel consumption as a function of speed, weight, and road grade. This model simply used a sinusoidal input to model road grade [35]. The influence of road grade on emissions factors was examined and discussed by Antonacci et al. These researchers studied the effect of road grade on emissions in mountain areas near the Alps. It has been shown that when the average road grade is greater than two percent, the increase in emissions experienced by ascending vehicles is not negated by the decrease in emissions experienced by descending vehicles. A case study which examined a transit route between Italy and Austria found that for a route with an average road grade of 3.5 percent, $\mathrm{NO}_{\mathrm{x}}$ emissions were 16 percent higher than they would be if the terrain was flat [29].

\subsection{Defeat Devices and Consent Decrees}

Engines produced by the six leading heavy duty diesel engine manufacturers in the 1990's were determined to be equipped with technology that altered their injection timing during certification testing. This altered injection timing resulted in a reduction in emissions production during certification testing, which meant the engines produced higher levels of certain emissions when they were put into real world driving conditions than they had during engine dynamometer testing with the FTP cycle. Such devices were declared to be defeat devices and were determined to be illegal by the United States government. The United States and each engine manufacturer entered into an agreement known as a Consent Decree. The Consent Decrees required that the engine manufacturers cease the employment of defeat devices, and altered the method by which engines were certified to meet emissions standards [70].

\subsection{Artificial Intelligence Modeling Techniques}

Multiple artificial intelligence modeling methods exist, including expert systems, case based reasoning, bayesian networks, genetic algorithms, fuzzy logic, and neural networks.

\subsubsection{Expert Systems}

Expert systems are generally employed in situations where mathematical algorithms are not applicable. The expert system is programmed with a series of rules based on knowledge from an expert in the applicable field, and then these rules are applied to the input information. The 
output of the expert system is a result of the cause and effect relationship between the programmed rules and the inputted information. A special type of expert system is known as case based reasoning, which relies on the solutions to similar, previously solved problems to arrive at a solution to the current problem.

\subsubsection{Bayesian Networks}

Bayesian networks are graphically based, and incorporate events and probabilities. It is most applicable in situations where one is concerned with the likelihood of an event occurring or the dependency of one event on another. When parameter optimization is the objective of a model, genetic algorithms are applicable.

\subsubsection{Genetic Algorithms}

Genetic algorithms search a defined solution space for a set of data that can serve as a solution to the problem being examined. Each set of data examined is referred to as an individual and the algorithm operates analogous to Charles Darwin's theory of evolution. Only the individuals which meet predefined criteria are permitted to combine with other individuals to create new individuals. As in the evolution of a species, only the most fit survive to produce more individuals. The process continues until a preset number of generations of individuals have been evaluated or a certain individual meets a performance criterion [36]. The basic logic followed in any genetic algorithm is shown below in Table 3-2. 
Table 2.4.3.1: Genetic Algorithm Approach [37]

\begin{tabular}{|c|l|}
\hline \multicolumn{2}{|c|}{ Genetic Algorithm Approach } \\
\hline Step \# & \multicolumn{1}{c|}{ Action } \\
\hline 1 & Initialize population with random numbers within each parameter's range \\
\hline 2 & Computer output values for each population set \\
\hline 3 & Calculate fitness level of each member based on pre-determined standards \\
\hline 4 & For members whose values exceed required value, set fitness value to zero \\
\hline 5 & Select remaining members of the population based on probability selection for crossover \\
\hline 6 & Generate new members, forming next generation of the population \\
\hline 7 & $\begin{array}{l}\text { Re-visit Step } 2 \text { and continue process until desired number of generations is reached or the } \\
\text { stopping criteria is met }\end{array}$ \\
\hline
\end{tabular}

\subsubsection{Artificial Neural Networks}

Artificial neural networks, like genetic algorithms, are modeled after biological phenomena. Artificial neural networks are modeled after the nervous system and the function of the brain. The brain is composed of neurons that interact via connections, each neuron can be linked to up to 200,000 other neurons. The neurons in the human brain are made up of three main components, somas, dendrites, and axons. The dendrites are branchlike extensions that serve as the entry point for information. The soma serves as the main body of the neuron and acts as a gathering point for all of the information that enters via the dendrites. The outputs of the neurons exit through the axon. Neurons have multiple dendrites or input points, but only a single axon or output point. Figure 2.4.4.1 depicts the basic architecture of a human neuron. 


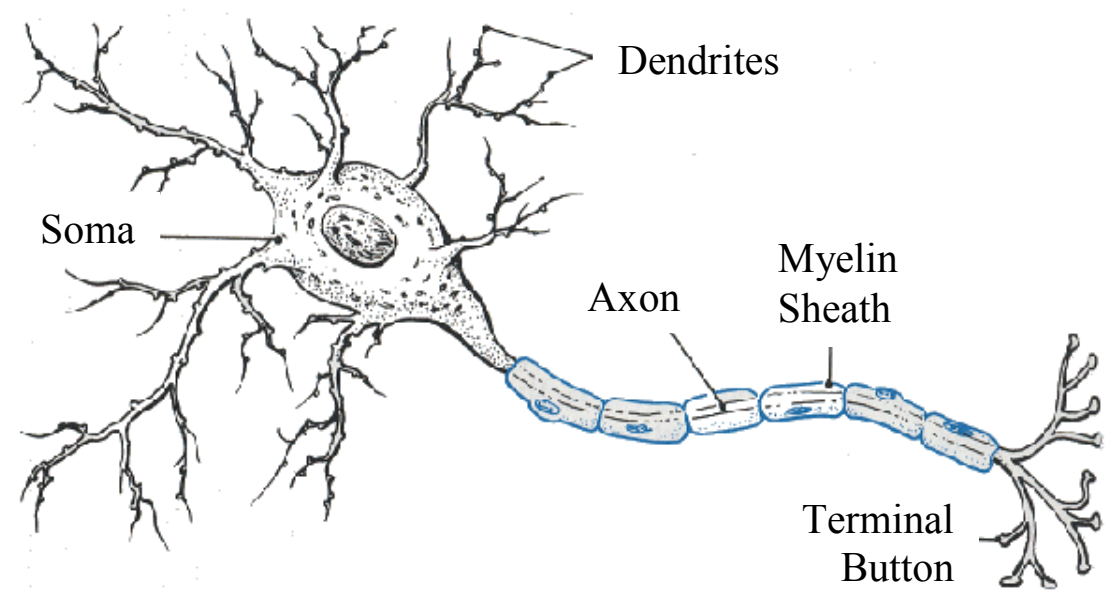

Figure 2.4.4.1: Schematic of Human Neuron [38]

The structure of the brain's neurons lends itself to mathematical modeling based on the multiple inputs, single output operation. Figure 2.4.4.2 below depicts a schematic of the basic artificial neuron, the inputs are represented by the letter " $x$ " and the output is represented by the letter " $y$ ". It is shown that multiple inputs are summed or evaluated together in order to produce a single output. This output is commonly referred to as the activation, and is derived by applying weights to all inputs and using their values in a specified activation function. This function is commonly referred to as the transfer function, and it typically exhibits non-linear behavior for a sub-set of real numbers [39].

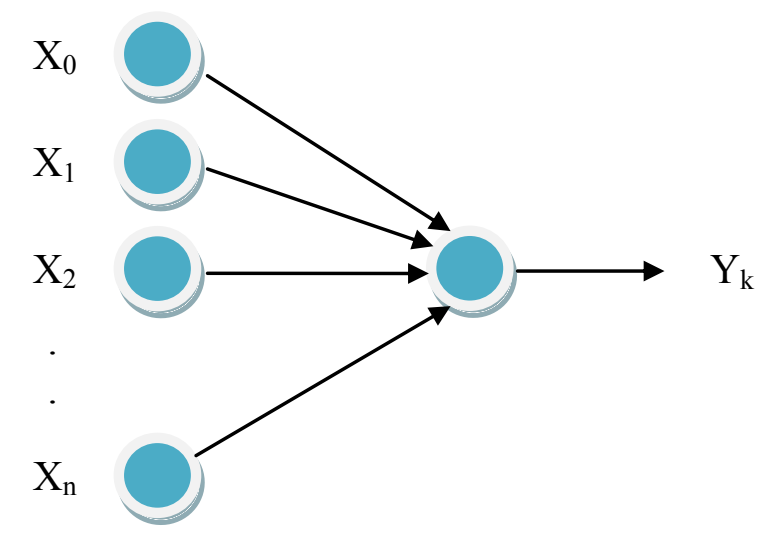

Figure 2.4.4.2: Schematic of Artificial Neuron [39] 
Artificial neurons can be divided into three specific types, input neurons, output neurons, and hidden neurons. Input neurons serve as receptors to acquire information from outside the actual network, while output neurons send information outside of the network or inside the network via feedback paths. Unlike the input and output neurons, hidden neurons can only send or receive information from inside the network. These types of neurons are combined in different manners to make-up the structures of the neural networks. Neural networks always have multiple inputs, but can have differing numbers of outputs, layers, and hidden layers. The networks can also be designed to have information flow in a single direction or multiple directions. Networks that allow information to only flow in one direction are referred to as static or feed-forward networks. If information flows backwards as well as forwards, the network is referred to as a feedback or recurrent network. An example of this would be if a neuron's output was employed as the input by a neuron in a previous layer [40].

One of the defining factors of a neural network is its ability to learn. Learning describes the process by which the weights of internal parameters or connections between nodes are altered. Learning is said to occur when the weights are altered until the target output value is obtained using a specified data set as inputs. Three different learning types exists, supervised or error based, unsupervised or output-based, and reinforcement or performance index-based. Supervised learning requires data sets have input and output data. The weight alterations occur based on the error between the calculated output and the expected output value. The weights are adjusted until the error between the calculated output value and the expected output value reaches a value deemed acceptable. Unsupervised learning does not require outputs as part of the training data. In this method the synaptic weights are adjusted based on the relationship between the neurons on either side of the synapse. The weights are increased based on the activity of the synapse; more active synapses have higher weights, while less active ones receive a decrease in weight. Eventually, the neuron that is active the most remains, while all other neurons become inconsequential due to the low weighting of their adjacent synapses. The performance index learning method does not require pairs of inputs and outputs. Rather than employing the input and output training data directly, a performance index is designed to evaluate the data. The synaptic weights are altered based on the effect they have on the performance index. The weighting is increased if a positive effect on the performance index 
occurs, and a decrease in weight is experienced if a negative effect on the performance index occurs [40].

The artificial neural network model does not require details about the exact construction of the system being modeled; rather it arrives at its outputs based on a learned relationship between input data and controlled and uncontrolled variables. This relationship is learned from data sets that are obtained previously, and employed by the model. The accuracy of a neural network is impacted by the activation or transfer function and the number of hidden neurons. It is important for the program developer to select an appropriate transfer function and number of neurons. Various transfer functions are employed in artificial neural network applications such as Gaussian, Gaussian Complement, Sigmoid Logistic, and Symmetric Logistic. Each of these transfer functions are most applicable to certain types of data. For example, the Gaussian transfer function is most applicable when the important characteristics of the data are not the extreme values, this function transforms the extreme values of the data to low values, and average values in high values, while normalizing the outputs to values between 0 and 1 . If the extreme data points have the most impact on the data characteristics then the Gaussian complement transfer function should be employed. The most commonly used transfer function is the Sigmoid Logistic function, this function transforms the input data to values that range from 0 to 1 . In contrast, the Symmetric Logistic Function transforms input data to values between -1 and 1. Table 2.4.4.1 displays each type of transfer function addressed, and the associated mathematical function [58]. In this table $\mathrm{n}$ represents the input into the neural network. 
Table 2.4.4.1 Commonly Used Transfer Function in ANN [58]

\begin{tabular}{|c|c|}
\hline Transfer Function Type & Mathematical Function \\
\hline Gaussian & $f(n)=\exp \left(-n^{2}\right)$ \\
\hline Gaussian Complement & $f(n)=1-\exp \left(-n^{2}\right)$ \\
\hline Sigmoid Logistic & $f(n)=\frac{1}{1-\exp (-n)}$ \\
\hline Symmetric Logistic & $f(n)=\frac{2}{1-\exp (-n)}-1$ \\
\hline
\end{tabular}

Weighting factors are incorporated into the program, and are altered based on rules which results in an input and output relationship that accomplishes the desired result. It is also necessary that a sufficiently large, yet not exceedingly large range of data is used to train the algorithm. If too small of a training set is employed the algorithm will not be accurate when dealing with data outside the range, if the training set is too large an understanding of realistic conditions may be lost.

\subsubsection{ANN Applied to Emissions Modeling}

Artificial neural networks have been successfully applied to model emissions from natural gas combustion engines. The input variables for this work were charge pressure, charge temperature, start and duration of combustion, and equivalence ratio. The outputs of the neural network were NOx emissions and engine efficiency. It was determined that both the genetic algorithm and the neural network approaches are appropriate to predict engine efficiency and NOx emissions of engines.

Further research has focused solely on predicting NOx emissions by employing an ANN. One work in particular focused on predicting NOx emissions in order to determine the quantity of reductant to add via an emission reduction system. In current NOx reduction techniques, either data acquired by a NOx analyzer or predicted based on an engine map dictate the quantity of reductant to be injected. This work considered replacing the NOx analyzer with an ANN due to 
the reduced financial, maintenance, and space demands of the neural network compared to the current NOx analyzers. The accuracy of the neural network was determined by comparing its outputs to the NOx predicted by an engine map and a linear fit. The researchers designed a supervised, multilayer perceptron neural network, which was tested with varying quantities of nodes. The number of hidden layers was varied between zero and two, and the number of nodes was varied between eight and 40. The inputs were engine speed, both at current time and one time step previous, rack position, both at current time and one through four time steps previous, charge air pressure, at current time, one time step and two time steps previous, and also charge air temperature, at the current time only. After running multiple variations with hidden layers and node numbers, it was determined that the most accurate results were achieved with one hidden layer and between 20 and 40 nodes. With 30 nodes the average absolute error between the measured and calculated NOx emissions was determined to be $5.2 \%$. It was determined that the neural network is a feasible and accurate method to predict NOx emissions from a heavy duty diesel engine [11]. Obodeh et al. also researched the applicability of neural networks to NOx emission prediction for heavy duty diesel engines. The inputs for the neural network were engine speed and load, and the outputs were NOx, power, and specific fuel consumption. It was determined that these inputs were able to accurately predict the desired output, and that ANNs are applicable to predicting NOx emissions [42].

Previously mentioned works required a minimum five inputs to predict emissions by employing an ANN. Arcaklioglu et al. have designed an artificial neural network that predicts exhaust emissions from a diesel engine by employing three inputs. The three required inputs are engine speed, accelerator pedal request, and injection pressure. This research focused on four-cylinder, four-stroke diesel engines, equipped with a turbocharger and indirect injection. The testing included both full and partially loaded scenarios and different injection pressure and throttle positions. The ANN was trained with results for engine speed, injection pressure, and throttle position that were obtained experimentally. The method of back-propagation learning was used, employing a single layer and two hidden layers. During the testing and design of the algorithm, results obtained with differing numbers of neurons and layers were examined. This work showed that an ANN can be used to successfully predict exhaust emissions, with limited inputs, 
from a diesel engine, most specifically when steady results can be produced from the same experiment [43].

The development of a neural network model of a four-cylinder diesel engine was also examined by Obodeh et al. The objective of this work was to employ an ANN to reduce emissions from diesel engines. The artificial neural network was trained with experimentally obtained data and used to predict diesel emissions at a variety of operating scenarios. The backwards propagation learning algorithm was used to train the neural network, which was constructed with the multiplayer preceptron structure. Three outputs were desired for this research, CO, NOx, and PM emissions. An individual multilayer perceptron neural network was constructed for each desired output, this allows for better accuracy since each neural network is focused on its specific output. Data sets were available for 33 different operation scenarios, 23 of these data sets were used for training, and the remainders were employed for validation. It was determined that the neural network produced acceptable results, in agreement with standard emissions levels, and therefore showing that neural networks are applicable in predicting engine emissions [44].

Neural networks have also been applied to predicting failures and reliability of vehicle engines. Neural networks are selected for this application because they have the ability to learn based on past reliability indices and failure that will likely occur in the future based on this history. For this application it was determined that the radial basis function structure of the neural network was optimum, due to its employment of a local network, not global networks as used in the multilayer perceptron method. Using the local network rather than the global networks allows the program to use single sets of processing units, which are individually applicable to a specified region of the input space. This structure allows the program to determine if a given input is within or outside of the trained set of data, which distinguishes it from the multilayer perceptron method. The analysis and testing determined that neural networks are applicable to predicting failure in engine systems. The neural networks resulted in more accurate outputs than that of the currently employed linear models. This research also showed that the radial basis function method produced more reliable data compared to the feed-forward multilayer perceptron method [46]. 
A Study by Clark et al. determined if diesel engine emissions could accurately be predicted by a model that was developed using data and information from a different test schedule. Two modeling methods were evaluated, an artificial neural network method and a method that employed a model derived from continuous power data. The model based on continuous power data is a conventional approach to emissions modeling. In this conventional model it was common practice to model $\mathrm{NOx}$ and $\mathrm{CO}_{2}$ emissions as steady-state, this approach may become problematic as newer technologies such as exhaust gas recirculation (EGR) become more prevalent. It is not accurate to model $\mathrm{CO}$ and $\mathrm{PM}$ emissions with a steady-state model since transient effects are introduced as a result of the turbocharger. In this research only NOx and $\mathrm{CO}_{2}$ emissions were modeled with the conventional continuous data based method. The conventional means of modeling exhaust emissions was either based on continuous speed or power data. It was determined that the artificial neural network provided more accurate results when compared with the conventional modeling methods. The neural network was trained with data acquired from a specific test schedule, and then the model was used to predict emissions resulting from a different test schedule. The inputs for this model were axle speed, the first derivative of axle speed, the second derivative of axle speed, torque, and the first derivative of torque, and the second derivative of torque. The output of the model was the emission value of NOx. Through analysis it was determined that it is possible to accurately predict NOx emissions associated with a certain test cycle, with a neural network that has been trained with a different test cycle [11].

Researchers at an university in Spain have examined the feasibility of applying artificial neural networks to optimize engine parameters with the objective of reducing exhaust emissions to meet the more strict standards set to come in the future. To acquire the necessary training data, stationary tests were conducted on a single cylinder, diesel engine. For the neural network seven inputs were measured, including engine speed, air mass intake, fuel injection pressure, fuel mass injected, initial injection angle, EGR percentage, and nozzle diameter. NOx emissions, PM emissions, and fuel consumption were the three desired outputs of the neural network. The structure of the model in the research consisted of three separate neural networks, rather than one inclusive network. A network was constructed to predict each desired output, this method allows for the networks to learn and adapt to specifically predict each output. If a single neural network 
was employed, the variable with the least accuracy would hinder the accuracy of the prediction values for all outputs. It was determined that neural networks were applicable to engine parameter optimization as a function of engine emissions. The comparison of the outputs of the neural networks to measured values show that neural networks can accurately predict NOx emissions and fuel consumption, however a different set of inputs is necessary to better predict PM emissions [47].

Hashemi et al. have researched predicting heavy duty diesel engine emissions by employing an ANN. Six tractor trailer vehicles, equipped with varying engines, were tested on a chassis dynamometer and the recorded data were employed as training data for an ANN. The ANN predicted the quantities of $\mathrm{CO}_{2}, \mathrm{CO}, \mathrm{HC}$, and $\mathrm{NOx}$ emitted by heavy duty diesel engines. The neural network initially relied on 20 inputs, made up of engine parameters and their derivatives, however it was determined that similar results could be produced in less computational time with 14 inputs. While optimizing the model, the number of inputs was decreased and test compilations were completed in order to determine which input had the greatest impact on the outputs. It was determined that the predicted emission values were most strongly a function of the dispersed speed. The first and second derivatives of torque were also key factors in the emission values that were obtained as outputs.

In this research the neural network was trained with data that was acquired during testing of the Highway cycle, and was applied to predict emissions resulting from the CSHVR and UDDS cycles. Further research used the CSHVR and the UDDS cycle to train the network, and then used that network to predict emission values for other cycles. It was determined that the network trained with the Highway cycle, produced the most accurate results when predicting emissions expected from other cycles. This differing level of accuracy was attributed to the fact that the Highway cycle included acceleration, deceleration, and highway speed events. This research determined that when training an ANN it is best to use data that has been acquired over a range of driving conditions. It has also been shown that neural networks are applicable in estimating $\mathrm{CO}_{2}$ and NOx emissions. The $\mathrm{HC}$ emission predictions were not accurate due to the selection of parameters for this network. This network was based on power, speed, and torque, while it has been shown that $\mathrm{HC}$ emissions are dependent on operating temperature and transient behavior. 
This particular neural network also proved to be a poor predictor of CO emissions [50]. Traver et al. also documented difficulty in accurately predicting $\mathrm{HC}$ and $\mathrm{CO}$ emissions using neural networks [51]. This was attributed to the fact that $\mathrm{CO}$ is reliant on transient events and rapid acceleration events. The model was able to predict positive spikes in $\mathrm{CO}$ emissions at expected times; however the actual predicted values were not accurate. Brace was able to produce a neural network model that accurately accounted for rapid changes in engine operating conditions, which resulted in more accurately predicted values of $\mathrm{CO}$ emissions [52]. The research determined that artificial neural networks show potential for being employed in predicting emission inventories; however appropriate training data and input parameters must be employed [50].

Thompson et al. have shown that an artificial neural network is applicable to predicting torque and exhaust emissions for heavy duty diesel engines on the FTP and two random cycles. This neural network system consists of pre-processing and post processing. Before the data is applied to the neural network it is normalized and filtered. This pre-processing results in a more stable model, and a reduction in data noise. The outputs of the neural network are then filtered and denormalized. It was determined that $\mathrm{NOx}$ and $\mathrm{CO}_{2}$ were most accurately modeled by the network. Error was introduced when NOx prediction is required during idle conditions, under these conditions the model under predicted the NOx emitted. The quantities of $\mathrm{CO}$ and $\mathrm{HC}$ were not accurately predicted by the neural network; however for $\mathrm{CO}$ the model was able to predict when major CO production events occurred [53].

\subsubsection{ANN Applied to Engine Optimization and Modeling}

Neural networks have been successfully applied to diesel engine related areas other than emissions prediction. Delagrammatikas et al. developed a neural network to simulate a heavy duty diesel engine with the objective of reducing in-vehicle engine design time. In this work the ability of a neural network to model a heavy duty diesel engine for optimization purposes was compared to commonly used high-fidelity models. It was determined that the neural network possessed greater stability than the current high-fidelity model, and produced results with less than six percent error compared to the high-fidelity baseline model. Through this research it was 
determined that neural networks are applicable in engine optimization and modeling situations and are comparable in accuracy and computational time to current high fidelity models [49].

Researchers have also worked on determining the viability of employing genetic algorithms and neural networks to aide in the optimization of diesel engine operations. Diesel engines were modeled and simulated with artificial neural networks, which could predict engine emissions and fuel consumption based on input engine operation characteristics [37]. The specific exhaust constituents that were modeled were $\mathrm{HC}, \mathrm{CO}, \mathrm{PM}$, and NOx. The fuel consumption was evaluated on a brake-specific scale. A secondary objective was to arrive at an optimal combination of engine input parameters that would reduce the fuel consumption, while still complying with emission standards. Attempts at optimizing engine parameters via numerical modeling and techniques have been widely documented and have been determined to be applicable in limited scenarios. Numerical optimization algorithms can be affected by local extrema, and discontinuities in the models functions. This work examined artificial neural networks for optimization of the engine parameters, due to their relative immunity to functional discontinuities such as non-linear behavior and local minima and maxima. Table 2.4.6.1 shows each of the input variables associated with the artificial neural network (ANN). These parameters were varied independently in order to construct 440 test cases that were evaluated by the ANN. This study determined that ANN and genetic algorithms can be effectively employed to model engine operations and emissions, and be used to optimize engine operations to meet emissions and fuel consumption targets [37].

Table 2.4.6.1: Engine Operating Parameters Used as Inputs to Genetic Algorithm [37].

\begin{tabular}{|c|c|}
\hline Operating Parameters & Variable \\
\hline Engine Speed & $\mathrm{N}$ \\
\hline Fuel Mass Injected & $\mathrm{Mf}$ \\
\hline Air Mass & $\mathrm{Ma}$ \\
\hline Exhaust Gas Recirculation & $\mathrm{EGR}$ \\
\hline Injection Pressure & $\mathrm{IP}$ \\
\hline Start of Pilot Injection & SOIP \\
\hline Start of Main Injection & SOIM \\
\hline Intake Temperature & Tint \\
\hline Water Temperature & Tw \\
\hline
\end{tabular}


Tutuncu et al. also determined through research that artificial neural networks were applicable to diesel and gasoline engine modeling. The objective of this work was to model exhaust emissions and performance of gasoline and diesel fueled internal combustion engines via an artificial neural network. Since the diesel engine model is most relevant to the current research topic, only it will be addressed here. The ANN model required five inputs to produce six outputs. RSquared values for each of the modeled characteristics were over 0.99 when the artificial neural network results were compared to experimental data [41].

Other research has been directed towards simulating the rate of combustion in diesel engines during transient operation with an empirical model. An artificial neural network was selected for the model due to its speed of computation and application to nonlinear phenomena. To verify the results of the model, the model outputs were compared to experimentally obtained results. The neural network modeled the rate of heat released from a turbocharged diesel engine during transient conditions, and required the following inputs: in-cylinder pressure, air and fuel mass flow, EGR rate, boost pressure, exhaust manifold pressure, and intake and exhaust gas temperatures. The output of the model was the rate of heat released based on the time segment that the valves were closed and the heat lost through the engine walls. In the training of the artificial neural network, the input and output data was normalized so that the neural network learned about differences and not actual values. This normalization helped the neural network learn to predict more accurately. The learning method employed for the neural network was back propagation, and the model was structured in a multilayer perceptron manner. The multilayer peceptron structure consisted of one hidden layer and between one and eight neurons. After comparing the outputs of the neural network model to experimental results it was determined that neural networks are applicable to any engine transient operation situation [45].

An artificial neural network has also been designed to predict specific fuel consumption and exhaust temperature associated with a heavy duty diesel engine. The neural network developed for this task consisted of three inputs and two outputs. The inputs employed by the network were engine speed, brake mean effective pressure, and injection timing. The outputs were the desired objectives discussed above, brake specific fuel consumption, and exhaust temperature. When the neural network outputs were compared to experimental data, a difference of less than two 
percent was achieved. This work shows that with few inputs, neural networks can accurately predict exhaust temperature and fuel consumption [48]. 


\section{Vehicle Testing and Data Collection}

It should be noted that the data employed in this research was acquired through previous research efforts of CAFEE. Acknowledgement and appreciation is given to the engineers, staff, faculty, and graduate students that were associated with the mobile emissions measurement system research, and data acquisition which occurred at the engine and emissions research laboratory.

\subsection{In-use Data Acquisition}

The in-use data employed in the training and validation of the ANN developed in this research was obtained with WVU's Mobile Emission Measurement System (MEMS). The system was installed on multiple vehicles equipped with various engines in order to record in-use emission data for different routes. The vehicles being examined were equipped with ambient sensors in order to measure ambient temperature, ambient pressure, and relative humidity. The vehicles were also equipped with a global positioning system (GPS) to record the vehicle speed and location. Exhaust gas analyzers were used to measure the quantities of $\mathrm{NOx}$ and $\mathrm{CO}_{2}$ which were being emitted. Data acquisition systems were interfaced with the engine control module (ECM) in order to record engine speed and torque values. Figure 3.1.1 shows a schematic of the MEMS setup [62]. 


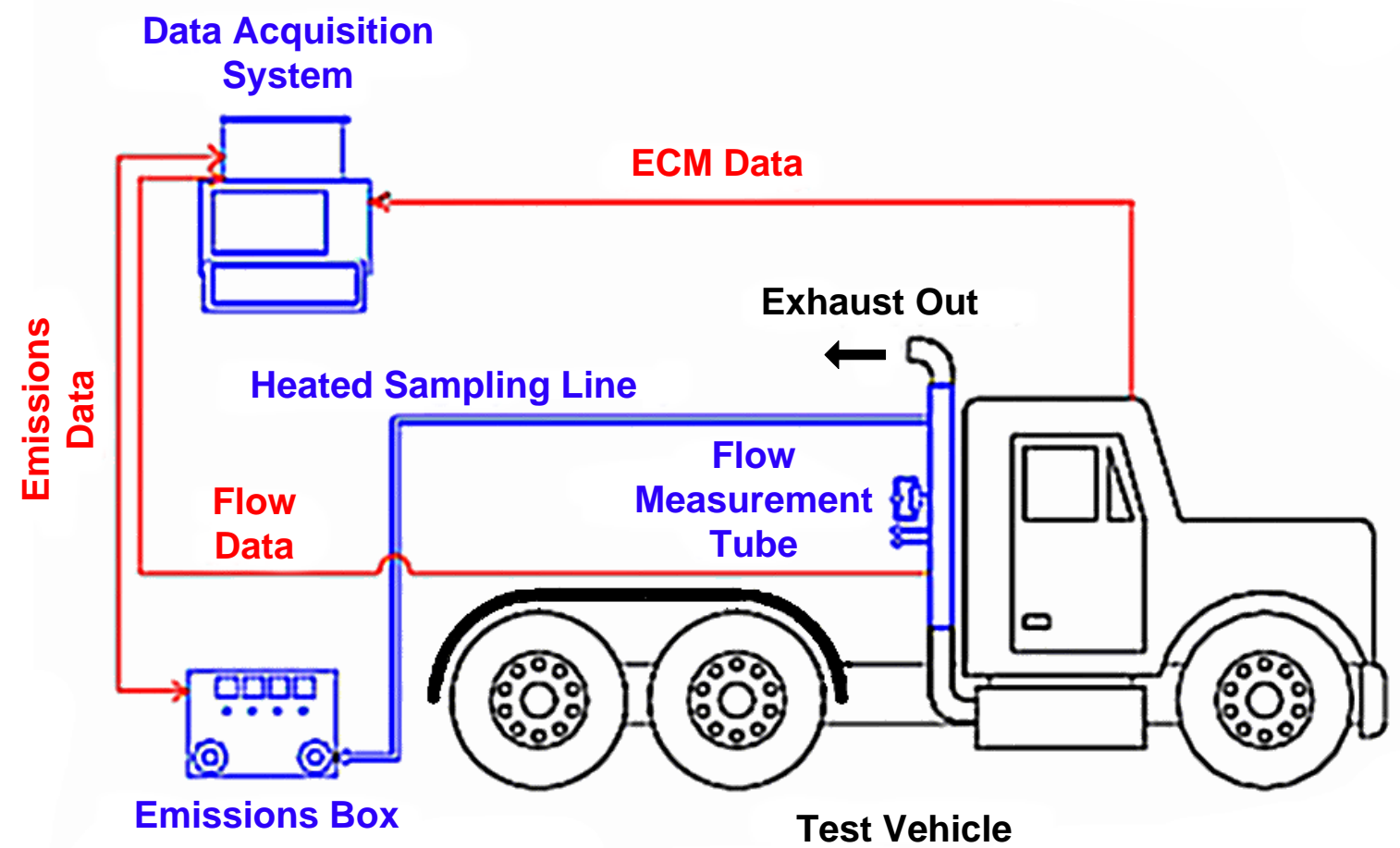

Figure 3.1.1: Schematic of MEMS [62]

\subsection{Test Engines}

A variety of engines from different manufacturers were tested with the WVU's Mobile Emissions Measurement System. For this research data, a $400 \mathrm{hp}$ engine from Manufacturer A was employed. Table 3.2.1 displays the specifications for this engine. The Manufacturer A was subjected to both in-use and engine dynamometer emissions testing. The engine torque and speed plots associated with the routes shown in the following sections of this chapter were constructed based on Manufacturer A $400 \mathrm{hp}$ engine data. Tables 3.2.2 and 3.2.3 display the specification associated with the engines and vehicles employed from Manufacturer B and Manufacturer C. 
Table 3.2.1: Manufacturer A Engine and Vehicle Specifications

\begin{tabular}{|c|c|}
\hline Manufacturer & Manufacturer A \\
\hline Power Rating & $400 \mathrm{hp}$ \\
\hline Year & 1995 \\
\hline Engine Configuration & Inline -6 \\
\hline Transmission & Manual \\
\hline Test Weight Range & $78,320-78,480 \mathrm{lbs}$ \\
\hline
\end{tabular}

Table 3.2.2: Manufacturer B Engine and Vehicle Specifications

\begin{tabular}{|c|c|}
\hline Manufacturer & Manufacturer B \\
\hline Power Rating & $525 \mathrm{hp}$ \\
\hline Year & 2002 \\
\hline Engine Configuration & Inline -6 \\
\hline Transmission & Manual \\
\hline Test Weight & $66,240 \mathrm{lbs}$ \\
\hline
\end{tabular}

Table 3.2.3: Manufacturer C Engine and Vehicle Specifications

\begin{tabular}{|c|c|}
\hline Manufacturer & Manufacturer C \\
\hline Power Rating & $345 \mathrm{hp}$ \\
\hline Year & 2002 \\
\hline Engine Configuration & Inline -6 \\
\hline Transmission & Manual \\
\hline Test Weight & $58,140 \mathrm{lbs}$ \\
\hline
\end{tabular}

\subsection{Vehicle Routes}

Vehicles equipped with the MEMS travelled specified routes, and data were recorded for the duration of the travel. For this research data obtained on the Bruceton Mills, WV route and the Washington, PA route were used.

\subsubsection{Bruceton Mills, WV Route}

The Bruceton Mills, WV route began and ended in the Sabraton area of Morgantown, WV. The vehicle turned around at a designated location, Bruceton Mills, WV, on Interstate 68. The majority of the travel occurred on Interstate 68, where the posted speed limit is $70 \mathrm{mph}$, however the return trip includes required speed reductions to $50 \mathrm{mph}$ for descending hills and a required 
brake check stop. The travel from Sabraton, WV to Bruceton, Mills, WV and back to Sabraton, WV was 39.7 miles in its entirety [63]. Figure 3.3.1.1 depicts the Bruceton Mills, WV route [65]. The travel was split into two routes, the route from Sabraton, WV to Bruceton Mills, WV, and the return route from Bruceton Mills, WV to Sabraton, WV.

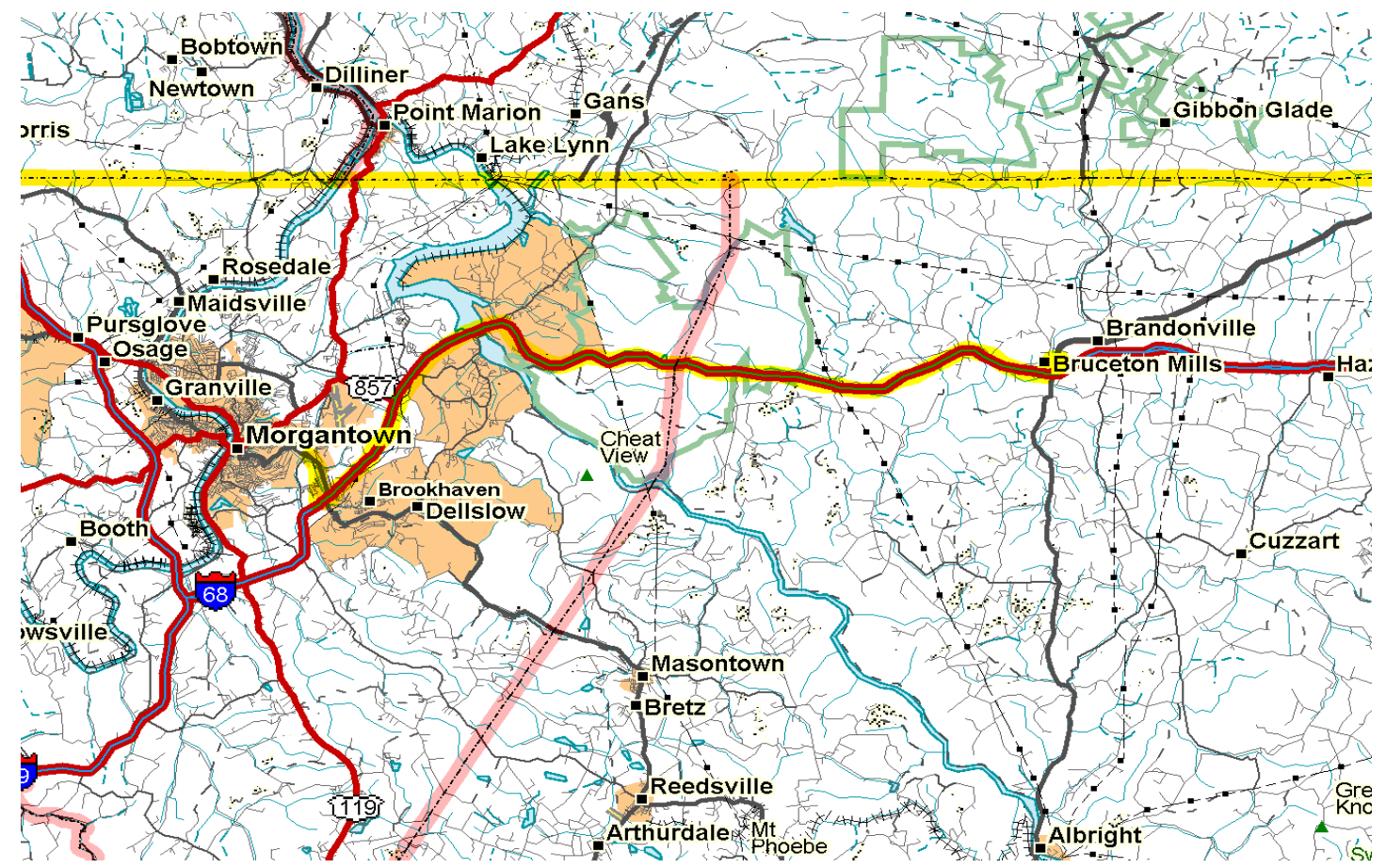

Figure 3.3.1.1: Map of Bruceton Mills, WV Route [65]

Figures 3.3.1.2 and 3.3.1.3 show the engine speed and torque, respectively, associated with the route from Sabraton, WV to Bruceton Mills, WV. The engine speed and torque associated with the return route from Bruceton Mills, WV to Sabraton, WV are shown in Figures 3.3.1.5 and 3.3.1.6, respectively. 


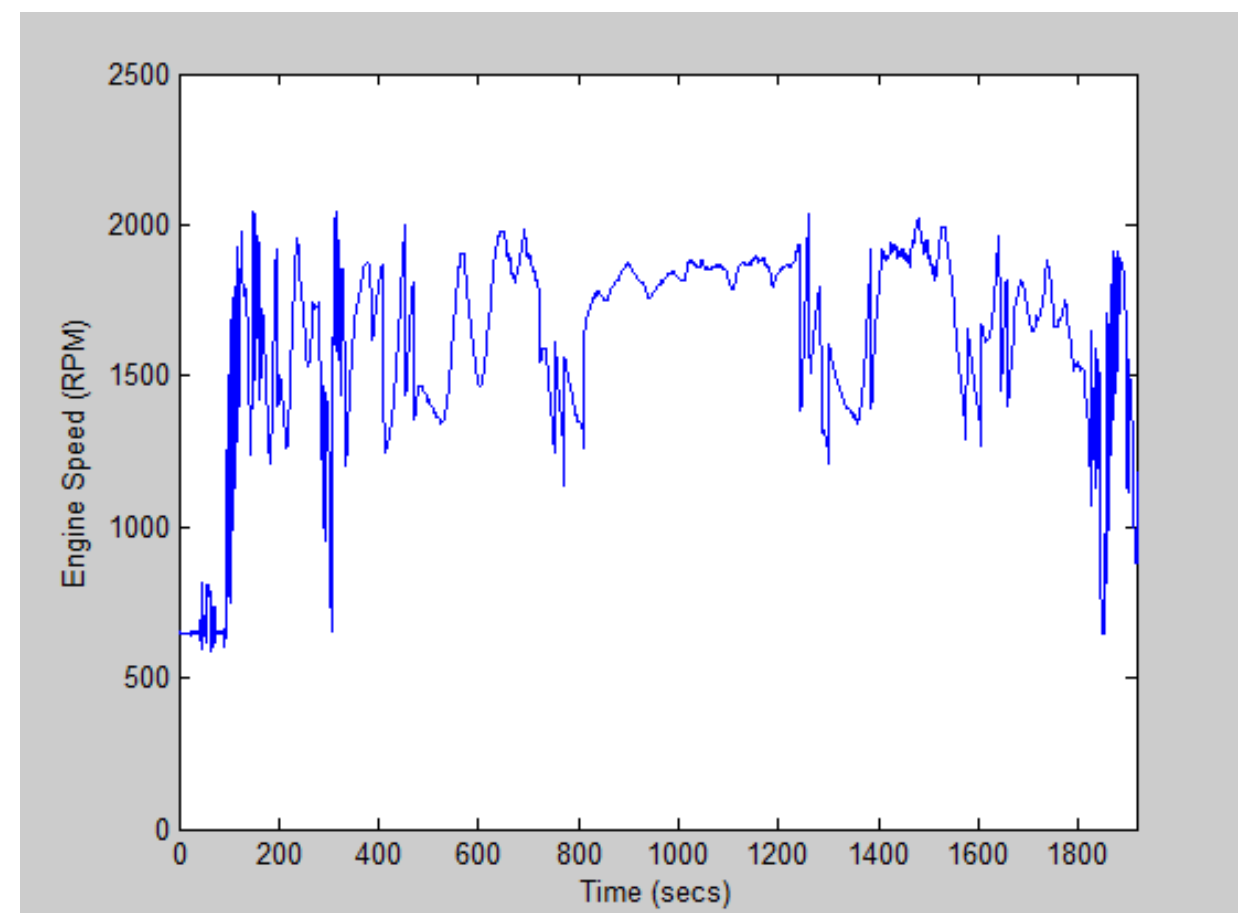

Figure 3.3.1.2: Engine Speed for the Sabraton, WV to Bruceton Mills, WV Route with Manufacturer A Engine

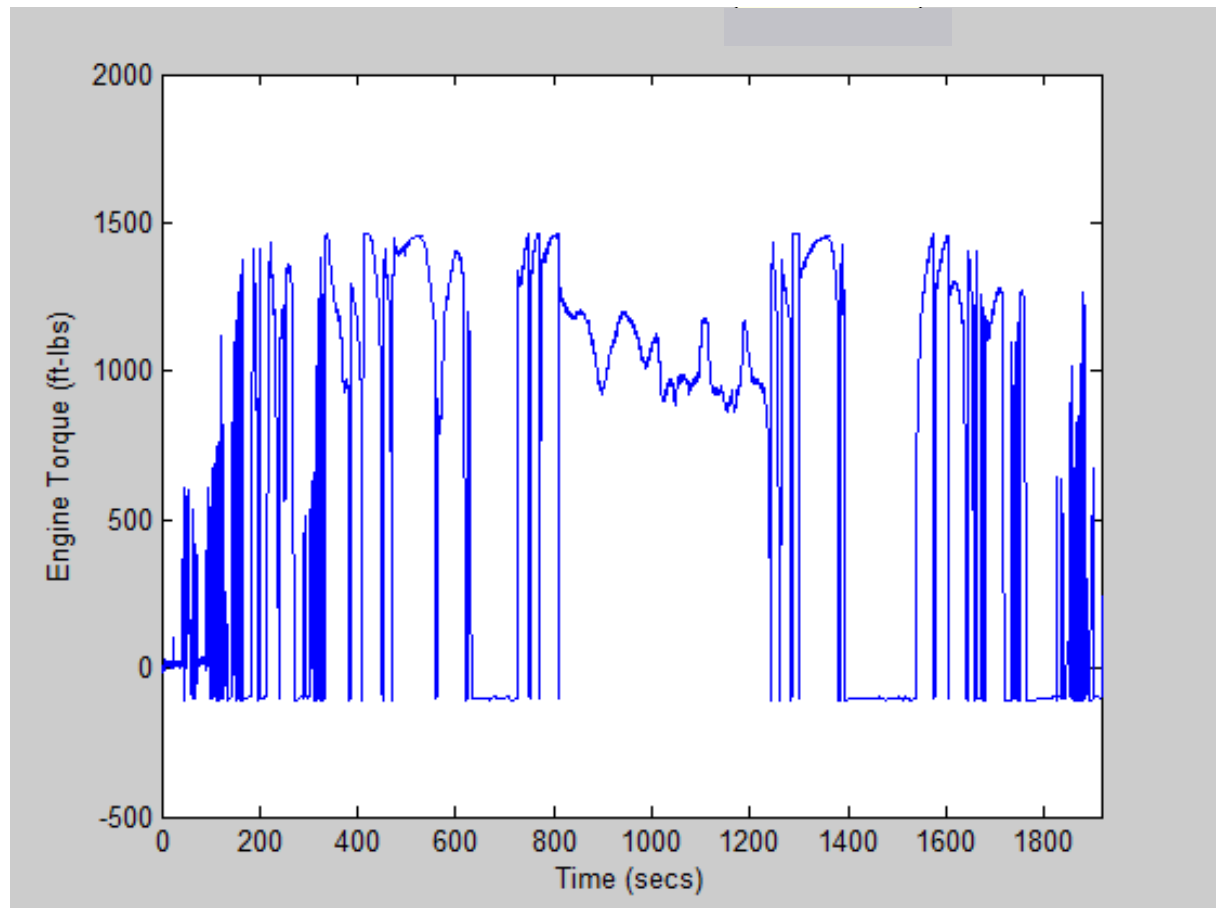

Figure 3.3.1.3: Engine Torque for the Sabraton, WV to Bruceton Mills, WV Route with Manufacturer A Engine 
Figure 3.3.1.4 displays the road grade associated with the route from Sabraton, WV to Bruceton Mills, WV. It is shown that the road grade ranges from $-15 \%$ to $7 \%$. The travel which occurred between 800 and 1300 seconds was the longest continual incline travel that occurred in any of the routes which were examined. The road grade determined to be $-15 \%$ was due to a missing data point or measurement error in the pressure data, therefore the spike shown at 2050 seconds was due to the application of a moving average including the inaccurate data point.

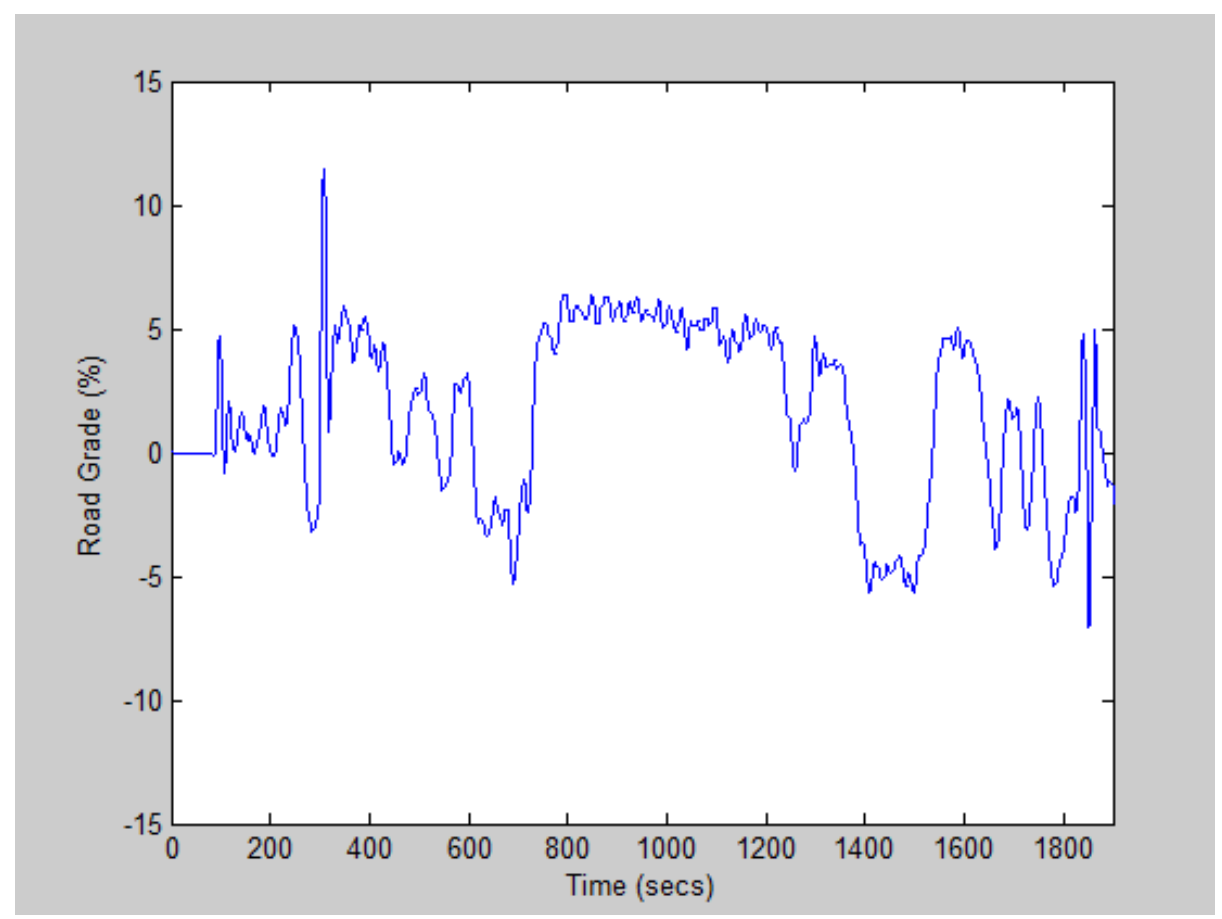

Figure 3.3.1.4 Road Grade from Sabraton, WV to Bruceton Mills, WV with Manufacturer A Engine 


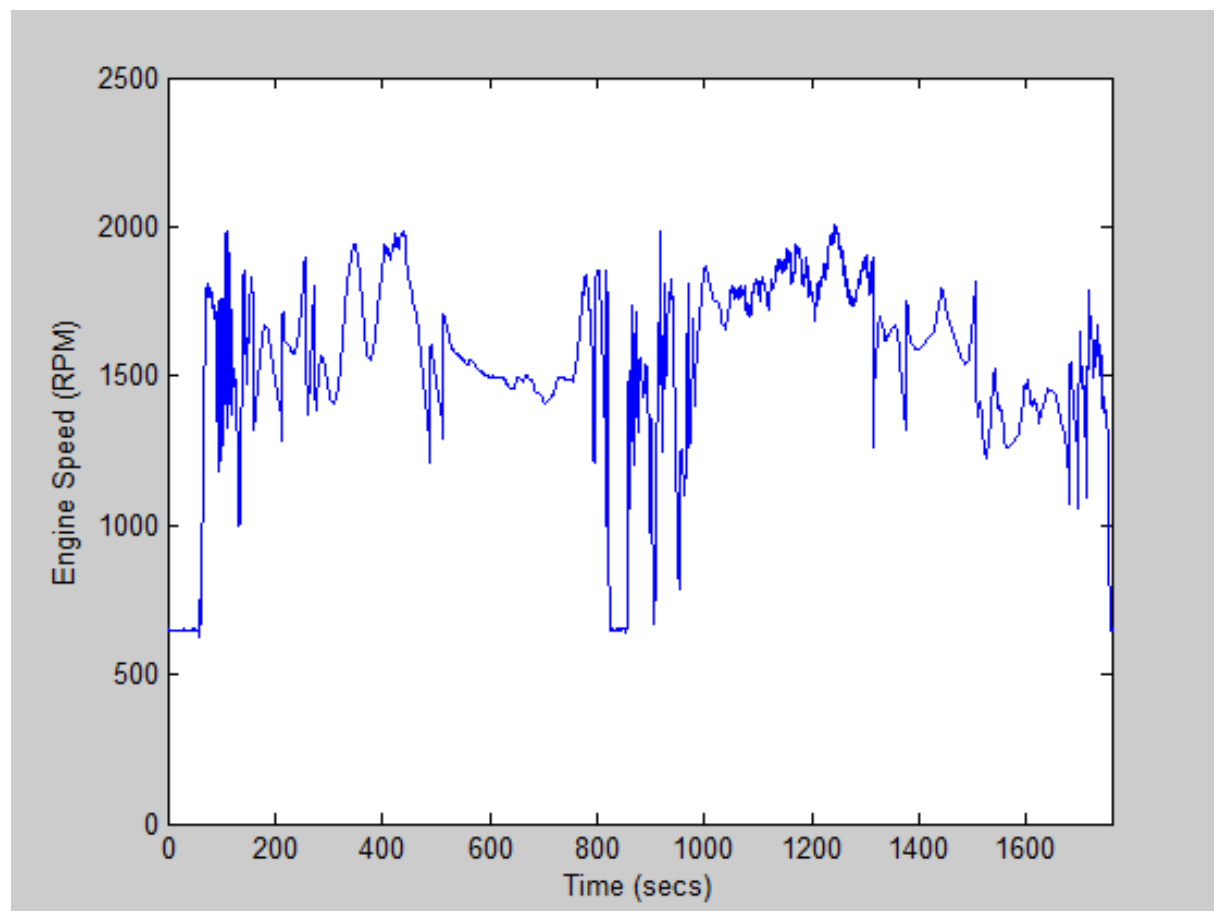

Figure 3.3.1.5: Engine Torque for Bruceton Mills, WV to Sabraton, WV Route with Manufacturer A Engine

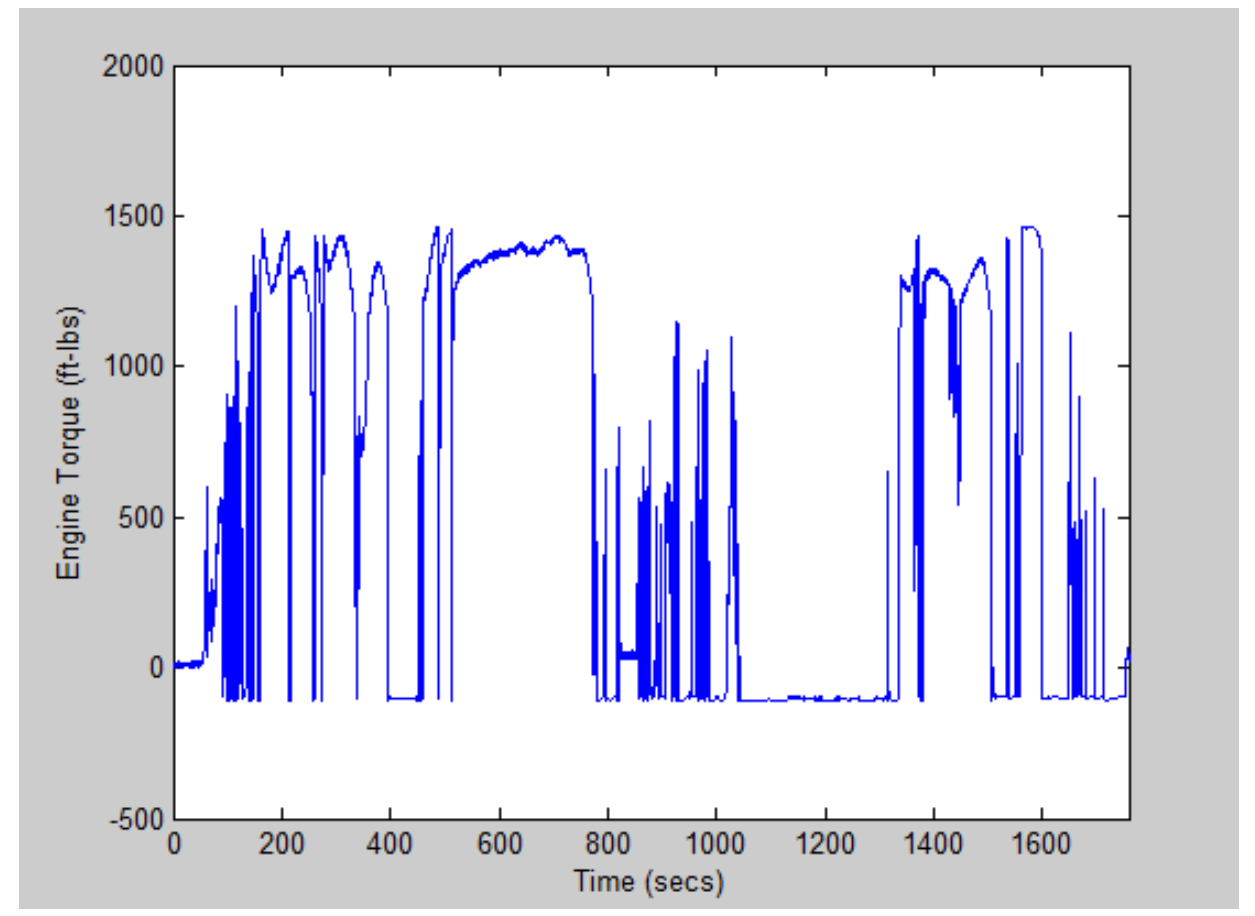

Figure 3.3.1.6: Engine Torque for Bruceton Mills, WV to Sabraton, WV Route with Manufacturer A Engine 
The road grade associated with the travel from Bruceton Mills, WV to Sabraton, WV is shown in Figure 3.3.1.7. The road grade ranged from $-14 \%$ to $14 \%$. The portions of the route between 400 and 800 seconds, and 1000 and 1300 seconds represent periods of ascent and descent, respectively.

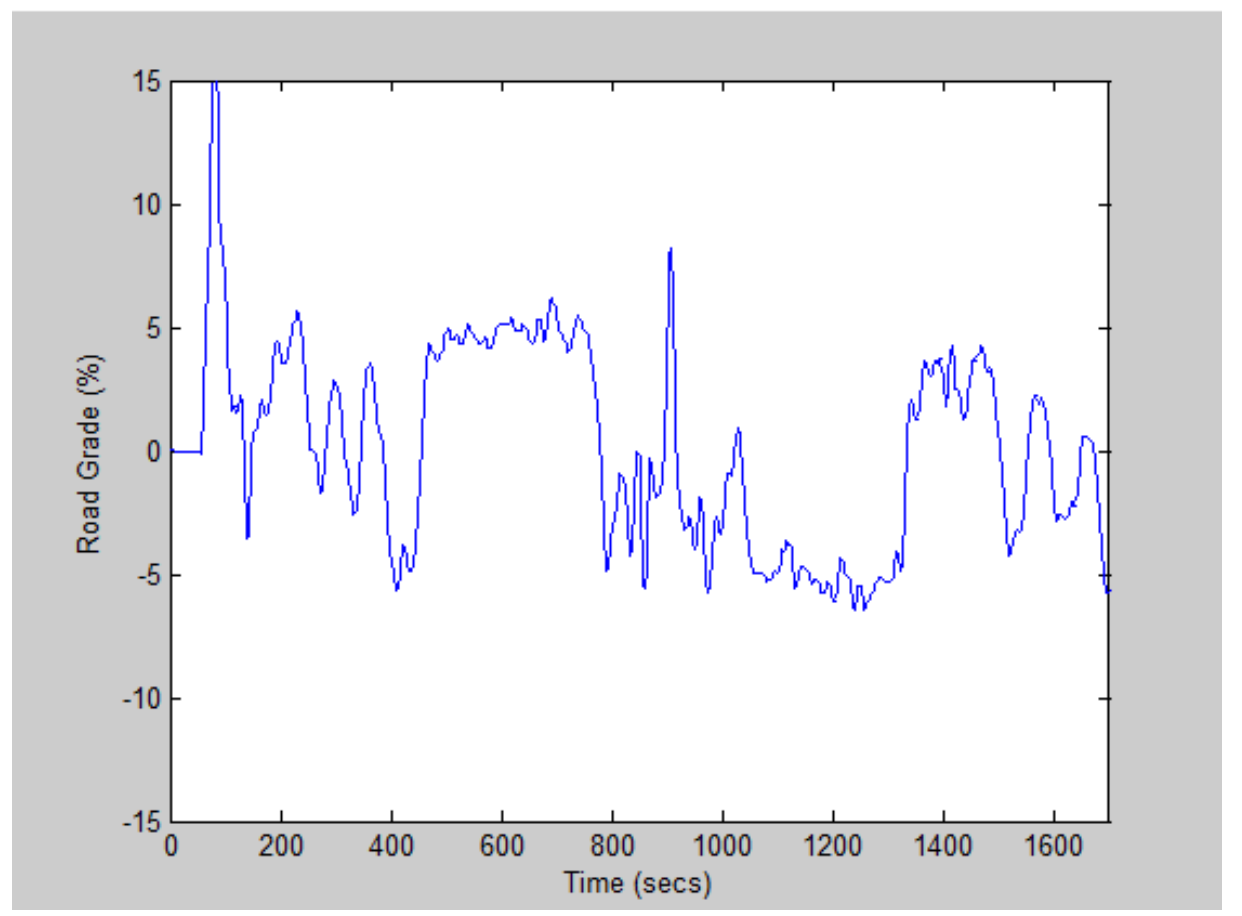

Figure 3.3.1.7 Road Grade From Bruceton Mills, WV to Sabraton, WV with Manufacturer A Engine

\subsubsection{Washington, PA Route}

The second route, designated the Washington, PA route, began in Washington, PA and concluded at the first rest area in West Virginia. This route was a combination of suburban and interstate driving scenarios. The speed limits on this route varied from $25-45 \mathrm{mph}$ in the suburban areas to $55-65 \mathrm{mph}$ on the highway portions of the route. From Washington, PA the vehicle traveled on United States Route 19 north, which incorporated suburban driving conditions. The vehicle then traveled on Pennsylvania State Route 51 to Interstate 279 south, and then proceeded on Interstate 79 south until the West Virginia rest stop was reached [63]. 
The Washington, PA route was split into three sections, Washington, PA 1, Washington, PA 2, and Washington, PA 3. Figures 3.3.2.2 and 3.3.2.3 show the engine speed and torque, respectively, associated with the Washington, PA 1 route. This route was a total of 12.1 miles, starting at Exit 19B on Interstate 79, and ending at the pull-over on Route 19, outside of Upper St. Claire, PA [65]. Figure 3.3.2.1 shows a map of the Washington, PA 1 portion of the Washington, PA route.

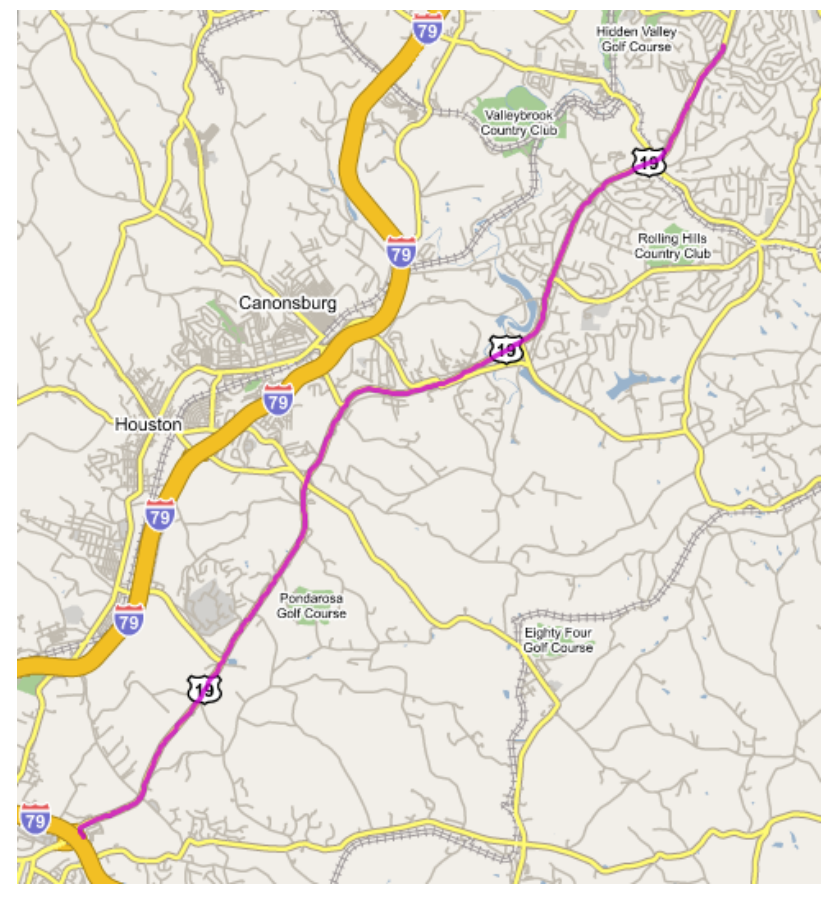

Figure 3.3.2.1: Washington, PA 1 Route [65] 


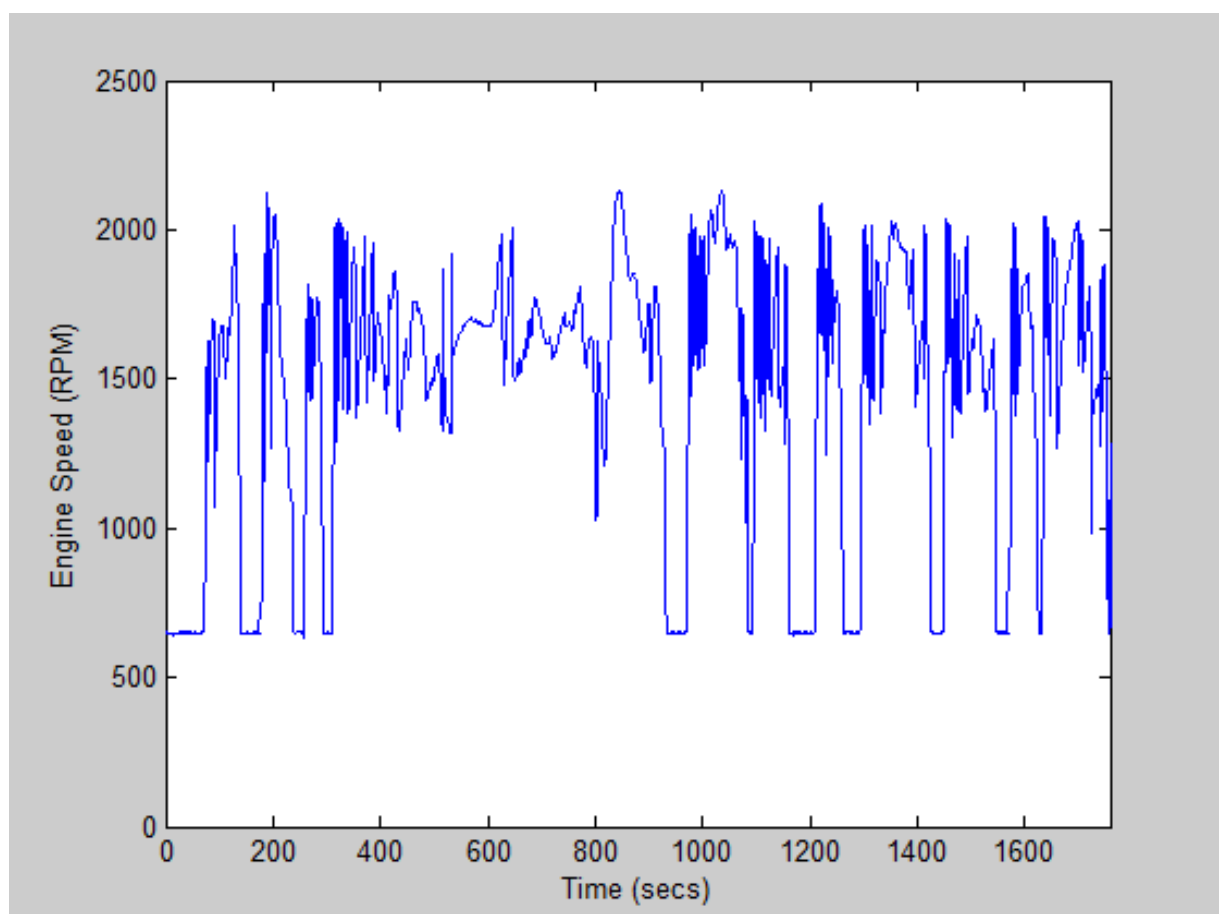

Figure 3.3.2.2: Engine Speed for Washington, PA 1 Route with Manufacturer A Engine

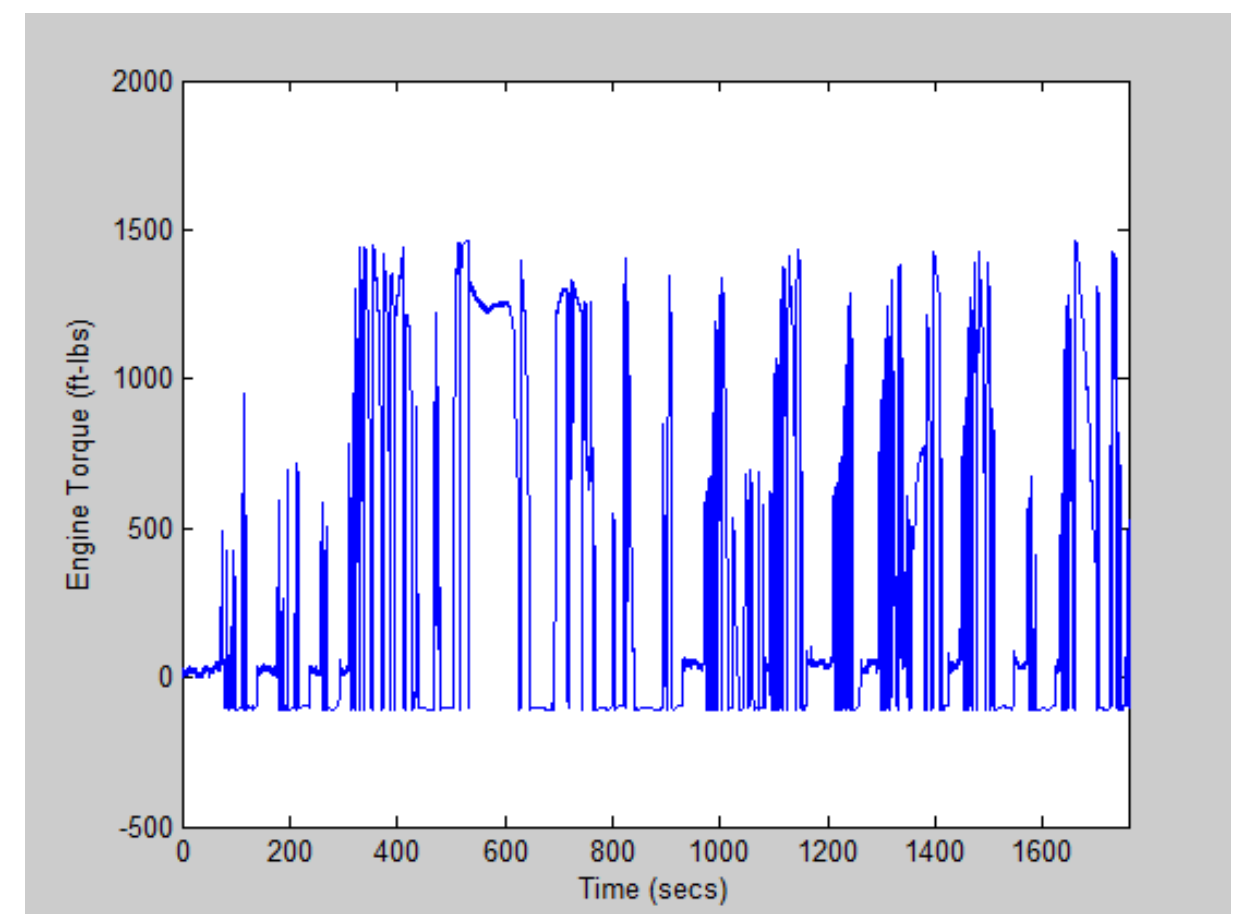

Figure 3.3.2.3: Engine Torque for Washington, PA 1 Route 
Figure 3.3.2.4 displays the road grade associated with the Washington, PA 1 route. It is shown that for the majority of the route the road grade is between $6 \%$ and $-6 \%$.

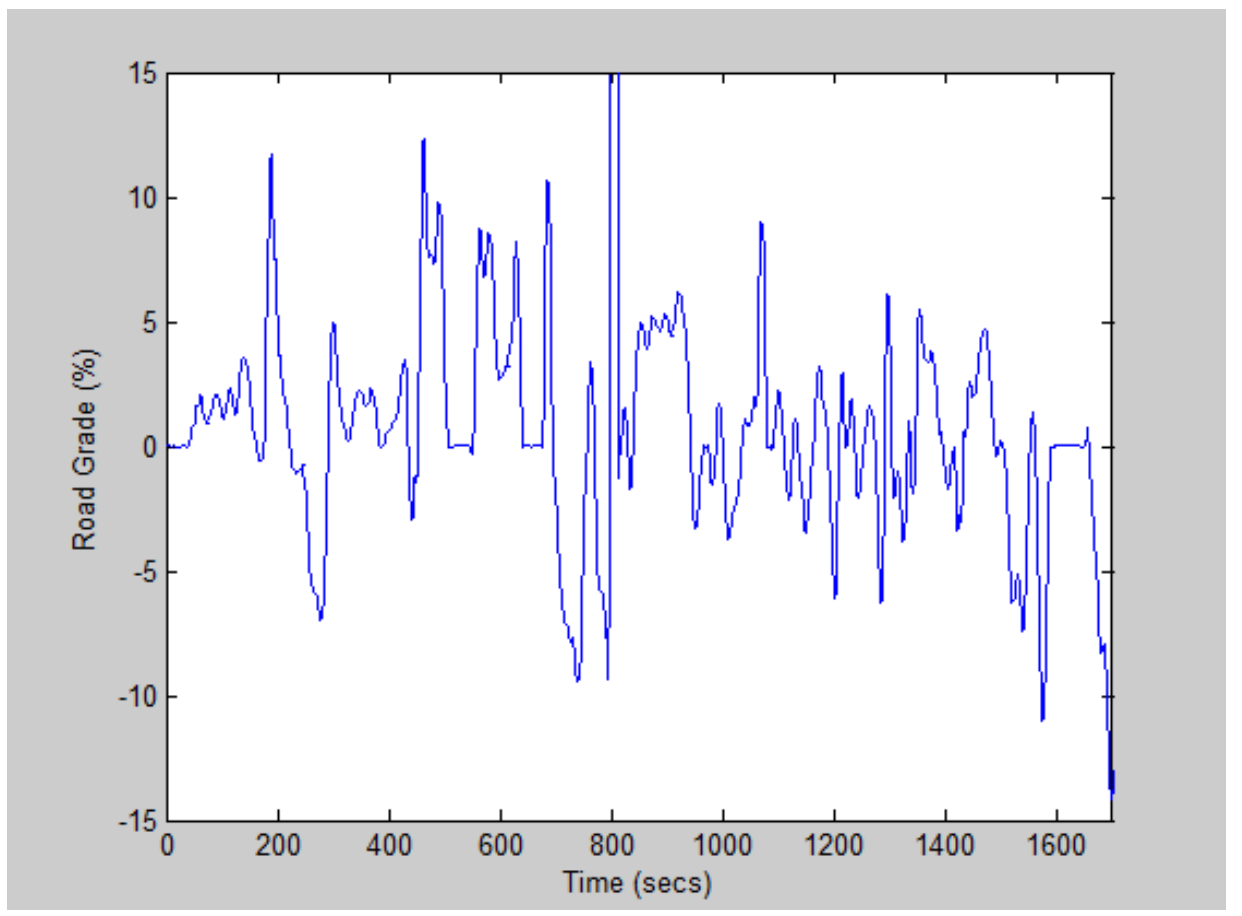

Figure 3.3.2.4: Road Grade for Washington, PA 1 Route with Manufacturer A Engine

The second portion of the Washington, PA route began where the Washington, PA 1 portion ended, at the pull-over area outside of Upper St. Clair, PA. The route then followed Route 19 to Interstate 279, passing through Mt. Lebanon, PA. The route then followed Interstate 279 to Interstate 79 South, until the rest area at Bridgeville, PA. The Washington, PA 2 portion of the Washington, PA route ended at the rest area at Bridgeville, PA. The total length of the Washington, PA 2 portion of the Washington, PA route was 23.1 miles [65]. Figure 3.3.2.5 shows a map of the Washington, PA 2 route. Figures 3.3.2.6 and 3.3.2.7 show the engine speed and torque, respectively, associated with the Washington, PA 2 route. 


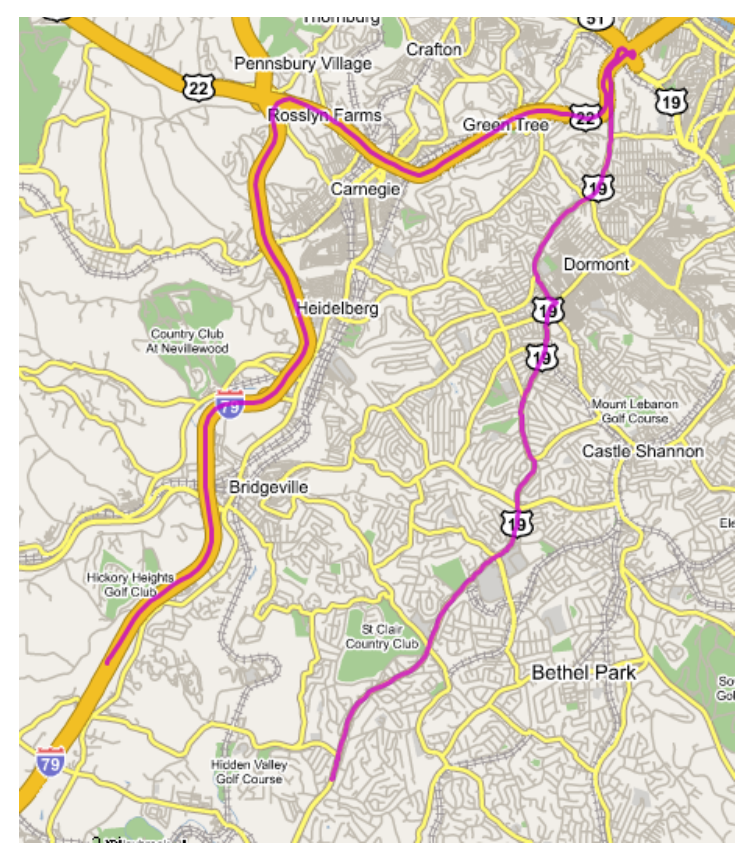

Figure 3.3.2.5: Washington, PA 2 Route [65]

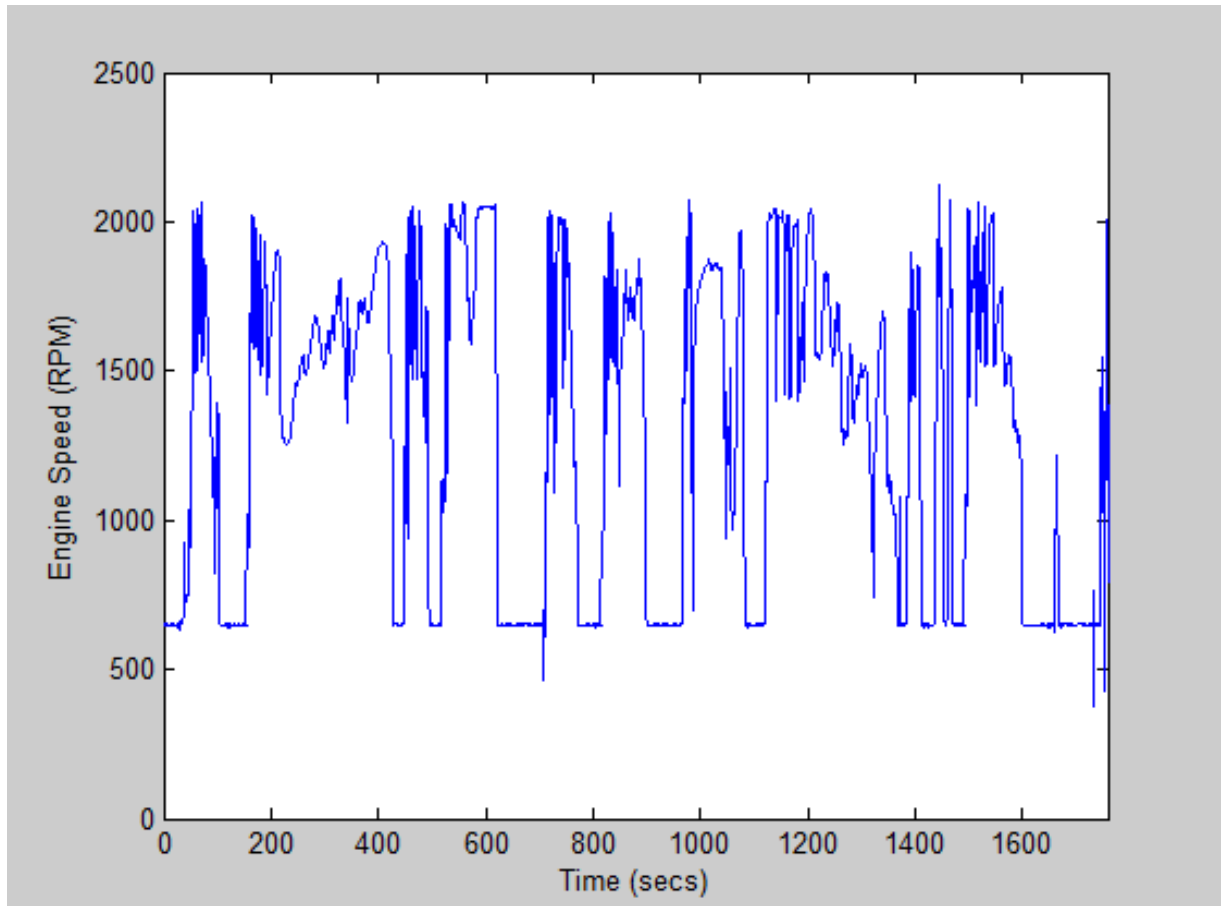

Figure 3.3.2.6: Engine Speed for Washington, PA 2 Route with Manufacturer A Engine 


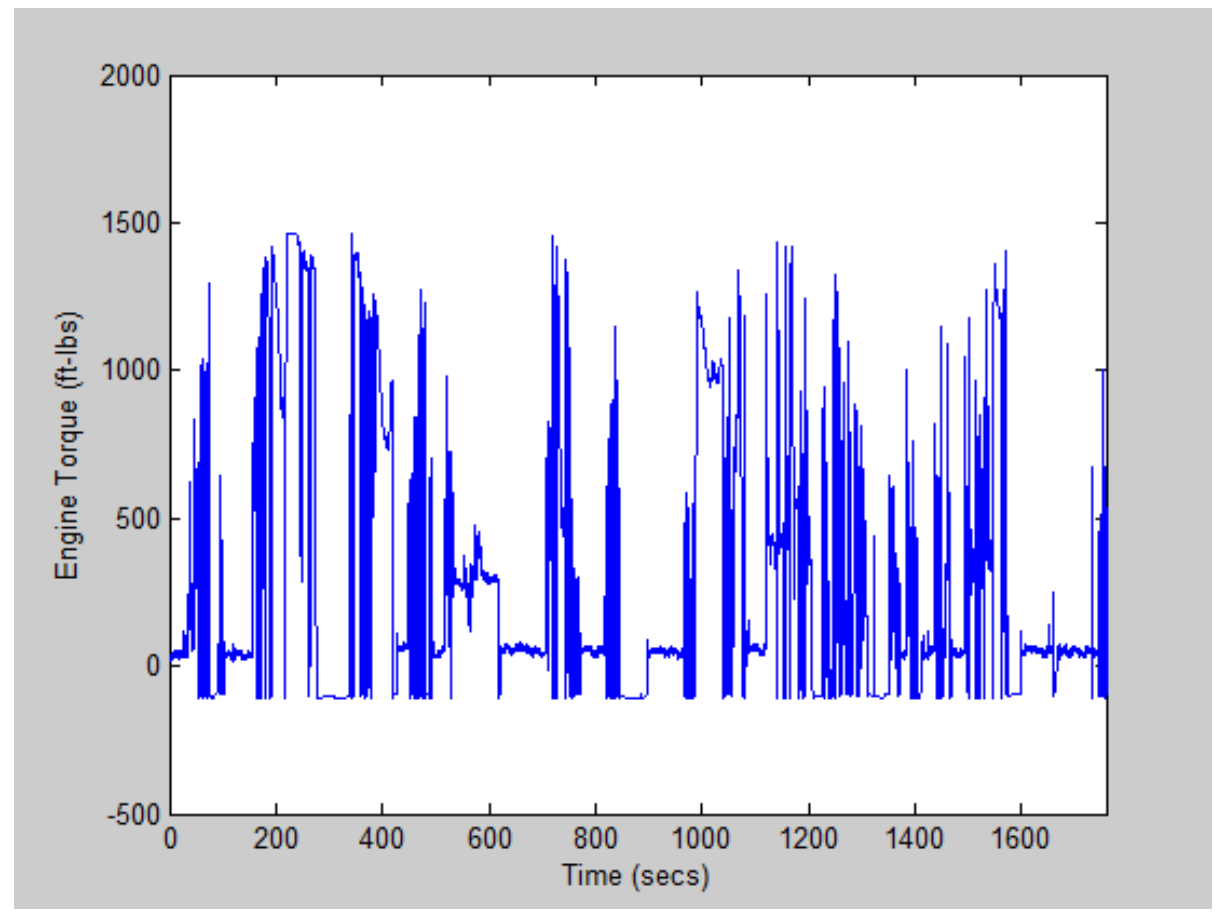

Figure 3.3.2.7: Engine Torque for Washington, PA 2 Route with Manufacturer A Engine

The road grade associated with the Washington, PA 2 route is shown in Figure 3.3.2.8. It is shown that the periods of incline and decline in this route are shorter than those experienced in the Bruceton Mills, WV routes. 


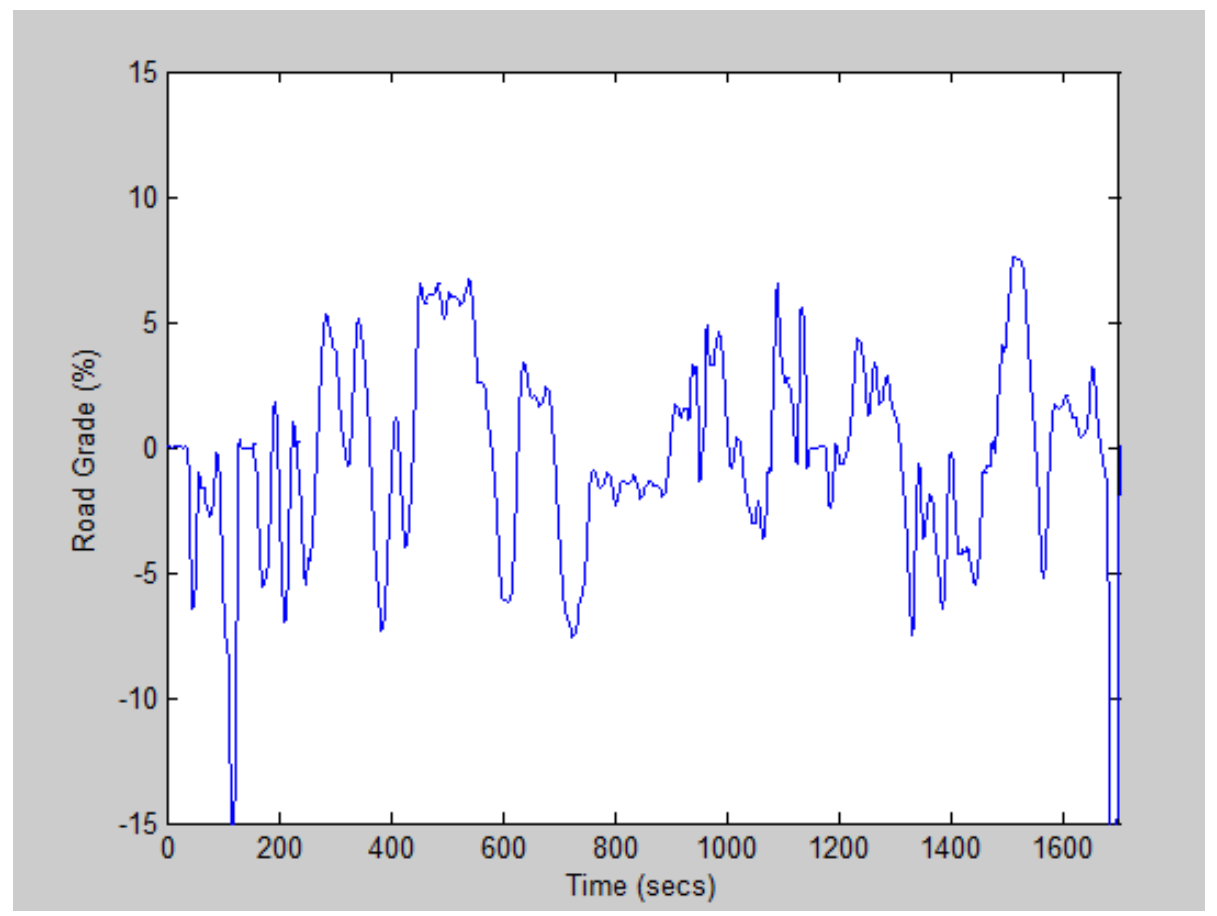

Figure 3.3.2.8: Road Grade for Washington, PA 2 Route with Manufacturer A Engine

The third portion of the Washington, PA route began where the Washington, PA 2 route ended, at the Bridgeville, PA rest stop. The Washington, PA 3 portion of the route followed Interstate 79 South until the first rest stop in West Virginia, which served as the ending location. The total distance traveled for the Washington, PA 3 route was 51.8 miles. The map shown in Figure 3.3.2.8 shows the Washington, PA 3 route, and then shows the route continuing until the vehicle returned to Sabraton, WV [65]. The engine speed and torque associated with the Washington, PA 3 route are shown in Figures 3.3.2.9 and 3.3.2.10, respectively. 


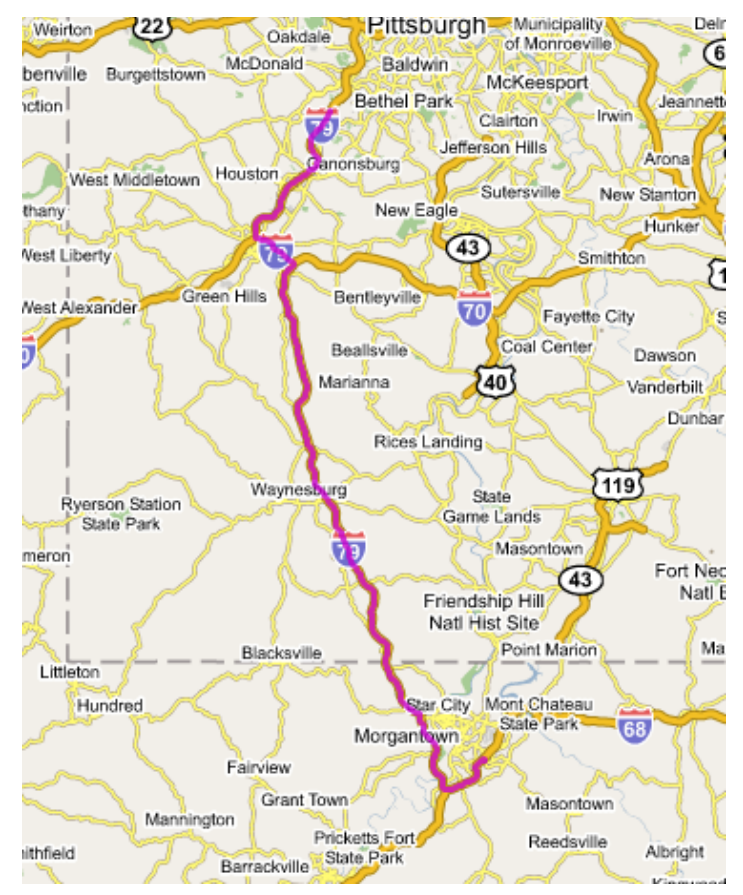

Figure 3.3.2.8: Washington, PA 3 Route [65]

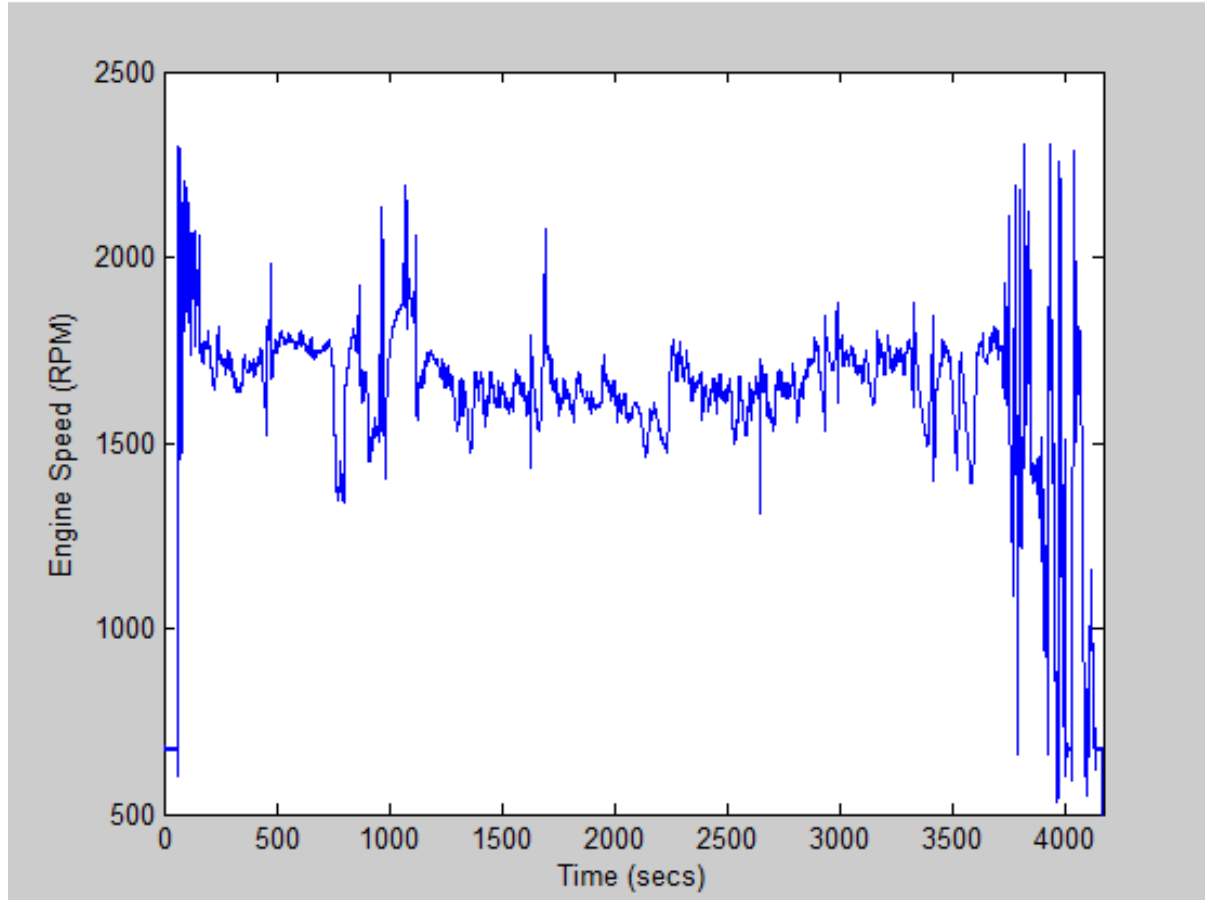

Figure 3.3.2.9: Engine Speed for Washington, PA 3 Route with Manufacturer B Engine 


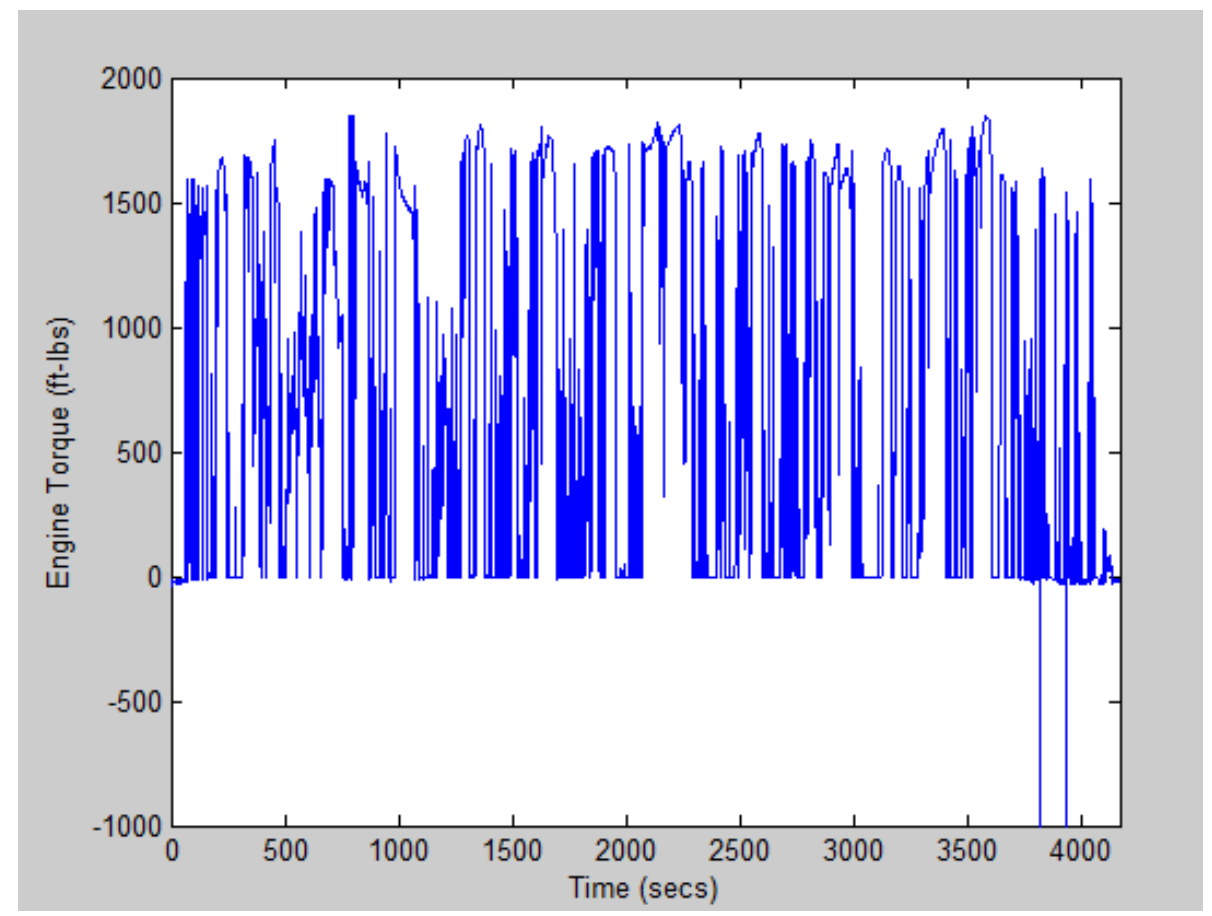

Figure 3.3.2.10: Engine Torque for Washington, PA 3 Route with Manufacturer B Engine

The torque values displayed in the previous figure at 3820 seconds and 3930 seconds were not representative of values experienced during the vehicle operation on the examined route. These two points were determined to be post-processing errors. The torque values plotted in the figure were calculated from the percentage load recorded by the ECU and the maximum torque. At these two data points the engine speed and torque recorded by the ECU were of the same order as the data points preceding and proceding the data points being examined. Figure 3.3.2.11 displays the road grade associated with the Washington, PA 3 route. The road grade ranged from $-14.5 \%$ to $13 \%$. 


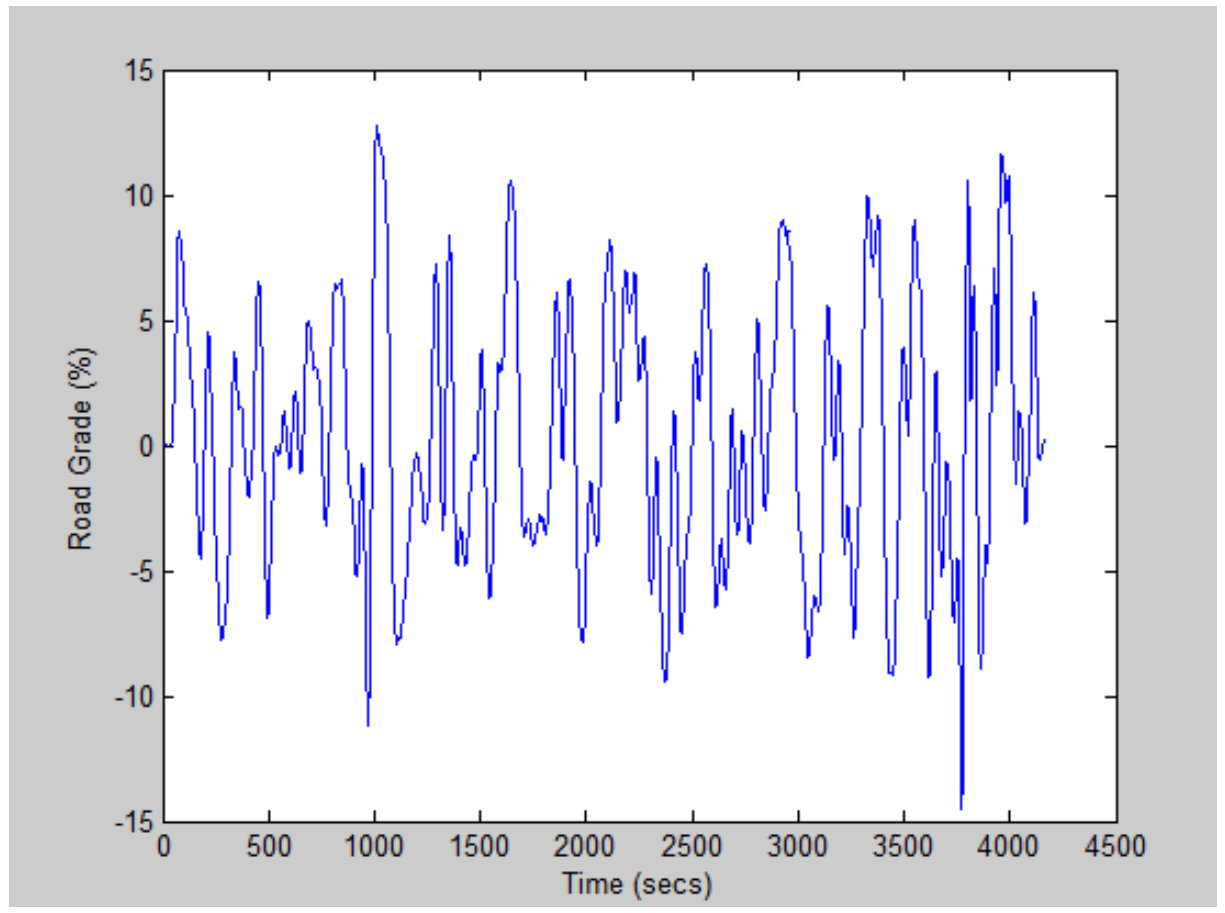

Figure 3.3.2.11: Road Grade for Washington, PA 3 Route

\subsubsection{In-Use Data Set Designations}

Throughout the remainder of this document the data sets that were used for training and verification are referred to using designated titles. Table 3.3.3.1 displays the data set titles and the routes they are associated with for the $400 \mathrm{hp}$ engine by Manufacturer A. The data sets titled Sab2Bruceton 1 and Sab2Bruceton 2 were acquired from a vehicle traveling from Sabraton, WV to Bruceton Mills, WV, while data sets Bruceton2Sab 1 and Bruceton2Sab 2 correspond to specific data sets that were taken during the return route from Bruceton Mills, WV to Sabraton, WV. The numbers after to data set designation title denote the repeated runs of the specific route. Each of the route in the table were run twice, the first run designated with a 1, and the second designated with a 2 . 
Table 3.3.3.1 Data Set Designations

\begin{tabular}{|c|c|}
\hline Data Set Designation & Route \\
\hline Sab2Bruceton 1 & Sabraton, WV to Bruceton Mills, WV \\
\hline Bruceton2Sab 1 & Bruceton Mills, WV to Sabraton, WV \\
\hline Sab2Bruceton 2 & Sabraton, WV to Bruceton Mills, WV \\
\hline Bruceton2Sab 2 & Bruceton Mills, WV to Sabraton, WV \\
\hline Wash PA1 1 & Washington PA 1 \\
\hline Wash PA1 2 & Washington PA 1 \\
\hline Wash PA2 1 & Washington PA 2 \\
\hline Wash PA2 2 & Washington PA 2 \\
\hline
\end{tabular}

\subsection{Engine Dynamometer Data Acquisition}

In addition to the data obtained by the MEMS projects, data obtained at the EERC at WVU was also employed in the development and verification of the ANN developed for this research. This facility was build in 1993 in compliance with the standards of the Code of Federal Regulations (CFR) part 86, subpart N. The facility is equipped with an engine dynamometer, a dilution tunnel, and a constant volume sampling system. The purpose of the engine dynamometer was to absorb and supply loads from and to the engine. The dynamometer was equipped with a digital encoder to measure the engine speed and the engine torque was measured by employing a load cell. The purpose of the dilution tunnel was to dilute raw emissions with ambient air, to simulate the dilution of the tail pipe exhaust in the atmosphere. Constant volume sampling ensures that each analyzer receives the same volume of gas to assess [57]. A schematic of the EERC test setup is shown in Figure 3.4.1 [57]. 


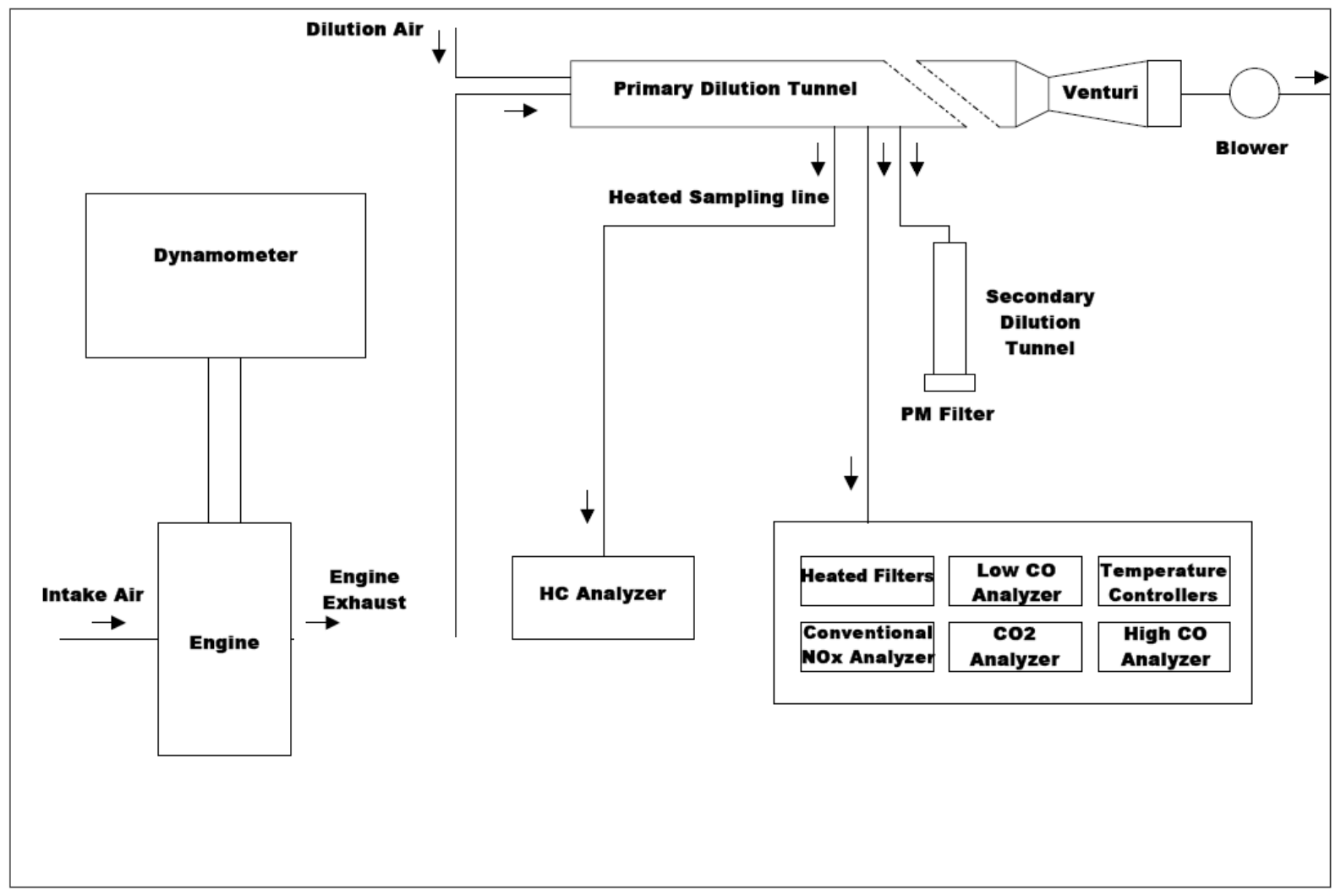

Figure 3.4.1: Schematic of Test Setup at EERC [57]

Initially data obtained by examining the Federal Test Procedure (FTP) cycle was employed as training data for the emissions module of the ANN. The FTP cycle is made up of four portions, the New York Non Freeway (NYNF), the Los Angeles Non Freeway (LANF), Los Angeles Freeway (LAFY), and the forth portion repeats the NYNF portion. The NYNF portion mimics light urban traffic by incorporating frequent starts and stops, the LANF portion models crowded urban traffic with limited stops, and the LAFY portion mimics a crowded expressway in Los Angeles [10]. The FTP cycle is used during certification to verify that newly produced engines meet the current EPA regulations.

\subsubsection{FTP Data}

The Federal Test Procedure cycle that was employed by the engine dynamometer at the WVU EERL to test the 1995 Manufacturer A $400 \mathrm{hp}$ engine is shown in Figures 3.4.1.1 and 3.4.1.2. It is shown that the engine speed does not exceed 2000 RPM, and the maximum torque experienced by the engine does not exceed $1500 \mathrm{ft}$ lbs. 


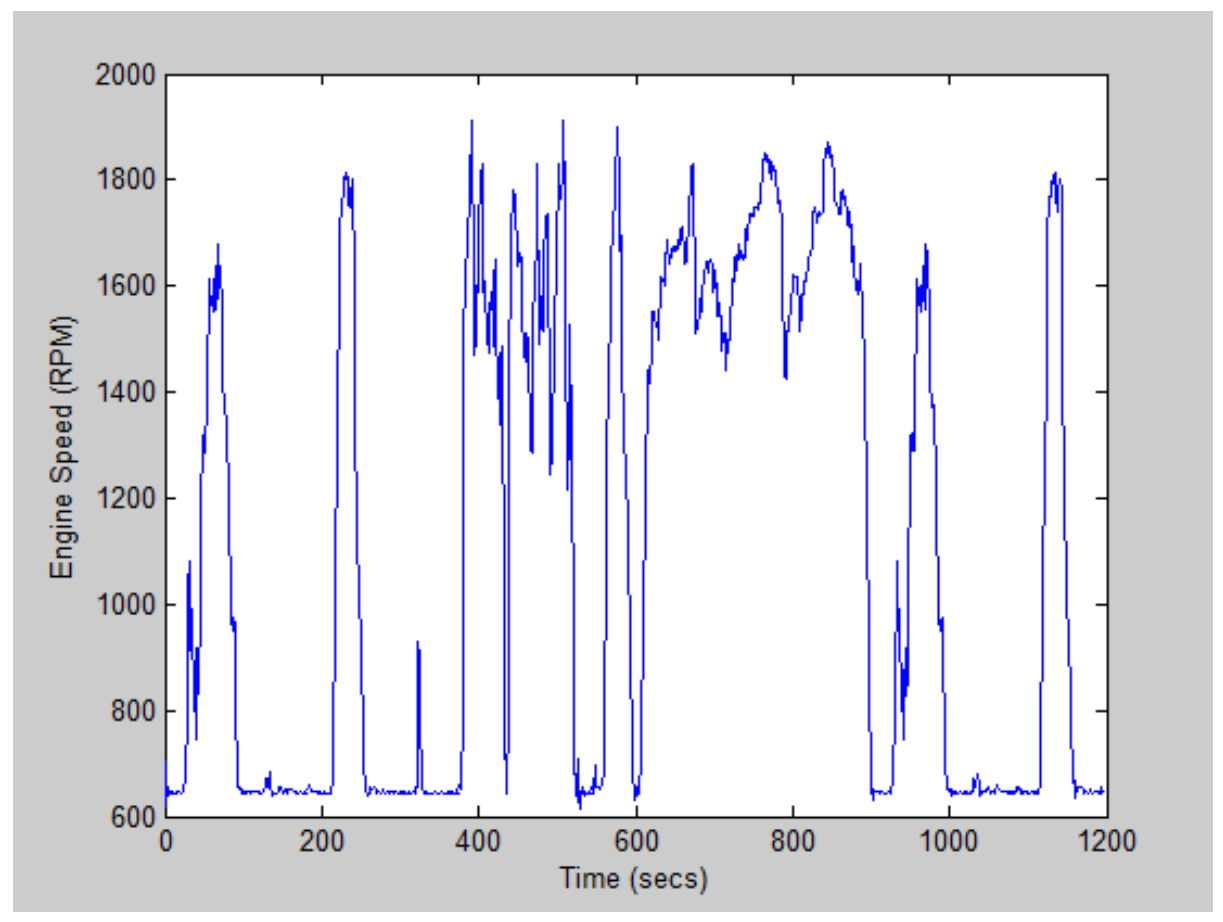

Figure 3.4.1.1: Manufacturer A Engine Speed During a FTP Test

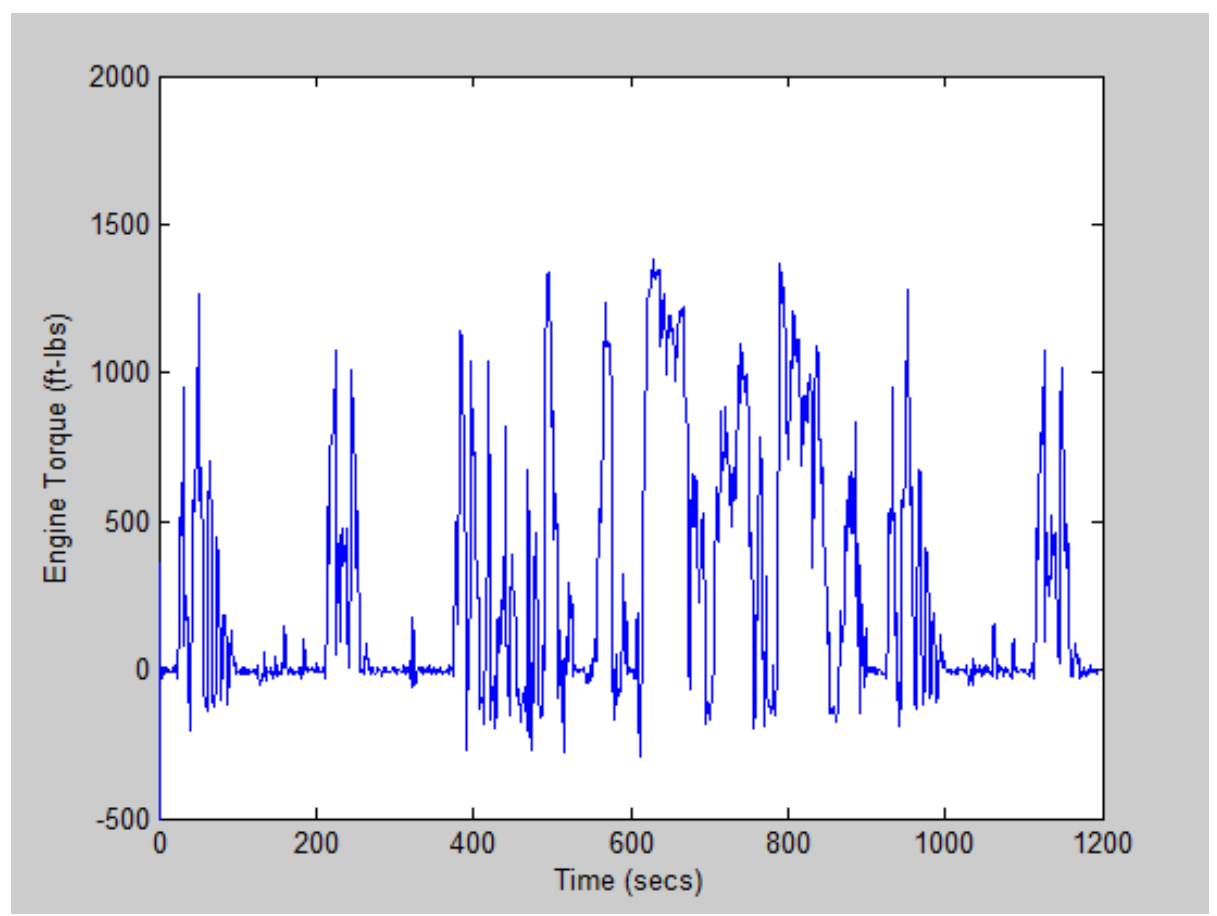

Figure 3.4.1.2: Manufacturer A Engine Torque During a FTP Test 
When data acquired during FTP cycle testing was used to train the emissions module of the ANN, the ANN was determined to under predict the quantity of NOx, and over predict the quantity of $\mathrm{CO}_{2}$ produced during the on-road route being examined. Figure 3.4.1.3 compares the actual and the predicted NOx values when the ANN was trained with emissions data from an FTP cycle and vehicle data from the Wash PA2 1 data set, and then used to predict emissions associated with the Bruceton Mills, WV to Sabraton, WV route. The comparison of measured to predicted $\mathrm{CO}_{2}$ for the training and verification scenario discussed above is shown in Figure 3.4.1.4. The figures show that the trends of the NOx emissions produced along the duration of the route are closely modeled, however the magnitude of the NOx emissions predicted was in some cases half of the measured values.

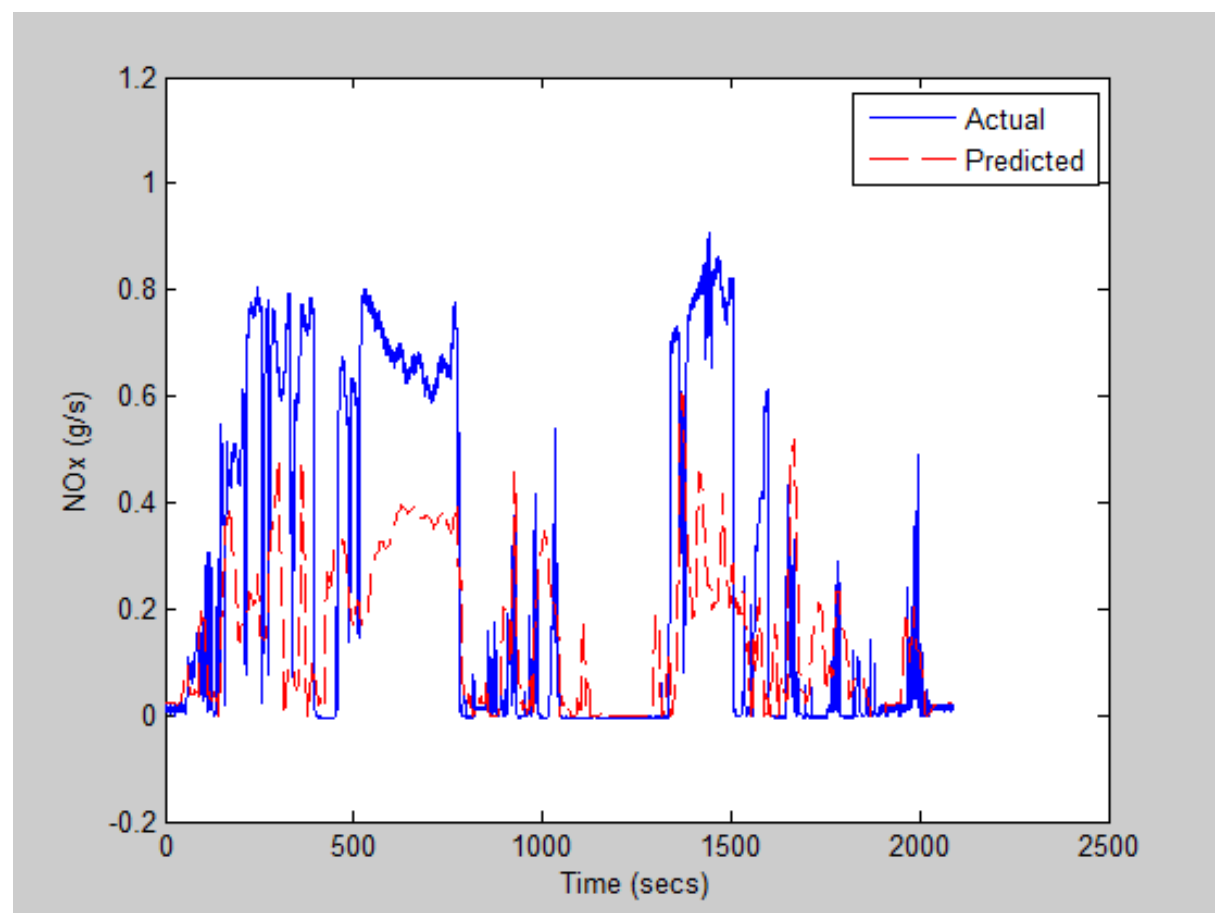

Figure 3.4.1.3: NOx Comparison When Emissions Module was Trained with FTP Data for Bruceton2Sab 2 


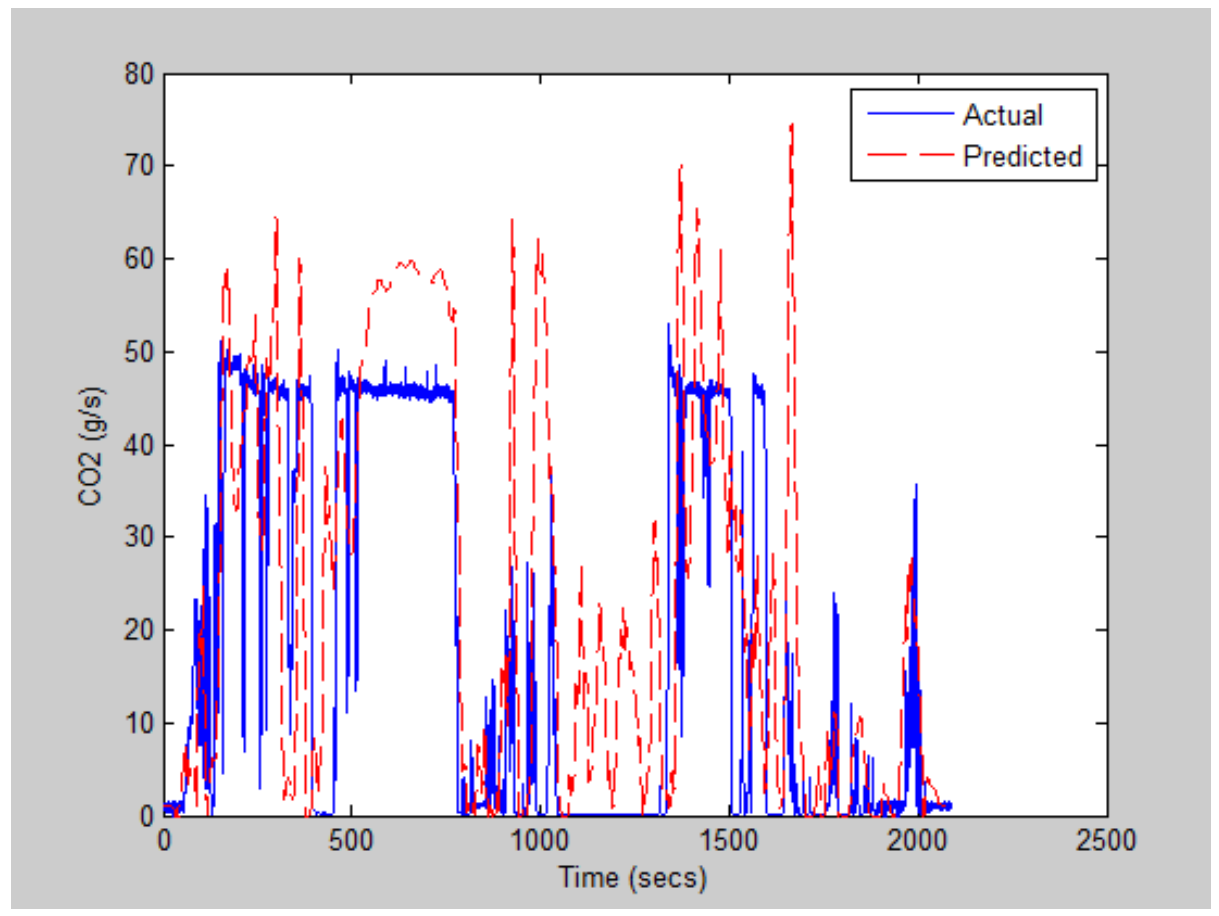

Figure 3.4.1.4: $\mathrm{CO}_{2}$ Comparison When Emissions Module was Trained with FTP Data for Bruceton2Sab2

Due to the model year of the engine, it was determined that a defeat device could have been incorporated into the Manufacturer A $400 \mathrm{hp}$ engine ECU. The defeat device would recognize the FTP cycle and alter the injection timing in order to meet the EPA standards, meaning the engine would operate differently in-use and produce different emissions. In order to determine if the FTP data obtained from testing the Manufacturer A $400 \mathrm{hp}$ engine was the result of a defeat device, data from a different engine dynamometer cycle was examined.

An engine dynamometer test cycle had previously been developed to simulate a vehicle traveling on the Bruceton Mills, WV route, and data was available from testing of the 1995 Manufacturer A $400 \mathrm{hp}$ engine on this cycle. Figure 3.4.1.5 shows the NOx emissions produced as a function of power. It is shown that when the Bruceton Mills, WV cycle was compared to the FTP cycle, the NOx emissions were nearly $40 \%$ higher at the maximum horsepower. Also, at various other powers the NOx emissions were higher than those measured during the FTP cycle. This comparison showed that the engine ECU was operating differently during the FTP cycle than the Bruceton Mills, WV cycle. It was determined that data obtained from FTP cycle testing for model years prior to the consent decrees may not accurately represent the in-use emissions. Due 
to the inability of the FTP cycle data to accurately predict in-use emissions, it was determined that other engine dynamometer cycles should be examined for training the ANN.

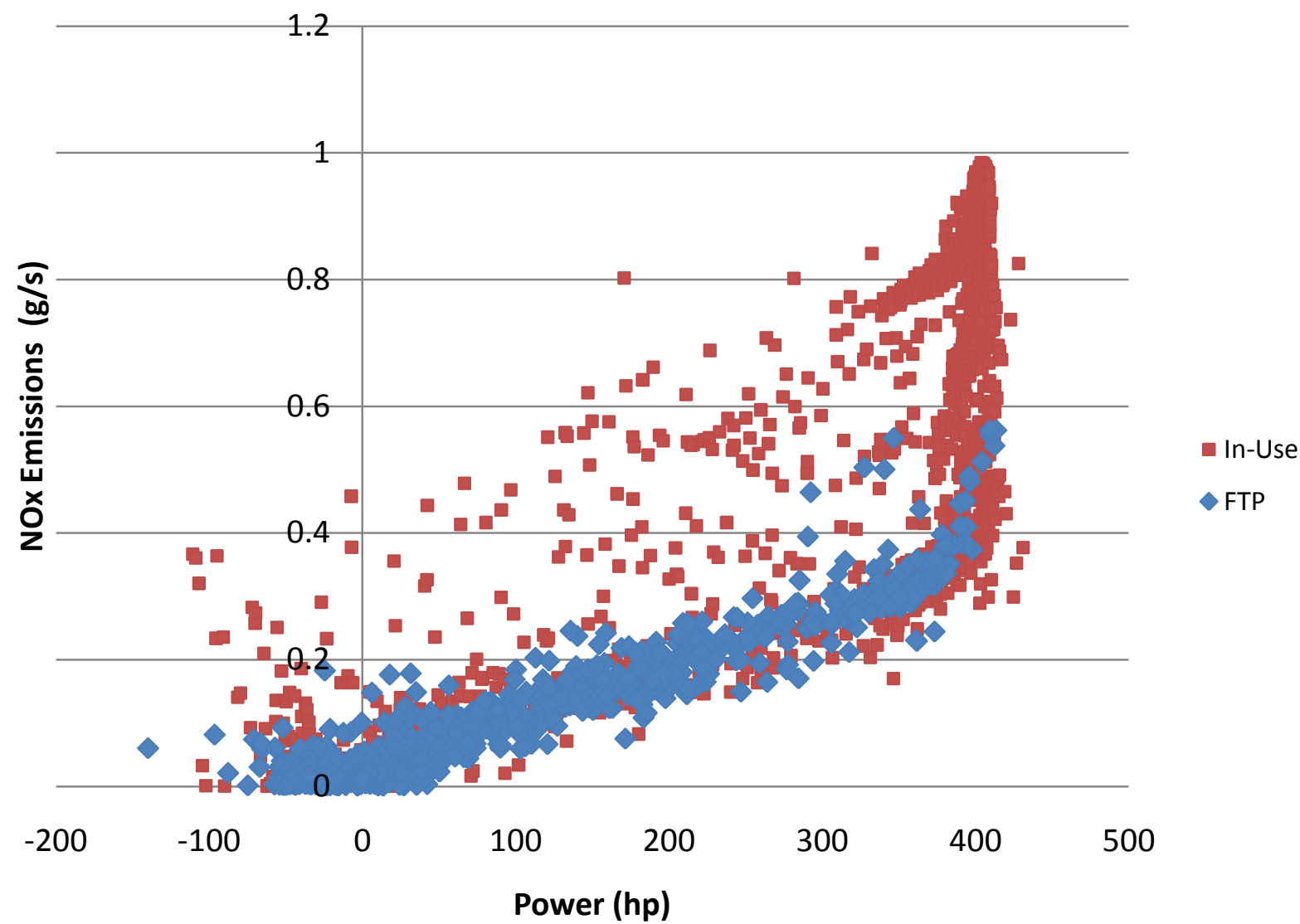

Figure 3.4.1.5: NOx Emissions versus Power for Bruceton Mills, WV and FTP Engine Dynamometer Data

\subsubsection{Bruceton Mills, WV Cycle}

In addition to the Federal Test Procedure Cycle, three other cycles had been generated by previous research efforts and were employed in the research discussed in this dissertation. A cycle was developed to simulate the route from Bruceton Mills, WV to Sabraton, WV, the engine speed and torque associated with this cycle for the Manufacturer A $400 \mathrm{hp}$ engine are shown in Figure 3.4.2.1 and 3.4.2.2, respectively. 


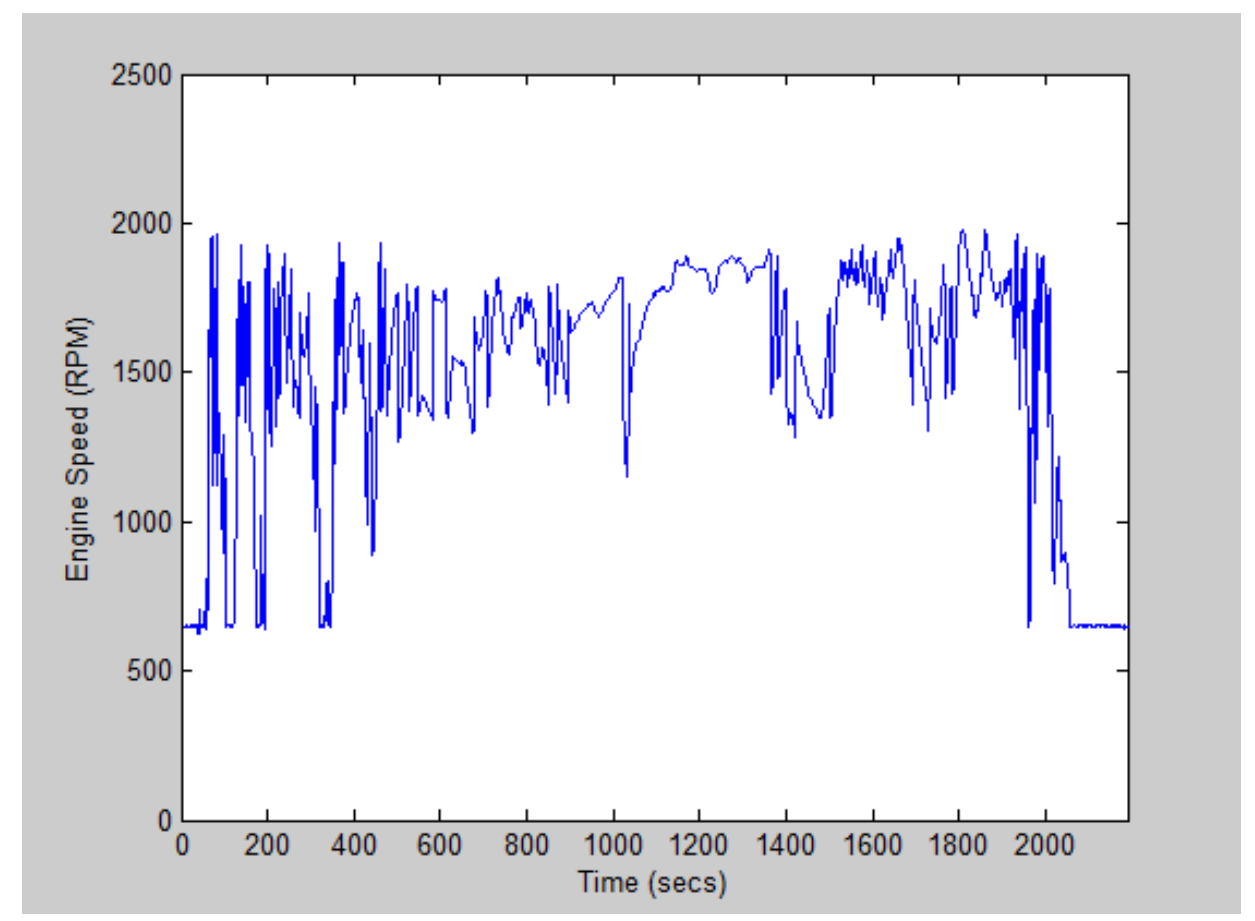

Figure 3.4.2.1 Engine Speed for Sabraton, WV to Bruceton Mills, WV Engine Dynamometer Testing

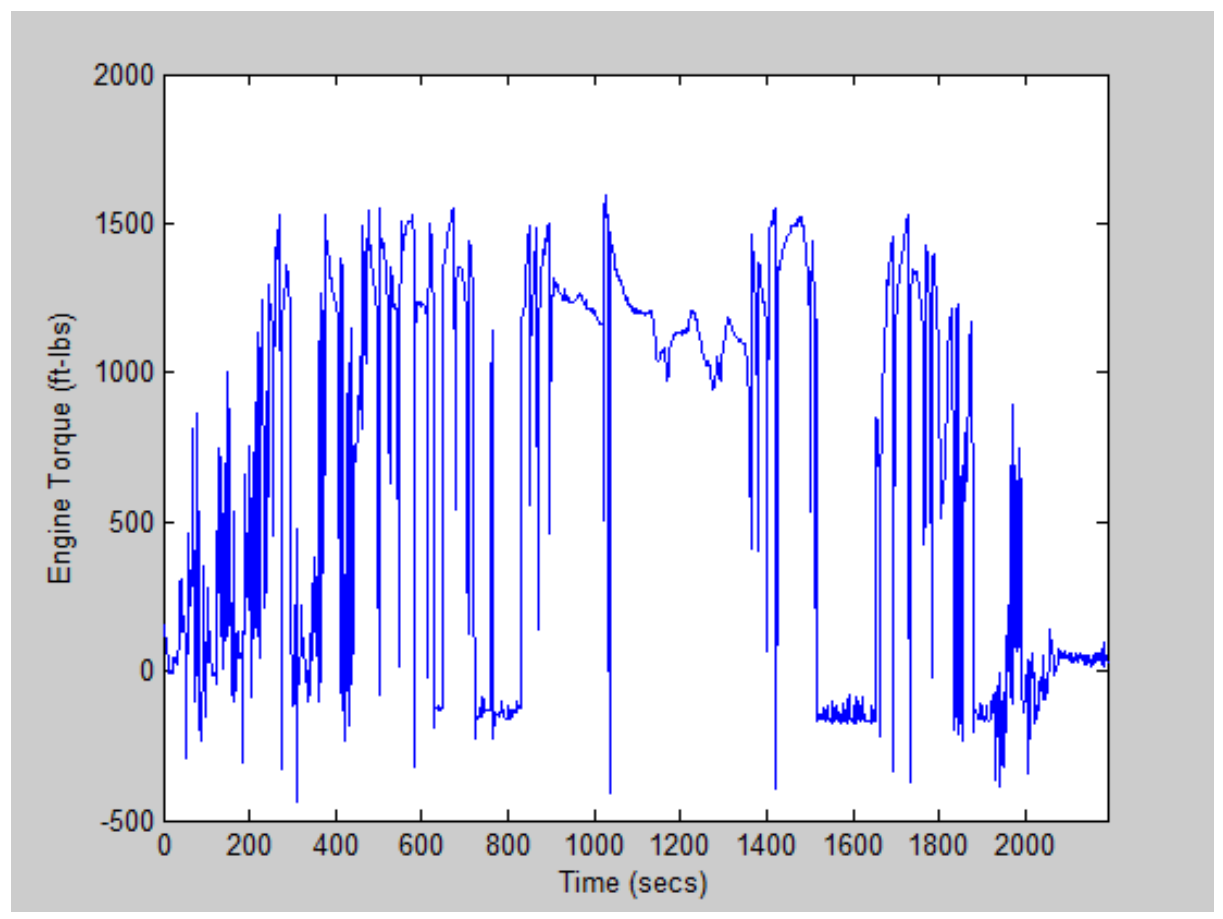

Figure 3.4.2.2: Engine Torque for Sabraton, WV to Bruceton Mills, WV Engine Dynamometer Testing 


\subsubsection{Washington, PA Cycle}

A cycle was developed to simulate the Washington, PA route, for which the engine speed and torque associated with the Manufacturer A $400 \mathrm{hp}$ engine are shown in Figure 3.4.3.1 and 3.4.3.2, respectively.

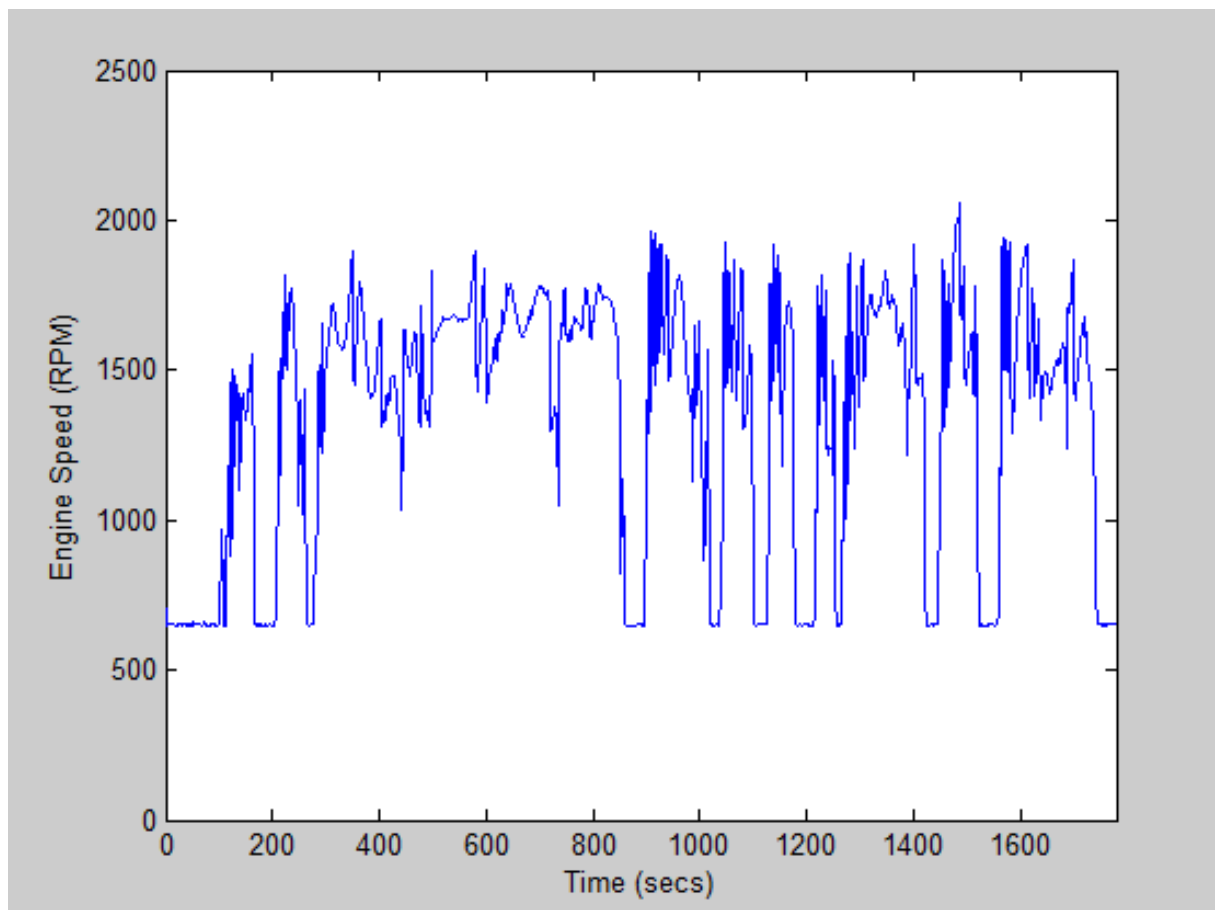

Figure 3.4.3.1: Engine Speed for Washington, PA Engine Dynamometer Testing 


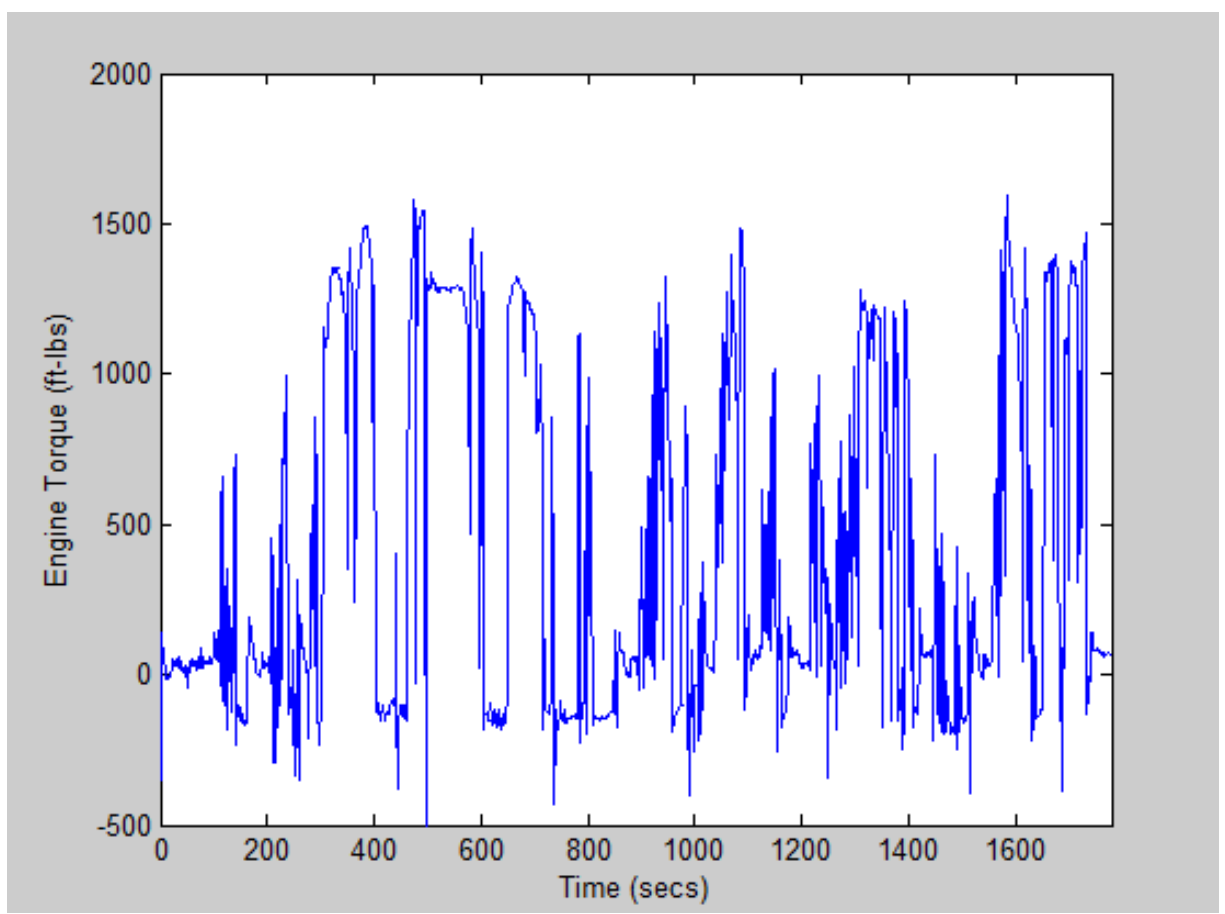

Figure 3.4.3.2: Engine Torque for Washington, PA Engine Dynamometer Testing

Another engine dynamometer cycle was developed to simulate the route from Bruceton Mills, WV to Sabraton, WV. The engine speed and torque associated with this route when the Manufacturer A $400 \mathrm{hp}$ engine was analyzed are show in Figures 3.4.3.3 and 3.4.3.4, respectively. 


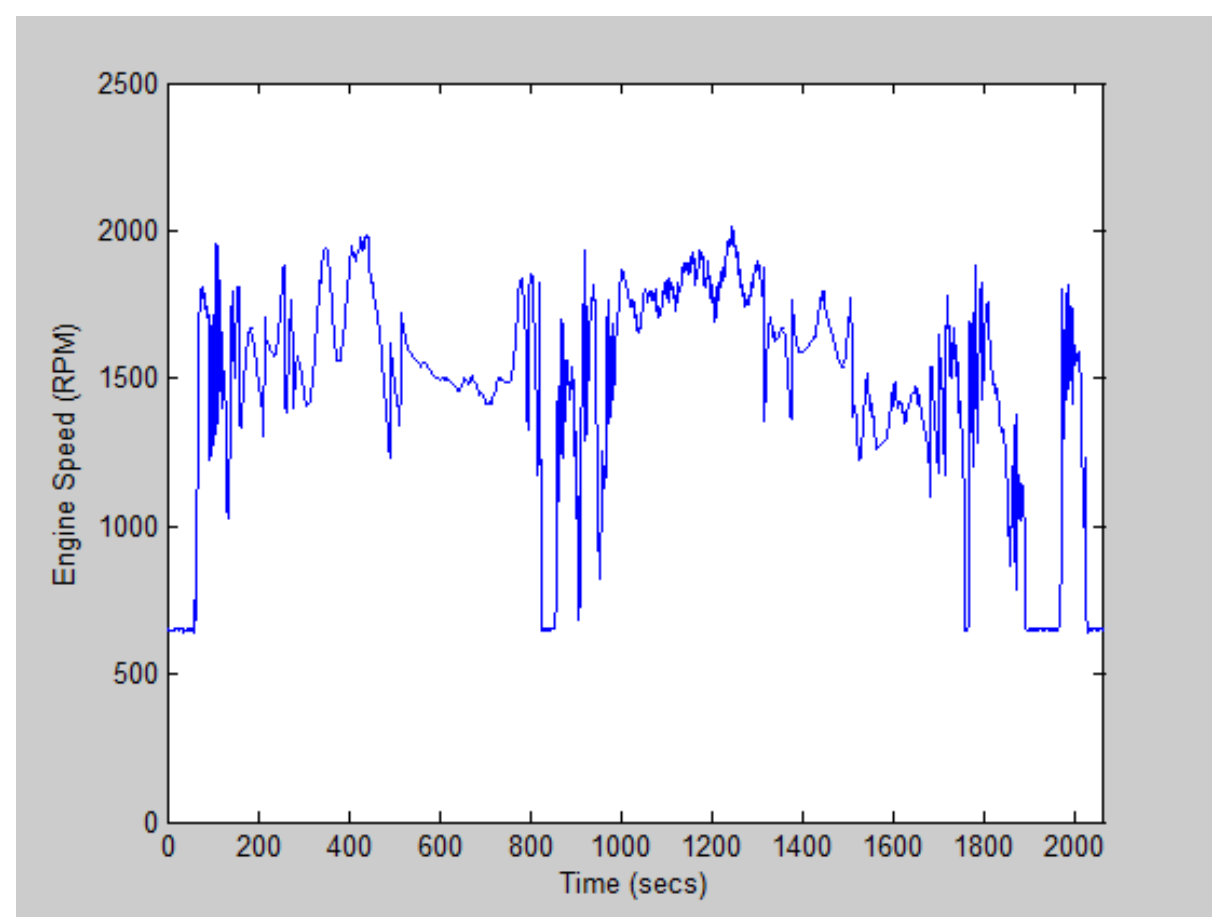

Figure 3.4.3.3: Engine Speed for Bruceton Mills, WV to Sabraton, WV Engine Dynamometer Testing

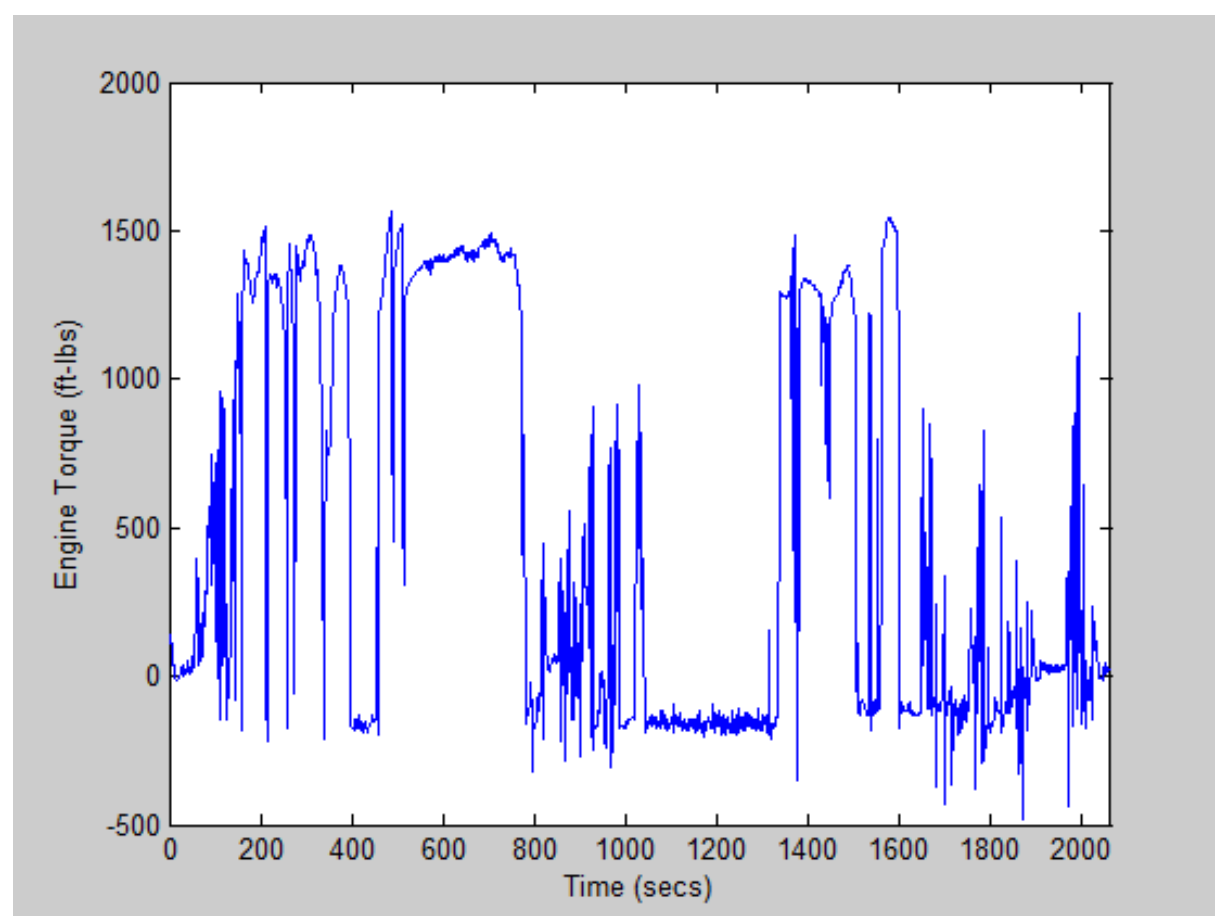

Figure 3.4.3.4: Engine Torque for Bruceton Mills, WV to Sabraton, WV Engine Dynamometer Testing 


\section{Repeatability of Measured Emissions Data}

\subsection{Introduction}

A summary of MEMS data was examined in order to determine the variation in measured emissions values between tests. In order to determine this variation, the integrated emissions values for multiple runs of a particular route were averaged. The standard deviation of the averaged integrated values was determined, and then a coefficient of variation (COV) associated with two standard deviations was calculated. It was important to determine the variation between measured data sets in order to understand the achievable accuracy of the model. Since the ANN model was trained with data that was acquired through in-use testing and the predicted emissions of the model were compared to emissions measured through in-use testing, the determined model accuracy was impacted by the variability in measurement between in-use tests.

\subsection{Repeatability of an Engine}

Table 4.2.1 displays the average integrated values and standard deviations for $\mathrm{NOx}$ and $\mathrm{CO}_{2}$ emissions associated with a 1995 Manufacturer A $400 \mathrm{hp}$ engine. The test weights for the vehicle equipped with this engine ranged from 78,320 lbs to 78,480 lbs. The COV represents the variation between the measured emissions values of runs on the same route, which occurred at different times. Each route was run three times. It was determined that for the Manufacturer A $400 \mathrm{hp}$ engine traveling the Washington, PA route a COV of up to $11.1 \%$ occurred. In order to obtain the average values displayed in the table, multiple data sets from the same route were evaluated to determine the integrated emissions values, and then the average of these integrated emission values was calculated. The standard deviation of the average integrated values was also determined. 
Table 4.2.1 Statistical Analysis of MEMS Data Summary for Manufacturer A 400 hp Engine

\begin{tabular}{|c|c|c|c|c|c|c|}
\hline Route & $\begin{array}{c}\text { Average of } \\
\text { Integrated } \\
\mathrm{CO}_{2} \\
\text { (g/bhp-hr) }\end{array}$ & $\begin{array}{c}\text { Standard } \\
\text { Deviation } \\
\text { of } \\
\text { Integrated } \\
\mathrm{CO}_{2} \\
\text { (g/bhp-hr) }\end{array}$ & $\begin{array}{c}\text { COV } \\
\text { (\%) }\end{array}$ & $\begin{array}{c}\text { Average of } \\
\text { Integrated } \\
\text { NOx } \\
\text { (g/bhp-hr) }\end{array}$ & $\begin{array}{c}\text { Standard } \\
\text { Deviation } \\
\text { of } \\
\text { Integrated } \\
\text { NOx } \\
\text { (g/bhp-hr) }\end{array}$ & $\begin{array}{r}C O V \\
(\%)\end{array}$ \\
\hline $\begin{array}{c}\text { Bruceton Mills to } \\
\text { Sabraton }\end{array}$ & 425.3 & 4.45 & 2.1 & 6.02 & 0.14 & 4.8 \\
\hline $\begin{array}{l}\text { Sabraton to } \\
\text { Bruceton Mills }\end{array}$ & 444.4 & 10.20 & 4.6 & 6.35 & 0.20 & 6.4 \\
\hline Washington PA 1 & 424.1 & 4.55 & 2.1 & 5.32 & 0.21 & 8.0 \\
\hline Washington PA 2 & 426.5 & 7.16 & 3.4 & 5.13 & 0.28 & 11.1 \\
\hline Washington PA 3 & 405.1 & 1.02 & 0.5 & 6.08 & 0.10 & 3.4 \\
\hline Average & 425.1 & 5.48 & 2.6 & 5.78 & 0.19 & 6.6 \\
\hline
\end{tabular}

Table 4.2.2 displays the average integrated values and standard deviations of the integrated values for an engine from Manufacturer $\mathrm{D}$. The average standard deviation of measured $\mathrm{CO}_{2}$ for the five routes considered was 28.39. The $\mathrm{COV}$ for the measured $\mathrm{CO}_{2}$ values ranged from $3.1 \%$ to $33.1 \%$, while the COV values for the measured NOx ranged from $14 \%$ to $75 \%$. The statistics associated with the Washington, PA 3 run were examined further due to the significant difference between the standard deviations associated with it and other runs. It was determined that two data sets were averaged, and compared for the Washington, PA 3 route. The average inferred torque achieved on the two runs differed by $203.14 \mathrm{ft}-\mathrm{lbs}$, between $700.28 \mathrm{ft}-\mathrm{lbs}$ and $497.14 \mathrm{ft}-\mathrm{lbs}$. The average power also differed between the two runs by over $50 \mathrm{hp}$. These differences in torque and power account for the differences in emissions between the two runs. It was determined that a different driver drove each of the runs of the Washington, PA 3 route, therefore driver behavior could be responsible for the differences in torque and power. Also, the ambient temperature during testing reached $84^{\circ} \mathrm{F}$ during one run, while an ambient temperature of only $63^{\circ} \mathrm{F}$ was experienced during the second run. During the run that occurred at a higher temperature, a higher average power was recorded, and can be accounted for by auxiliary devices such as the radiator fan or air-conditioning system. 
Table 4.2.2 Statistical Analysis of MEMS Data for Manufacturer D Engine

\begin{tabular}{|c|c|c|c|c|c|c|}
\hline Route & $\begin{array}{c}\text { Average of } \\
\text { Integrated } \mathrm{CO}_{2} \\
\text { (g/bhp-hr) }\end{array}$ & $\begin{array}{c}\text { Standard } \\
\text { Deviation of } \\
\text { Integrated } \\
\mathrm{CO}_{2}(\mathrm{~g} / \mathrm{bhp}-\mathrm{hr})\end{array}$ & $\begin{array}{l}\text { COV } \\
(\%)\end{array}$ & $\begin{array}{c}\text { Average of } \\
\text { Integrated } \\
\text { NOx } \\
\text { (g/bhp-hr) }\end{array}$ & $\begin{array}{c}\text { Standard } \\
\text { Deviation of } \\
\text { Integrated NOx } \\
\text { (g/bhp-hr) }\end{array}$ & $\begin{array}{l}\text { COV } \\
\text { (\%) }\end{array}$ \\
\hline $\begin{array}{l}\text { Bruceton Mills to } \\
\text { Sabraton }\end{array}$ & 499.6 & 18.64 & 7.5 & 5.08 & 0.49 & 19.3 \\
\hline $\begin{array}{c}\text { Sabraton to } \\
\text { Bruceton Mills }\end{array}$ & 477.6 & 7.44 & 3.1 & 4.95 & 0.34 & 13.8 \\
\hline Washington PA 1 & 507.5 & 17.29 & 6.8 & 5.14 & 0.79 & 30.6 \\
\hline Washington PA 2 & 501.1 & 13.12 & 5.2 & 5.34 & 0.89 & 33.5 \\
\hline Washington PA 3 & 516.1 & 85.45 & 33.1 & 6.52 & 2.46 & 75.4 \\
\hline Average & 500.4 & 28.39 & 11.4 & 5.41 & 0.99 & 36.8 \\
\hline
\end{tabular}

Table 4.2.3 displays the average values for average integrated emissions, the standard deviation of integrated emissions, and the COV for each emission constituent measured. These average values were calculated by considering all engines and all routes that were examined with the MEMS. It was determined that the average $\mathrm{COV}$ for the integrated measured $\mathrm{CO}_{2}$ values was $2.5 \%$, and the average COV for the integrated measured NOx values was $4.8 \%$.

Table 4.2.3: Statistical Analysis of MEMS Data For All Engines

\begin{tabular}{|c|c|c|c|c|c|c|}
\hline $\begin{array}{c}\text { All Routes and All } \\
\text { Engines }\end{array}$ & $\begin{array}{c}\text { Average of } \\
\text { Integrated } \\
\mathrm{CO}_{2}(g / b h p-h r)\end{array}$ & $\begin{array}{c}\text { Standard } \\
\text { Deviation } \\
\text { of } \\
\text { Integrated } \\
\mathrm{CO}_{2} \\
\text { (g/bhp-hr) }\end{array}$ & $\begin{array}{c}\text { COV } \\
(\%)\end{array}$ & $\begin{array}{c}\text { Average of } \\
\text { Integrated } \\
\text { NOx } \\
\text { (g/bhp-hr) }\end{array}$ & $\begin{array}{c}\text { Standard } \\
\text { Deviation of } \\
\text { Integrated } \\
\text { NOx } \\
\text { (g/bhp-hr) }\end{array}$ & $\begin{array}{c}\text { COV } \\
\text { (\%) }\end{array}$ \\
\hline Average & 483.0 & 11.93 & 4.9 & 6.05 & 0.28 & 4.8 \\
\hline
\end{tabular}

The data presented in the above tables demonstrates the variation between emissions data measured during different testing periods on the same route. This difference could be due to various factors such as ambient weather conditions, traffic conditions, and different driving styles. The emissions testing statistics for these two engines were selected for discussion because the Manufacturer A $400 \mathrm{hp}$ engine represented an engine with relatively low variance from test to test, while the engine from Manufacturer D represented an engine with relatively high variance from test to test. 


\subsection{Repeatability of A Vehicle}

Table 4.3.1 displays the average integrated emissions, the standard deviation of the average integrated emissions, and the COV of the average integrated emissions for the similar vehicle engine combinations. This COV depicts the variation in emissions from different engines of the same model, employed in the same type of vehicle. Three 2001 trucks of the same manufacturer, each equipped with a 2001 Manufacturer B, 525 hp, engine were tested multiple times, at different test weights, on the various routes discussed earlier in this document. The data for the statistical analysis performed in the table below was measured during testing on the Sabraton, WV to Bruceton Mills, WV route. It should be noted that these engines are the same year and the same model, with differing serial numbers. The test weights were divided into two bins, weight A includes vehicles tested with weights ranging from 75,000 lbs to $81000 \mathrm{lbs}$. Vehicles ranging in tested weights from 55,000 lbs to $63,000 \mathrm{lbs}$ were designated with the test weight $\mathrm{B}$. The average values of integrated $\mathrm{CO}_{2}$ and integrated $\mathrm{NOx}$ emissions were calculated, as well as the standard deviation of the averages of each engine, for both test weights. It was determined that different engines of the same model and year, traveling the same route, can vary up to $7.2 \%$ in their measured emissions.

Table 4.3.1: Comparison of Different Engines of the Same Engine Model on the Same Route

\begin{tabular}{|c|c|c|c|c|c|}
\hline \multicolumn{6}{|c|}{ Sabraton to Washington } \\
\hline \multicolumn{6}{|c|}{ Year: 2001 Engine Model: Manufacturer B 525 hp (3 Serial Numbers) } \\
\hline $\begin{array}{c}\text { Average of Integrated } \\
\mathrm{CO}_{2}(\mathrm{~g} / \mathrm{bhp}-\mathrm{hr})\end{array}$ & $\begin{array}{c}\text { Standard } \\
\text { Deviation of } \\
\text { Average } \\
\text { Integrated } \\
\mathrm{CO}_{2}(\mathrm{~g} / \mathrm{bhp}-\mathrm{hr})\end{array}$ & $\begin{array}{c}\text { Average } \\
\text { Integrated } \\
\mathrm{CO}_{2} \\
\operatorname{COV}(\%) \\
\end{array}$ & $\begin{array}{c}\text { Average of } \\
\text { Integrated } \\
\text { NOx } \\
\text { (g/bhp-hr) }\end{array}$ & $\begin{array}{c}\text { Standard } \\
\text { Deviation of } \\
\text { Average } \\
\text { Integrated } \\
\text { NOx (g/bhp- } \\
\text { hr) }\end{array}$ & $\begin{array}{c}\text { Average } \\
\text { Integrated } \\
\text { NOx } \\
\operatorname{cov}(\%)\end{array}$ \\
\hline \multicolumn{6}{|c|}{ Weight A } \\
\hline 545.58 & 8.58 & 3.2 & 5.89 & 0.01 & 0.4 \\
\hline \multicolumn{6}{|c|}{ Weight B } \\
\hline 532.09 & 19.15 & 7.2 & 5.91 & 0.43 & 14.7 \\
\hline
\end{tabular}

\subsection{Summary of Repeatability}

Both the repeatability of a route and the repeatability of an engine were examined. Through statistical analysis of the in-use measured $\mathrm{CO}_{2}$ data it was determined that an average COV of $2.6 \%$ existed, when all of the routes that were examined with the $400 \mathrm{hp}$ Manufacturer A engine 
were averaged. The $\mathrm{COV}$ for $\mathrm{CO}_{2}$ emissions for each route ranged from $0.5 \%$ for the Washington, PA 3 route to $4.6 \%$ for the Sabraton, WV to Bruceton Mills, WV route. This means that if the same vehicle and engine setup traveled from Sabraton, WV to Bruceton Mills, WV twice, the measured emissions for each trip could vary by $4.6 \%$. The variation in measured NOx emissions was greater than the $\mathrm{COV}$ associated with $\mathrm{CO}_{2}$, ranging from $3.4 \%$ to $11.1 \%$ for the routes examined with the $400 \mathrm{hp}$ Manufacturer A engine.

It was observed that the $\mathrm{COV}$ of both measured $\mathrm{NOx}$ and $\mathrm{CO}_{2}$ emissions was greater for the lower weight classification when multiple vehicles with the same engine were compared. The average COV between the vehicles for NOx emissions was $14.7 \%$.

The purpose of this statistical analysis was to determine what an achievable accuracy of the ANN model should be. It has been shown from run to run of the same route, and between engine to engine, variations of over $10 \%$ have occurred. Since the ANN was trained with in-use data from the data sets examined above, and the predicted emissions were compared to those data sets that the ANN accuracy could not be expected to be better than variations between measured data sets.

It should also be noted that research conducted by Thompson et al. has shown that employing different fuels during heavy duty diesel emissions testing has resulted in variations in $\mathrm{CO}$ and hydrocarbon production of $40 \%$ and $17 \%$, respectively. The quantity of NOx produced has been shown to vary up to $12 \%$ when fuels obtained from different commercial suppliers were used [71]. 


\section{Model Development}

\subsection{Overview}

The model architecture consisted of multiple modules. The first module employed road grade and vehicle speed to predict the speed and torque experienced by the engine during the route. The second module modeled the emissions produced by the engine, and used the engine speed and torque that were outputs of the first module as inputs. Once the emissions were predicted by the engine model module, a third module of the model would be incorporated to correct the emissions for factors such as deterioration and ambient conditions.

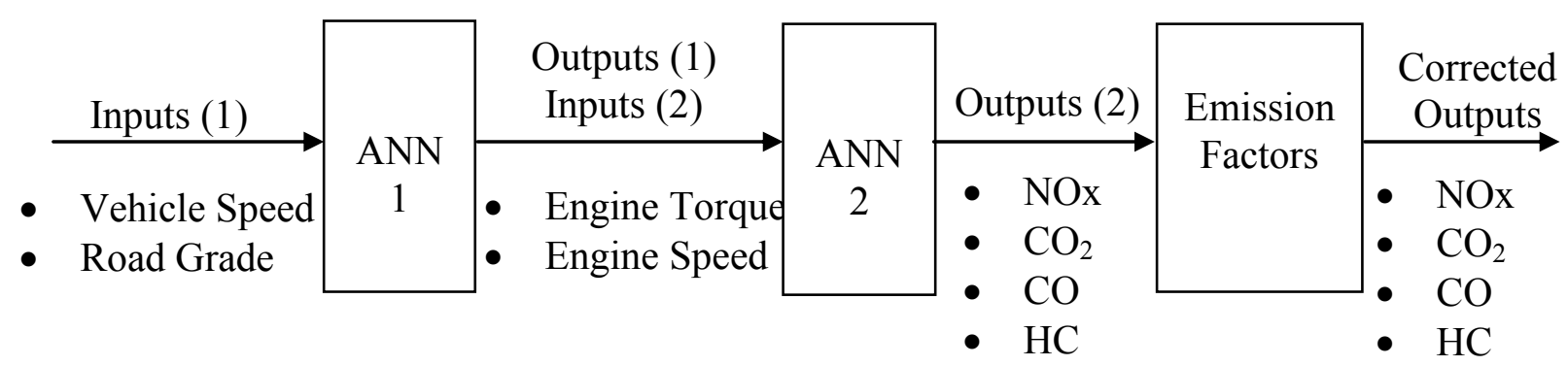

Figure 5.2.1: Basic Structure of Model

\subsection{Model Architecture}

The ANN model developed for this research consisted of two neural networks. One network predicted the engine speed and torque associated with the road grade and vehicle speed of the route being examined, while the other network predicted the emissions associated with the predicted engine speed and torque. In the figure above, ANN 1 represents the engine operation model and ANN 2 represents the emissions prediction model. It is shown that the outputs from the first neural network serve as inputs to the second neural network.

\subsection{Road grade}

Road grade is an input for the ANN, and therefore must either be a known value or calculated. During the training process road grade was calculated for the particular routes being employed. The atmospheric pressure is a function of elevation, therefore from the measured pressure data, an elevation was calculated, and related to that, a change in elevation with time, resulting in the 
traveled road grade. The pressure was measured by the ambient pressure sensor on the MEMS. The equation used to determine the altitude change as a function of the measured ambient pressure is shown as Equation 5.3.1.

$$
h=\left(\frac{T_{b}}{\left(\frac{P}{P_{b}}\right)\left(\frac{R * L_{b}}{g * M}\right)}\right)-\left(\frac{T_{b}}{L_{b}}\right)+h_{b}
$$

In the above equation $T_{b}$ represents the standard temperature, $\mathrm{R}$ is the universal gas constant for air, $\mathrm{L}_{\mathrm{b}}$ is the standard temperature lapse rate, $\mathrm{M}$ is the molar mass of air, $\mathrm{P}_{\mathrm{b}}$ is the standard pressure, g represents gravitational acceleration, and $h_{b}$ is the initial height. The assumption was made that the vehicles would not travel above an altitude of 11,000 $\mathrm{m}$, therefore the standard conditions for elevations less than 11,000 meters were used. The standard temperature was 288.15 K, 29.92 in $\mathrm{Hg}$ was used as the standard pressure, and a temperature lapse of -0.0065 $\mathrm{K} / \mathrm{m}$ was used [61]. The temperature lapse rate accounts for the reduction of temperature associated with elevation increase.

The geometric relationship between the distance traveled and the change in altitude is shown in Figure 5.3.1. The hypotenuse of the triangle represents the actual traveled distance, while $\mathrm{h}$ is the change in elevation which is calculated based on the measured pressure. The traveled distance is determined by employing Equation 5.3.2, which is the product of vehicle speed and the time interval. The side of the triangle labeled $\mathrm{d}$ is calculated by the relationship shown in Equation 5.3.3. The road grade is then the slope of the distance traveled, whose calculation is shown as Equation 5.3.4.

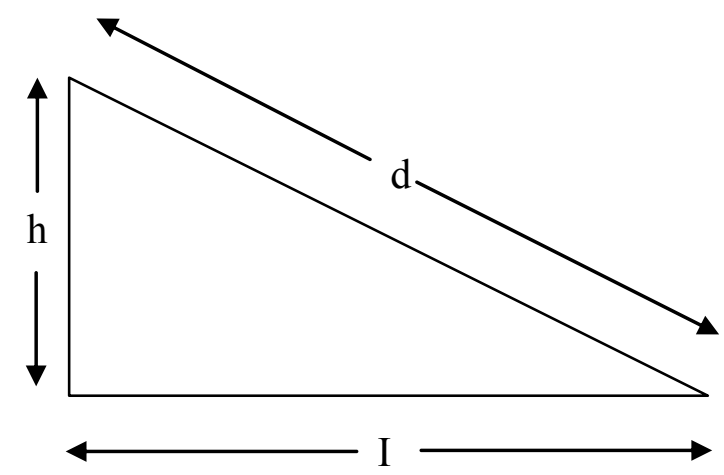

Figure 5.3.1: Diagram of Relationship Between Distance Traveled and Altitude Change 


$$
\mathrm{I}=\sqrt{\mathrm{d}^{2}-\mathrm{h}^{2}}
$$

$$
\mathrm{d}=\text { Vehicle Speed } *(\Delta \mathrm{t})
$$

$$
\text { Road Grade }=100 * \frac{\mathrm{h}_{2}-\mathrm{h}_{1}}{\mathrm{I}}
$$

Figure 5.3.2 shows the calculated height associated with the Bruceton Mills, WV route. The maximum height was less than 650 meters, and the minimum height was 200 meters. Figure 5.3.3 displays the road grade that was calculated as a function of the height. The road grade ranged from $+14 \%$ to $-14 \%$. It should be noted that the height was not found to change relative to the spikes in road grade that occurred at 800 seconds and 1800 seconds, this indicated that the spikes were the results of measurement or other errors in the pressure data.

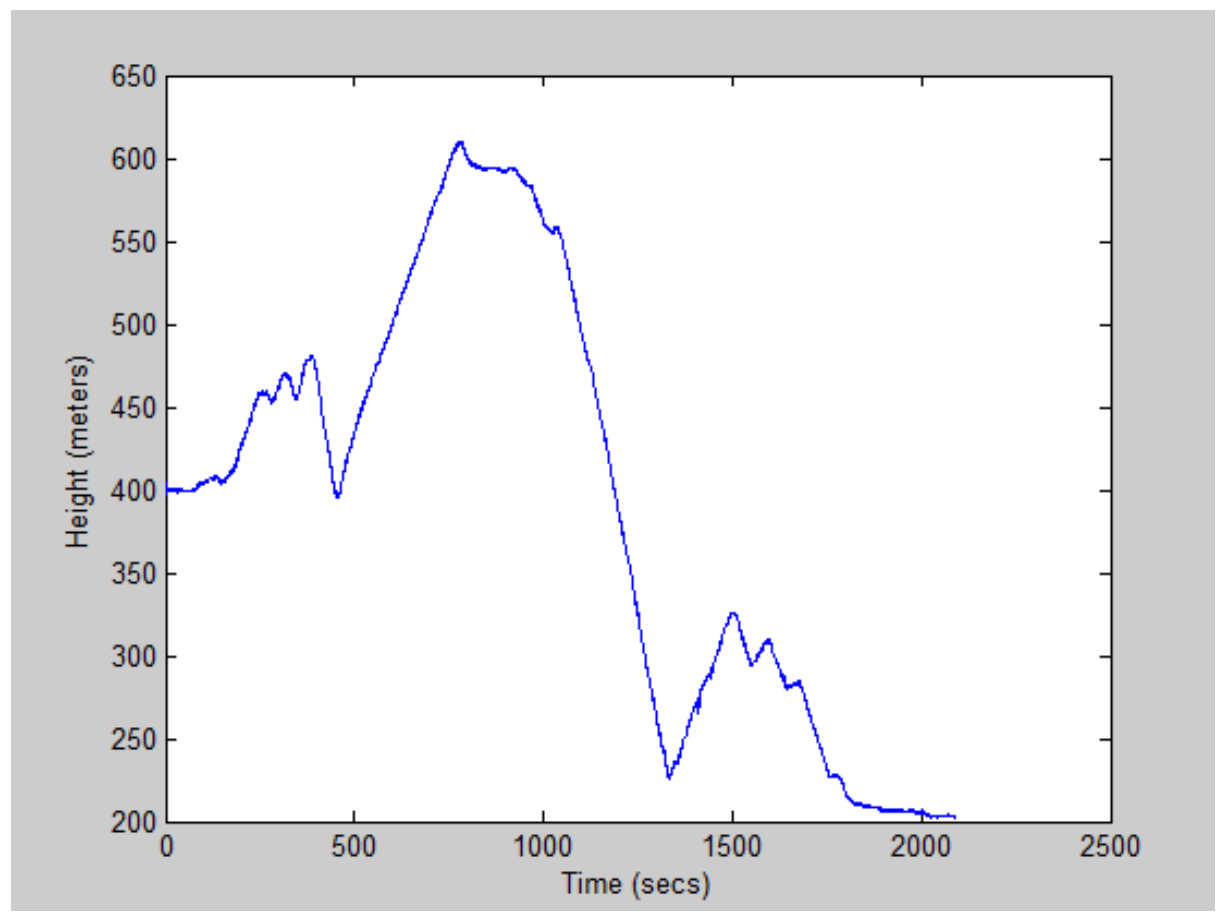

Figure 5.3.2: Height for Bruceton Mills, WV Route 


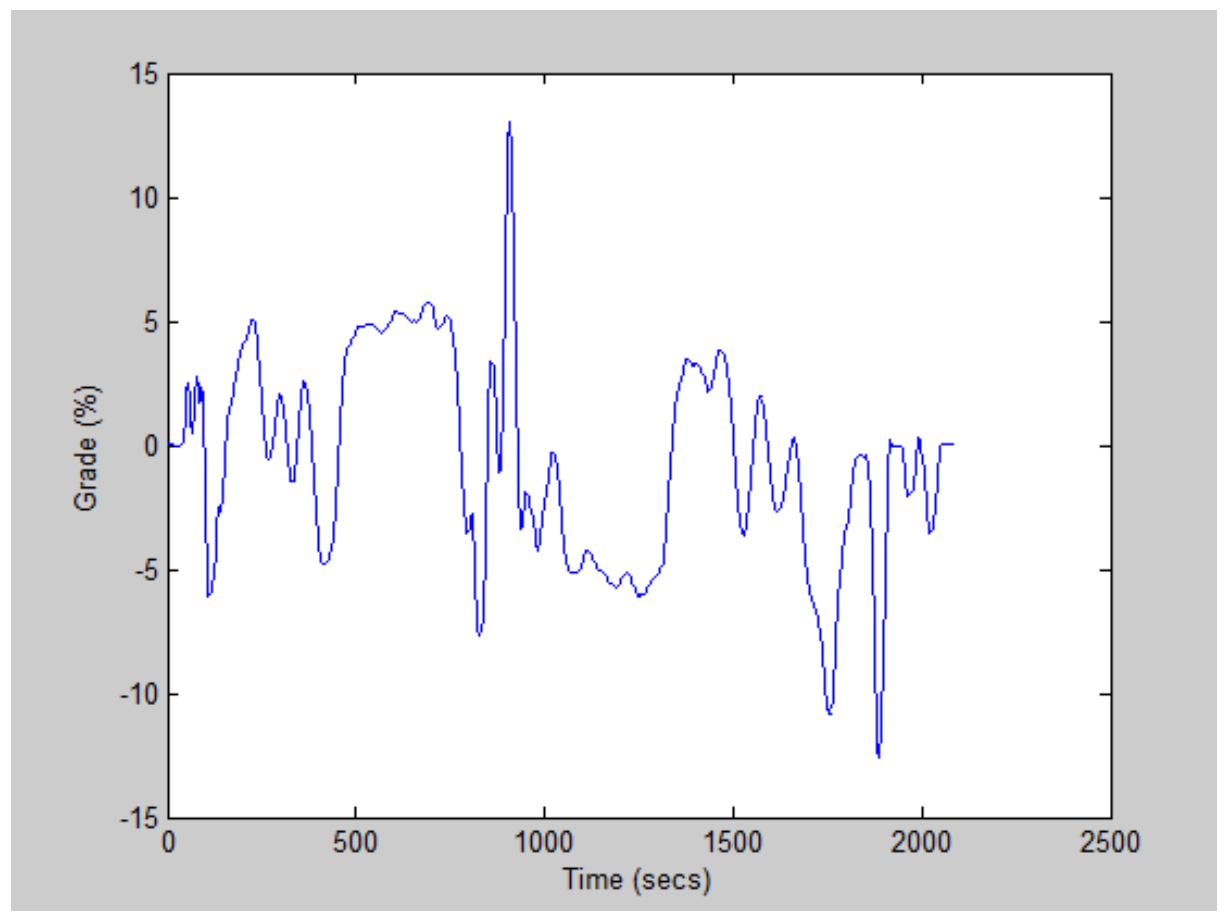

Figure 5.3.3: Road Grade for Bruceton Mills, WV Route

\subsection{Dispersion}

When evaluating engine emissions one must be concerned with the dispersion that occurs between an emission producing engine event and the measured response of the emission constituent analyzers. The engine event is instantaneous, however the output of the analyzers are not, and therefore there is a time alignment issue that arises between the emissions that were actually generated by specific engine operating conditions. It has been shown that amplitude reductions are experienced by the measured responses, this means that measured peak amplitude is less than the amplitude associated with the actual engine event. Various methods of correcting the measured emissions for this dispersion phenomenon have been discussed in the literature. The sequential inversion technique employs the dispersion characteristics of the particular analyzer which was used in testing. In order to use the sequential inversion technique, prior to conducting the emission data acquisition, dispersion characteristics of the analyzer must be determined by injecting $100 \mathrm{ppm}$ of $\mathrm{NO}_{\mathrm{x}}$ in to the dilution tunnel for a 1 second interval. Readings should then be recorded from the analyzer at 1,2,3, and 4 seconds after the injection. The measurements at these time intervals define the dispersion function, which must sum to 
unity. This dispersion function would then be applied to relate the input into the analyzer and the actual output of the analyzer. The following equations are employed in the sequential inversion technique. Equation 5.4.1 represents the dispersion function, where $\mathrm{C}_{1}$ through $\mathrm{C}_{4}$ are the readings of the analyzer after the one second sample injection. In Equation 5.4.2, $\mathrm{j}$ ranges from 1 to the number of seconds of output data considered, and $\mathrm{k}$ denotes the number of terms in the dispersion function. $Y\left(t_{j}\right)$ represents the output of the analyzer and $U\left(t_{j}\right)$ represents the input to the analyzer [60].

$$
\begin{array}{ll}
\left\{C_{1} C_{2} C_{3} C_{4}\right\} & \text { Equation 5.4.1 } \\
Y\left(t_{j}\right)=C_{1} U\left(t_{j}\right)+C_{2} U\left(t_{j-1}\right)+C_{3} U\left(t_{j-2}\right)+\cdots+C_{k} U\left(t_{j-k+1}\right) & \text { Equation 5.4.2 }
\end{array}
$$

Another method of reconstructing the actual transient emission from the measured value is the differential coefficients method. It is assumed in this method that the input to the analyzer can be represented as a linear combination of the first and second order derivatives of the output and the actual output. Equation 5.4.3 shows this linear combination, in this equations $\mathrm{Y}(\mathrm{t})$ denotes the output of the analyzer, and $U(t)$ denotes the input to the analyzer. The differential coefficients method assumes that all data was accounted for by the analyzer, therefore the integrated value of the inputs is equivalent to the integrated value of the outputs.

$$
U(t)=Y(t)+a_{1} Y^{\prime}(t)+a_{2} Y^{\prime \prime}(t)
$$

A dispersion function was generated by exposing the analyzer to a 1 second pulse of input. The unit impulse input and the derivatives were mapped and then the time sequence was fitted over the dispersion period. An error was calculated as the difference between the input to the analyzer and the output, the first derivative of the output multiplied by a constant, and the second derivative multiplied by a constant. The values of these constants were then obtained by calculating the least-squared error at each point, and minimizing this error for the best fit. Once the constants were determined, the input to the analyzer for any output can be determined [56]. 
Another method of compensating for the dispersion associated with emission measurement is to disperse the axle power. This methods results in power values that lack physical significance, however reflect what the axle power would be if it were dispersed through the tunnel similar to the emissions. The equation employed to model the dispersion of power for this method is shown below in Equation 5.4.4. This equation is used to determine $\mathrm{C}_{\mathrm{i}}$, the concentration of the dispersion model at an instant in time. In this equation theta represents the ratio of the one second increment that was centered about the mean time shift between the power peaks to the mean time shift between power peaks. The dimensionless vessel dispersion number is related to the extent of axial dispersion, and is represented by the value $\mathrm{D} / \mathrm{uL}$.

$$
C_{i}=\frac{1}{2 \sqrt{\pi \theta_{i}(D / u L)}} \exp \left[-\frac{\left(1-\theta_{i}\right)^{2}}{4 \theta_{i}(D / u L)}\right]
$$

After determining the $C_{i}$ values, they are normalized, and a curve is generated. This curve is then employed in conjunction with the axle power data to produce the dispersed axle power $[57,59]$. For this research effort was made to backwards transform the measured emissions to what their values would have been at the exhaust manifold. A method was developed that employed Equation 5.4.3 and attempted to fit a polynomial to the function in order to obtain the constants $\mathrm{a}_{1}$ and $\mathrm{a}_{2}$. An attempt was made to align the measured emissions with the measured power in order to facilitate the backwards transformation. After analyzing the results it was determined that this was not an accurate way to backwards transform the emissions and actual analyzer characteristics would be needed to accurately backwards transform the emissions data. Since the data being used to verify this model was acquired over a number of years, and with analyzers that have been serviced and recalibrated, it would not be feasible or accurate to examine the analyzer characteristics and then employ them to backwards transform the measured emissions. The ANN accounts for the dispersion effects by using inputs that have been pre-processed by applying moving averages. The moving average of no fewer than 20 points was calculated at each data point. For the engine dynamometer data this was a moving average over 20 seconds, which will limit the effects of dispersion on the model. The emissions predicted and measured emissions were also compared on an integrated, rather than continuous basis, therefore error due to any dispersion effects were minimized. 


\subsection{Vehicle Weight}

The weight of a vehicle affects the power requirements, and therefore impacts the emissions produced by an engine. The ANN model was trained with engine speed and torque data that were associated with a specific vehicle test weight. In order for the model to be applied to a wider range of in-use scenarios, a correction factor was developed to compensate for a difference in vehicle weight. The correction factor was applied to the outputs of ANN1, prior to the outputs being employed as inputs to ANN2. The correction is applied to the power, which is a function of engine speed and engine torque. It was assumed that the engine speed was constant between the two vehicles of different weights and the torque was scaled based on the following equations. The road load equation is shown as Equation 5.5.1 and was employed to develop one possible method of compensating for weight differences when modeling. In the displayed equation, $\mathrm{P}$ stands for the power required to maintain a steady speed, the density of air is denoted with $\rho, C_{D}$ is the aerodynamic drag coefficient of the specific vehicle, $\mathrm{V}$ is the vehicle velocity, $\mathrm{A}$ is the frontal area of the vehicle, $\mu$ is the rolling resistance coefficient, $m$ is the mass of the vehicle, and the angle of inclination of the road grade is represented by $\theta$ [68].

$$
\mathrm{P}=\frac{1}{2} \rho \mathrm{C}_{\mathrm{D}} A \mathrm{~V}^{3}+\mu \mathrm{gmV}+\mathrm{mgsin}(\theta) \mathrm{V}+\mathrm{maV}
$$

In order to determine a method of correction for applying the ANN to similar vehicle with different weights, assumptions were made. It was assumed that density, velocity, and acceleration were constant. It was also assumed that the vehicles being examined would have the same frontal area, rolling resistance coefficient, and aerodynamic drag coefficient. If the specific values for frontal area, rolling resistance coefficient, and aerodynamic drag coefficient are not available for the actual vehicle being modeled, average values for that type of vehicle could be assumed.

The road load equation should be written for the vehicle at each test weight, as shown in Equations 5.5.2 and 5.5.3. In these equations the subscript 1 represents the weight at which the model was trained, and subscript 2 represents the weight of the vehicle to be modeled. The terms without subscripts are common to both equations. 


$$
\begin{array}{ll}
P_{1}=\frac{1}{2} \rho C_{D} A V^{3}+\mu g m_{1} V+m_{1} g \sin (\theta) V+m_{1} a V & \text { Equation 5.5.2 } \\
P_{2}=\frac{1}{2} \rho C_{D} A V^{3}+\mu g m_{2} V+m_{2} g \sin (\theta) V+m_{2} a V & \text { Equation 5.5.3 }
\end{array}
$$

For ease of calculation the products of the constants in the above equations were relabeled as single constants. Equations 5.5.4 and 5.5.5 show the simplified equations and Equations 5.5.65.5.9 show the constant substitutions.

$$
\begin{aligned}
& \mathrm{P}_{1}=\mathrm{C}_{1}+\mathrm{C}_{2} \mathrm{~m}_{1}+\mathrm{C}_{3} \mathrm{~m}_{1}+\mathrm{C}_{4} \mathrm{~m}_{1} \\
& \mathrm{P}_{2}=\mathrm{C}_{1}+\mathrm{C}_{2} \mathrm{~m}_{2}+\mathrm{C}_{3} \mathrm{~m}_{2}+\mathrm{C}_{4} \mathrm{~m}_{2} \\
& C_{1}=\frac{1}{2} \rho \mathrm{C}_{\mathrm{D}} A V^{3} \\
& \mathrm{C}_{2}=\mu \mathrm{gV} \\
& \mathrm{C}_{3}=\mathrm{g} \sin (\theta) \mathrm{V} \\
& \mathrm{C}_{4}=\mathrm{aV}
\end{aligned}
$$

Equation 5.5.9

The common mass terms were then grouped in Equations 5.5.4 and 5.5.5, and a new constant, $\mathrm{K}$, was defined to represent the sum of the constants $\mathrm{C}_{2}, \mathrm{C}_{3}$, and $\mathrm{C}_{4}$. The simplified versions of these equations are shown as Equations 5.5.10 and 5.5.11.

$$
\begin{aligned}
& \mathrm{P}_{1}=\mathrm{C}_{1}+\mathrm{Km}_{1} \\
& \mathrm{P}_{2}=\mathrm{C}_{1}+\mathrm{Km}_{2}
\end{aligned}
$$

Equation 5.5.10 was then rearranged in order to solve for the constant $\mathrm{K}$, this allowed the substitution for $\mathrm{K}$ into Equation 5.5.11. Equation 5.5.12 shows the resulting equation which determines the power associated with the vehicle being modeled at a different test weight.

$$
\mathrm{P}_{2}=\mathrm{C}_{1}+\mathrm{m}_{2}\left(\frac{\mathrm{P}_{1}-\mathrm{C}_{1}}{\mathrm{~m}_{1}}\right)
$$

Equation 5.5.12

An example of accounting for the weight of the vehicle is shown below. For this example a drag coefficient of 0.69 was used, and it was assumed that the frontal area of the vehicle was $115.2 \mathrm{ft}^{2}$. 
The density of air was assumed to be constant at $3.373 \times 10^{-3} \mathrm{slugs} / \mathrm{ft}^{3}$, which is the density of air at standard pressure and $60^{\circ} \mathrm{F}$. This temperature was selected because it was determined to be in the mid-range of temperatures at which testing occurred. These values were used as estimations because they are representative of average values and could be applied to a variety of vehicles. In future application of the ANN model this information should be determined for the specific vehicle or vehicle type being examined. Equation 5.5.13 depicts the power correction equation for the values discussed above.

$P_{2}=0.001074 h p+m_{2}\left(\frac{P_{1}-0.001074 h p}{m_{1}}\right)$

Equation 5.5.13

In order to demonstrate the correction for vehicle weight, test runs were conducted employing data obtained from testing of a $525 \mathrm{hp}$ Manufacturer B Engine. The engine was tested at two test weights, $62,360 \mathrm{lbs}$ and 77,980 lbs. During the modeling process the ANN was trained with the data corresponding to the higher weight, and then used to predict emissions corresponding to the lower weight, and then the ANN was trained with the data corresponding to the lighter weight, and used to predict emissions associated with the heavier weight. The following figures depict predicted values for the lighter weight set-up when the ANN was trained with data acquired from testing the vehicle at the heavier weight. Figure 5.5.1 displays the difference between the actual and the predicted engine speed for the lighter weight set-up. 


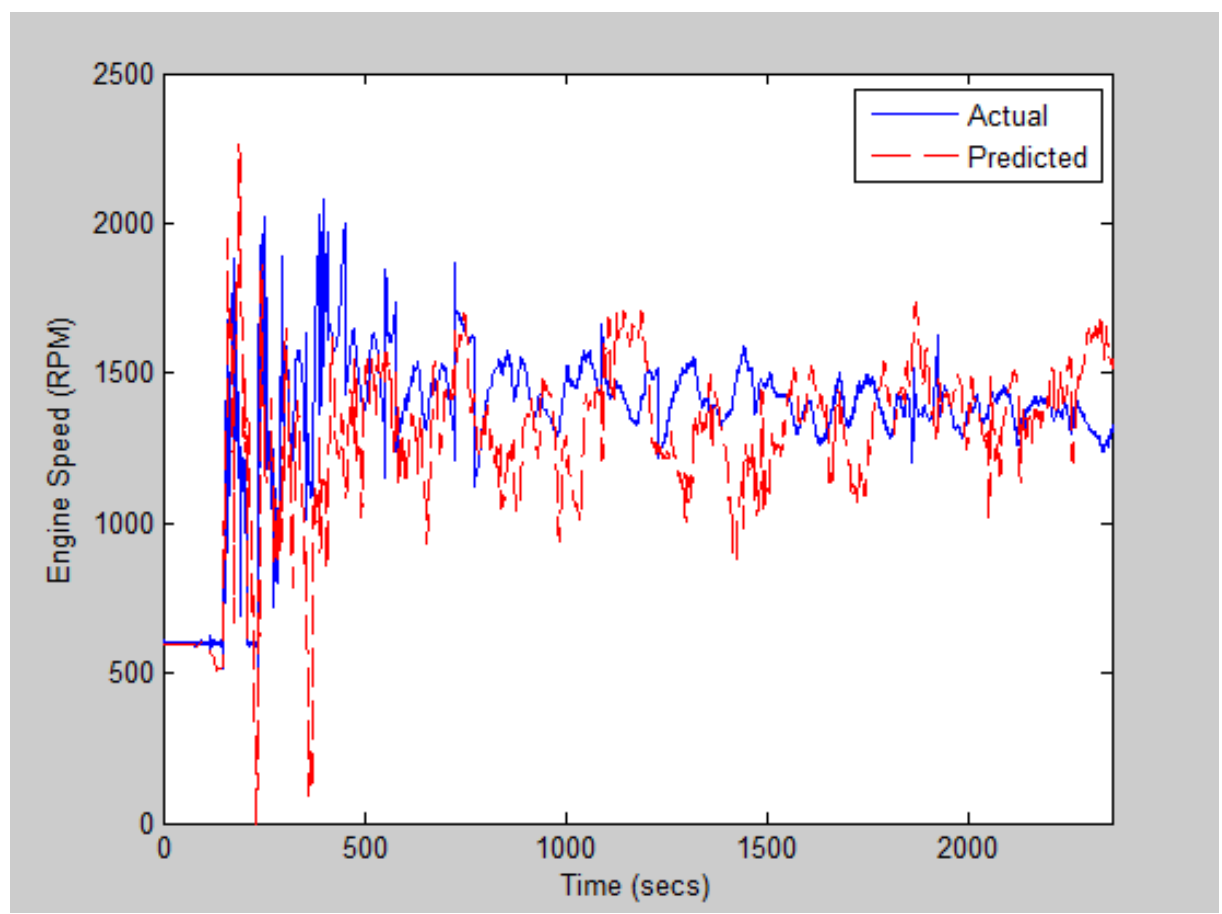

Figure 5.5.1: Comparison of Predicted and Measured Engine Speed for Manufacturer B Engine

Figure 5.5.2 displays the predicted and the measured engine torque for the lighter weight set-up. It is shown that the ANN over and under-predicts the torque for the route being examined. In order to compensate for the weight difference between the training and verification vehicle setups, the scaling factor developed in the previous equations was employed, and the results are shown in Figure 5.5.3. In Figure 5.5.3 it is shown that the scaled engine torque is lower than the predicted engine torque. 


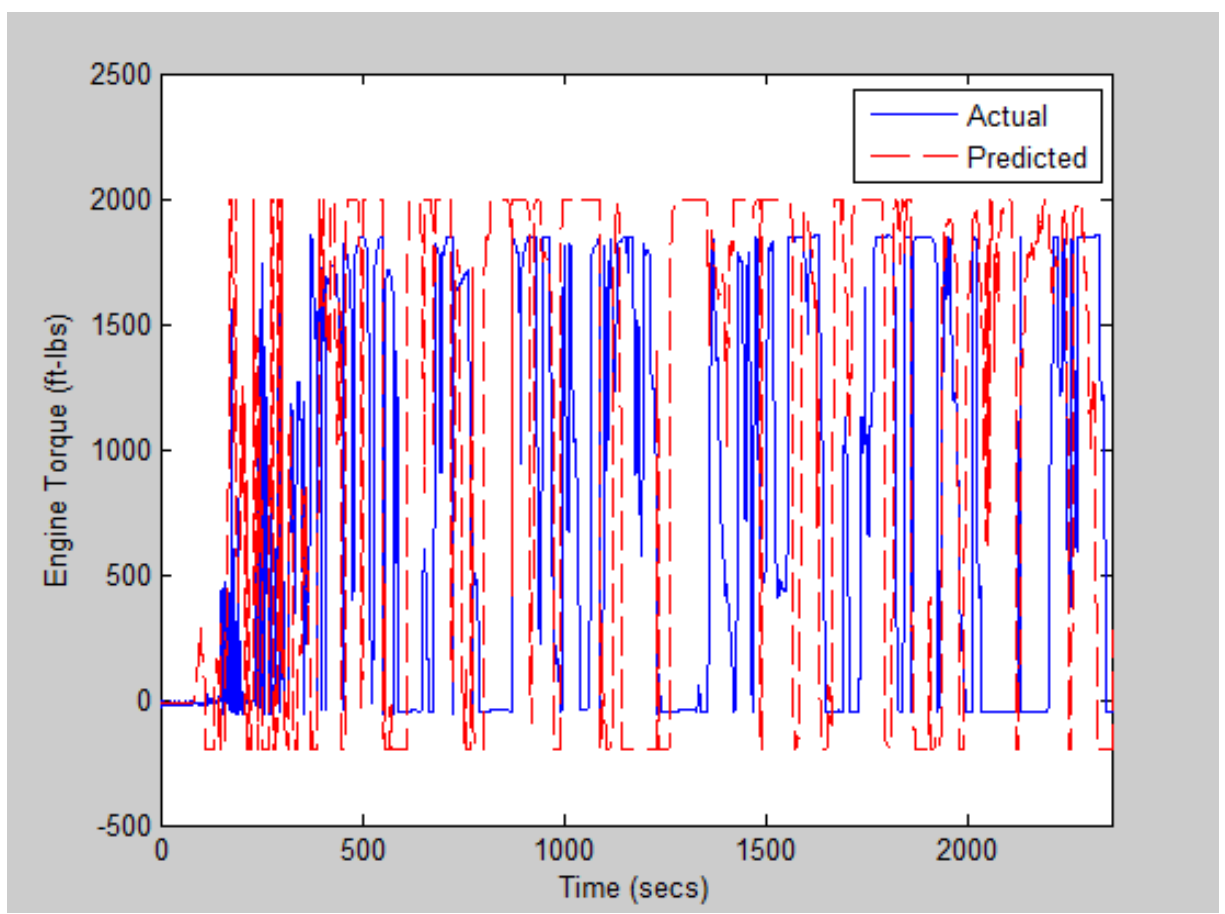

Figure 5.5.2: Comparison of Predicted and Measured Engine Torque for Manufacturer B Engine without Correcting for Weight Difference Between Training and Verification Vehicles

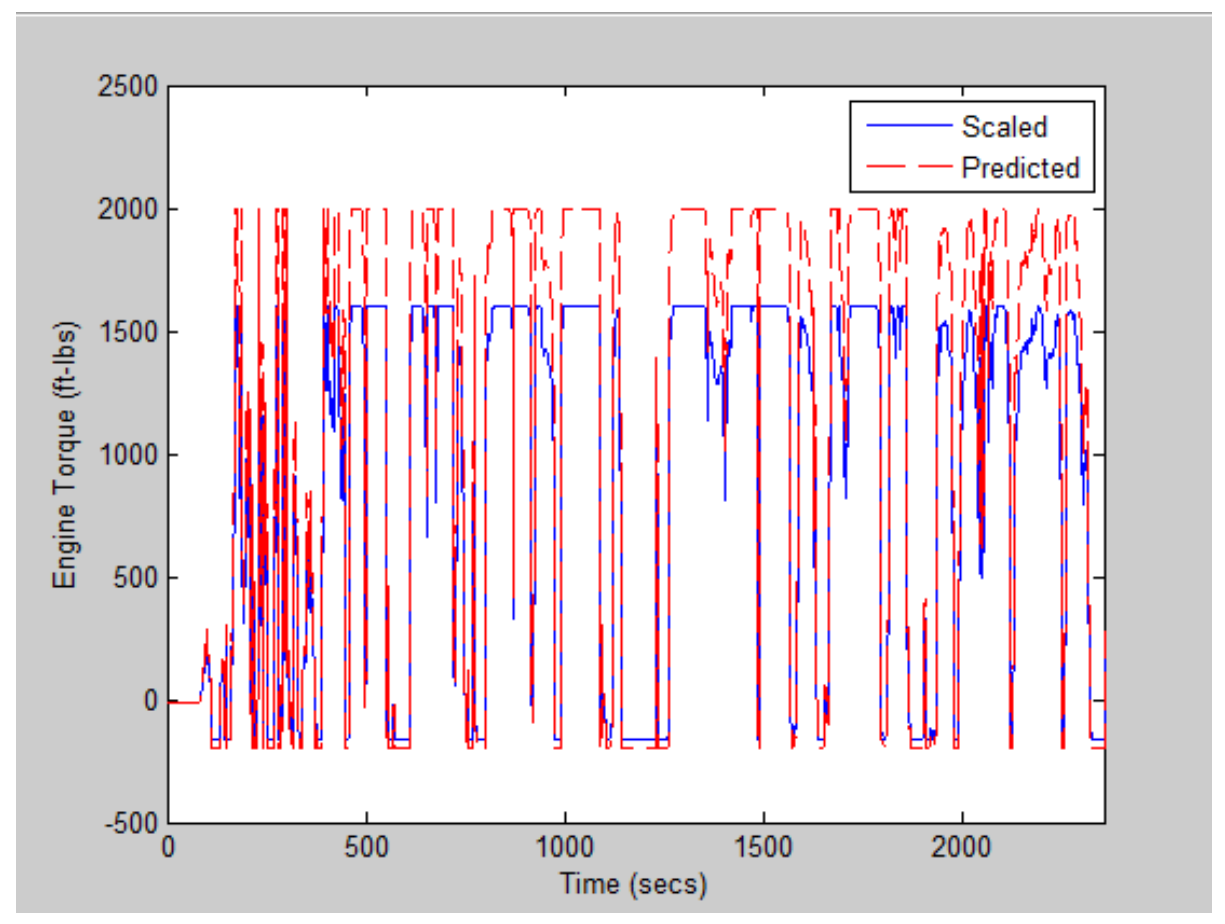

Figure 5.5.3: Comparison of Scaled and Predicted Engine Torque for Manufacturer B Engine when Correcting for Weight Difference Between Training and Verification Vehicles 
A series of training and verification runs were conducted to investigate the accuracy of the scaling factor. Table 5.5.1 displays the difference between the predicted and measured emissions when the ANN was trained with data from the heavier vehicle set-up traveling the route from Bruceton Mills, WV to Sabraton, WV. Data from the lighter weight set-up traveling two different routes was used to verify the ANN's accuracy. Three runs were conducted for each verification weight, where the ANN weights were reinitialized for each run.

It was determined that the average percent differences between measured and predicted emissions for both routes which were examined were lower when the correction for weight was included. When the correction for weight was not included the average percent differences for $\mathrm{NOx}$ and $\mathrm{CO}_{2}$ on the route from Sabraton, WV to Bruceton Mills, WV were 53.1\% and 53.6\%, respectively. When the correction for weight was included, the ANN was able to predict both NOx and $\mathrm{CO}_{2}$ within $18 \%$ of the measured values for the Sabraton, WV to Bruceton Mills, WV route. The differences in accuracy between the predicted and measured emissions when weight was accounted for and not accounted for were not as large for the route from Washington, PA 3 to Sabraton, WV; however when weight was considered the emissions were predicted more accurately.

Table 5.5.1: Comparison of Measured and Predicted Emissions Values for Lighter Weight when Weight Difference is Considered and Not Considered

\begin{tabular}{|c|c|c|c|c|c|c|}
\hline & \multicolumn{3}{|c|}{$\begin{array}{l}\text { Including Corrected Power for } \\
\text { Vehicle Weight }\end{array}$} & \multicolumn{3}{|c|}{$\begin{array}{l}\text { Without Correction for } \\
\text { Vehicle Weight }\end{array}$} \\
\hline \multicolumn{7}{|c|}{ ANN Trained with Bruceton Mills, WV to Sabraton, WV (77,980 lbs) Data } \\
\hline Verification Data & Run & $\begin{array}{l}\text { \% Diff. } \\
\text { NOx }\end{array}$ & $\begin{array}{l}\% \text { Diff. } \\
\mathrm{CO}_{2}\end{array}$ & Run & $\begin{array}{l}\text { \% Diff. } \\
\text { NOx }\end{array}$ & $\begin{array}{c}\% \text { Diff. } \\
\mathrm{CO}_{2}\end{array}$ \\
\hline \multirow{4}{*}{$\begin{array}{c}\text { Sabraton, } W V \text { to Washington, } P A \\
(62,360 \mathrm{lbs})\end{array}$} & 1 & 14.1 & 10.7 & 1 & 38.1 & 24.6 \\
\hline & 2 & 18.3 & 12.0 & 2 & 60.4 & 74.9 \\
\hline & 3 & 20.5 & 13.0 & 3 & 60.8 & 61.4 \\
\hline & Average & 17.6 & 11.9 & Average & 53.1 & 53.6 \\
\hline Verification Data & Run & $\begin{array}{l}\% \text { Diff. } \\
\text { NOx }\end{array}$ & $\begin{array}{l}\% \text { Diff } \\
\mathrm{CO}_{2}\end{array}$ & Run & $\begin{array}{l}\text { \% Diff. } \\
\text { NOx }\end{array}$ & $\begin{array}{c}\text { \% Diff } \\
\mathrm{CO}_{2}\end{array}$ \\
\hline \multirow{4}{*}{$\begin{array}{l}\text { Washington, PA } 3 \text { to Sabraton, } \\
\qquad W V(62,360 \mathrm{lbs})\end{array}$} & 1 & 14.4 & 31.6 & 1 & 45.5 & 53.8 \\
\hline & 2 & 21.2 & 17.9 & 2 & 29.2 & 19.6 \\
\hline & 3 & 31.1 & 21.1 & 3 & 102.5 & 28.4 \\
\hline & Average & 22.2 & 23.5 & Average & 59.1 & 33.9 \\
\hline
\end{tabular}


The data discussed in the previous table was acquired when the ANN was trained with data acquired for a vehicle set-up with a heavier weight than the vehicle employed for verification. Analysis was also conducted when the ANN was trained with data acquired for a lighter weight vehicle set-up and then used to predict emissions associated with a heavier vehicle set-up. It was determined that the weight correction method was not accurate when the ANN was used to predict emissions associated with a heavier weight vehicle than which it was trained. It was recommended that data be obtained for training for loaded vehicles, and then it would be capable of predicting emissions for loaded or unloaded conditions. Percent errors between measured and predicted emissions exceeded $100 \%$ for both $\mathrm{NOx}$ and $\mathrm{CO}_{2}$. It was recommended that the ANN be trained with data that was obtained at vehicle weights equal to or heavier than those vehicle weights for which emissions would be predicted.

\subsection{Deterioration}

As mentioned in the literature review of this document, the emissions produced by an engine are affected by deterioration. The available data was examined in order to determine if a relationship between vehicle odometer reading and produced emissions could be established. In order to establish this relationship, data for a 2002 Manufacturer B engine was examined. This engine was tested in a 2002 tractor at approximately 60,000 lbs and 80,000 lbs. In-use emissions data was collected when the engine had been operational for approximately 244,000 miles, and then again when the engine had been in operation for approximately 462,000 miles. In-use testing occurred at 60,000 lbs and 80,000 lbs for three routes, Washington, PA 1, Washington, PA 2, and Washington, PA 3. In order to examine a trend, ratios of emissions at each tested odometer reading to the emissions at the lowest tested odometer reading were plotted versus the ratio of each tested odometer reading to the lowest tested odometer reading. Figure 5.6.1 shows the relationship between these two ratios for NOx. The NOx values employed in this analysis are integrated values for the route being traveled. This data shows a reduction in NOx with increased odometer mileage. Figure 5.6.2 shows the relationship between $\mathrm{CO}_{2}$ emissions and odometer reading. The data available at the time of the research to establish a deterioration factor was in-use data. The concern with employing in-use data in this application was the number of variables could not be isolated to only odometer reading; therefore it cannot be determine conclusively that the trends shown in Figure 5.6.1 and 5.6.2 are due to deterioration. 
Since the data was obtained from in-use testing, ambient temperature, pressure, and humidity conditions were not controlled, and affected the emissions at the different test times. Also, driver-to-driver variability, traffic conditions, and instrumentation setup could have resulted in difference in measured emissions values, in addition to deterioration. It is recommended that data resulting from engine laboratory testing be employed to determine deterioration effects. This way the variables other than odometer reading can be controlled, and the relationship between odometer reading and emissions produced can be established.

Once a deterioration factor was developed it would be incorporated into the third module of the model. The emissions predicted by ANN2 would be scaled by the developed emissions factor. A deterioration factor was not included in the model due to the inability to isolate the impact of deterioration from ambient affects in the data.

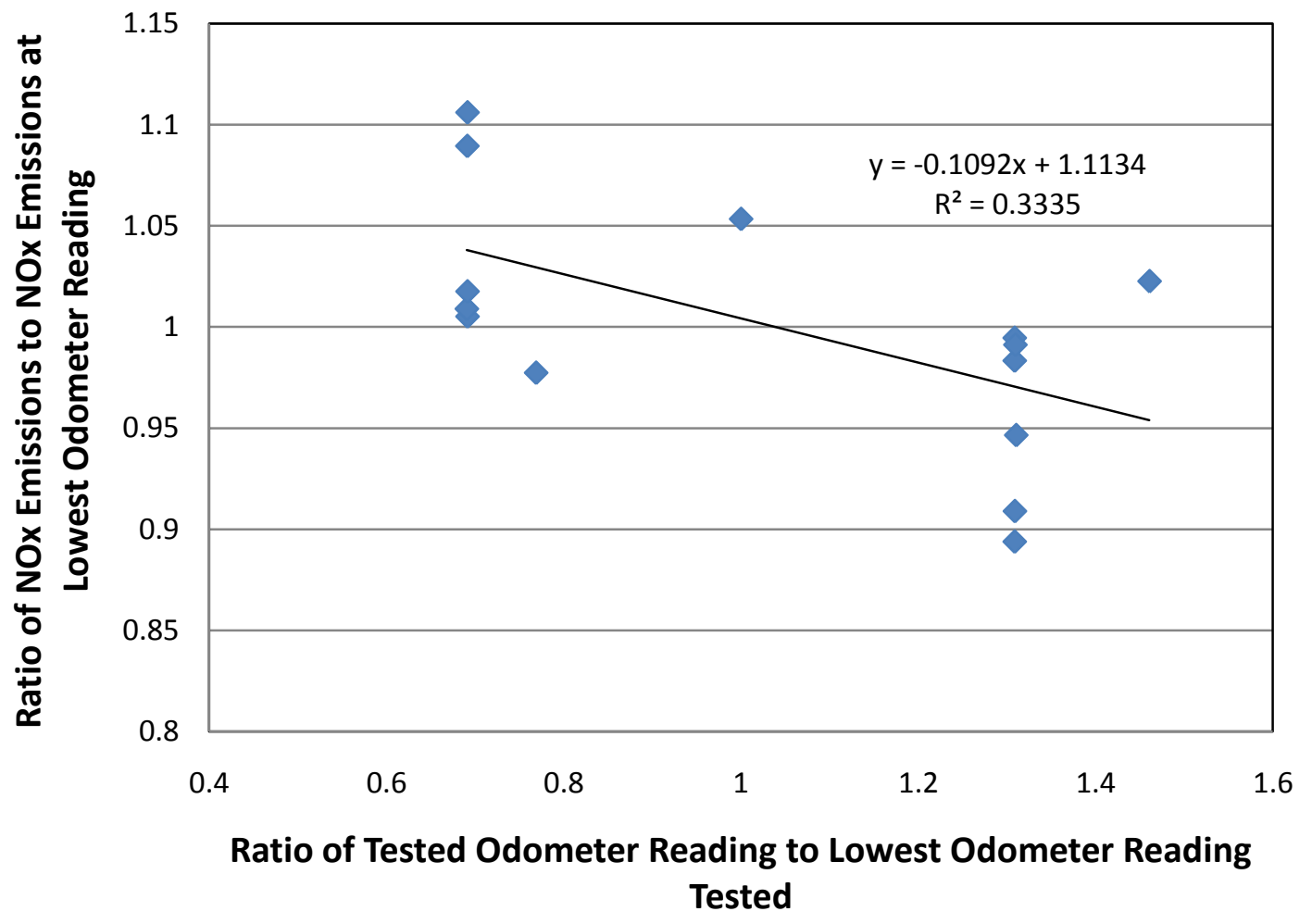

Figure 5.6.1: NOx Emissions versus Odometer Reading 


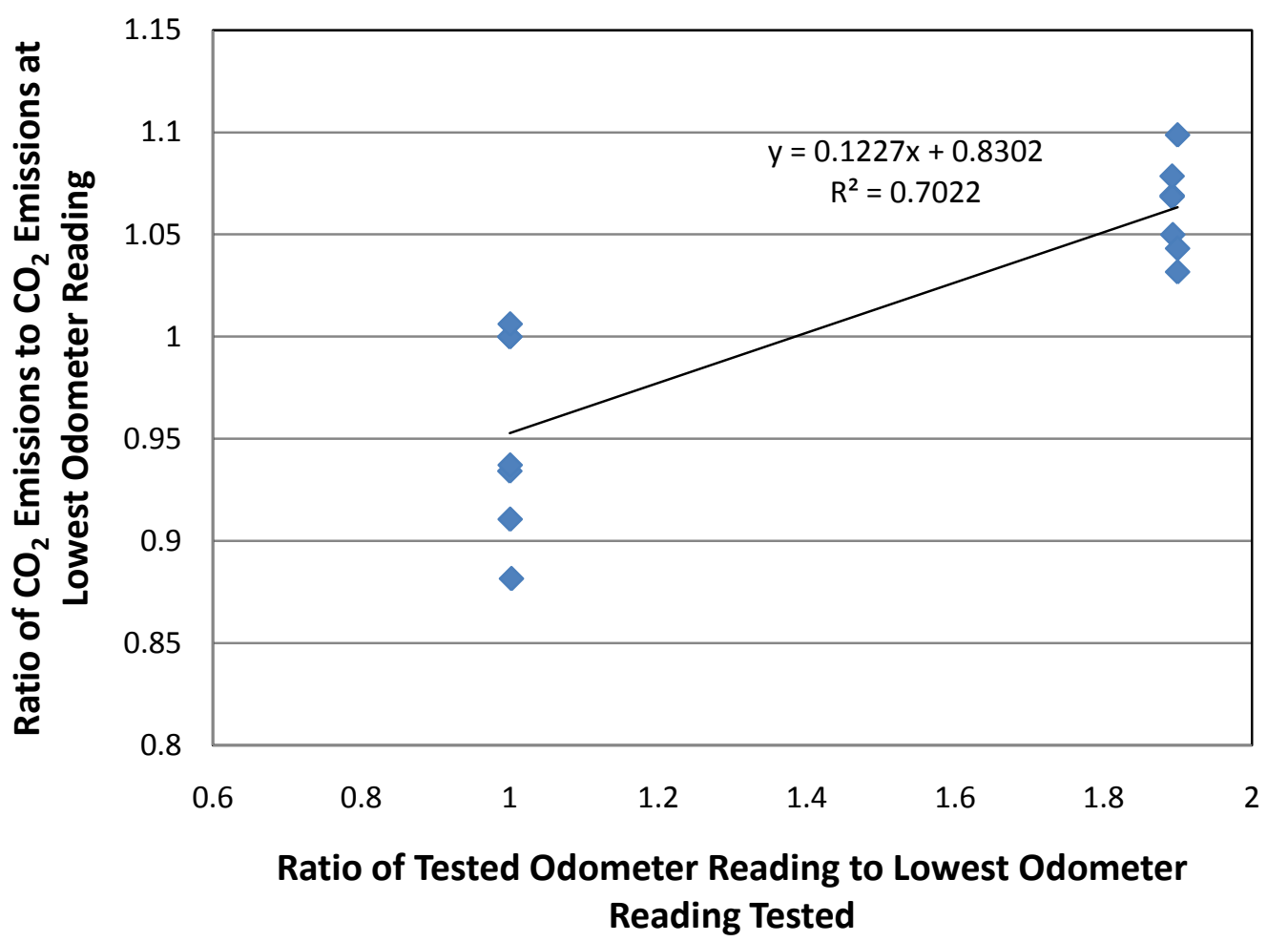

Figure 5.6.2: $\mathrm{CO}_{2}$ Emissions versus Odometer Reading

\subsection{Ambient Condition Effects}

Ambient conditions such as temperature, pressure, and humidity affect the amount of emissions produced by an engine. Temperature and humidity can affect the emissions production at the incylinder level, but also impacts the power consumed by the radiator fan. It should be noted that when examining emissions for inventory modeling purposes the impact of ambient conditions on in-cylinder emissions is negligible compared to radiator fan power consumption. The relationship between ambient temperature and integrated emissions values was examined. In order to establish this relationship, data for a 2002 Manufacturer B engine were examined. This engine was tested in a 2002 tractor at approximately 60,000 lbs and 80,000 lbs. In-use testing for this engine was performed at temperatures ranging from $43^{\circ} \mathrm{F}$ to $68^{\circ} \mathrm{F}$. Ratios of the integrated emissions at each temperature to the average integrated emissions were plotted versus the ratio of the testing temperature to the average temperature. Figure 5.7.1 shows the ratio of NOx produced as a function of temperature ratio. The ratio of $\mathrm{CO}_{2}$ produced as a function of temperature ratio is shown in Figure 5.7.2. It is important to note that deterioration effects are 
also present in this data, since significant mileage occurred between the testing that occurred at low temperature and the testing that occurred at high temperatures. It should also be noted that the power consumption of auxiliary units such as air conditioning systems was also responsible for differences in emissions production for routes run at different ambient conditions.

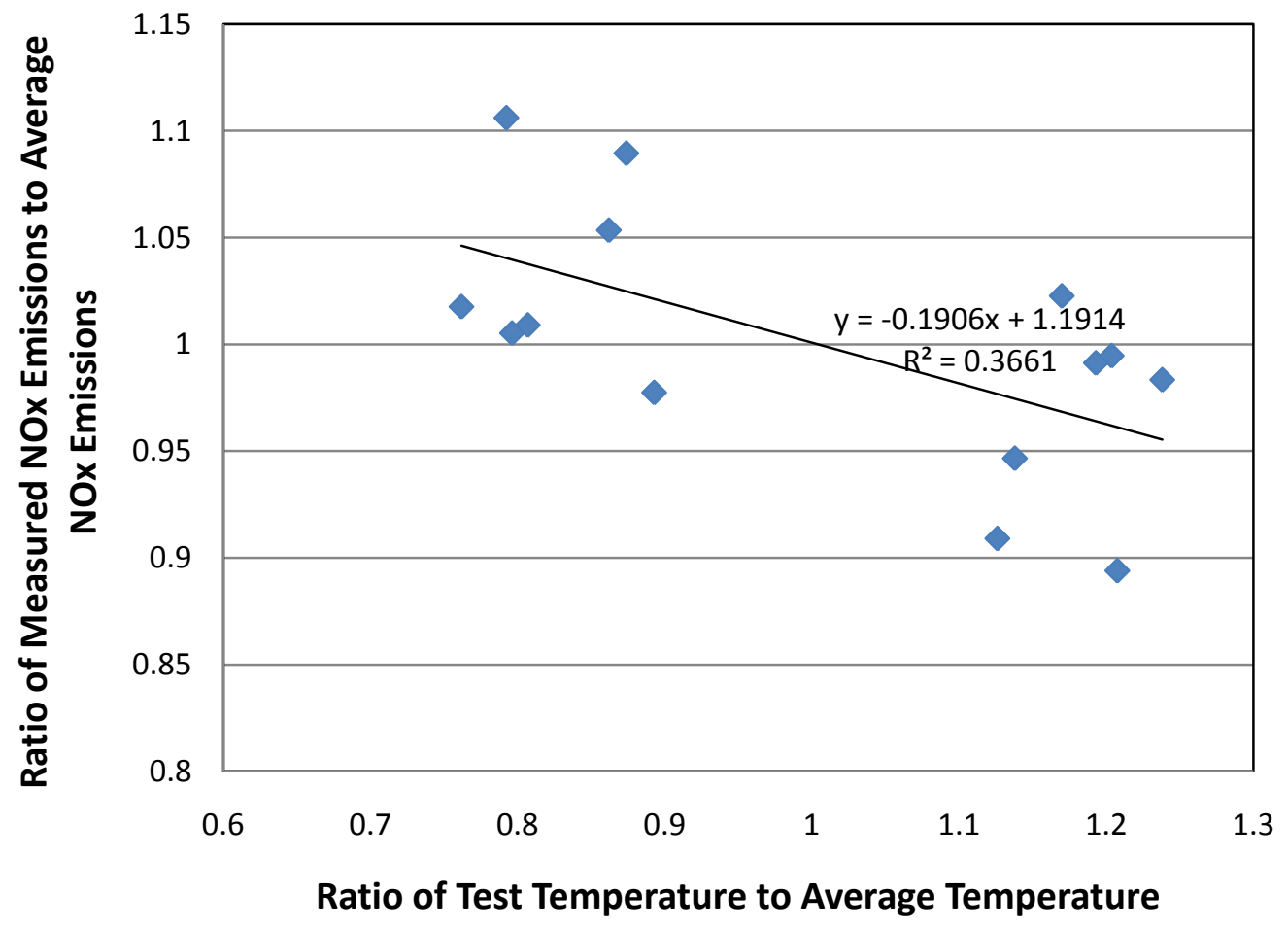

Figure 5.7.1: NOx Emissions versus Temperature 


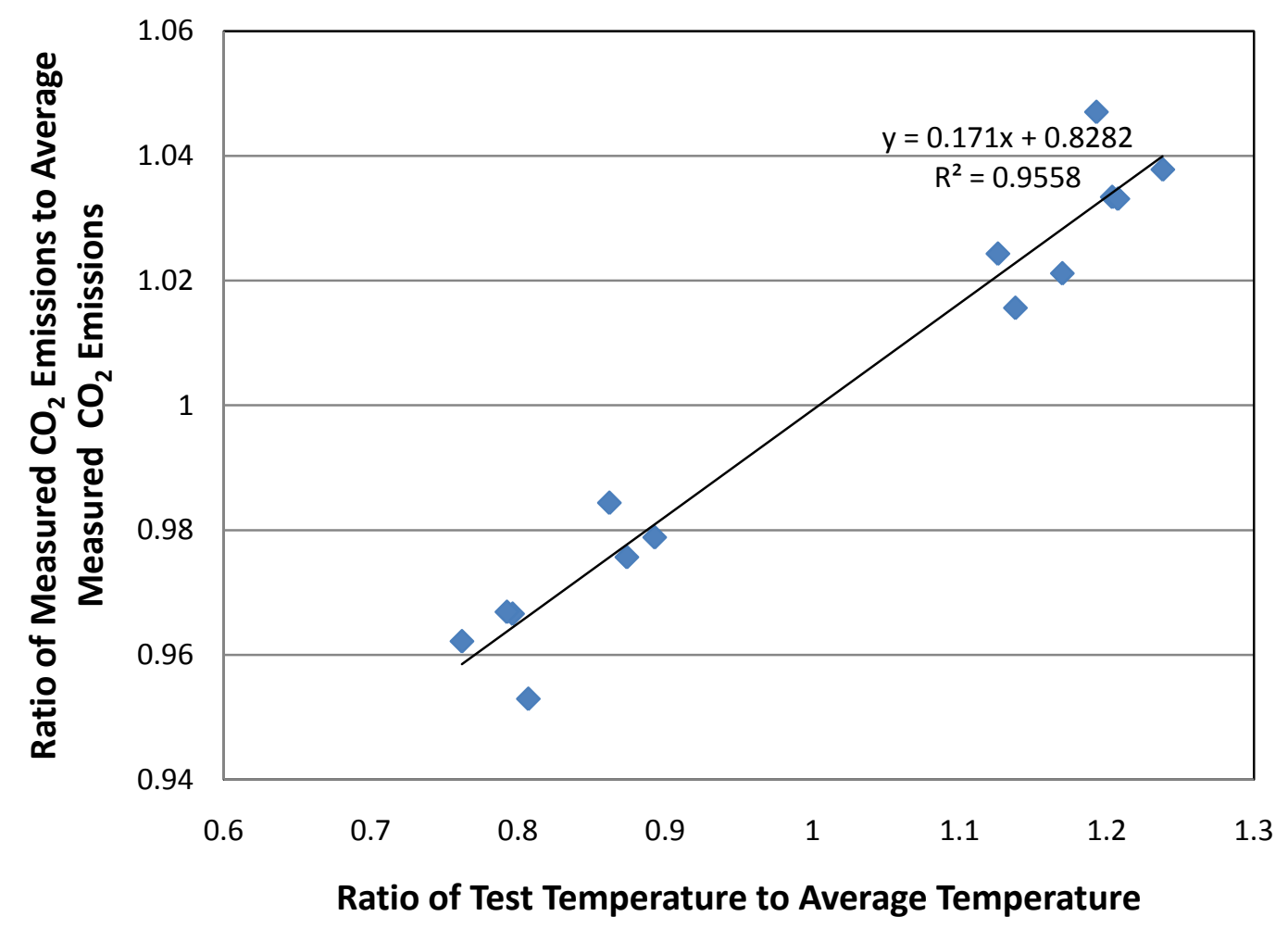

Figure 5.7.2: $\mathrm{CO}_{2}$ Emissions versus Temperature

\subsection{Optimization}

\subsubsection{ANN1}

In order to determine the optimum architecture for the ANNs, a review of literature was conducted, and then a series of test cases were analyzed. The most cited disadvantage of artificial neural networks is the inability to determine if the optimum model has been achieved, and the difficulty in determining which factors affect the accuracy of a model due to the "black box" nature of the operations of an ANN [69]. This section describes a summary of the optimization trials that were conducted.

Table 5.8.1.1 shows the effects of varying input pre-processing techniques, such as smoothing and differentiating on the accuracy of the ANN. The values in this table were determined by altering the inputs to ANN1, while keeping ANN2 unchanged. Various structures for the network were also examined, including differing numbers of layers, and differing numbers of neurons associated with each of those layers. The data shown in the table was acquired when the vehicle module of the ANN was trained with data set Wash PA2 1, and the emissions module of 
the ANN was trained with engine dynamometer data from a cycle designed to simulate the Sabraton, WV to Bruceton Mills, WV route. The data set Wash PA1 1was then employed for model verification. For each change to the network, three test runs were conducted. This method was used, because each time the ANNs were trained, the weights associated with each input and neuron in the layers were re-initialized, which resulted in the trained model reaching a different solution, which would result in different output values. The average percent difference between predicted and measured emissions for the three simulation runs was calculated and used to determine the accuracy of the model. The bottom segment of the table shows that the optimum configuration achieved a percent difference between predicted and measured emissions of less than $8 \%$ for both $\mathrm{NOx}$ and $\mathrm{CO}_{2}$. 
Table 5.8.1.1 Optimization of ANN1

\begin{tabular}{|c|c|c|c|}
\hline \multicolumn{4}{|c|}{ ANN 1 Trained with Wash PA2 1 Data } \\
\hline \multicolumn{4}{|c|}{ ANN 2 Trained with Manufacturer A Sabraton to Bruceton Data } \\
\hline Verification Data & Run & $\%$ Diff. NOx & $\%$ Diff. $\mathrm{CO}_{2}$ \\
\hline \multicolumn{4}{|c|}{ No Smoothing Applied } \\
\hline \multirow{4}{*}{ Wash PA1 1} & 1 & 39.2 & 12.0 \\
\hline & 2 & 41.9 & 13.1 \\
\hline & 3 & 24.6 & 10.6 \\
\hline & Average & 35.2 & 11.9 \\
\hline \multicolumn{4}{|c|}{ No Derivatives Applied, Smoothing Applied } \\
\hline \multirow{4}{*}{ Wash PA1 1} & 1 & 59.5 & 53.6 \\
\hline & 2 & 57.3 & 48.4 \\
\hline & 3 & 93.0 & 60.4 \\
\hline & Average & 69.9 & 54.1 \\
\hline \multicolumn{4}{|c|}{ One Layer of 25 Neurons, Derivatives and Smoothing Applied } \\
\hline \multirow{4}{*}{ Wash PA1 1} & 1 & 28.2 & 30.3 \\
\hline & 2 & 25.3 & 31.1 \\
\hline & 3 & 20.4 & 36.0 \\
\hline & Average & 24.6 & 32.4 \\
\hline \multicolumn{4}{|c|}{ Two Hidden Layers (Derivatives and Smoothing Applied) } \\
\hline \multirow{4}{*}{ Wash PA1 1} & 1 & 38.1 & 12.2 \\
\hline & 2 & 39.7 & 13.4 \\
\hline & 3 & 37.0 & 15.7 \\
\hline & Average & 38.3 & 13.8 \\
\hline \multicolumn{4}{|c|}{$\begin{array}{c}\text { Optimum Configuration (Derivatives, Smoothing, Three Hidden } \\
\text { Layers) }\end{array}$} \\
\hline \multirow{4}{*}{ Wash PA2 1} & 1 & 6.5 & 8.0 \\
\hline & 2 & 1.5 & 8.4 \\
\hline & 3 & 11.1 & 7.2 \\
\hline & Average & 6.4 & 7.9 \\
\hline
\end{tabular}

Initially the inputs were not pre-processed with the moving average smoothing technique. Using the unsmoothed inputs resulted in an average percent difference of $35.2 \%$ for NOx and $11.9 \%$ for $\mathrm{CO}_{2}$ emissions when the predicted values were compared with values measured during in-use testing. It was determined that the moving average smoothing technique could reduce the affect of errors due to dispersion, as well as allow the ANN1 to learn the input to output relationship trends by reducing the number of local extrema. Once it was determined that ANN1 would 
benefit from smoothing, a study was conducted to determine the optimum number of points to average during the smoothing process. Initially the inputs of road grade and vehicle speed were both smoothed once with a set number of points. Through experimentation it was determined that applying moving averages over two different ranges of points resulted in the ANN learning the relationships between the input and the outputs more accurately, therein producing a more accurate prediction of the in-use emissions.

A review of literature pertaining to ANNs suggested that employing derivatives of the input data as inputs as well as the data itself resulted in a more accurate ANN model. Based on the findings of previous ANN research, derivatives of road grade and ECU speed were taken at two different time spans prior to serving as model inputs. When the derivatives were not included as model inputs the average percent difference between the predicted and measured emissions values exceeded $50 \%$ for both $\mathrm{NOx}$ and $\mathrm{CO}_{2}$. It was determined that including the derivatives of the inputs had the greatest affect on the model accuracy. Through trial and error analysis it was determined that for data collected at $10 \mathrm{~Hz}$ the model performed the best when derivatives were taken over 1 and 10 second intervals.

The structure of the ANN1 was also examined. Initially, the ANN was constructed of two layers, the input layer, and one hidden layer consisting of 25 neurons. With this configuration, the average percent difference between measured and predicted $\mathrm{CO}_{2}$ emissions was $32.4 \%$, while the average percent difference was $24.6 \%$ for NOx. In order to improve the performance of the ANN, a second hidden layer of neurons was incorporated. The example data shown in the following table was collected when the first hidden layer had 25 neurons and the second hidden layer housed 10 neurons, it should be noted that various other combination of neuron numbers in the two layers were examined, and resulted in similar accuracy. The best examined configuration for the vehicle ANN was determined to include smoothing the inputs by using two different numbers of points in the moving average, the derivatives of the inputs taken over two different time spans, and three hidden layers of neurons, with 25, 10, and 5 neurons in each layer, respectively. 


\subsubsection{ANN2}

A similar process as discussed above was used to optimize the inputs and structure of the emissions module of the ANN. Table 5.8.2.1 displays the results of some of the optimization techniques applied to ANN2.

Table 5.8.2.1: Optimization of ANN2

\begin{tabular}{|c|c|c|c|}
\hline \multicolumn{4}{|c|}{ ANN 1 Trained with Wash PA2 1 Data } \\
\hline \multicolumn{4}{|c|}{ ANN 2 Trained with Manufacturer A Sabraton to Bruceton Data } \\
\hline Verification Data & Run & $\%$ Diff. NOx & $\%$ Diff $\mathrm{CO}_{2}$ \\
\hline \multicolumn{4}{|c|}{ No Smoothing Applied } \\
\hline \multirow{4}{*}{ Wash PA1 1} & 1 & 37.5 & 35.9 \\
\hline & 2 & 52.4 & 34.1 \\
\hline & 3 & 36.3 & 37.2 \\
\hline & Average & 42.1 & 35.7 \\
\hline \multicolumn{4}{|c|}{ Derivatives Applied, Smoothing Applied } \\
\hline \multirow{4}{*}{ Wash PA1 1} & 1 & 8.9 & 18.3 \\
\hline & 2 & 49.3 & 24.1 \\
\hline & 3 & 22.1 & 56.7 \\
\hline & Average & 26.8 & 33.0 \\
\hline \multicolumn{4}{|c|}{ One Layer of 10 Neurons, Smoothing Applied } \\
\hline \multirow{4}{*}{ Wash PA1 1} & 1 & 35.1 & 38.1 \\
\hline & 2 & 27.2 & 32.0 \\
\hline & 3 & 24.9 & 33.0 \\
\hline & Average & 29.1 & 34.3 \\
\hline \multicolumn{4}{|c|}{ Optimum Configuration (Two layers of Neurons and Smoothing) } \\
\hline \multirow{4}{*}{ Wash PA2 1} & 1 & 6.5 & 8.0 \\
\hline & 2 & 1.5 & 8.4 \\
\hline & 3 & 11.1 & 7.2 \\
\hline & Average & 6.4 & 7.9 \\
\hline
\end{tabular}

Initially the inputs were not pre-processed with the moving average smoothing technique. Using the unsmoothed inputs resulted in an average percent difference of $42.1 \%$ for NOx and $35.7 \%$ for $\mathrm{CO}_{2}$ emissions when the predicted values were compared with values measured during in-use testing.

When the derivatives were included as model inputs the average percent difference between the predicted and measured emissions values exceeded $25 \%$ for both $\mathrm{NOx}$ and $\mathrm{CO}_{2}$. It was 
determined that including the derivatives of the inputs had a negative effect on the model accuracy, since lower percent differences were determined between the predicted and measured emissions when derivatives were not included as inputs. The structure of ANN2 was also examined. Initially, ANN2 was constructed of two layers, the input layer, and one hidden layer consisting of 10 neurons. With this configuration, the average percent difference between measured and predicted $\mathrm{CO}_{2}$ emissions was $34.3 \%$, while the average percent difference was $29.1 \%$ for NOx. In order to improve the performance of ANN2, a second hidden layer of neurons was incorporated. The best performing configuration for ANN2 was determined to include smoothing the inputs by using two different numbers of points in the moving average, and two hidden layers of neurons, with 10 and five neurons in each layer, respectively. It was determined that when the best performing configuration of each ANN was employed NOx and $\mathrm{CO}_{2}$ emissions were predicted within $8 \%$ of the measured values.

\subsubsection{Optimal Network}

Figures 5.8.3.1 and 5.8.3.2 display the inputs to each ANN module. Both road grade and vehicle speed were filtered using a moving average. Two different data sets were created from the smoothing process, each with a differing number of points incorporated into the moving average. The inputs into the ANN1 were smoothed with both a 50 point and 200 point moving average. The derivatives of both smoothed versions of road grade and vehicle speed were taken over two different time intervals and those derivatives were employed as inputs. The inputs were normalized before they entered the ANN. The outputs from the first neural network were engine speed and engine torque. 


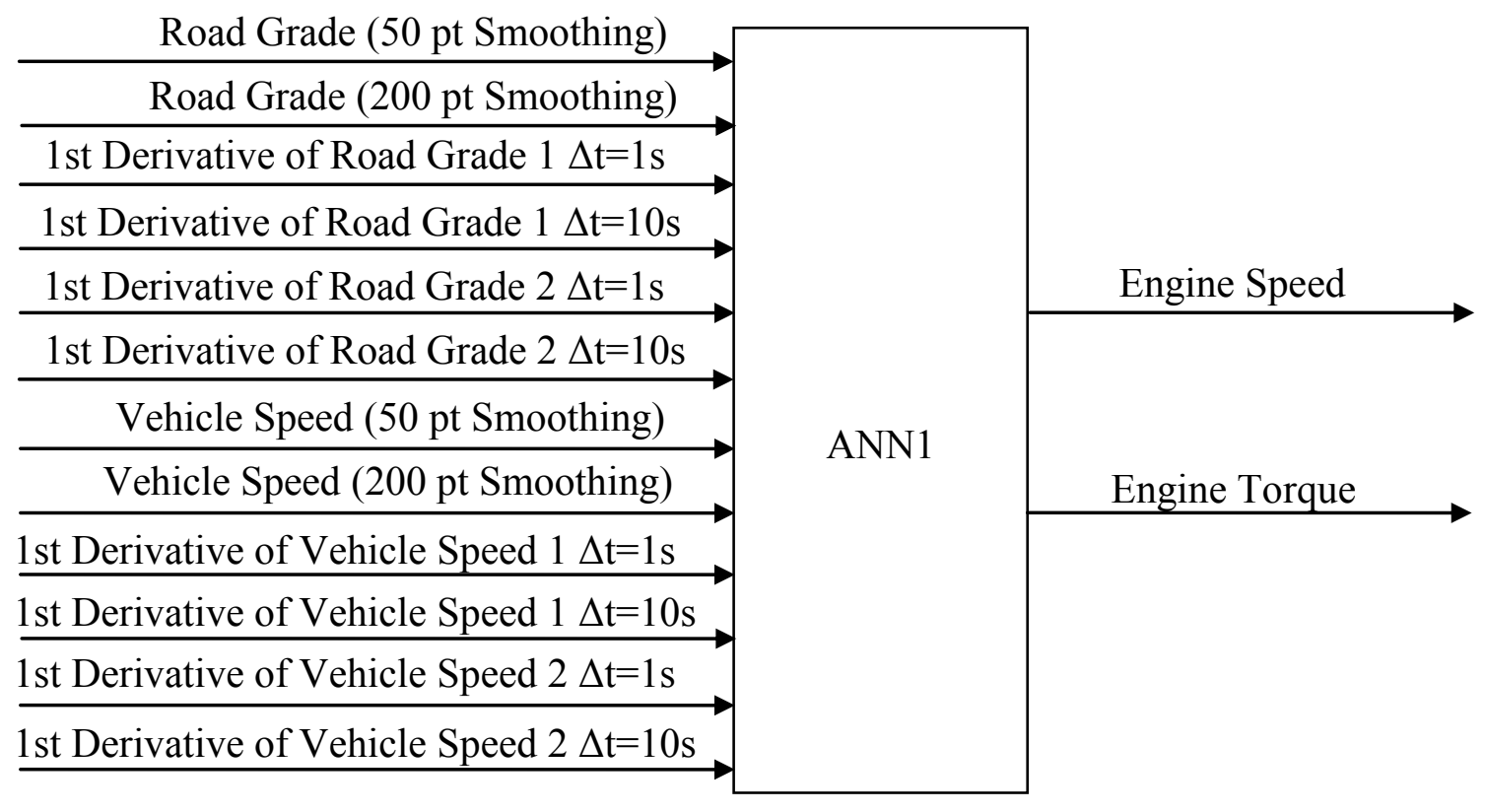

Figure 5.8.3.1: ANN1 Inputs and Outputs

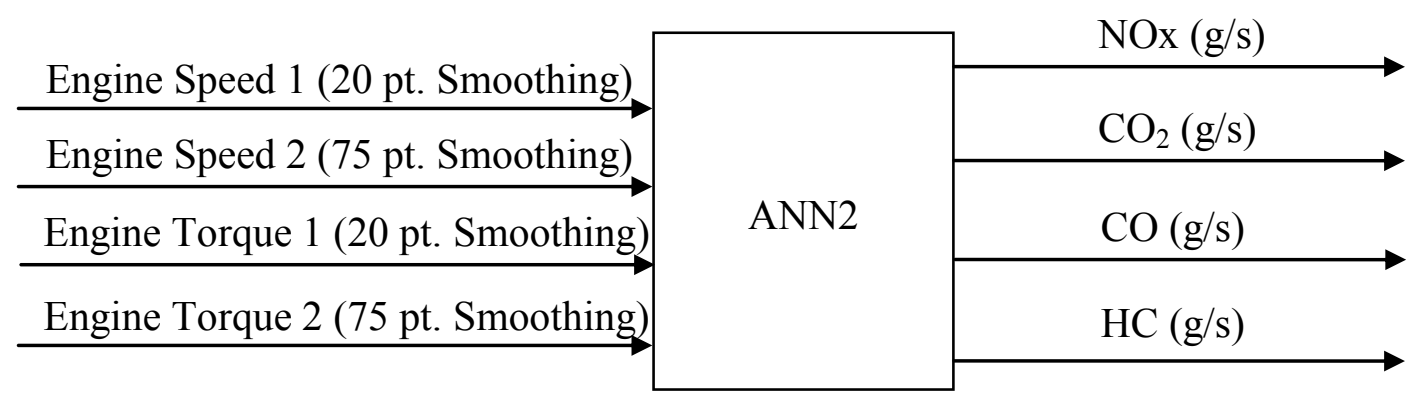

Figure 5.8.3.2: ANN2 Inputs and Outputs

Before serving as inputs to ANN2, the outputs of engine speed and engine torque were normalized, which required the values to be scaled from their actual range, to a range spanning from 0 to 1 . For the scaling calculation, the maximum engine torque for the specific engine was equated to 1, as well as the maximum engine speed. If a predicted engine speed or engine torque exceeded the maximum speed or torque associated with the specific engine being examined, then the engine's maximum value was employed. This is the maximum speed and torque associated with the lug curve of the engine. Prior to training the target emissions outputs were shifted to align with the engine power data. The target outputs were also normalized before they were 
applied to train the ANN. The inputs to ANN2 consisted of the normalized engine speed and engine torque, which were each smoothed by incorporating two different numbers of points into the moving average. The outputs of ANN2 were predicted emission values for $\mathrm{CO}, \mathrm{CO}_{2}, \mathrm{HC}$, and $\mathrm{NO}_{\mathrm{x}}$ on a grams per second basis.

Inputs such as injection timing, in-cylinder pressure, and rail pressure were not employed as inputs due to the availability of data. The network was designed to require as few details about the engine being modeled as possible so that it would be able to be applied to the widest range of applications.

Both the first and second ANNs were structured in a feed-forward back-propagation format. During the development of the ANN various structures were evaluated, however the feedforward back-propagation network proved to be the most applicable due to its ability to predict non-linear relationships. The back-propagation algorithm learns the weights associated with each input and neuron by minimizing the squared error between the output and the training targets. Both ANNs employed the tan-sigmoid transfer function for each hidden layer. A plot of the tan-sigmoid transfer function is shown in Figure 5.8.3.2.

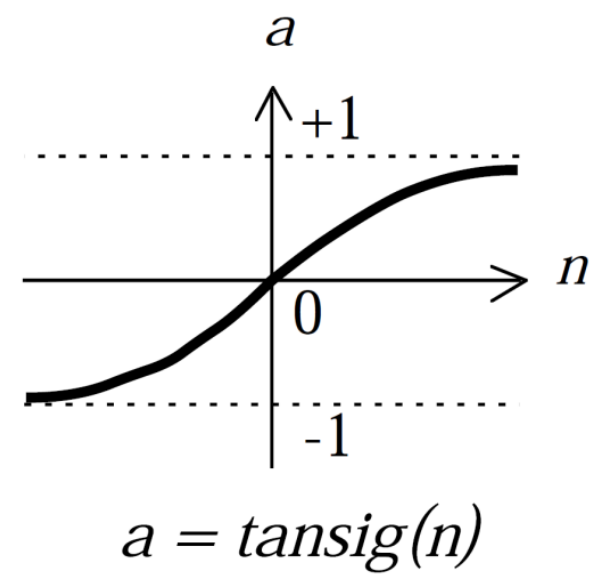

Figure 5.8.3.2: Tan-Sigmoid Transfer Function Plot

The vehicle ANN consisted of three hidden layers of neurons, the first hidden layer housed 25 neurons, the second had 10 neurons, and the final hidden layer consisted of 5 neurons. The first layer of neurons received weights coming from the inputs, and each subsequent layer received 
weights from both the input layer and each previous layer. Through analyzing various numbers of hidden layers and neuron combinations, this format was determined to be the best examined structure for this application. Figure 5.8.3.3 shows a schematic of the neural network architecture employed for the ANN that was responsible for predicting engine speed and torque. The schematic shows that the original inputs as well as the output of the previous layer serve as inputs to the current layer. The blocks designated with " $w$ " in the schematic represent weights, while the blocks designated with a "b" represent any bias that was incorporated into the inputs. For this model no initial biases were employed.

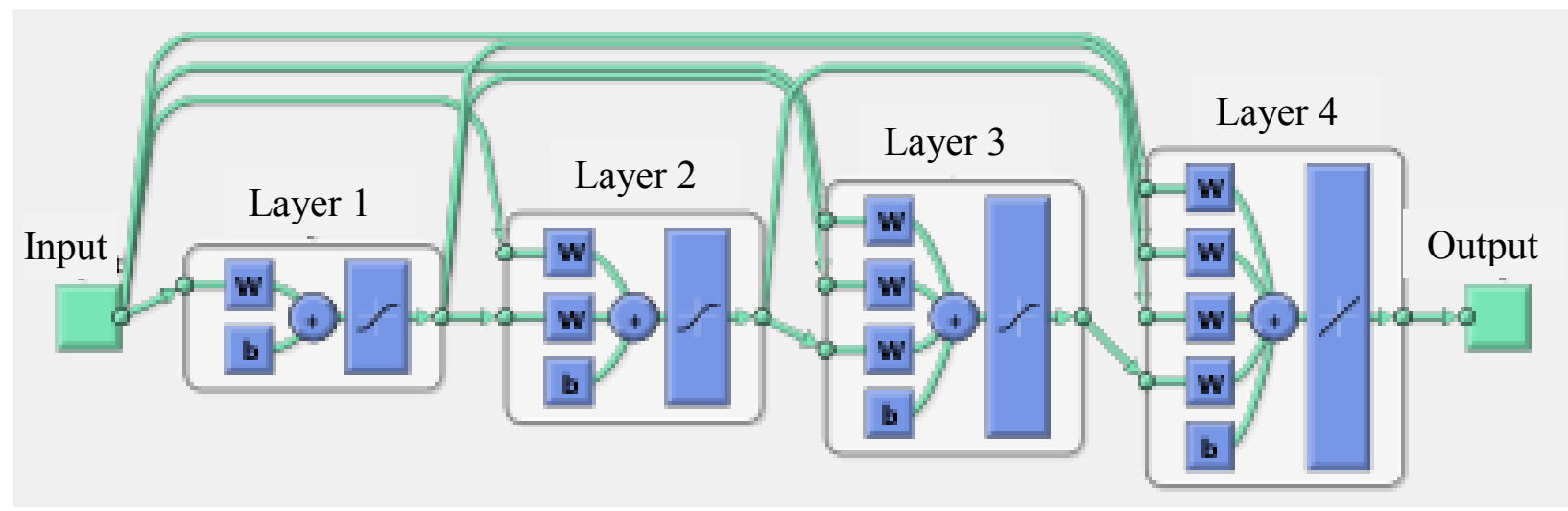

Figure 5.8.3.3: Vehicle ANN, ANN1, Neuron Layer Structure

Figure 5.8.3.4 shows the schematic of the neuron architecture used for the ANN that was designed to predict the emissions associated with the engine speed and torque. This ANN is constructed of three layers, the input layer and two hidden layers made up of 10 and five neurons. It was determined that the emissions ANN did not require more than two hidden layers of neurons due to the reduced number of inputs, and the reduced number of data points in the input vectors since the training data was $1 \mathrm{~Hz}$, and the emissions did not change as rapidly as engine speed and torque. Incorporating more than two layers of hidden neurons in the second ANN resulted in the network over-learning, which decreased its ability to accurately predict emissions on a variety of routes. Over-learning results when the designed network is too complex for the problem. If the network is constructed of too many hidden layers or neurons, it will learn the particular data set which it is being trained with very well, however it will not be able to predict outputs associated with other data sets as well as a less complex ANN would be able to [66]. 


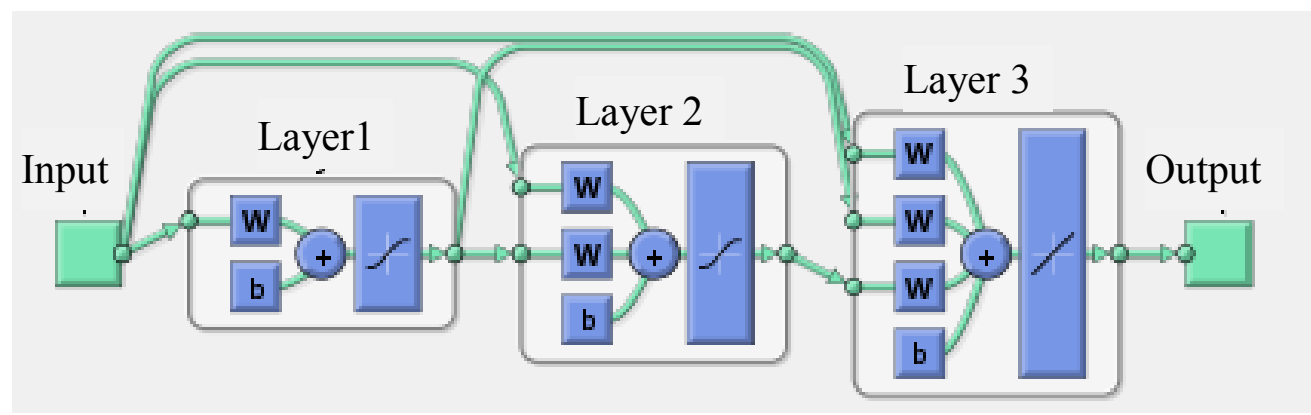

Figure 5.8.3.4: Emissions ANN, ANN2, Neuron Layer Structure

Once each ANN was trained with the training data, a data set that was previously not introduced to the ANN was used as verification data. The verification of the model and the results associated with the finalized version of the ANN are shown in the following chapter of this document. 


\section{Model Verification}

In order to verify the accuracy of the ANN model, a variety of training and verification runs were conducted. This section discusses the percent differences between the integrated emissions between the measured values from in-use testing and the predicted values from the ANN model. It should be noted that the emissions are compared on an integrated basis; therefore the effects of dispersion are minimized in the integrated emitted value, but may be apparent in the continuous plotted emissions values.

\subsection{Repeated Bruceton Mills, WV Route}

In order to show that the ANN model could accurately predict emissions as a function of road grade and vehicle speed, a series of verification tests were conducted. Initially, the vehicle module of the ANN model was trained with data from the Bruceton Mills, WV route, and the emissions module of the ANN was trained with data from the Bruceton Mills, WV dynamometer cycle. The vehicle ANN was trained with in-use data acquired on the route from Bruceton Mills, WV to Sabraton, WV, and the emissions module was trained with engine dynamometer data from the cycle that represented the travel from Sabraton, WV to Bruceton Mills, WV. Separate data sets obtained from different runs of the Bruceton Mills, WV group were then used to verify the accuracy of the ANN. Each time each ANN was trained the weights were initialized with different initial values; therefore the ability of the combined ANN to accurately predict the emissions differed with each training. Once it was established that a model was optimally trained, and predicted the emissions with the highest possible accuracy, the weights and structure of that ANN were saved. The ANN was then applied to other data sets to validate its accuracy.

In order to determine if the ANN could accurately predict the emissions associated with a particular route three data sets from the Bruceton Mills, WV route were employed. Two data sets were from the route that began in Sabraton, WV and ended in Bruceton Mills, WV, while the third verification data set represented the return route from Bruceton Mills, WV to Sabraton, WV. The inputs to the neural network were taken from these data sets, and the emissions predicted by the ANN were compared to the emissions that were measured with the MEMS during the on-road testing. 
Table 6.1.1 displays the results from the three different runs with each verification data set. The network's weights were reinitialized and it was retrained with the training data for each run of the ANN. The table shows the percent difference between the actual measured emissions data and the predicted emissions for $\mathrm{NOx}$ and $\mathrm{CO}_{2}$, these are the percent differences between the integrated emissions values for the entire route. An average percent difference is displayed in the table, as well as the percent difference associated with each run where the weights were reinitialized. It was determined that when both ANN1 and ANN2 were trained with data from the Bruceton Mills, WV route and cycle, the emissions for the Bruceton Mills, WV route could be predicted with percent differences as low as $0.5 \%$ and $7.2 \%$ for $\mathrm{NOx}$ and $\mathrm{CO}_{2}$, respectively. It was determined that when the vehicle ANN was trained with a data set acquired from a specific direction of a route, that it did not predict the emissions associated with the same direction of the route better than the emissions associated with the route traveled in the opposite direction. For example, the emissions predicted for the route from Sabraton, WV to Bruceton Mills, WV were predicted more accurately than the emissions associated with the route from Bruceton Mills, WV to Sabraton, WV when the vehicle module of the ANN was trained with data from the Bruceton Mills, WV to Sabraton, WV route. 
Table 6.1.1: Verification of ANN for Training with, and Predicting Bruceton Mills, WV Routes

\begin{tabular}{|c|c|c|c|}
\hline \multicolumn{4}{|c|}{ ANN 1 Trained with Bruceton2Sab 2 Route Data } \\
\hline ANN 2 Trained with Manufacturer A Sabraton to Bruceton Cycle Data \\
\hline Verification Data & Run & \% Diff. NOx & \% Diff. CO \\
\hline \multirow{4}{*}{ Sab2Bruceton 1 } & 1 & 7.6 & 10.8 \\
\cline { 2 - 4 } & 2 & 3.8 & 13.6 \\
\cline { 2 - 4 } & 3 & 6.0 & 13.3 \\
\cline { 2 - 4 } & Average & 5.8 & 12.6 \\
\hline \multirow{4}{*}{ Sab2Bruceton 2 } & 1 & 8.1 & 7.2 \\
\cline { 2 - 4 } & 2 & 0.5 & 10.7 \\
\cline { 2 - 4 } & 3 & 2.3 & 12.1 \\
\cline { 2 - 4 } & Average & 3.6 & 10.0 \\
\hline \multirow{5}{*}{ Bruceton2Sab 1 } & 1 & 5.9 & 20.5 \\
\cline { 2 - 4 } & 2 & 11.3 & 23.0 \\
\cline { 2 - 4 } & 3 & 6.3 & 20.6 \\
\cline { 2 - 4 } & Average & 7.8 & 21.4 \\
\hline
\end{tabular}

*The highlighted values are shown in the following figures

Figure 6.1.1 compares the predicted and the measured engine torque when the same data set was used for testing and verification, in this case data set Bruceton2Sab 2. A comparison between predicted engine speed and measured engine speed when the data set Bruceton2Sab 2 was used for both training and verification is shown in Figure 6.1.2. It is demonstrated that when the same data set is used for both training and verification that the ANN closely models the engine speed and torque associated with the route being examined. 


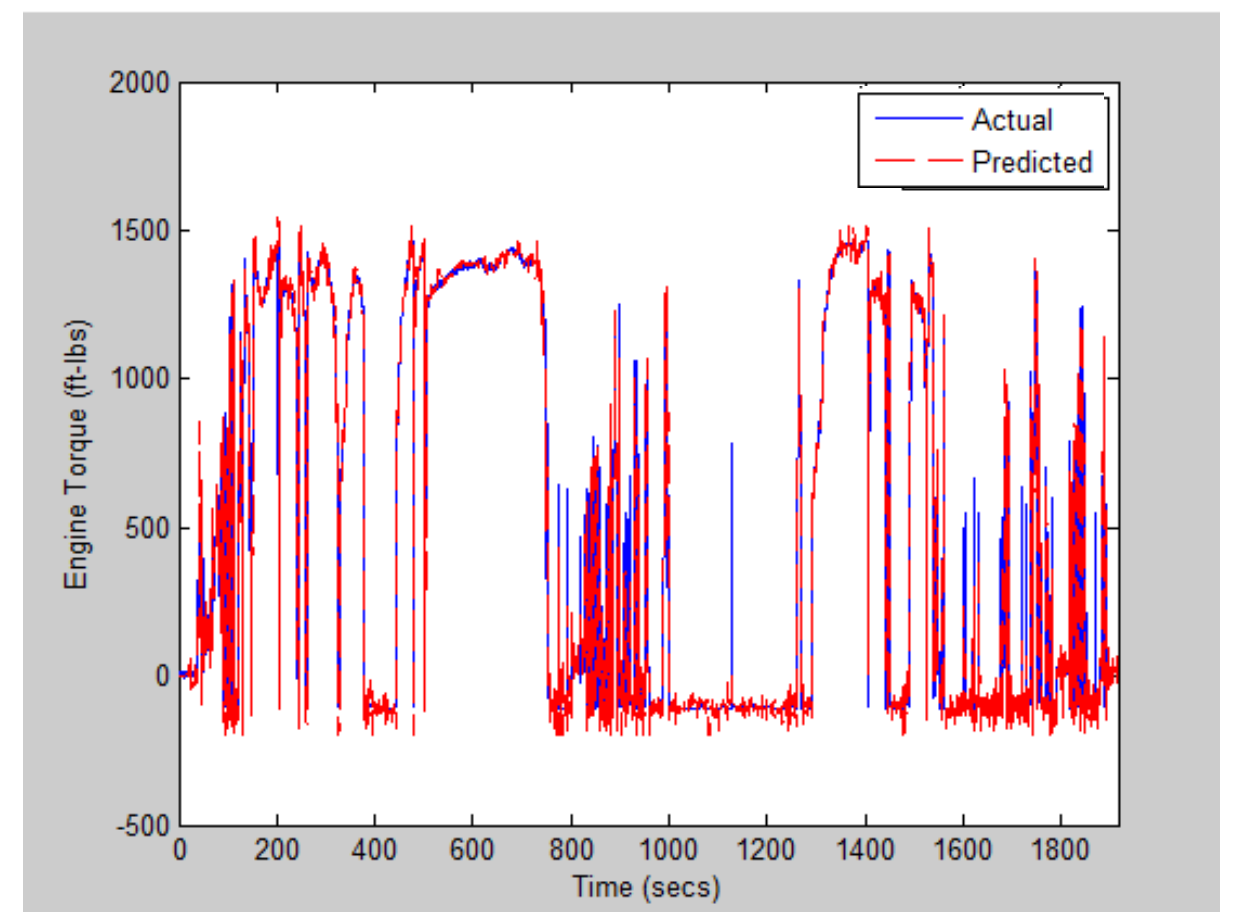

Figure 6.1.1: Vehicle ANN Predicted and Actual Engine Torque When Training with and Predicting Bruceton2Sab 2 Data Set

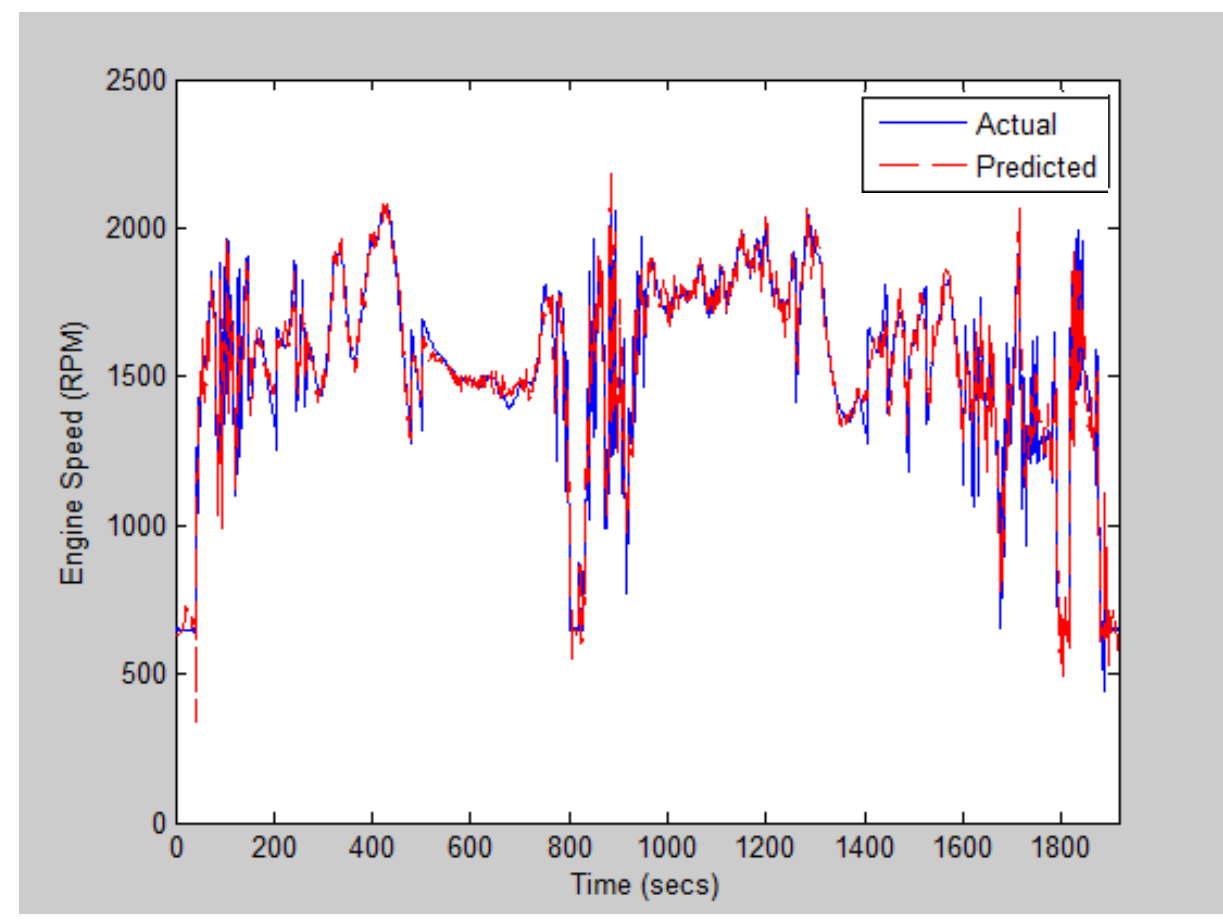

Figure 6.1.2: Vehicle ANN Predicted and Actual Engine Torque When Training with and Predicting Bruceton2Sab 2 Data Set 
Figures 6.1.3 through 6.1.6 compare the values predicted by the ANN to the values that were measured by the MEMS testing for the first verification run with the data set Sab2Bruceton 2, which is highlighted in Table 6.1.1. Figure 6.1.3 shows the difference between predicted engine speed and the measured engine speed, while Figure 6.1.4 shows the difference between the predicted engine torque and the measured engine torque. In the time period between 750 seconds and 1300 seconds, the predicted speed is noticeably lower than the measured speed, and then predicted torque is noticeable higher than the measured torque, this represents the model predicting the wrong transmission gear for this portion of the route. It is also shown that the predicted engine torque exceeds the maximum torque associated with the modeled engine, therefore was limited to the maximum torque value.

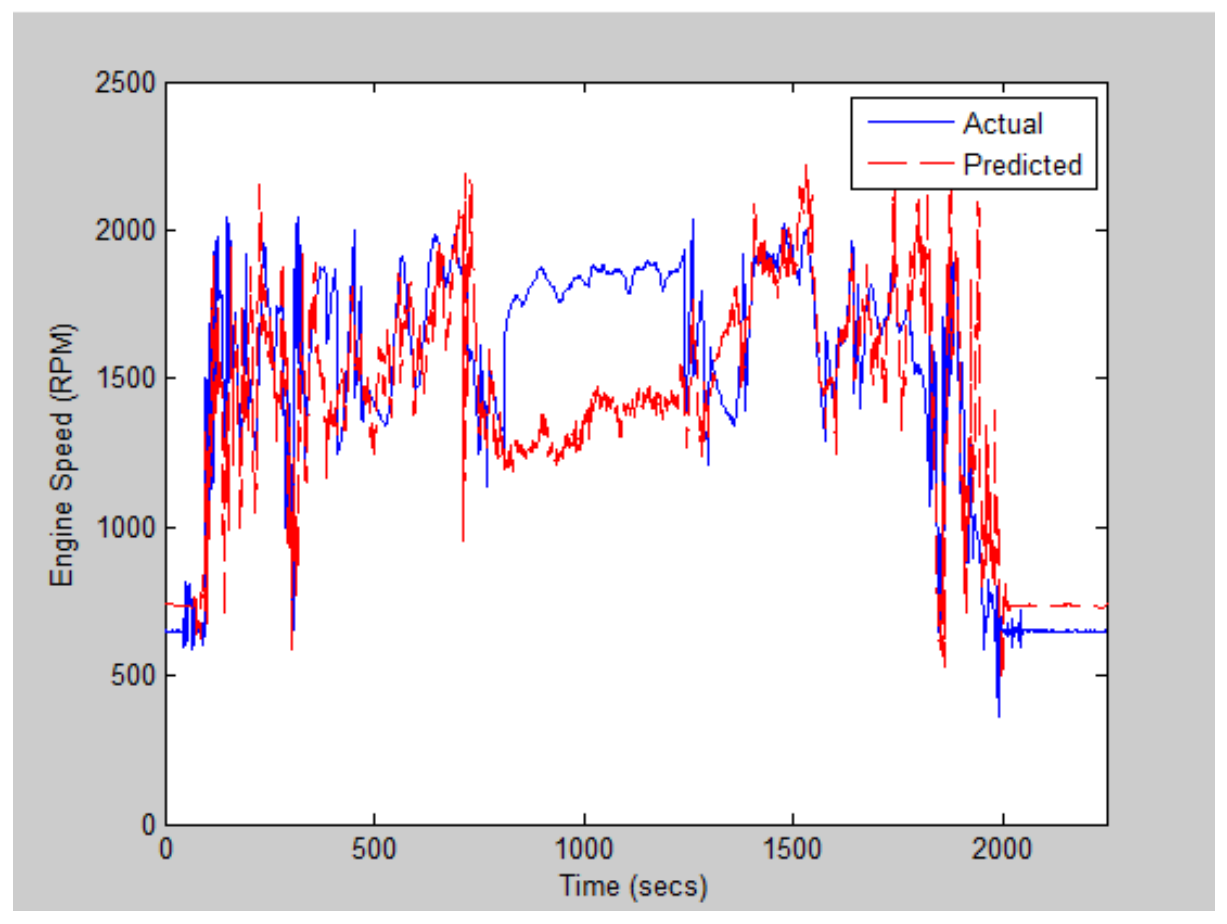

Figure 6.1.3: Difference Between Predicted and Measured Engine Speed for Run 1 Sab2Bruceton 2 Verification Data 


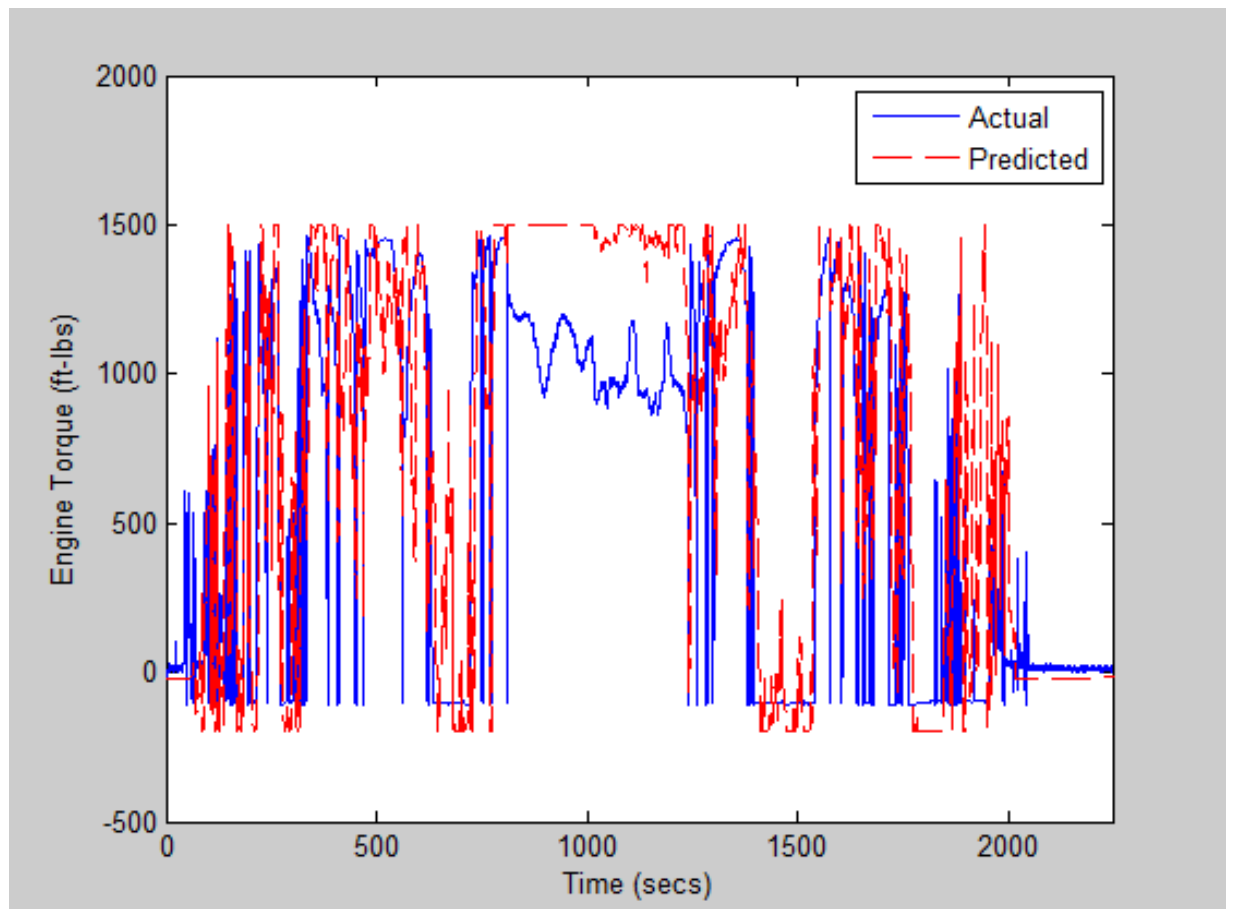

Figure 6.1.4: Difference Between Predicted and Measured Engine Torque for Run 1 Sab2Bruceton 2 Verification Data

Figure 6.1.5 shows the difference between the predicted NOx values and the measured NOx values, while Figure 6.1.6 shows the difference between the predicted $\mathrm{CO}_{2}$ and the measured $\mathrm{CO}_{2}$. It was determined that when the emissions were integrated over the entire cycle that the percent differences for $\mathrm{NOx}$ and $\mathrm{CO}_{2}$ were $8.1 \%$ and $7.2 \%$, respectively. It has been shown that the trends in emission production are similar between the predicted and measured values; however the predicted values over-estimate the quantity of emissions produced, during instantaneous power events. These differences between the predicted and measured were attributed partially to the fact that the two modules of the network were trained with data derived from different types of testing. The emissions module was trained with data acquired in a laboratory with controlled environmental conditions, while the data used to train the vehicle module was obtained in the field, where environmental conditions were not controlled. The mechanisms of measuring the data differed between the in-use and laboratory testing as well, for example the delay and dispersion between a power event and emissions measurement due to the dilution tunnel did not occur in the in-use testing. It was determined that the two modules should 
have the capability of being trained with different data due to the differing availabilities of data for varieties of vehicles. In other words, it increased the flexibility of the model application.

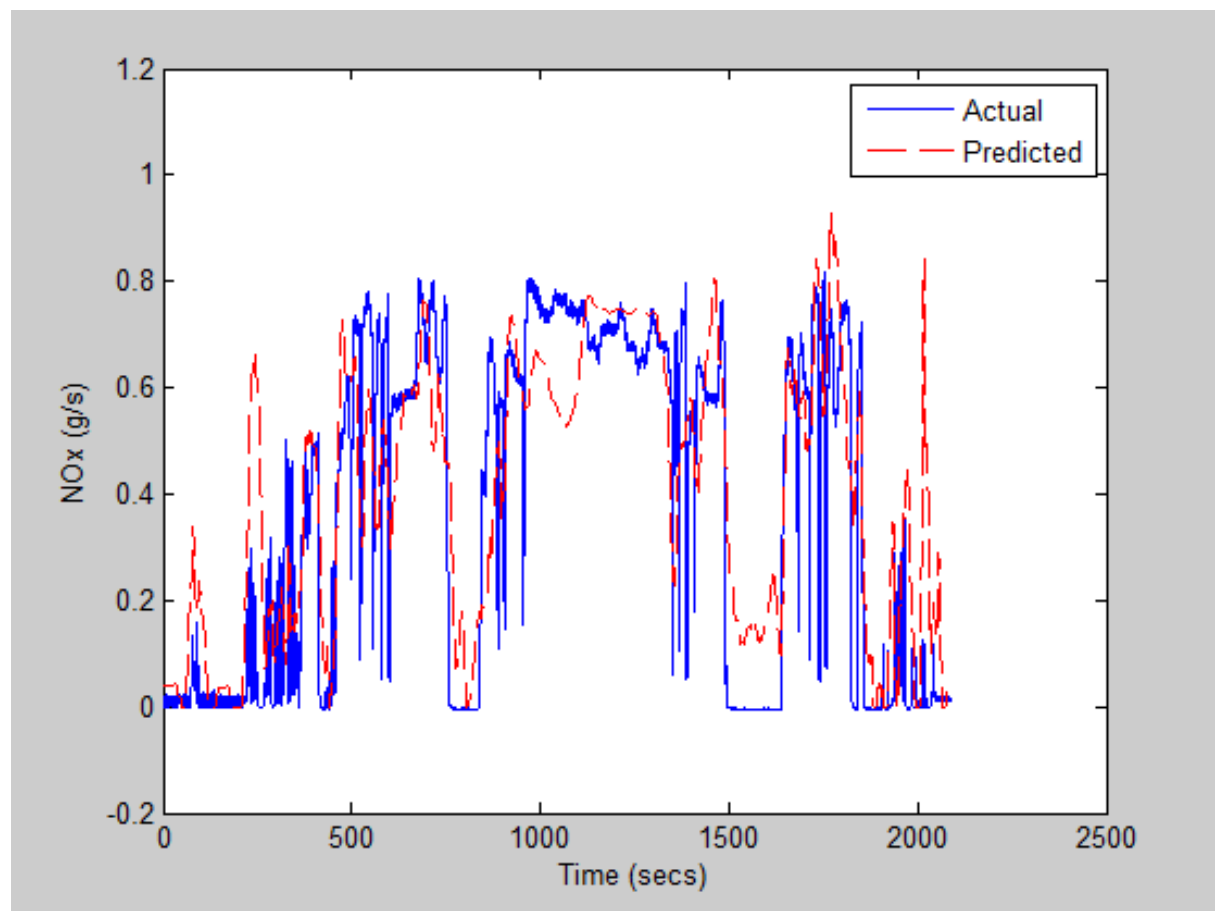

Figure 6.1.5: Difference Between Predicted and Measured NOx Emissions for Run 1 Sab2Bruceton 2 Verification Data 


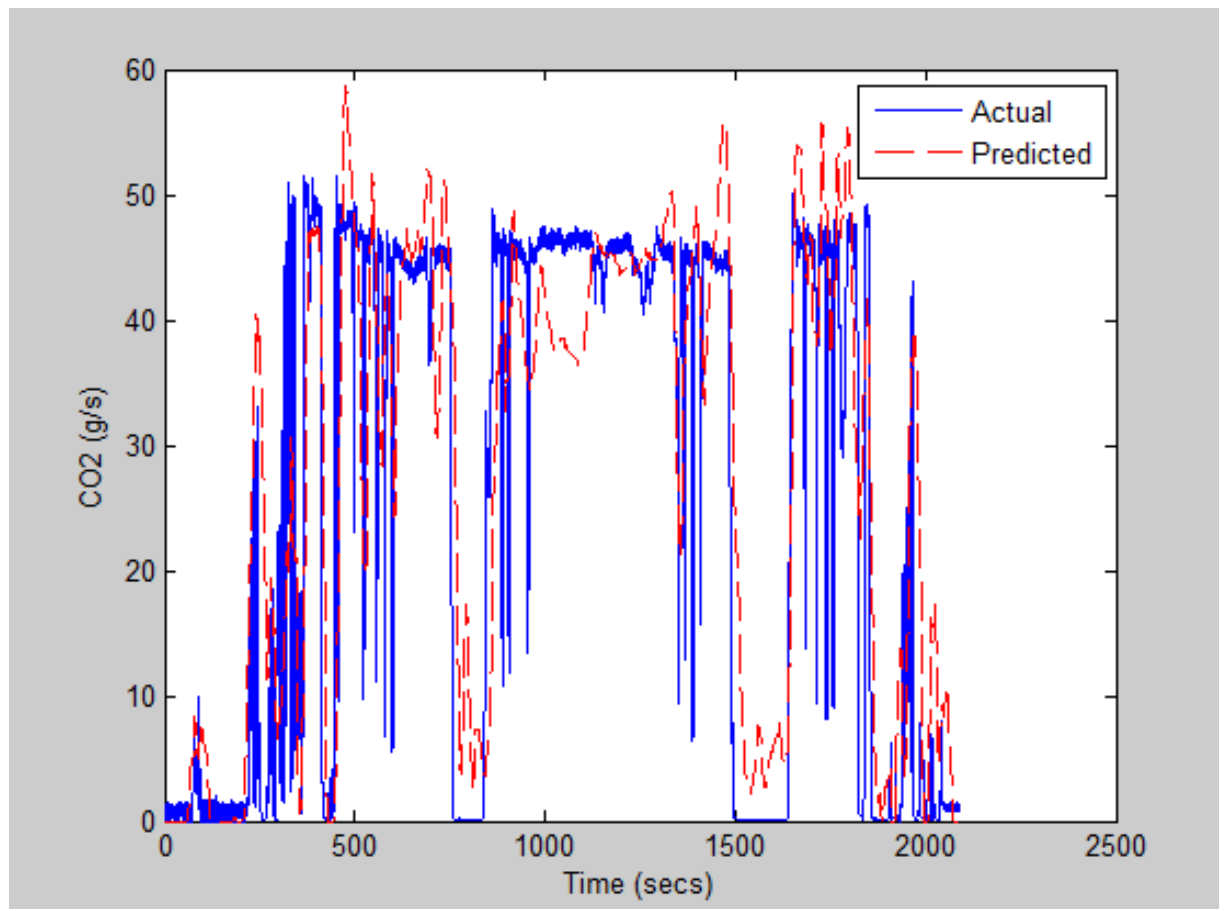

Figure 6.1.6: Difference Between Predicted and Measured $\mathrm{CO}_{2}$ Emissions for Run 1

Sab2Bruceton 2 Verification Data

\subsection{Repeated Washington, PA Route}

In order to demonstrate the ANN's ability to predict emissions for a variety of routes, the Washington, PA route was examined.

Table 6.2.1 shows the results obtained when the vehicle module of the ANN was trained with the Wash PA2 1 data set and the emissions module of the ANN was trained with data obtained from the cycle designed to simulate the Washington, PA route. The trained ANN was then applied to three separate sets of data from Washington, PA routes. One of the verification data sets was acquired during testing of the same route as the training set, Washington, PA 2, and the other two verification data sets represented conditions associated with the Washington, PA 1 route. It was determined that the percent difference between predicted and measured integrated emissions for NOx and $\mathrm{CO}_{2}$ was as low as $1.5 \%$ and $0.6 \%$, respectively. Figures 6.2.1 through 6.2.4 compare the predicted and measured values associated with the second verification run with the data set Wash PA1 1, which has been highlighted in Table 6.2.1. 
Table 6.2.1: Verification of ANN Trained with and Predicting Washington, PA Routes

\begin{tabular}{|c|c|c|c|}
\hline \multicolumn{4}{|c|}{ ANN 1 Trained with Wash PA2 1 Route Data } \\
\hline \multicolumn{3}{|c|}{ ANN 2 Trained with Manufacturer A Washington, PA1 Cycle Data } \\
\hline Verification Data & Run & \% Diff. NOx & \% Diff. CO \\
\hline \multirow{4}{*}{ Wash PA1 2 } & 1 & 17.0 & 38.6 \\
\cline { 2 - 4 } & 2 & 19.4 & 24.7 \\
\cline { 2 - 4 } & 3 & 8.0 & 21.8 \\
\cline { 2 - 4 } & Average & 14.8 & 28.3 \\
\hline \multirow{5}{*}{ Wash PA2 2 } & 1 & 9.6 & 1.5 \\
\cline { 2 - 4 } & 2 & 8.3 & 0.6 \\
\cline { 2 - 4 } & 3 & 13.4 & 3.5 \\
\cline { 2 - 4 } & Average & 10.4 & 1.9 \\
\hline \multirow{5}{*}{ Wash PA1 1 } & 1 & 6.5 & 8.0 \\
\cline { 2 - 4 } & 2 & 1.5 & 8.4 \\
\cline { 2 - 4 } & 3 & 11.1 & 7.2 \\
\cline { 2 - 4 } & Average & 6.4 & 7.9 \\
\hline
\end{tabular}

*The highlighted values are shown in the following figures

Figure 6.2.1 shows the difference between the predicted and measured engine speed for the Washington, PA 1 Route. Overshoot is apparent at and near idle engine speed of $600 \mathrm{rpm}$. The difference between the predicted and measured engine torque for the Washington, PA1 Route is shown in Figure 6.2.2. The ANN was able to predict the trends in engine speed and torque, however the ANN was unable to accurately predict the engine speed and engine torque at idle conditions, as shown in the below figures. Also, in Figure 6.2.2 the ANN was unable to predict the increase in torque that occurred at 900 seconds. The ANN was determined to predict torques below the lowest torques measured in the verification data. The training and verification data was examined to determine if the ANN was attempting to predict data outside the range it was trained on. It was determined that the minimum torque recorded in each of the training and verification data sets was $-111.24 \mathrm{ft}-\mathrm{lbs}$. The ANN was found to predict torques lower than those that it was exposed to in the training data. 


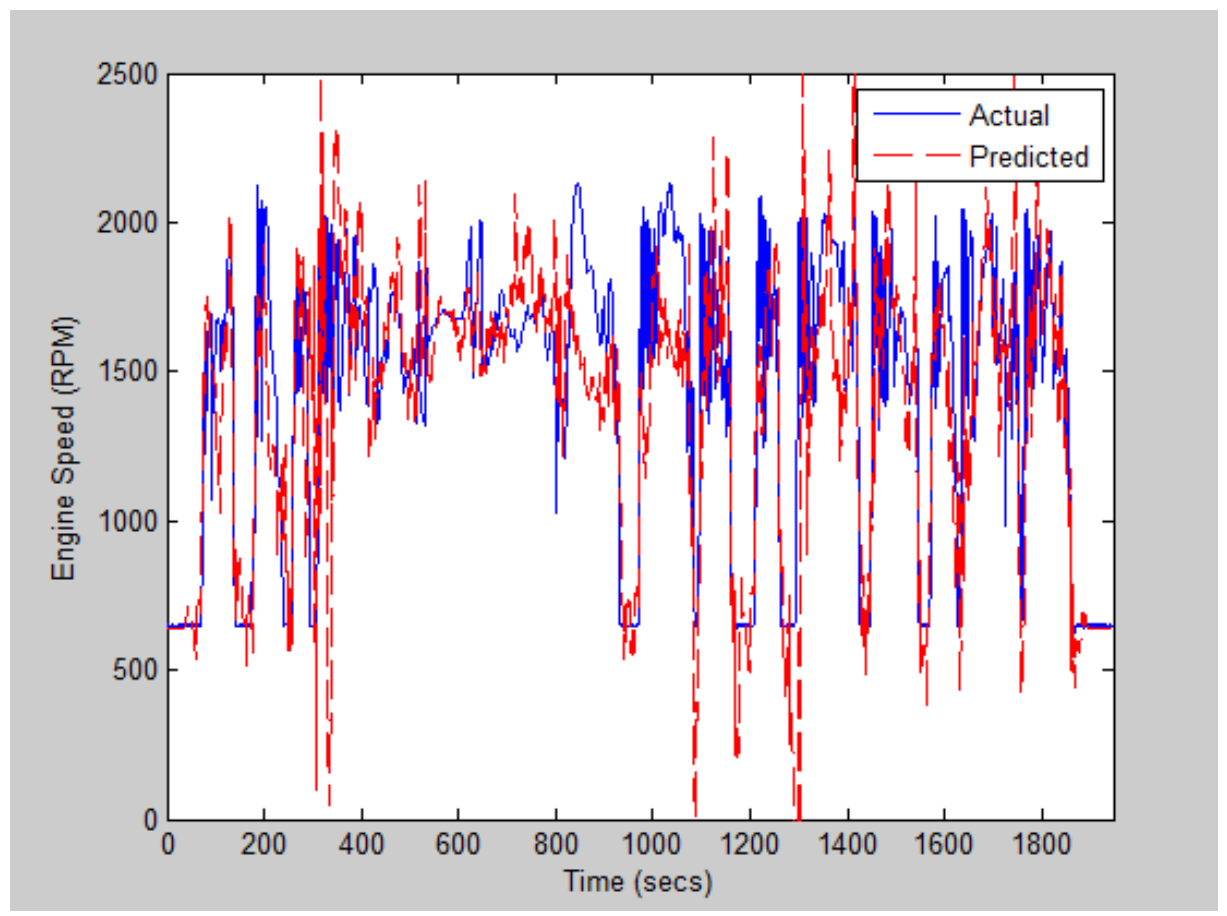

Figure 6.2.1: Difference Between Predicted and Measured Engine Speed for Washington, PA1 When ANN was Trained with Washington, PA2 1

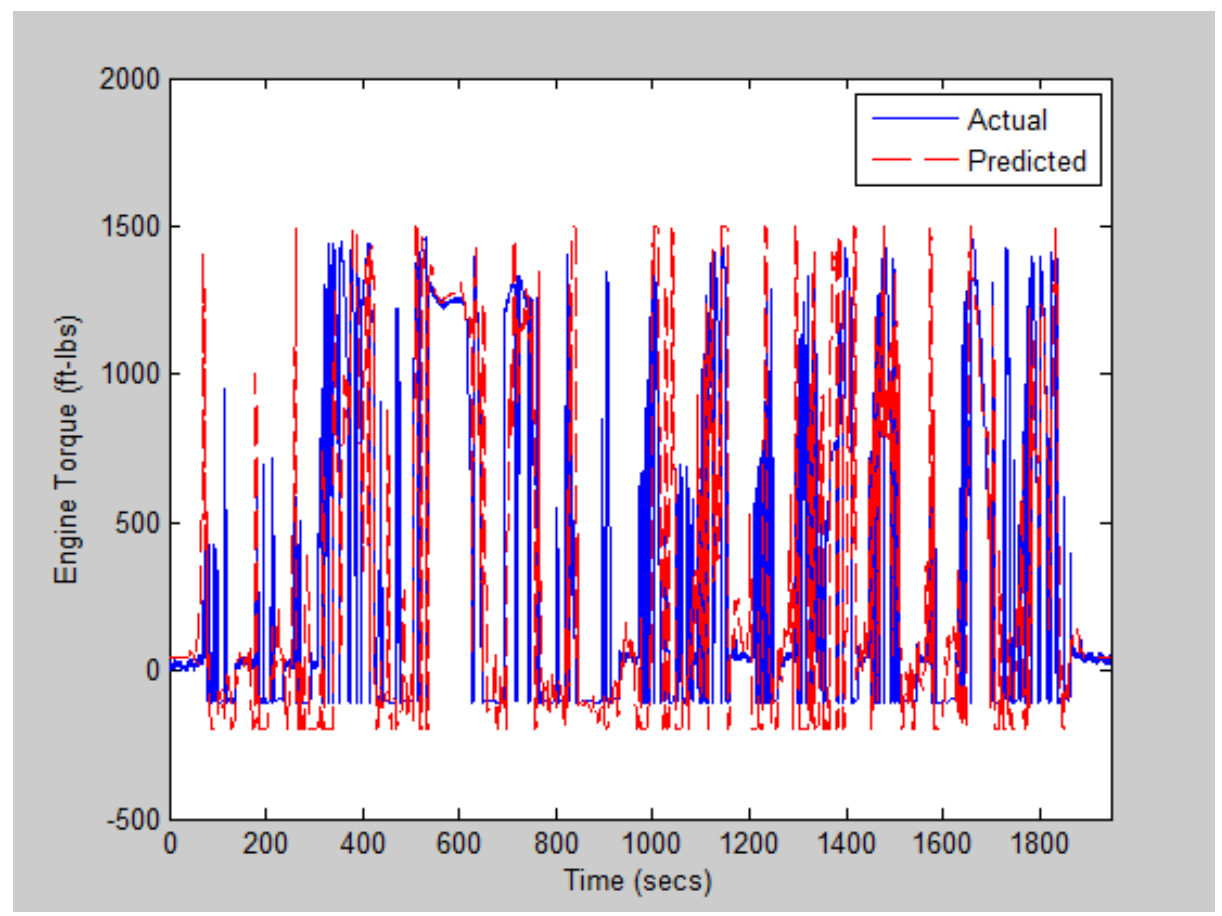

Figure 6.2.2: Difference Between Predicted and Measured Engine Torque for the Washington, PA1 Route When ANN was Trained with Washington, PA2 1 
The difference between the predicted and measured emissions for the Washington, PA1 route are shown in Figures 6.2.3 and 6.2.4. It is shown that the trends in the measured emissions are closely followed by the predicted emissions. The ANN over-predicted $\mathrm{CO}_{2}$ more frequently that NOx, however when the ANN was initialized with different weights, the network was found to over-predict NOx more frequently than $\mathrm{CO}_{2}$, therefore variation in predicted value is attributed to network initialization factors. The variation in the weight initialization is a minor impact on the accuracy of the emissions prediction when compared to the impact of data training quality. It should be noted that once the ANN is trained with a data set, the weights may be saved, and the network can be employed to predict the emissions with an infinite number of data sets. Once the weights are saved and that specific ANN is used to predict emissions the differences in weight initialization have no impact on the emissions predicted by the ANN.

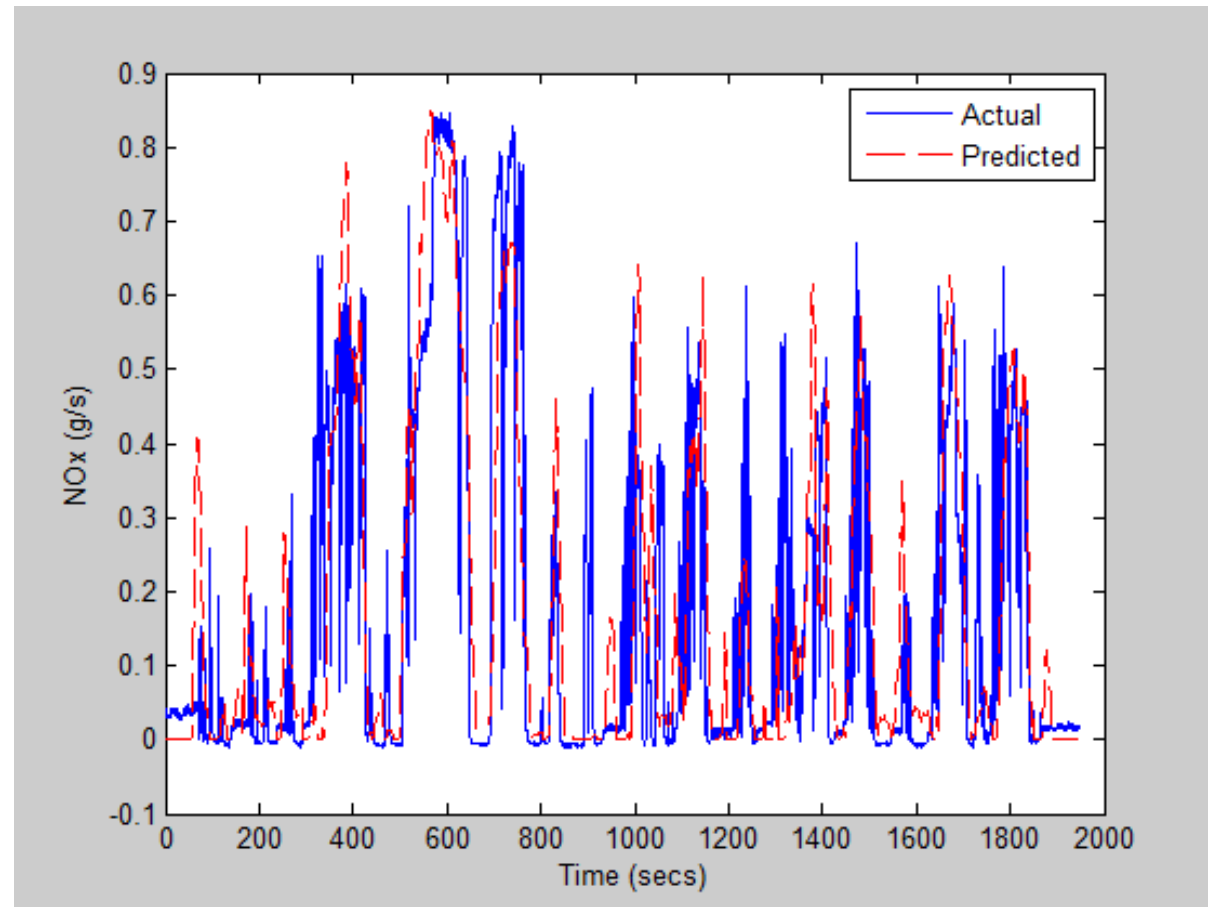

Figure 6.2.3: Difference Between Predicted and Measured NOx Emissions for the Washington, PA1 Route When ANN Was Trained with Washington, PA2 1 


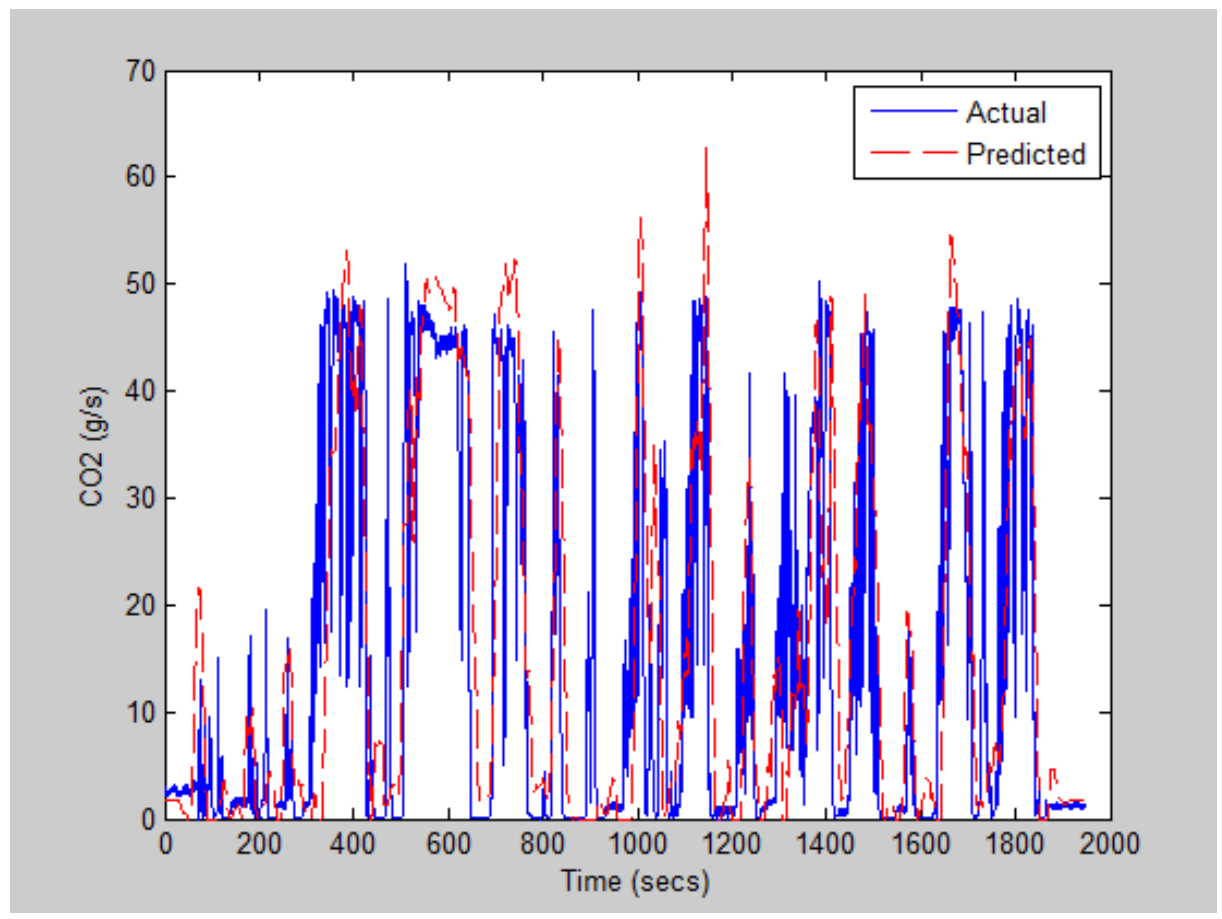

Figure 6.2.4: Difference Between Predicted and Measured $\mathrm{CO}_{2}$ Emissions for the Washington, PA1 Route When ANN Was Trained with Washington, PA2 1

\subsection{Same Engine Different Route}

In order to determine if the ANN was capable of predicting emissions for a route other than that it was trained with, the ANN was trained with data from the Washington, PA route, and then it was employed to predict the emissions associated with the Bruceton Mills, WV route. Table 6.3.1 displays the percent differences for the integrated emissions values. The vehicle ANN was trained with data from the Washington, PA2 route, and the emissions ANN was trained with data from the cycle designed to model the Sabraton, WV to Bruceton Mills, WV route. The ANN was then employed to predict the emissions associated with various runs of the Bruceton Mills, WV route. Two of the verification data sets represent the route traveled from Bruceton Mills, WV to Sabraton, WV, while the third verification data set represents the return route, from Sabraton, WV to Bruceton Mills, WV. It was determined that the ANN training combination mentioned above was able to most accurately predict the emissions associated with the route traveled from Sabraton, WV to Bruceton Mills, WV with an average percent difference between measured and predicted values of $12.3 \%$ and $14.1 \%$ for $\mathrm{NOx}$ and $\mathrm{CO}_{2}$, respectively. In this case the training route was representative of urban driving conditions, while the verification routes 
were representative of rural driving conditions. This difference in route characteristics is thought to contribute to the decrease in accuracy of the ANN to predict emissions.

Table 6.3.1: Verification of ANN Trained with Washington, PA Route, and Used for Prediction of Bruceton Mills, WV Routes

\begin{tabular}{|c|c|c|c|}
\hline \multicolumn{4}{|c|}{ ANN 1 Trained with Wash PA2 1 Route Data } \\
\hline ANN 2 Trained with Manufacturer A Sabraton to Bruceton Cycle Data \\
\hline Verification Data & Run & \% Diff. NOx & \% Diff. CO 2 \\
\hline \multirow{4}{*}{ Bruceton2Sab 2 } & 1 & 21.7 & 29.1 \\
\cline { 2 - 4 } & 2 & 22.7 & 28.2 \\
\cline { 2 - 4 } & 3 & 19.7 & 27.9 \\
\cline { 2 - 4 } & Average & 21.4 & 28.4 \\
\hline \multirow{5}{*}{ Sab2Bruceton 1 } & 1 & 18.3 & 16.4 \\
\cline { 2 - 4 } & 2 & 18.2 & 10.9 \\
\cline { 2 - 4 } & 3 & 0.3 & 14.9 \\
\cline { 2 - 4 } & Average & 12.3 & 14.1 \\
\hline \multirow{5}{*}{ Bruceton2Sab 1 } & 1 & 25.3 & 20.5 \\
\cline { 2 - 4 } & 2 & 25.8 & 23.0 \\
\cline { 2 - 4 } & 3 & 9.0 & 21.5 \\
\cline { 2 - 4 } & Average & 20.0 & 2.9 \\
\hline
\end{tabular}

*The highlighted values are shown in the following figures

Figure 6.3.1 shows the difference between predicted engine speed and the measured engine speed, while Figure 6.3.2 shows the difference between the predicted engine torque and the measured engine torque for run 3 with Sab2Bruceton 1 verification data. It is demonstrated between 800 and 1300 seconds the ANN model predicted a different gear than experienced in the measured route data. The different gear is represented by the lower prediction of speed and higher prediction of torque. This could be due to the fact that driver behavior, and/or traffic conditions differed between data sets that were used for training and verification data.

The ANN over-predicted the engine speed between 1400 and 1600 seconds. It was thought that the ANN was extrapolating data outside the boundaries of which it was trained. It was determined that the maximum engine speed for the training route was $2106.6 \mathrm{rpm}$, and the maximum engine speed during the Sab2Bruceton 1 training route was $2046.7 \mathrm{rpm}$. It was shown 
that at engine speeds close to the upper bounds of training data, the ANN would over-estimate the engine speed.

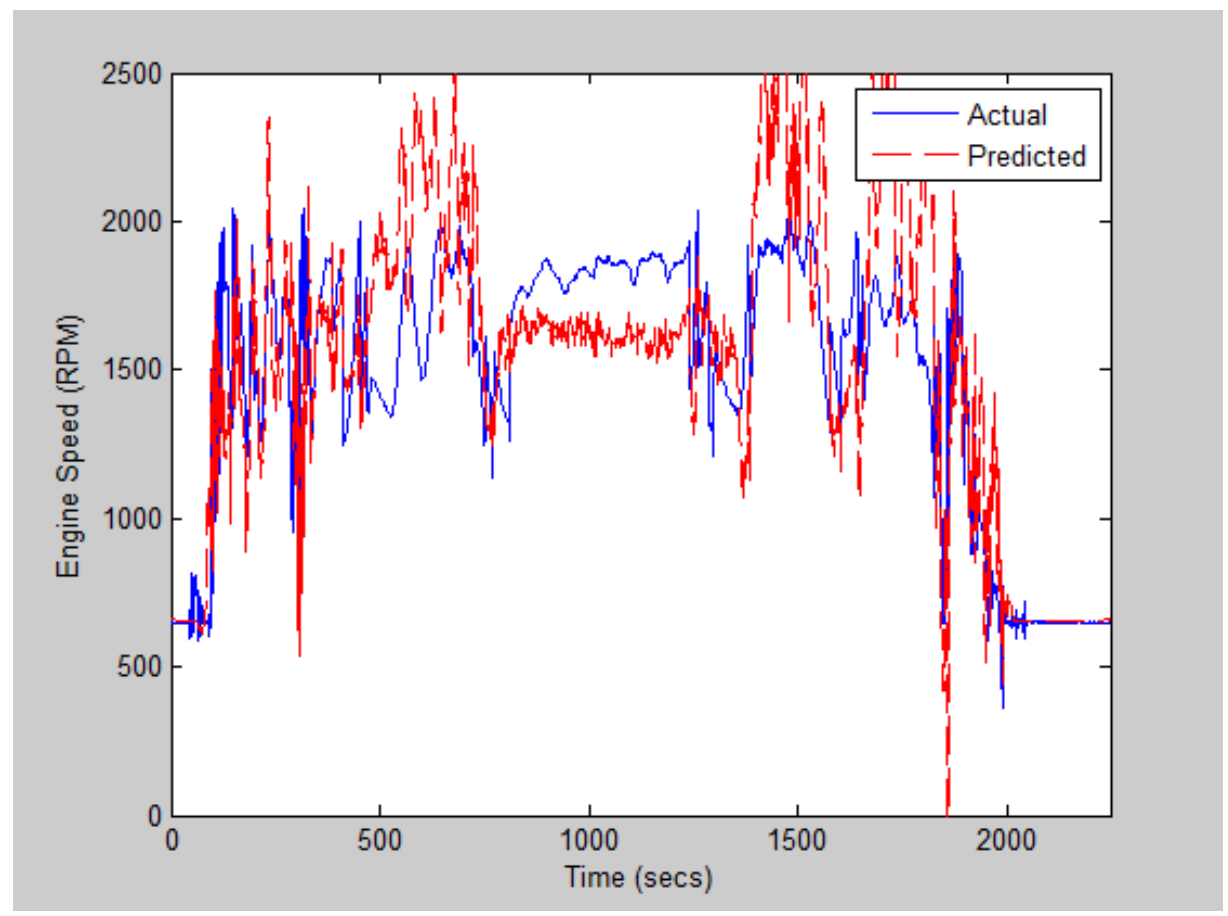

Figure 6.3.1: Difference Between Predicted and Measured Engine Speed for the Sabraton, WV to Bruceton Mills, WV Route When ANN was Trained with Washington, PA2 1

Figure 6.3.3 shows the difference between the power calculated from measured values and predicted values. It was shown in the region between 800 and 1300 seconds where the engine speed and torque were under and over predicted, respectively, that the predicted power was still closely predicted. 


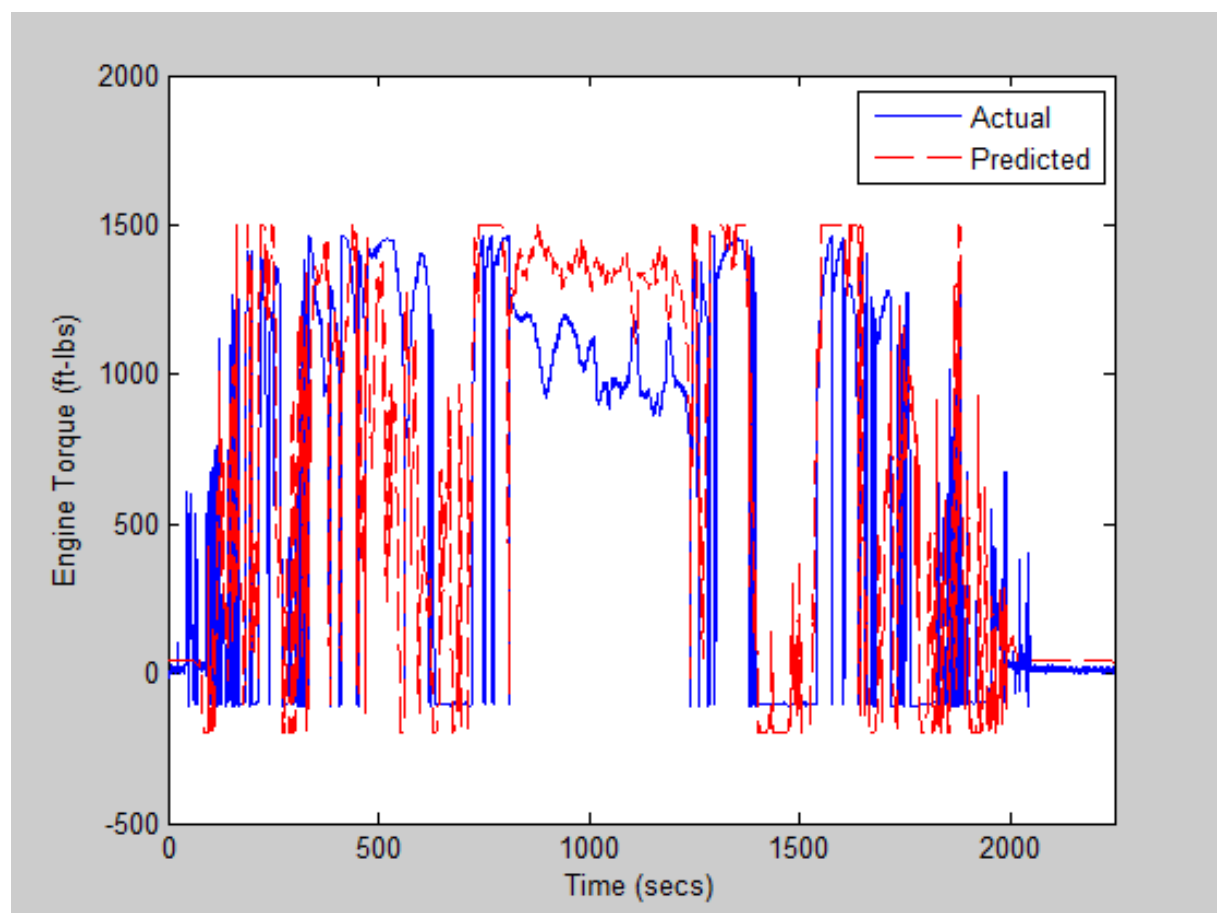

Figure 6.3.2: Difference Between Predicted and Measured Engine Torque for the Sabraton, WV to Bruceton Mills, WV Route When ANN was Trained with Washington, PA2 1

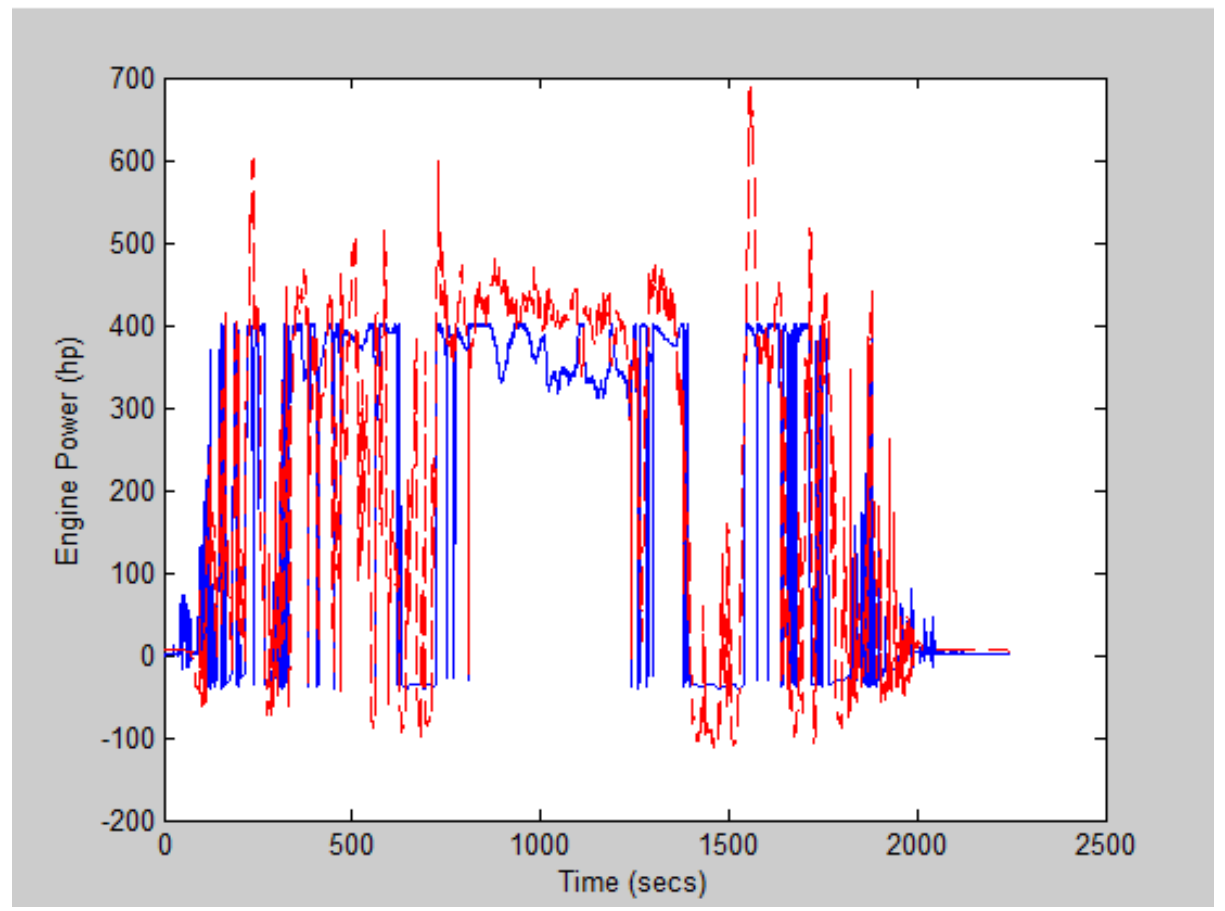

Figure 6.3.3: Difference Between Predicted and Measured Power for the Sabraton, WV to Bruceton Mills, WV Route When ANN was Trained with Washington, PA2 1 
Figure 6.3.3 shows the difference between the predicted NOx values and the measured NOx values, while Figure 6.3.4 shows the difference between the predicted $\mathrm{CO}_{2}$ and the measured $\mathrm{CO}_{2}$. It was determined that when the emissions were integrated over the entire cycle that percent differences for $\mathrm{NOx}$ and $\mathrm{CO}_{2}$ were as low as $0.3 \%$ and $14.9 \%$, respectively. It is shown that the ANN is least accurate when predicting emissions during periods where the vehicle is idling, this is demonstrated between 1400 and 1600 seconds.

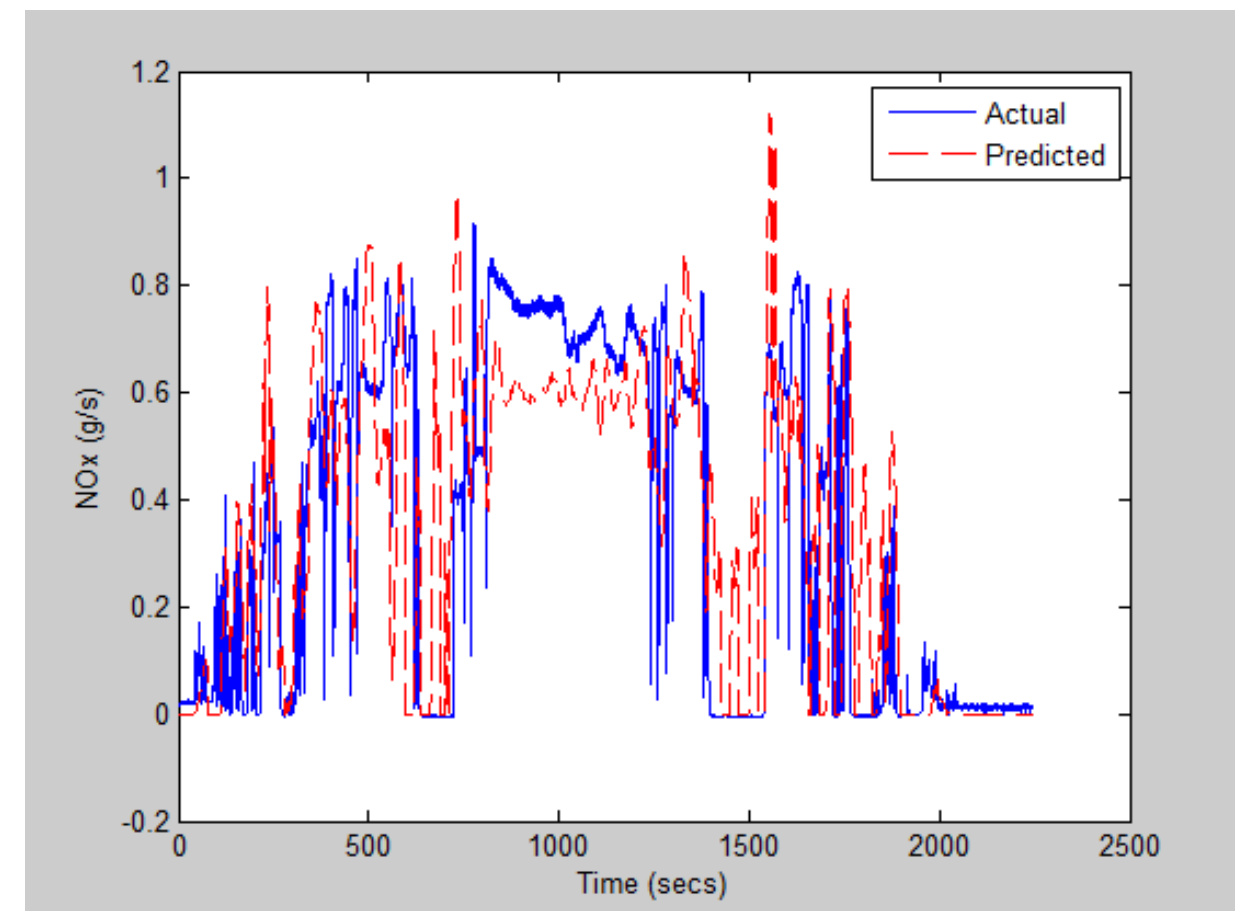

Figure 6.3.4: Difference Between Predicted and Measured NOx Emissions for the Sabraton, WV to Bruceton Mills, WV Route When ANN was Trained with Washington, PA2 1 


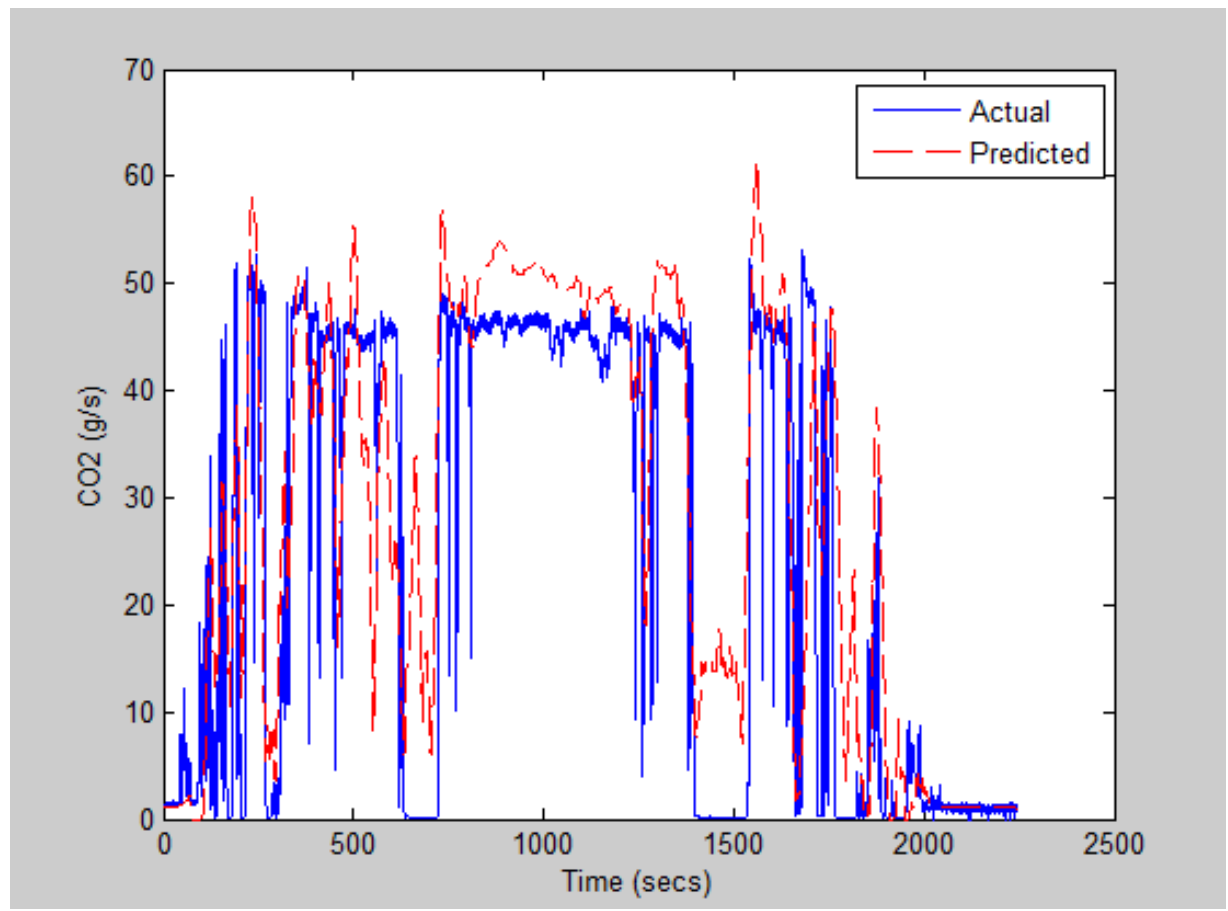

Figure 6.3.5: Difference Between Predicted and Measured $\mathrm{CO}_{2}$ Emissions for the Sabraton, WV to Bruceton Mills, WV Route When ANN was Trained with Washington, PA2 1

The data displayed in the above tables were predicted with the ANN with the emissions module trained with data from the cycle developed to simulated the Bruceton Mills, WV route. The same verification tests discussed above were also performed with the ANN when the emissions module was trained with data from the cycle developed to simulate the Washington, PA route. The results from this training combination are discussed beginning in Table 6.3.2. It was determined that a percent difference between predicted and measured integrated emissions as low as $4.4 \%$ for NOx and $12.2 \%$ for $\mathrm{CO}_{2}$ was achieved when a Bruceton Mills, WV route and the Washington, PA cycle were used for training. The ANN trained with the above mentioned combination, most accurately predicted the emissions for the route from Sabraton, WV to Bruceton Mills, WV. The average difference between the measured and predicted emissions was the lowest for the route that started in Sabraton, WV and ended in Bruceton Mills, WV. The ANN was able to predict the emissions for both the route from Sabraton, WV to Bruceton Mills, WV and the return route from Bruceton Mills, WV to Sabraton, WV with average percent differences for NOx and $\mathrm{CO}_{2}$ of less than 20\%. This demonstrated that the ANN was capable of predicts emissions for a route that differed from the route and cycles that were used for training. 
Table 6.3.2: Verification of ANN Trained with Washington, PA, and Used for Prediction of Bruceton Mills, WV Routes

\begin{tabular}{|c|c|c|c|}
\hline \multicolumn{4}{|c|}{ ANN 1 Trained with Wash PA2 1 Data } \\
\hline \multicolumn{3}{|c|}{ ANN 2 Trained with Manufacturer A PA1 Data } \\
\hline Verification Data & Run & \% Diff. NOx & \% Diff. CO 2 \\
\hline \multirow{4}{*}{ Bruceton2Sab 2 } & 1 & 24.6 & 12.4 \\
\cline { 2 - 4 } & 2 & 8.9 & 13.4 \\
\cline { 2 - 4 } & 3 & 4.7 & 18.2 \\
\cline { 2 - 4 } & Average & 12.7 & 14.6 \\
\hline \multirow{4}{*}{ Sab2Bruceton 1 } & 1 & 3.4 & 16.1 \\
\cline { 2 - 4 } & 2 & 4.4 & 17.5 \\
\cline { 2 - 4 } & 3 & 8.1 & 12.2 \\
\cline { 2 - 4 } & Average & 5.3 & 15.3 \\
\hline \multirow{5}{*}{ Bruceton2Sab 1 } & 1 & 17.0 & 19.4 \\
\cline { 2 - 4 } & 2 & 13.7 & 14.4 \\
\cline { 2 - 4 } & 3 & 17.6 & 15.8 \\
\cline { 2 - 4 } & Average & 16.1 & 16.5 \\
\hline
\end{tabular}

*The highlighted values are shown in the following figures

Figures 6.3.5 and 6.3.6 show the predicted and measured speed and torque for the route from Bruceton Mills, WV to Sabraton, WV, and correspond to the highlighted row in the above table. The largest discrepancy between the predicted and measured torque values occurred at low torques, as show between 1100 and 1300 seconds. 


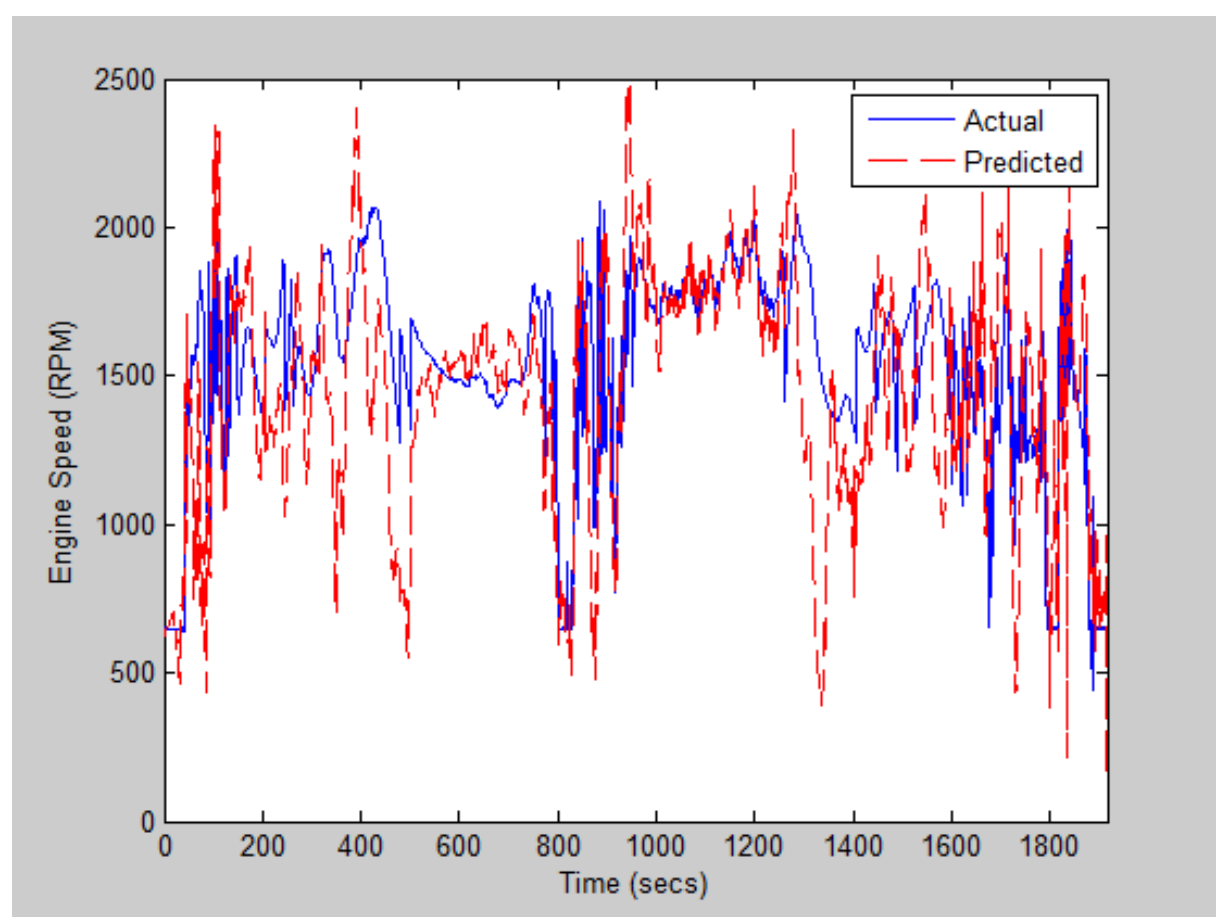

Figure 6.3.6: Difference Between Predicted and Measured Engine Speed for the Bruceton2Sab 2 Data Set

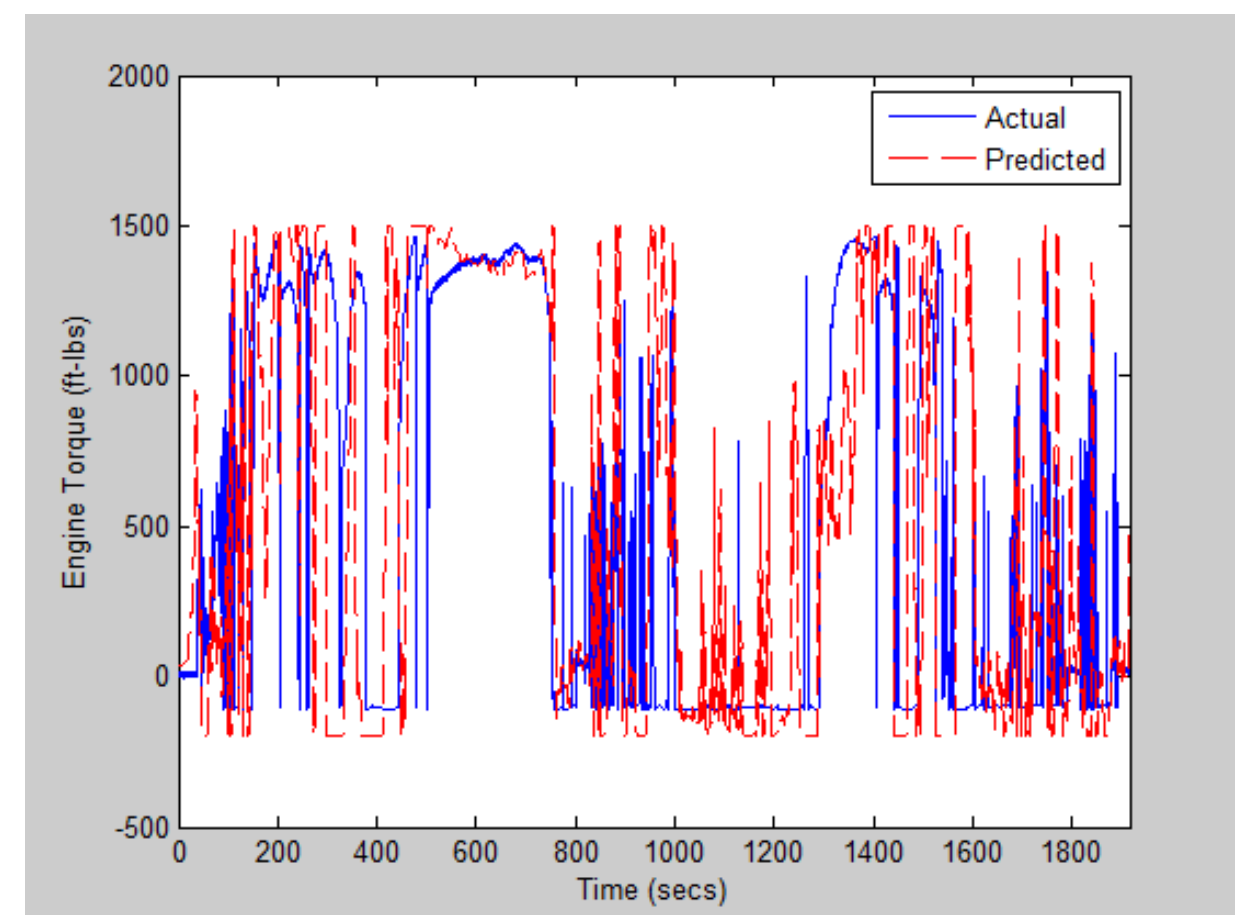

Figure 6.3.7: Difference Between Predicted and Measured Engine Torque for the Bruceton2Sab 2 Data Set 


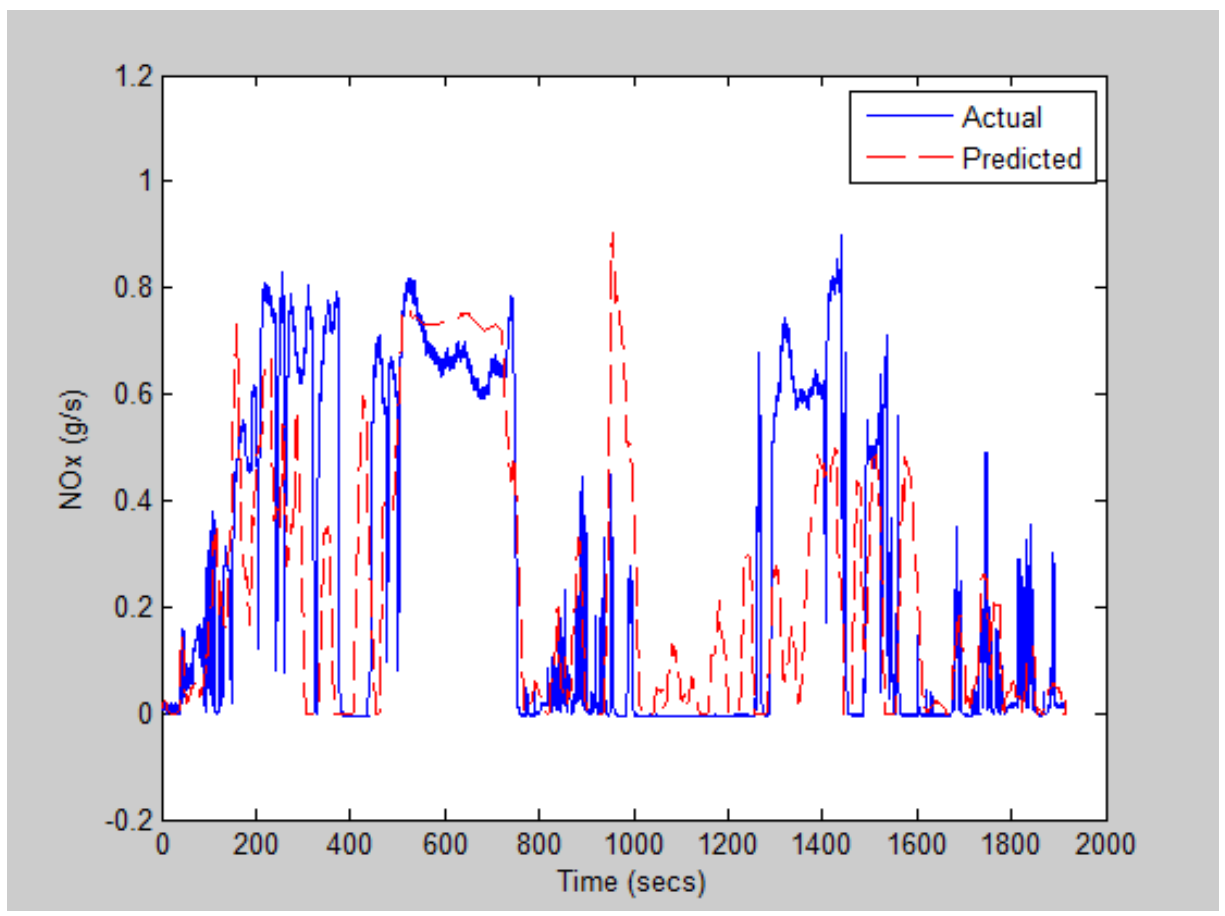

Figure 6.3.8: Difference Between Predicted and Measured NOx Emissions for the Bruceton2Sab 2 Data Set

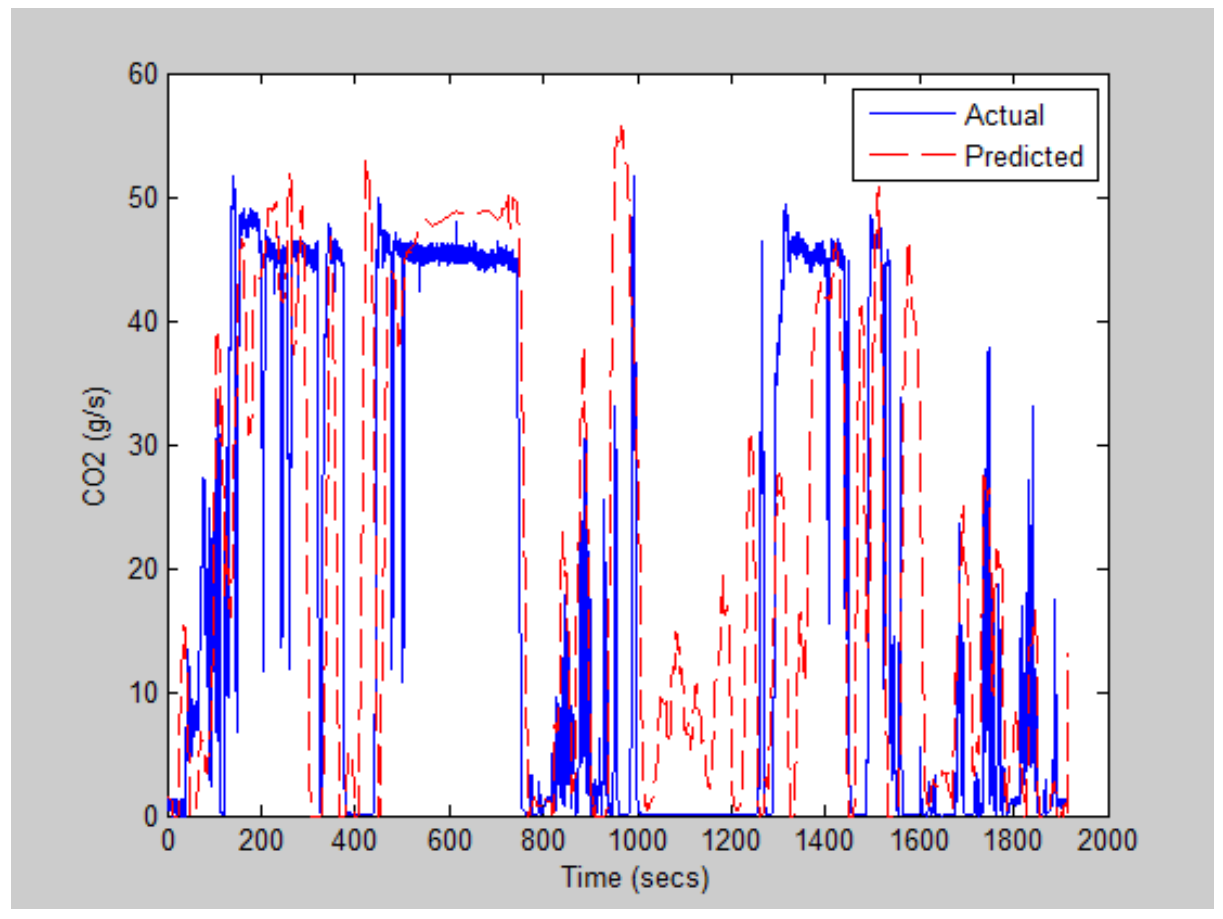

Figure 6.3.9: Difference Between Predicted and Measured $\mathrm{CO}_{2}$ Emissions for the Bruceton2Sab 2 Data Set 


\subsection{Training and Predicting with Same Route, and Trained with Different Cycle}

In this section the results were obtained when the vehicle ANN was trained with in-use data from the Bruceton Mills, WV to Sabraton, WV route and the emissions ANN was trained with engine dynamometer data from the Washington, PA 1 cycle. Two data sets from the Sabraton, WV to Bruceton Mills, WV route, and one data set from the Bruceton Mills, WV to Sabraton, WV route were used to verify the accuracy of the ANN model. Table 6.4.1 displays the percent differences between the measured and the predicted emissions for the routes examined. It was determined for the above mentioned training scenario that the ANN was able to predict the emissions associated with the Sabraton, WV to Bruceton, WV route with average percent differences less than $14 \%$ for $\mathrm{NOx}$ and $16 \%$ for $\mathrm{CO}_{2}$.

Table 6.4.1: Verification of ANN Trained With Bruceton Mills, WV Data, and Used for Prediction of Bruceton Mills, WV Routes

\begin{tabular}{|c|c|c|c|}
\hline \multicolumn{4}{|c|}{ ANN 1 Trained with Bruceton2Sab 2 Route Data } \\
\hline \multicolumn{3}{|c|}{ ANN 2 Trained with Manufacturer A PA1 Cycle Data } \\
\hline Verification Data & Run & \% Diff. NOx & \% Diff. CO \\
\hline \multirow{4}{*}{ Sab2Bruceton 1 } & 1 & 7.7 & 12.8 \\
\cline { 2 - 4 } & 2 & 15.6 & 14.3 \\
\cline { 2 - 4 } & 3 & 3.5 & 19.8 \\
\cline { 2 - 4 } & Average & 8.9 & 15.6 \\
\hline \multirow{4}{*}{ Sab2Bruceton 2 } & 1 & 21.0 & 4.1 \\
\cline { 2 - 4 } & 2 & 6.7 & 17.5 \\
\cline { 2 - 4 } & 3 & 13.8 & 15.0 \\
\cline { 2 - 4 } & Average & 13.8 & 12.2 \\
\hline \multirow{5}{*}{ Bruceton2Sab 1 } & 1 & 5.1 & 27.0 \\
\cline { 2 - 4 } & 2 & 11.9 & 29.1 \\
\cline { 2 - 4 } & 3 & 2.9 & 28.3 \\
\cline { 2 - 4 } & Average & 6.6 & 28.1 \\
\hline
\end{tabular}

*The highlighted values are shown in the following figures

Figures 6.4.1 through 6.4.4 compare the predicted values to the measured values for first verification run with the Sab2Bruceton 1 data set, which is highlighted in the above table. Figure 6.4.1 compares the predicted engine speed to the measured engine speed, while Figure 6.4.2 
compares the predicted engine torque to the measured engine torque. As shown previously, between 700 and 1300 seconds the ANN predicts the speed lower than the actual value, and the torque higher than the actual value, indicating it is predicting the incorrect gear, but similar engine power.

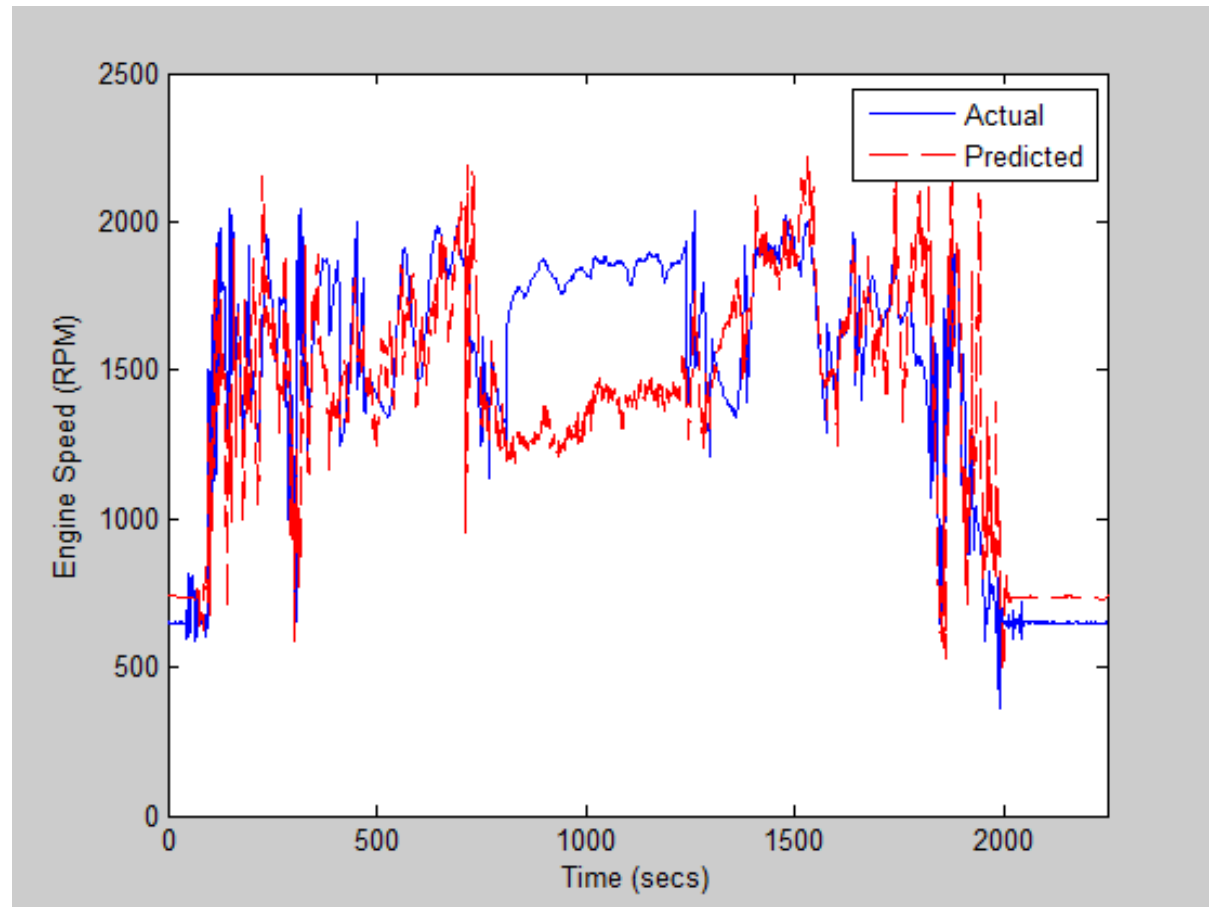

Figure 6.4.1: Difference Between Predicted and Measured Engine Speed for the Sab2Bruceton 1 Data Set 


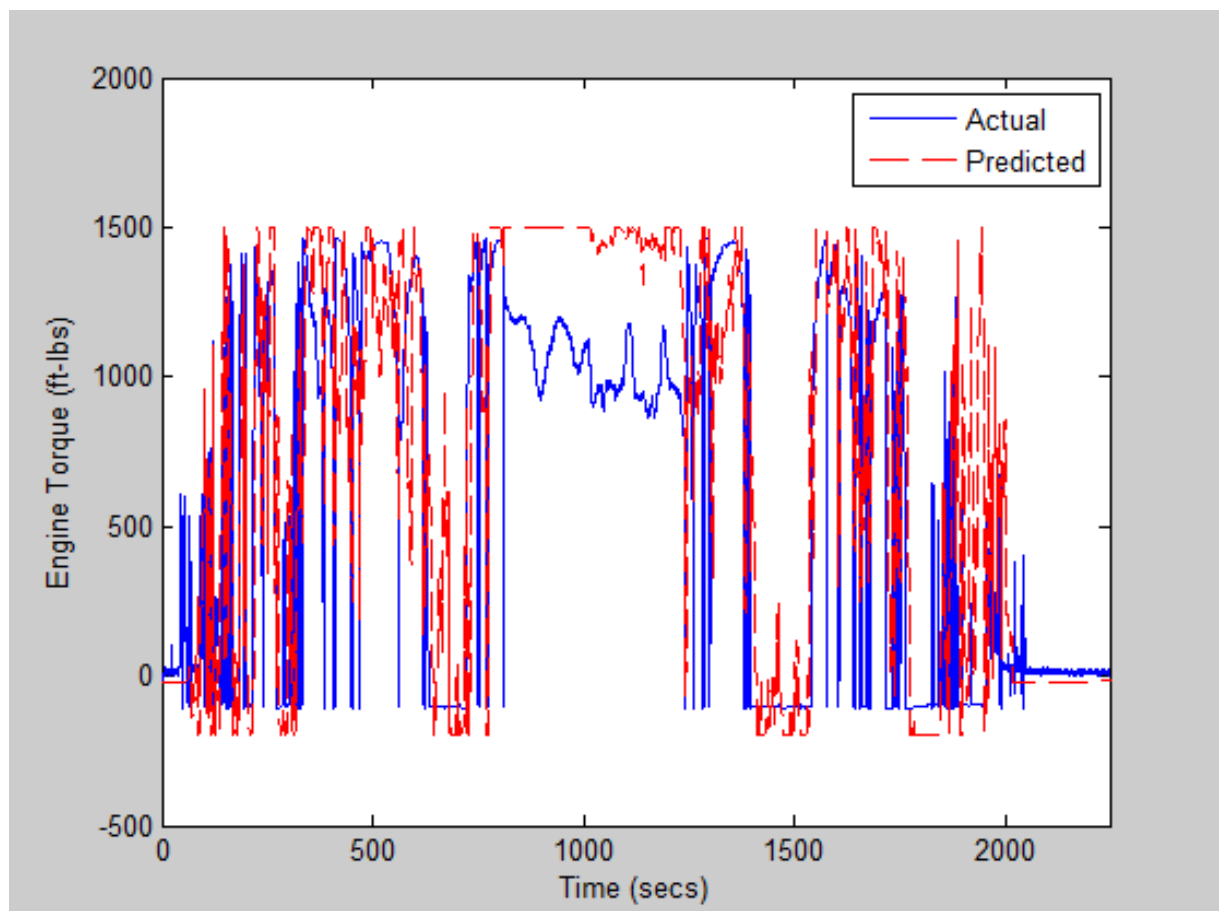

Figure 6.4.2: Difference Between Predicted and Measured Engine Torque for the Sab2Bruceton 1 Data Set

Comparisons of the predicted and measured emissions are shown in Figures 6.4.3 for NOx and 6.4.4 for $\mathrm{CO}_{2}$. The ANN prediction for NOx is below that measured value between 700 and 1000 seconds, however the integrated percent difference between the measured and predicted NOx emissions was $7.7 \%$. The ANN predicted $\mathrm{CO}_{2}$ emissions with a percent difference from the measured values of $12.8 \%$. 


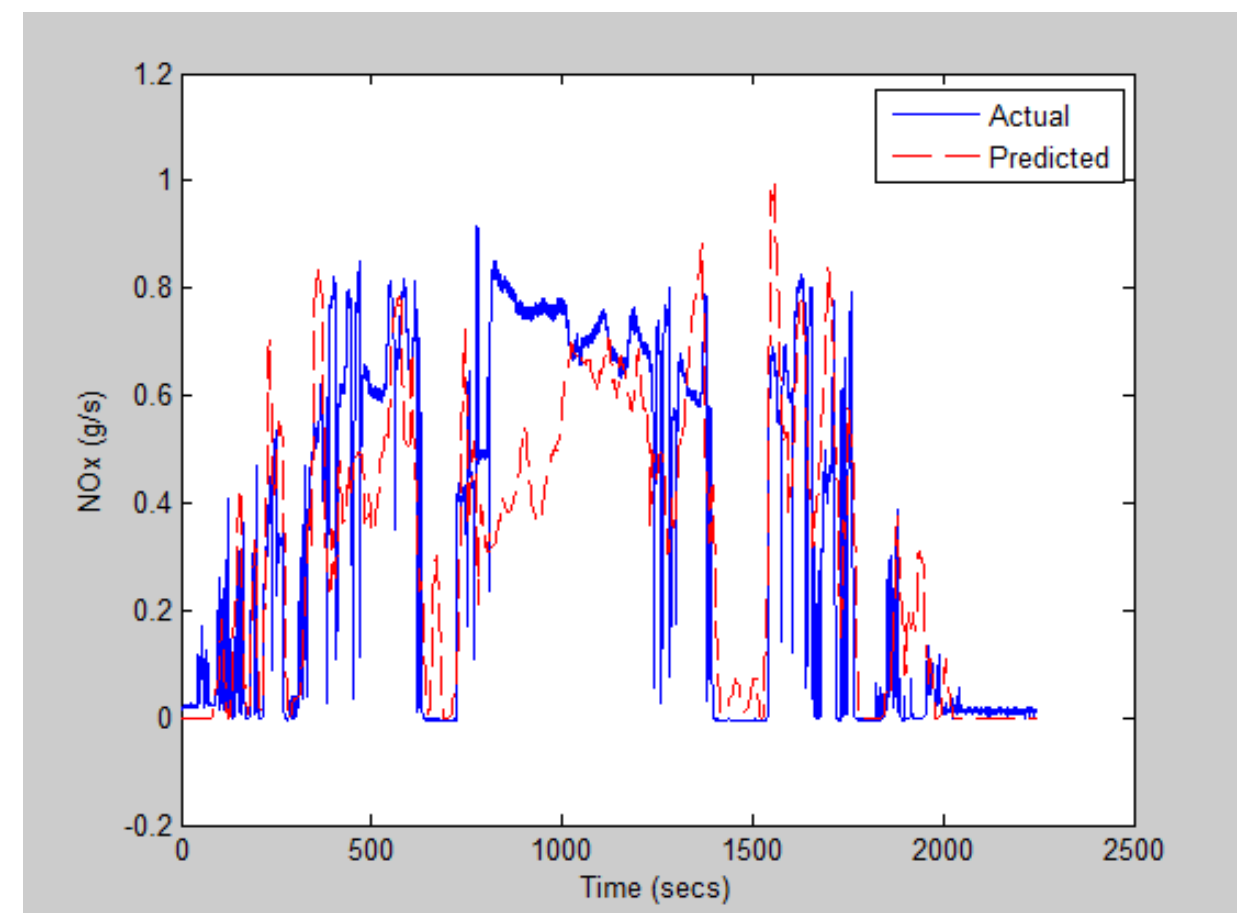

Figure 6.4.3: Difference Between Predicted and Measured NOx Emissions for the Sab2Bruceton 1 Data Set

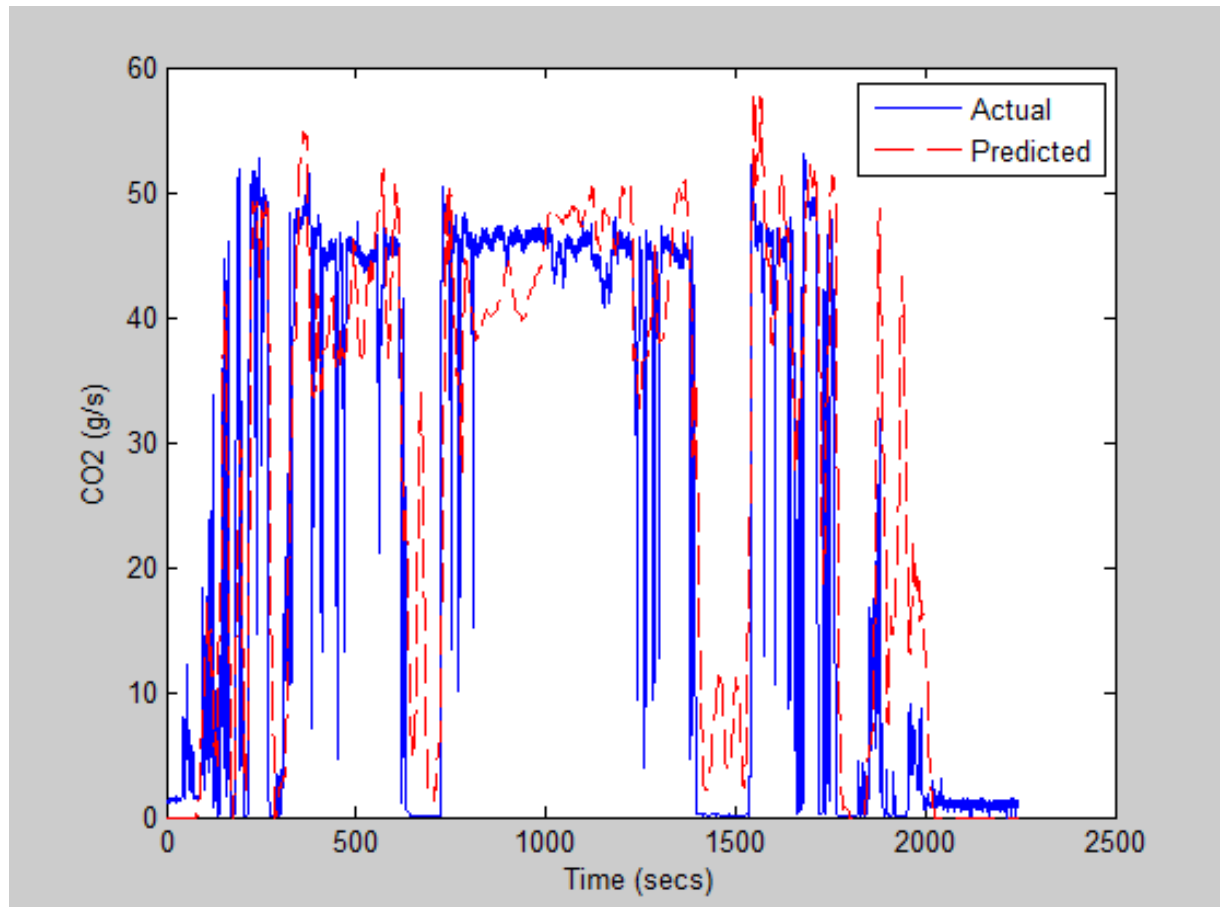

Figure 6.4.4: Difference Between Predicted and Measured $\mathrm{CO}_{2}$ Emissions for the Sab2Bruceton 1 Data Set 
Table 6.4.2 displays the percent differences between the measured and predicted integrated emissions for various runs of the Washington, PA routes. The previously presented emissions predictions for the Washington, PA routes were conducted when the emissions module of the ANN was trained with data from the Washington, PA 1 cycle. The data in the table below was acquired for scenarios where the vehicle module of the ANN was trained with in-use data from the Washington, PA 2 route, and the emissions module of the ANN was trained with data from the Sabraton, WV to Bruceton Mills, WV dynamometer cycle. The ANN was employed to predict emissions associated with two separate runs of the Washington, PA 1 route, and one run of the Washington, PA 2 route.

Table 6.4.2: Verification of ANN Trained with Washington, PA Data and Used for Prediction of Washington, PA Routes

\begin{tabular}{|c|c|c|c|}
\hline \multicolumn{4}{|c|}{ ANN 1 Trained with Wash PA2 1 Route Data } \\
\hline ANN 2 Trained with Manufacturer A Sabraton to Bruceton Cycle Data \\
\hline Verification Data & Run & \% Diff. NOx & \% Diff. CO 2 \\
\hline \multirow{4}{*}{ Wash PA1 2 } & 1 & 24.0 & 13.1 \\
\cline { 2 - 4 } & 2 & 26.8 & 16.0 \\
\cline { 2 - 4 } & 3 & 16.3 & 14.0 \\
\cline { 2 - 4 } & Average & 22.4 & 14.3 \\
\hline \multirow{4}{*}{ Wash PA2 2 } & 1 & 16.7 & 1.9 \\
\cline { 2 - 4 } & 2 & 24.6 & 1.9 \\
\cline { 2 - 4 } & 3 & 28.9 & 7.2 \\
\cline { 2 - 4 } & Average & 23.4 & 3.6 \\
\hline \multirow{5}{*}{ Wash PA1 1 } & 1 & 6.54 & 5.6 \\
\cline { 2 - 4 } & 2 & 10.0 & 3.4 \\
\cline { 2 - 4 } & 3 & 4.5 & 4.5 \\
\cline { 2 - 4 } & Average & 5.8 & 3.4 \\
\hline
\end{tabular}

*The highlighted values are shown in the following figures

Figure 6.4.5 shows the difference between predicted engine speed and the measured engine speed, while Figure 6.4.6 shows the difference between the predicted engine torque and the measured engine torque. For this verification run, the ANN was trained with data set Washington PA2 1 and verification was performed with data set Washington PA1 1. The ANN 
was again shown to under-estimate the engine speed during idle conditions and predicted torque values below those experienced in both the training and verification data.

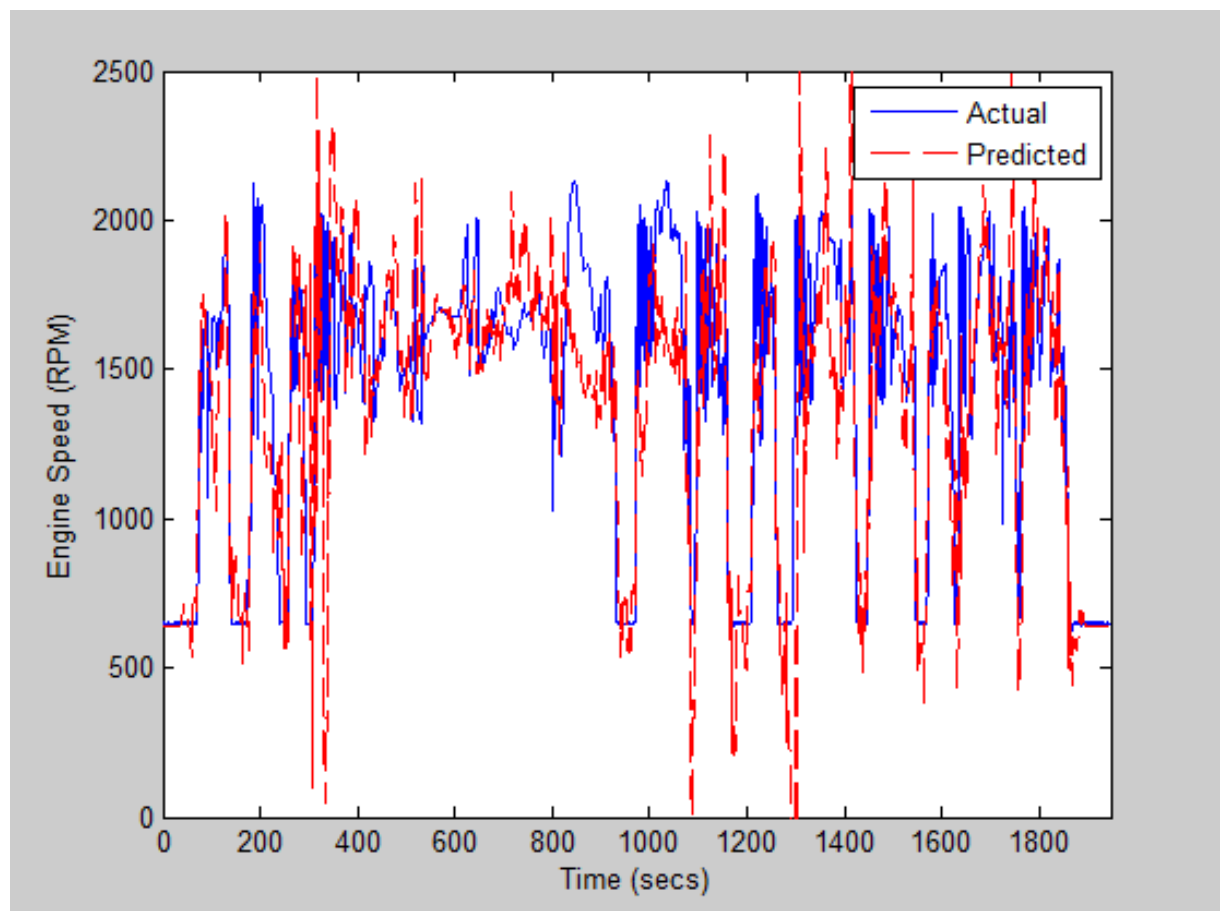

Figure 6.4.5: Difference Between Predicted and Measured Engine Speed for the Washington, PA 1 Route When Trained with Washington, PA2 1 


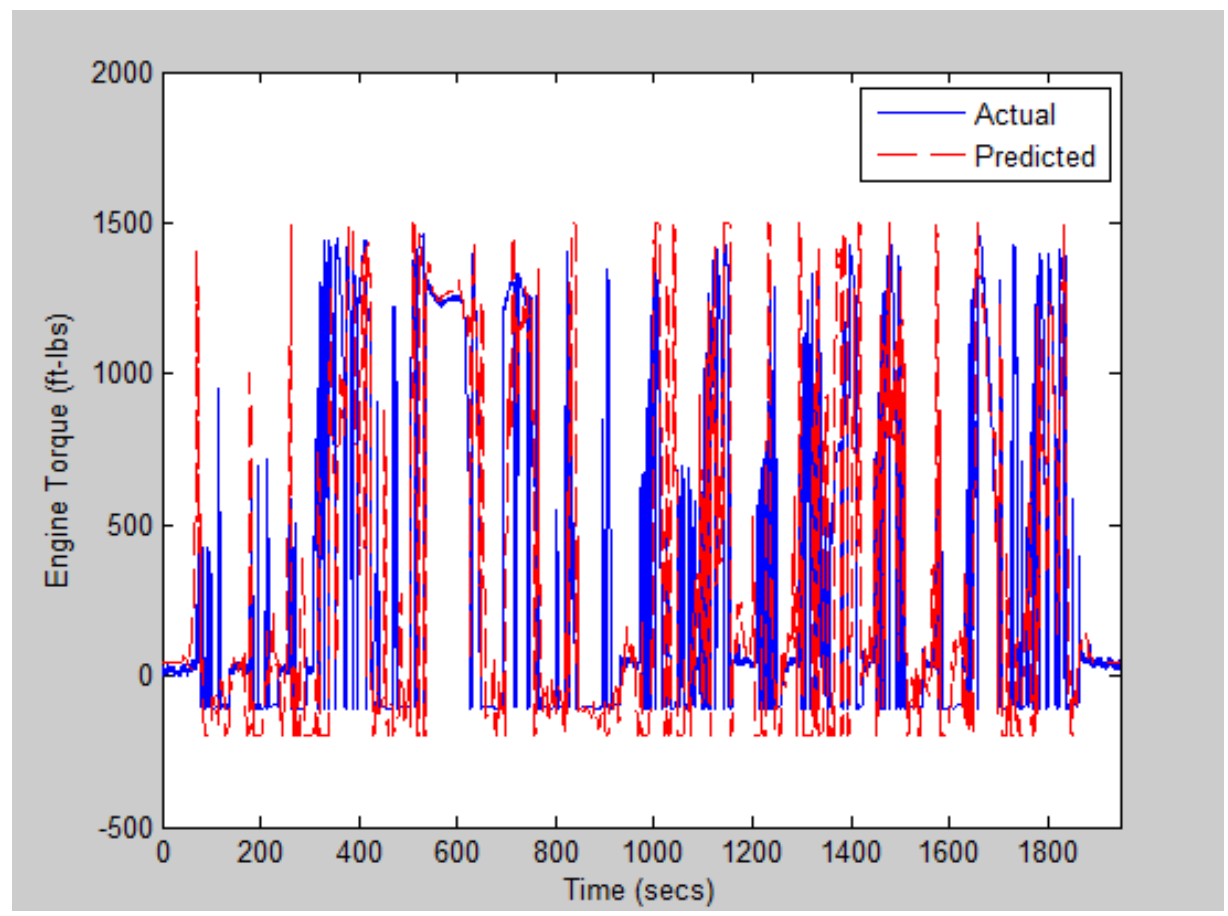

Figure 6.4.6: Difference Between Predicted and Measured Torque for the Washington, PA 1 Route When Trained with Washington, PA2 1

Figure 6.4.7 shows the difference between the predicted $\mathrm{CO}_{2}$ values and the measured $\mathrm{CO}_{2}$ values, while Figure 6.4.8 shows the difference between the predicted NOx and the measured NOx. It was determined that when the emissions were integrated over the entire cycle that the percent differences for $\mathrm{NOx}$ and $\mathrm{CO}_{2}$ were $4.5 \%$ and $4.5 \%$, respectively. The predicted $\mathrm{CO}_{2}$ emissions more closely followed the trend of the measured emissions, with less over-shoot than the predicted NOx values. 


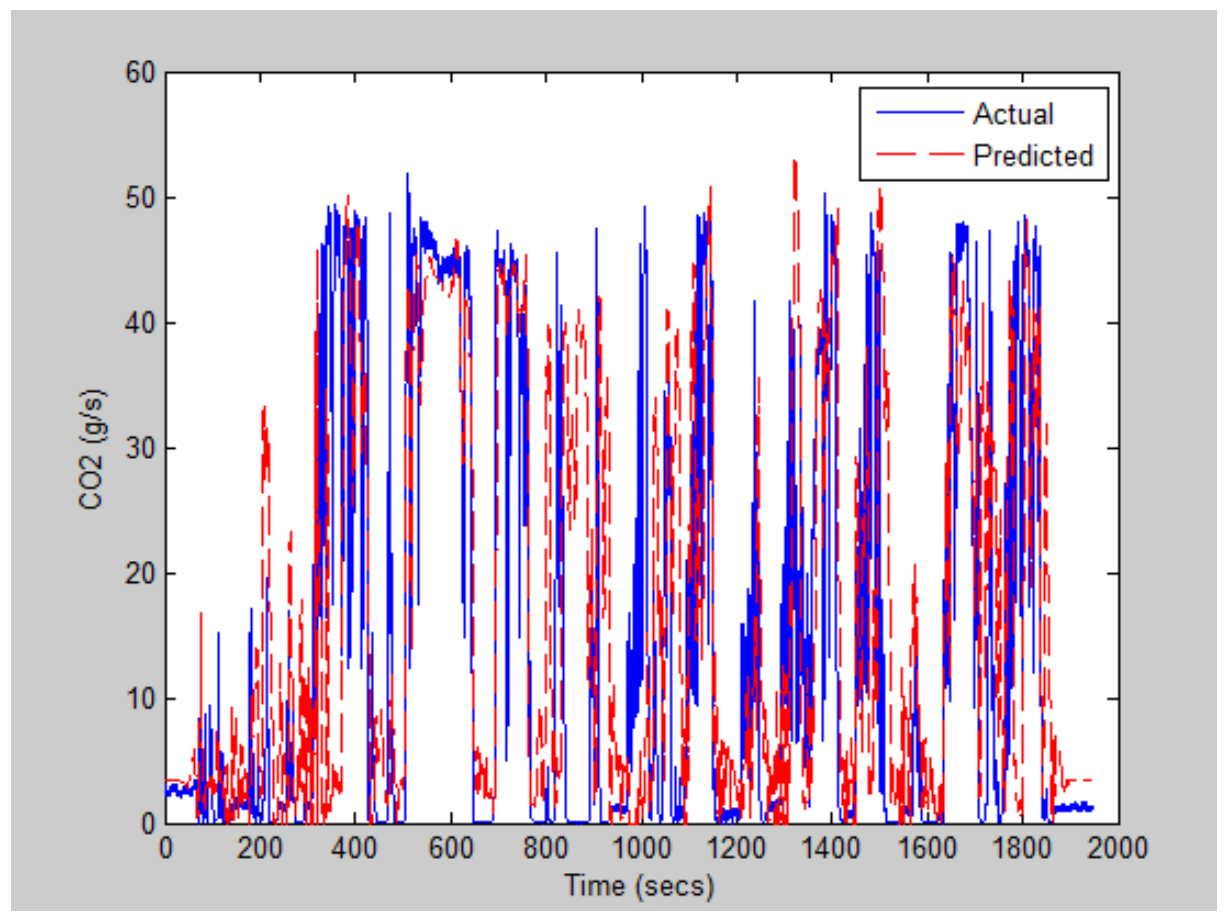

Figure 6.4.7: Difference Between Predicted and Measured $\mathrm{CO}_{2}$ for the Washington, PA 1 Route When Trained with Washington, PA2 1

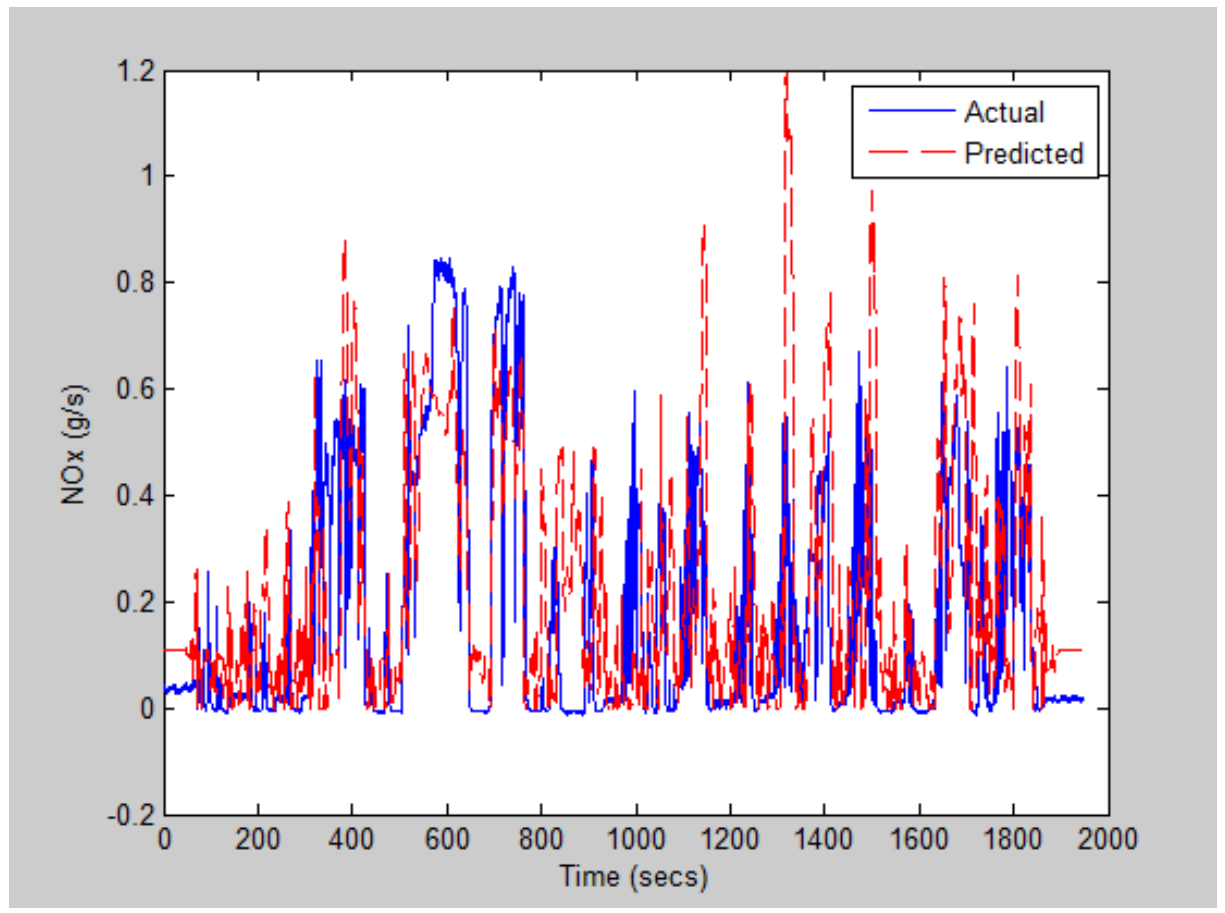

Figure 6.4.8: Difference Between Predicted and Measured NOx for the Washington, PA 1 Route When Trained with Washington, PA2 1 


\subsection{Comparison of Measured and Predicted Emissions to EPA Regulations}

Tables 6.5.1 and 6.5.2 show comparisons between the measured emissions values, predicted emissions values, and the EPA regulations associated with the model year of the engine being modeled. This comparison was conducted to relate the predicted and measured emissions to accepted standards, in order to demonstrate that the ANN was capable of predicting realistic values for NOx emissions. The EPA does not regulate the quantity of $\mathrm{CO}_{2}$ emitted from vehicles, therefore only the NOx emissions were compared to the EPA regulations. The engine being modeled was a 1995 Manufacturer A 400 hp engine, for which the EPA limited the NOx production to $5.0 \mathrm{~g} / \mathrm{bhp}-\mathrm{hr}$. It should be noted that the emissions discussed in this section are on a brake-specific mass (g/bhp-hr) basis, in previous section the emissions were compared on a mass rate $(\mathrm{g} / \mathrm{s})$ basis. The predicted power was used in calculations for the predicted values, and the measured power was used for calculations with the measured emissions. The data in Tables 6.5.1 and 6.5.2 was obtained when the emissions module of the ANN was trained with data from the engine dynamometer cycle designed to simulate the Washington, PA route and the vehicle module of the ANN was trained with data for in-use testing on the Bruceton Mills, WV route. Emissions for two different runs of the Bruceton Mills, WV route were then predicted with the trained model.

It was determined that for the data set Sab2Bruceton 1 the quantities of $\mathrm{NOx}$ and $\mathrm{CO}_{2}$ produced were $6.6 \mathrm{~g} / \mathrm{bhp}-\mathrm{hr}$ and $465.0 \mathrm{~g} / \mathrm{bhp}-\mathrm{hr}$, respectively. The average emissions values associated with the three runs of the ANN were $5.5 \mathrm{~g} / \mathrm{bhp}-\mathrm{hr}$ for $\mathrm{NOx}$ and $489.3 \mathrm{~g} / \mathrm{bhp}-\mathrm{hr}$ for $\mathrm{CO}_{2}$. The percent differences between the predicted and actual $\mathrm{NOx}$ and $\mathrm{CO}_{2}$ were $16.9 \%$ and $5.2 \%$. The percent difference between the EPA regulation for NOx and the measured NOx emissions was $32.2 \%$, while the difference between the predicted NOx emissions and the EPA regulation was $10.3 \%$. The difference between the predicted emissions, measured emissions, and the EPA regulation for NOx for data set Sab2Bruceton 2 are also displayed in the table, and were determined to be similar to the values associated with data set Sab2Bruceton 1. 
Table 6.5.1: Predicted Values, Measured Values, and EPA Regulations for ANN Trained with Bruceton2Sab 1 and Washington, PA1 Engine Dynamometer Data

\begin{tabular}{|c|c|c|c|c|c|c|}
\hline \multicolumn{7}{|c|}{ ANN 1 Trained with Bruceton2Sab 1 Data } \\
\hline \multicolumn{7}{|c|}{ ANN 2 Trained with Manufacturer A PA1 Data } \\
\hline \multirow[t]{2}{*}{ Verification Data } & \multirow[t]{2}{*}{ Run } & \multicolumn{2}{|c|}{$\begin{array}{l}\text { Measured } \\
\text { (g/bhp-hr) }\end{array}$} & \multicolumn{2}{|c|}{$\begin{array}{l}\text { Predicted } \\
\text { (g/bhp-hr) }\end{array}$} & \multirow{2}{*}{$\frac{\text { EPA Reg. }(g / b h p-h r)}{\text { NOx }}$} \\
\hline & & NOx & $\mathrm{CO}_{2}$ & NOx & $\mathrm{CO}_{2}$ & \\
\hline \multirow{4}{*}{ Sab2Bruceton 1} & 1 & 6.6 & 465 & 5.2 & 485 & 5.0 \\
\hline & 2 & 6.6 & 465 & 6.1 & 493 & 5.0 \\
\hline & 3 & 6.6 & 465 & 5.3 & 490 & 5.0 \\
\hline & Average & 6.6 & 465 & 5.5 & 489 & 5.0 \\
\hline \multirow{4}{*}{ Sab2Bruceton 2} & 1 & 6.5 & 456 & 4.7 & 436 & 5.0 \\
\hline & 2 & 6.5 & 456 & 5.6 & 493 & 5.0 \\
\hline & 3 & 6.5 & 456 & 6.8 & 482 & 5.0 \\
\hline & Average & 6.5 & 456 & 5.7 & 470 & 5.0 \\
\hline
\end{tabular}

Table 6.5.2: Comparison of Predicted Values and Measured Values to EPA Regulations for

ANN Trained with Bruceton2Sab 1 and Washington, PA1 Engine Dynamometer Data

\begin{tabular}{|c|c|c|c|c|c|}
\hline \multicolumn{7}{|c|}{ ANN 1 Trained with Bruceton2Sab 1 Data } \\
\hline \multirow{3}{*}{ Verification Data } & Run & $\begin{array}{c}\text { \% Diff. Meas. } \\
\text { NOx vs. EPA }\end{array}$ & $\begin{array}{c}\text { \% Diff. Pred. } \\
\text { NOx vs. EPA }\end{array}$ & $\begin{array}{c}\text { \% Diff. Meas. } \\
\text { NOx vs. Pred. } \\
\text { NOx }\end{array}$ & $\begin{array}{c}\text { \% Diff. Meas. } \\
\mathrm{CO}_{2} \text { vs. Pred. } \\
\text { CO }_{2}\end{array}$ \\
\hline \multirow{3}{*}{ Sab2Bruceton 1 } & 1 & 32.8 & 3.4 & 22.1 & 4.3 \\
\cline { 2 - 6 } & 2 & 32.8 & 21.8 & 8.3 & 6.0 \\
\cline { 2 - 6 } & 3 & 32.8 & 5.8 & 20.3 & 5.4 \\
\cline { 2 - 6 } & Average & 32.8 & 10.3 & 16.9 & 5.2 \\
\cline { 2 - 6 } & 1 & 30.2 & 5.4 & 27.3 & 4.3 \\
\cline { 2 - 6 } & 2 & 30.2 & 11.6 & 14.3 & 8.0 \\
\cline { 2 - 6 } & 3 & 30.2 & 36.4 & 4.8 & 5.8 \\
\cline { 2 - 6 } & Average & 30.2 & 17.8 & 15.5 & 6.0 \\
\hline
\end{tabular}

Tables 6.5.3 and 6.5.4 show comparisons between the measured emissions values, predicted emissions values, and the EPA regulations associated with the model year of the engine being modeled. The data in Tables 6.5.3 and 6.5.4 was obtained when the emissions module of the ANN was trained with data from the engine dynamometer cycle designed to simulate the Bruceton Mills, WV route and the vehicle module of the ANN was trained with data for in-use 
testing on the Washington, PA route. Emissions for two different runs of the Bruceton Mills, WV route were then predicted with the trained model.

It was determined that for the data set Sab2Bruceton 1 the quantities of $\mathrm{NOx}$ and $\mathrm{CO}_{2}$ produced were $6.6 \mathrm{~g} / \mathrm{bhp}-\mathrm{hr}$ and $465.0 \mathrm{~g} / \mathrm{bhp}-\mathrm{hr}$, respectively. The average emissions values associated with the three runs of the ANN were $5.7 \mathrm{~g} / \mathrm{bhp}-\mathrm{hr}$ for NOx and $461.1 \mathrm{~g} / \mathrm{bhp}-\mathrm{hr}$ for $\mathrm{CO}_{2}$. The percent differences between the predicted and measured $\mathrm{NOx}$ and $\mathrm{CO}_{2}$ were $14.7 \%$ and $2.1 \%$. The percent difference between the EPA regulation for NOx and the measured NOx emissions was $32.8 \%$, while the difference between the predicted NOx emissions and the EPA regulation was $17.7 \%$. The difference between the predicted emissions, measured emissions, and the EPA regulation for NOx for data set Bruceton2Sab 1 are also displayed in the table. It was determined that the brake-specific emissions were most closely predicted for data set Bruceton2Sab 1, when the ANN was trained with Washington, PA route in-use data and Bruceton Mills, WV cycle engine dynamometer data. The percent difference between measured and predicted $\mathrm{NOx}$ and $\mathrm{CO}_{2}$ were $5.9 \%$ and $1.1 \%$, respectively.

Table 6.5.3: Predicted Values, Measured Values, and EPA Regulations for ANN Trained with Washington, PA2 1 and Sabraton to Bruceton Engine Dynamometer Data

\begin{tabular}{|c|c|c|c|c|c|c|}
\hline \multicolumn{7}{|c|}{ ANN 1 Trained with Wash PA2 1 Data } \\
\hline \multicolumn{7}{|c|}{ ANN 2 Trained with Manufacturer A Sabraton to Bruceton Data } \\
\hline \multirow{2}{*}{ Verification Data } & \multirow{2}{*}{ Run } & \multicolumn{2}{|c|}{$\begin{array}{l}\text { Measured } \\
\text { (g/bhp-hr) }\end{array}$} & \multicolumn{2}{|c|}{$\begin{array}{l}\text { Predicted } \\
\text { (g/bhp-hr) }\end{array}$} & \multirow{2}{*}{$\frac{\text { EPA Reg. }(g / b h p-h r)}{\text { NOx }}$} \\
\hline & & NOx & $\mathrm{CO}_{2}$ & NOx & $\mathrm{CO}_{2}$ & \\
\hline \multirow{4}{*}{ Sab2Bruceton 1} & 1 & 6.6 & 465 & 6.5 & 452 & 5.0 \\
\hline & 2 & 6.6 & 465 & 4.7 & 457 & 5.0 \\
\hline & 3 & 6.6 & 465 & 5.8 & 474 & 5.0 \\
\hline & Average & 6.6 & 465 & 5.7 & 461 & 5.0 \\
\hline \multirow{4}{*}{ Bruceton2Sab 1} & 1 & 6.4 & 456 & 6.7 & 450 & 5.0 \\
\hline & 2 & 6.4 & 456 & 6.7 & 460 & 5.0 \\
\hline & 3 & 6.4 & 456 & 5.8 & 452 & 5.0 \\
\hline & Average & 6.4 & 456 & 6.4 & 454 & 5.0 \\
\hline
\end{tabular}


Table 6.5.4: Comparison of Predicted Values and Measured Values to EPA Regulations for ANN Trained with Washington, PA2 1 and Sabraton to Bruceton Engine Dynamometer Data

\begin{tabular}{|c|c|c|c|c|c|}
\hline \multicolumn{7}{|c|}{ ANN 1 Trained with Washington, PA2 1 Data } \\
\hline \multirow{3}{*}{ Verification Data 2 Trained with Manufacturer A Sabraton to Bruceton Data } \\
\hline \multirow{5}{*}{ Sab2Bruceton 1 } & Run & $\begin{array}{c}\text { \% Diff. Meas. } \\
\text { NOx vs. EPA }\end{array}$ & $\begin{array}{c}\text { \% Diff. Pred. } \\
\text { NOx vs. EPA }\end{array}$ & $\begin{array}{c}\text { \% Diff. Meas. } \\
\text { NOx vs. Pred. } \\
\text { NOx }\end{array}$ & $\begin{array}{c}\text { \% Diff. Meas. } \\
\text { CO }_{2} \text { vs. Pred. } \\
\text { CO }_{2}\end{array}$ \\
\cline { 2 - 6 } & 1 & 32.8 & 29.8 & 2.3 & 2.7 \\
\cline { 2 - 6 } & 2 & 32.8 & 6.6 & 29.7 & 1.7 \\
\cline { 2 - 6 } & Average & 32.8 & 16.8 & 12.0 & 1.9 \\
\hline \multirow{5}{*}{ Bruceton2Sab 1 } & 1 & 28.8 & 33.6 & 14.7 & 2.1 \\
\cline { 2 - 6 } & 2 & 28.8 & 34.2 & 3.7 & 1.3 \\
\cline { 2 - 6 } & 3 & 28.8 & 16.2 & 4.2 & 0.8 \\
\cline { 2 - 6 } & Average & 28.8 & 28.0 & 9.8 & 0.9 \\
\hline
\end{tabular}

\subsection{HC and CO Predicted Values}

In the previous portions of this document the predicted $\mathrm{NOx}$ and $\mathrm{CO}_{2}$ emissions have been discussed and compared to measured values. The $\mathrm{HC}$ and $\mathrm{CO}$ emissions for each $\mathrm{ANN}$ training combination and verification set were also predicted, however in-use data was not available for measured values of $\mathrm{HC}$ and $\mathrm{CO}$, and therefore the differences between predicted values and measured values were not discussed. In order to show the accuracy of the model in predicting $\mathrm{HC}$ and $\mathrm{CO}$ emissions, the integrated predicted emissions values were compared to the integrated measured emissions values associated with engine dynamometer testing. The EPA regulations for $\mathrm{CO}$ and $\mathrm{HC}$ emissions that apply to the engine being examined were $15.5 \mathrm{~g} / \mathrm{bhp}$-hr and 1.3 $\mathrm{g} / \mathrm{bhp}-\mathrm{hr}$, respectively. The quantities of $\mathrm{HC}$ and $\mathrm{CO}$ produced by diesel engines are consistently lower than the EPA regulated values, therefore it was determined that comparing the predicted $\mathrm{HC}$ and $\mathrm{CO}$ emissions to other measured values was a more useful metric to analyze the accuracy of prediction. Tables 6.6.1 and 6.6.2 compare the predicted integrated brake-specific $\mathrm{HC}$ and $\mathrm{CO}$ emissions to the values that were measured during engine dynamometer testing employing the cycle that simulated the Bruceton Mills, WV route. The average predicted integrated emissions for $\mathrm{CO}$ and $\mathrm{HC}$ were $0.35 \mathrm{~g} / \mathrm{bhp}-\mathrm{hr}$ and $0.029 \mathrm{~g} / \mathrm{bhp}-\mathrm{hr}$, respectively. The measured emissions values associated with the engine dynamometer for $\mathrm{CO}$ and $\mathrm{HC}$ were 0.30 $\mathrm{g} / \mathrm{bhp}-\mathrm{hr}$ and $0.041 \mathrm{~g} / \mathrm{bhp}-\mathrm{hr}$, respectively. 
Table 6.6.1 Predicted and Measured Values for $\mathrm{HC}$ and $\mathrm{CO}$ for ANN Trained with Washington PA2 1 and Sabraton, WV to Bruceton Mills, WV Engine Dynamometer Data

\begin{tabular}{|c|c|c|c|c|c|}
\hline \multicolumn{6}{|c|}{ ANN 1 Trained with Washington, PA2 1 Data } \\
\hline \multicolumn{3}{|c|}{ ANN 2 Trained with Manufacturer A Sabraton to Bruceton Data } \\
\hline \multirow{2}{*}{ Verification Data } & \multirow{2}{*}{ Run } & \multicolumn{2}{|c|}{ Predicted $(\mathrm{g} / \mathrm{bhp}$-hr) } & \multicolumn{2}{c|}{ Meas. Engine Dyno (g/bhp-hr) } \\
\cline { 2 - 6 } & & $\mathrm{CO}$ & $\mathrm{HC}$ & $\mathrm{CO}$ & $\mathrm{HC}$ \\
\hline \multirow{3}{*}{ Sab2Bruceton 1 } & 1 & 0.29 & 0.037 & 0.30 & 0.041 \\
\cline { 2 - 6 } & 2 & 0.45 & 0.019 & 0.30 & 0.041 \\
\cline { 2 - 6 } & 3 & 0.30 & 0.032 & 0.30 & 0.041 \\
\cline { 2 - 6 } & Average & 0.35 & 0.029 & 0.30 & 0.041 \\
\hline \multirow{3}{*}{ Bruceton2Sab 1 } & 1 & 0.29 & 0.037 & 0.30 & 0.041 \\
\cline { 2 - 6 } & 2 & 0.34 & 0.048 & 0.30 & 0.041 \\
\cline { 2 - 6 } & 3 & 0.27 & 0.027 & 0.30 & 0.041 \\
\cline { 2 - 6 } & Average & 0.30 & 0.040 & 0.30 & 0.041 \\
\hline
\end{tabular}

It was determined that when the ANN was trained with in-use data from the Washington, PA route and engine dynamometer data from the Bruceton Mills, WV cycle, and then used to predict emissions associated with Bruceton Mill, WV routes that a percent difference as low as 9.1\% was achieved for $\mathrm{HC}$. The average percent difference between measured and predicted $\mathrm{CO}$ emissions was determined to be $18.2 \%$ for the Sab2Bruceton 1 data set, and $9.1 \%$ for the Bruceton2Sab 1 data set. 
Table 6.6.2 Comparison of Predicted Values of CO and HC to Measured Values from Engine Dynamometer Data for ANN Trained with Washington, PA2 1 and Sabraton, WV to Bruceton Mills, WV Engine Dynamometer Data

\begin{tabular}{|c|c|c|c|}
\hline \multicolumn{4}{|c|}{ ANN 1 Trained with Wash PA2 1 Data } \\
\hline ANN 2 Trained with Manufacturer A Sabraton to Bruceton Data \\
\hline \multirow{2}{*}{ Verification Data } & Run & $\begin{array}{c}\text { \% Diff. Pred. } \\
\text { CO vs. Meas. }\end{array}$ & $\begin{array}{c}\text { \% Diff. Meas. } \\
\text { HC vs. Meas. }\end{array}$ \\
\hline \multirow{4}{*}{ Sab2Bruceton 1 } & 1 & 2.1 & 9.1 \\
\cline { 2 - 4 } & 2 & 51.9 & 53.3 \\
\cline { 2 - 4 } & 3 & 0.6 & 20.0 \\
\cline { 2 - 4 } & Average & 18.2 & 27.5 \\
\hline \multirow{3}{*}{ Bruceton2Sab 1 } & 1 & 1.9 & 8.6 \\
\cline { 2 - 4 } & 2 & 14.8 & 18.3 \\
\cline { 2 - 4 } & 3 & 10.6 & 32.6 \\
\cline { 2 - 4 } & Average & 9.1 & 19.8 \\
\hline
\end{tabular}

Tables 6.6.3 and 6.6.4 compare the predicted integrated brake-specific $\mathrm{HC}$ and $\mathrm{CO}$ emissions to the values that were measured during engine dynamometer testing employing the cycle that simulated the Bruceton Mills, WV route. The average predicted integrated emissions for $\mathrm{CO}$ and HC were $0.35 \mathrm{~g} / \mathrm{bhp}-\mathrm{hr}$ and $0.029 \mathrm{~g} / \mathrm{bhp}-\mathrm{hr}$, respectively. The measured emissions values associated with the engine dynamometer for $\mathrm{CO}$ and $\mathrm{HC}$ were $0.30 \mathrm{~g} / \mathrm{bhp}-\mathrm{hr}$ and $0.041 \mathrm{~g} / \mathrm{bhp}-\mathrm{hr}$, respectively.

Table 6.6.3 Predicted and Measured Values for HC and CO for ANN Trained with Bruceton2Sab 1 and Washington, PA1 Engine Dynamometer Data

\begin{tabular}{|l|c|c|c|c|c|}
\hline \multicolumn{6}{|c|}{ ANN 1 Trained with Bruceton2Sab 1 Data } \\
\hline \multicolumn{6}{|c|}{ ANN 2 Trained with Manufacturer A Washington, PA1 Data } \\
\hline \multirow{3}{*}{ Verification Data } & \multirow{2}{*}{ Run } & \multicolumn{2}{|c|}{ Predicted $(\mathrm{g} /$ bhp-hr) } & \multicolumn{2}{c|}{ Meas. Engine Dyno (g/bhp-hr) } \\
\cline { 2 - 6 } & & $\mathrm{CO}$ & $\mathrm{HC}$ & $\mathrm{CO}$ & $\mathrm{HC}$ \\
\hline \multirow{3}{*}{ Sab2Bruceton 1 } & 1 & 0.40 & 0.049 & 0.30 & 0.041 \\
\cline { 2 - 6 } & 2 & 0.43 & 0.045 & 0.30 & 0.041 \\
\cline { 2 - 6 } & 3 & 0.38 & 0.046 & 0.30 & 0.041 \\
\cline { 2 - 6 } & Average & 0.40 & 0.046 & 0.30 & 0.041 \\
\hline \multirow{3}{*}{ Sab2Bruceton 2 } & 1 & 0.36 & 0.023 & 0.30 & 0.041 \\
\cline { 2 - 6 } & 2 & 0.45 & 0.025 & 0.30 & 0.041 \\
\cline { 2 - 6 } & 3 & 0.38 & 0.047 & 0.30 & 0.041 \\
\cline { 2 - 6 } & Average & 0.40 & 0.030 & 0.30 & 0.041 \\
\hline
\end{tabular}


It was determined that when the ANN was trained with in-use data from the Bruceton Mills, WV route and engine dynamometer data from the Washington, PA cycle, and then used to predict emissions associated with Bruceton Mill, WV routes that a percent difference as low as $11.1 \%$ was achieved for HC. The average percent difference between measured and predicted $\mathrm{CO}$ emissions was determined to be $34.2 \%$ for the Sab2Bruceton 1 data set, and $33.2 \%$ for the Bruceton2Sab 1 data set.

Table 6.6.7 Comparison of Predicted Values of CO and HC to Measured Values from Engine Dynamometer Data for ANN Trained with Bruceton2Sab 1 and Washington, PA1 Engine Dynamometer Data

\begin{tabular}{|c|c|c|c|}
\hline \multicolumn{4}{|c|}{ ANN 1 Trained with Bruceton2Sab 1 Data } \\
\hline \multicolumn{3}{|c|}{ ANN 2 Trained with Manufacturer A PA1 Data } \\
\hline \multirow{2}{*}{ Verification Data } & Run & $\begin{array}{c}\text { \% Diff. Pred. } \\
\text { CO vs. Meas. }\end{array}$ & $\begin{array}{c}\text { \% Diff. Meas. } \\
\text { HC vs. Meas. }\end{array}$ \\
\hline \multirow{4}{*}{ Sab2Bruceton 1 } & 1 & 33.6 & 20.5 \\
\cline { 2 - 4 } & 2 & 43.6 & 11.1 \\
\cline { 2 - 4 } & 3 & 25.5 & 12.3 \\
\cline { 2 - 4 } & Average & 34.2 & 14.7 \\
\hline \multirow{4}{*}{ Sab2Bruceton 2 } & 1 & 21.8 & 44.4 \\
\cline { 2 - 4 } & 2 & 51.8 & 38.3 \\
\cline { 2 - 4 } & 3 & 26.0 & 15.6 \\
\cline { 2 - 4 } & Average & 33.2 & 32.8 \\
\hline
\end{tabular}

\subsection{Engine Emissions Predictions}

In order to demonstrate that the model was applicable to a range of engine model years, the ANN was used to predict emissions associated with a 2002 engines from engine Manufacturer B and engine Manufacturer C. Engine dynamometer testing data was not available for these engines, so in-use data was employed to train both the vehicle and the emissions modules of the ANN. Table 6.7.1 displays the verification tests conducted for the Manufacturer B engine. It was shown that when the model was trained with in-use data from the Washington, PA 2 route, it was able to predict the emissions associated with the Washington, PA 1 route within 17\% of the measured values. When the model was trained with data from the Washington, PA 1 route it performed best when predicting the emissions associated with the travel from the end of the 
Washington, PA 3 route to Sabraton, WV. The ANN was able to predict the emissions associated with the travel from the end of Washington, PA 3 to Sabraton, WV with an average percent difference of $3.2 \%$ for $\mathrm{CO}_{2}$ and $12.0 \%$ for $\mathrm{NOx}$, when compared to measured values. The ANN was then trained with in-use data acquired from traveling from the end of the Washington, PA 3 route to Sabraton, WV, and was used to predict the emissions associated with the route from Sabraton, WV to Washington, PA. It was determined that this training, and verification combination resulted in an average percent difference between the predicted and measured emissions values of $0.7 \%$ for $\mathrm{NOx}$ and $1.9 \%$ for $\mathrm{CO}_{2}$.

Table 6.7.1 Comparison of Predicted and Measured Values of $\mathrm{NOx}$ and $\mathrm{CO}_{2}$ for the $\mathrm{ANN}$ trained with In-Use Data

\begin{tabular}{|c|c|c|c|}
\hline \multicolumn{4}{|c|}{ ANN Trained with Washington PA2 Data } \\
\hline Verification Data & Run & $\begin{array}{c}\text { \% Diff. } \\
\text { NOx }\end{array}$ & $\begin{array}{c}\text { \% Diff. } \\
\mathrm{CO}_{2}\end{array}$ \\
\hline \multirow{4}{*}{ Washington, PA1 } & 1 & 13.0 & 20.0 \\
\hline & 2 & 14.6 & 16.1 \\
\hline & 3 & 16.4 & 15.0 \\
\hline & Average & 14.7 & 17.0 \\
\hline \multirow{4}{*}{ Sabraton, WV to Washington, $P A$} & 1 & 17.6 & 4.8 \\
\hline & 2 & 24.7 & 11.4 \\
\hline & 3 & 24.4 & 1.4 \\
\hline & Average & 22.2 & 5.9 \\
\hline \multirow{4}{*}{ Washington, PA3 to Sabraton, WV } & 1 & 12.2 & 1.4 \\
\hline & 2 & 7.6 & 1.1 \\
\hline & 3 & 16.3 & 7.1 \\
\hline & Average & 12.0 & 3.2 \\
\hline \multicolumn{4}{|c|}{ ANN Trained with Washington, PA3 to Sabraton, WV Data } \\
\hline Verification Data & Run & $\begin{array}{c}\text { \% Diff. } \\
\text { NOx }\end{array}$ & $\begin{array}{c}\text { \% Diff. } \\
\mathrm{CO}_{2}\end{array}$ \\
\hline \multirow{4}{*}{ Sabraton, WV to Washington, $P A$} & 1 & 0.3 & 1.8 \\
\hline & 2 & 0.8 & 1.1 \\
\hline & 3 & 0.9 & 2.6 \\
\hline & Average & 0.7 & 1.9 \\
\hline
\end{tabular}

*The highlighted values are shown in the following figures

Figure 6.7.1 shows the comparison of the engine speed predicted for the Washington, PA 1 route to the actual measured engine speed recorded during in-use testing. For this prediction, the ANN 
was trained with data obtained during in-use testing of the Washington, PA 2 route. The comparison between predicted and measured engine torque is shown in Figure 6.7.2. The data shown in the following figures corresponds to the highlighted run in the above table.

The ANN did not experience the under-prediction issues with engine speed during idle conditions, as it had in the previous results discussed, however the minimum predicted engine torque was still below the actual torque of the route. The minimum torque in the training data was determined to be $-32 \mathrm{ft}-\mathrm{lbs}$, while a minimum torque of $-12,750 \mathrm{ft}-\mathrm{lbs}$ occurred in the verification data. The torque of $-12,750 \mathrm{ft}-1 \mathrm{bs}$ was determined to be the result of an error in postprocessing the data, and not a realistic torque experienced by the engine. The data file was examined and it was determined at the time of the minimum torque occurrence the torque and speed reported by the ECU were not orders of magnitudes different from those experienced in the prior to or after the measurement being examined. It was determined that a post-processing error was responsible for the torque of $-12,750 \mathrm{ft}-1 \mathrm{bs}$ because it was a calculated torque value and not one recorded by the data acquisition system.

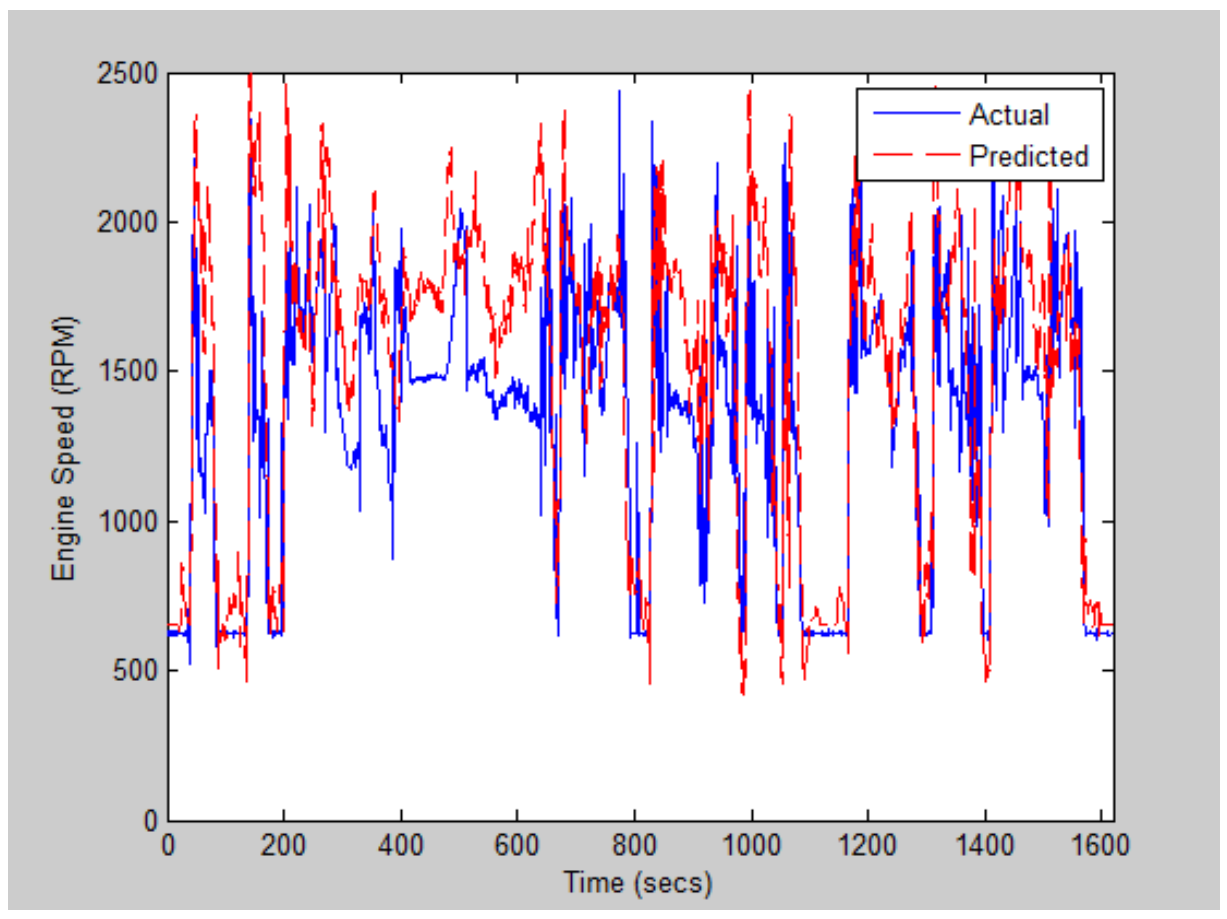

Figure 6.7.1: Predicted and Measured Engine Speed for Washington, PA 1 When the ANN Was Trained with Data from Washington, PA 2 


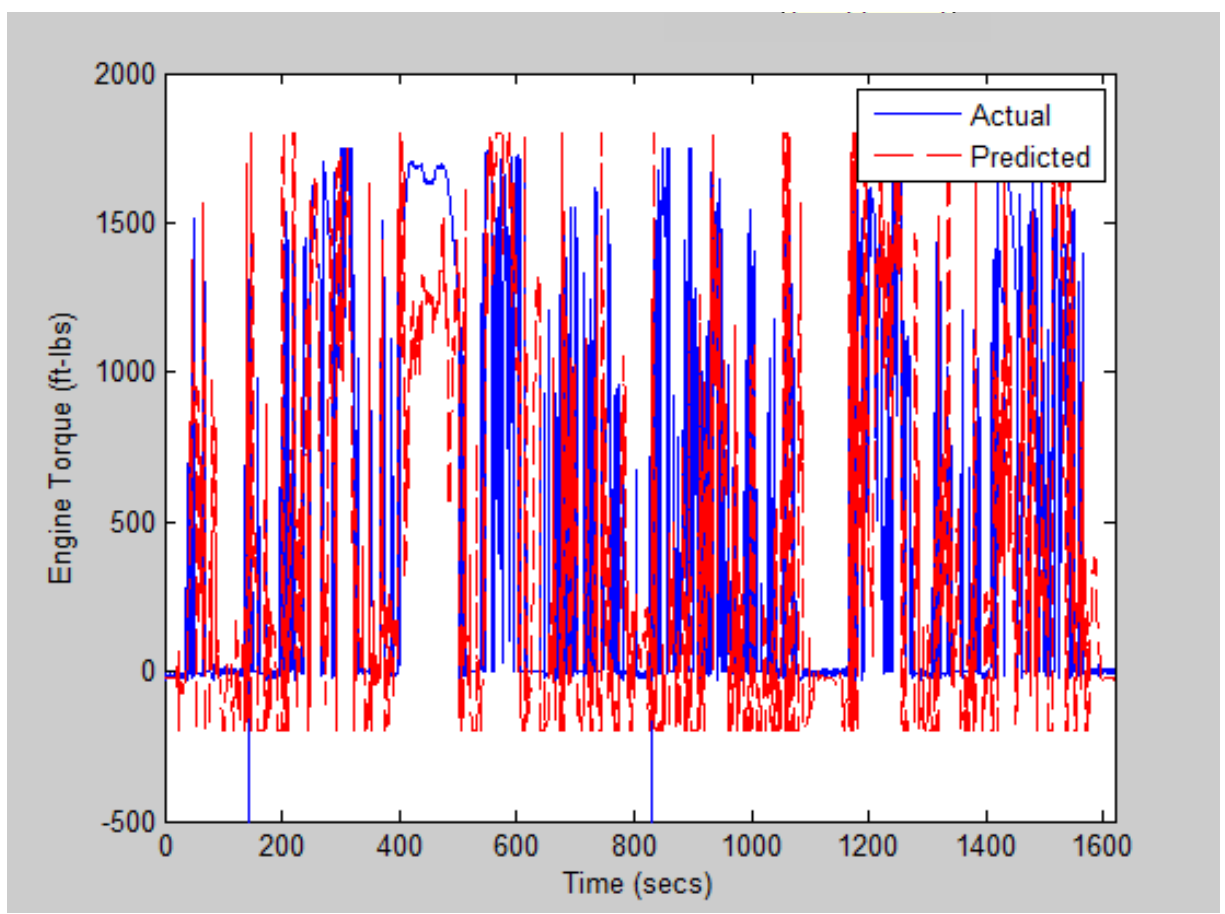

Figure 6.7.2: Predicted and Measured Torque for Washington, PA 1 When the ANN Was Trained with Data from Washington, PA 2

Figures 6.7.3 and 6.7.4 compare the measured and predicted values for the emissions associated with the Washington, PA 1 route. It was determined that the average percent difference between the predicted and measured values was $14.7 \%$ for $\mathrm{NOx}$, and $17.0 \%$ for $\mathrm{CO}_{2}$. 


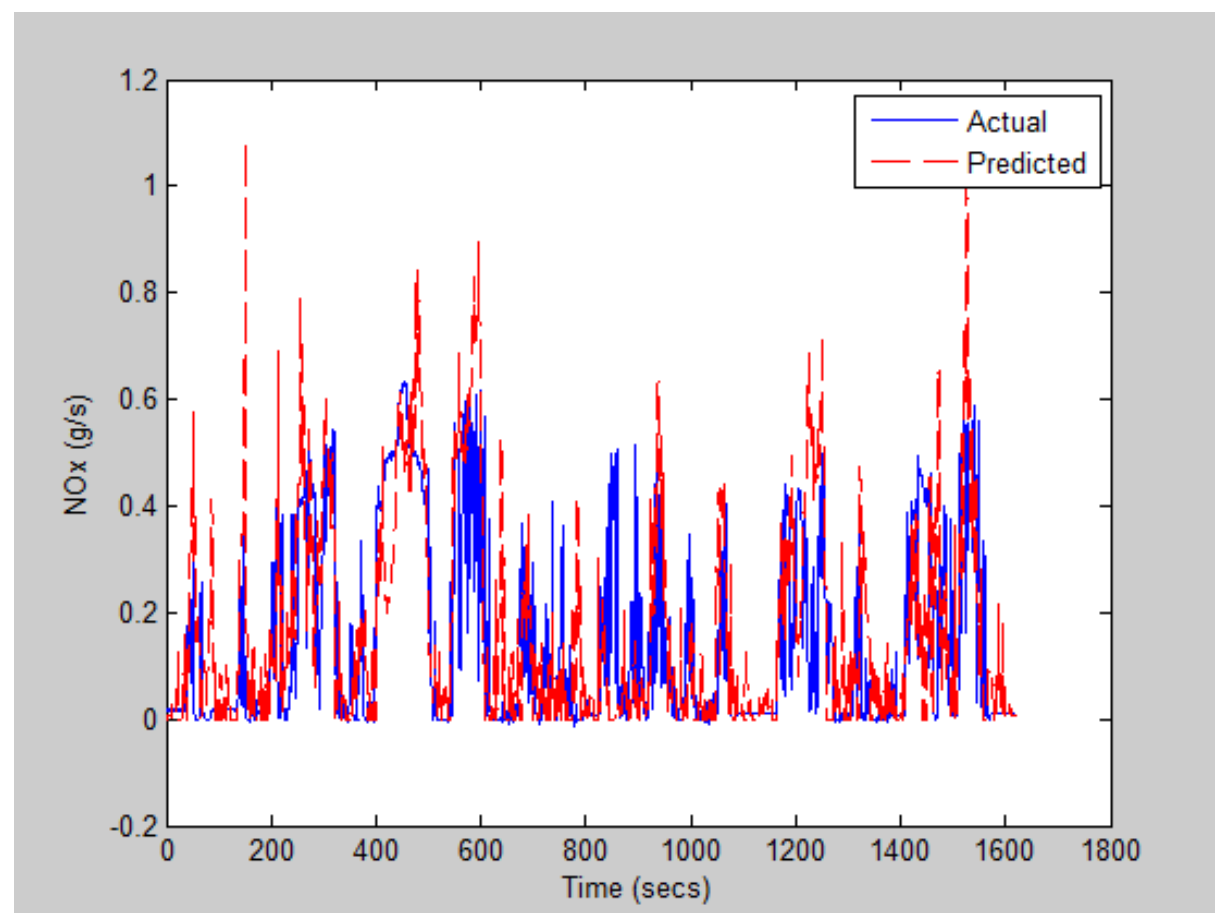

Figure 6.7.3: Predicted and Measured NOx Emissions for Washington, PA 1 When the ANN Was Trained with Data from Washington, PA 2

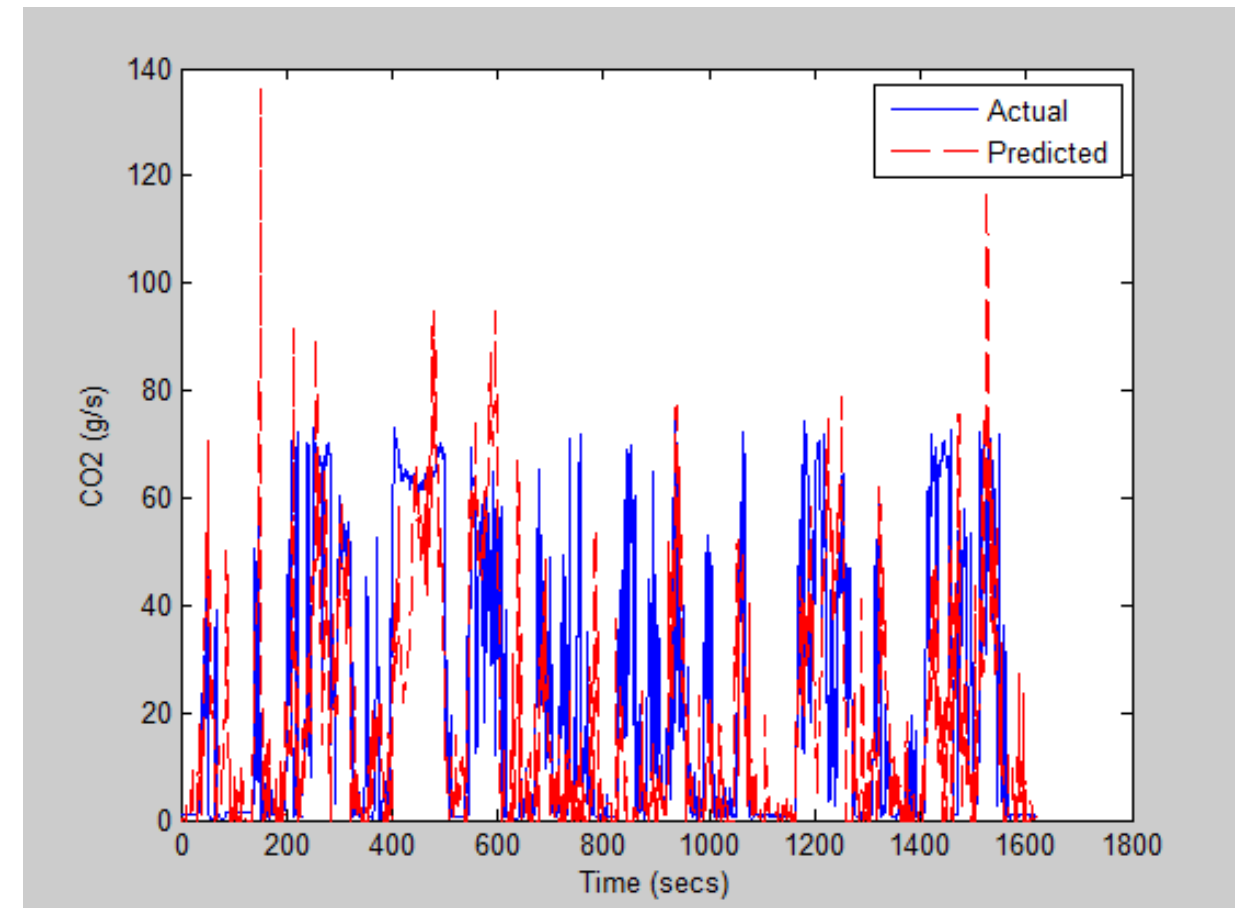

Figure 6.7.4: Predicted and Measured $\mathrm{CO}_{2}$ Emissions for Washington, PA 1 When the ANN Was Trained with Data from Washington, PA 2 
The ANN was also trained with data obtained from in-use testing which occurred on the route between the Washington, PA 3 route and Sabraton, WV. When the ANN was trained with this data, and used to predict emissions associated with a vehicle traveling from Sabraton, WV to Washington, PA the percent differences between the measured and predicted integrated emissions were determined to be as low as $0.3 \%$ for NOx. Figures 6.7 .5 and 6.7.6 show the differences between the measured and the predicted engine speed and torque. It is shown that that the ANN failed to accurately predict the engine speed when instantaneous spikes were present, such as the one that occurred at 2581 seconds. The data was examined and determined that this spike in the figure was the result of the engine speed changing from $1460 \mathrm{rpm}$ to 2070 rpm in 0.4 seconds. An equally significant change in torque and power were not present at 2581 seconds. Since the engine speed data preceding and proceeding the data at 2581 seconds were not shown to vary as greatly as the data at 2581 seconds, this was determined to be an error in the data and not representative of the actual engine speed experienced during the route.

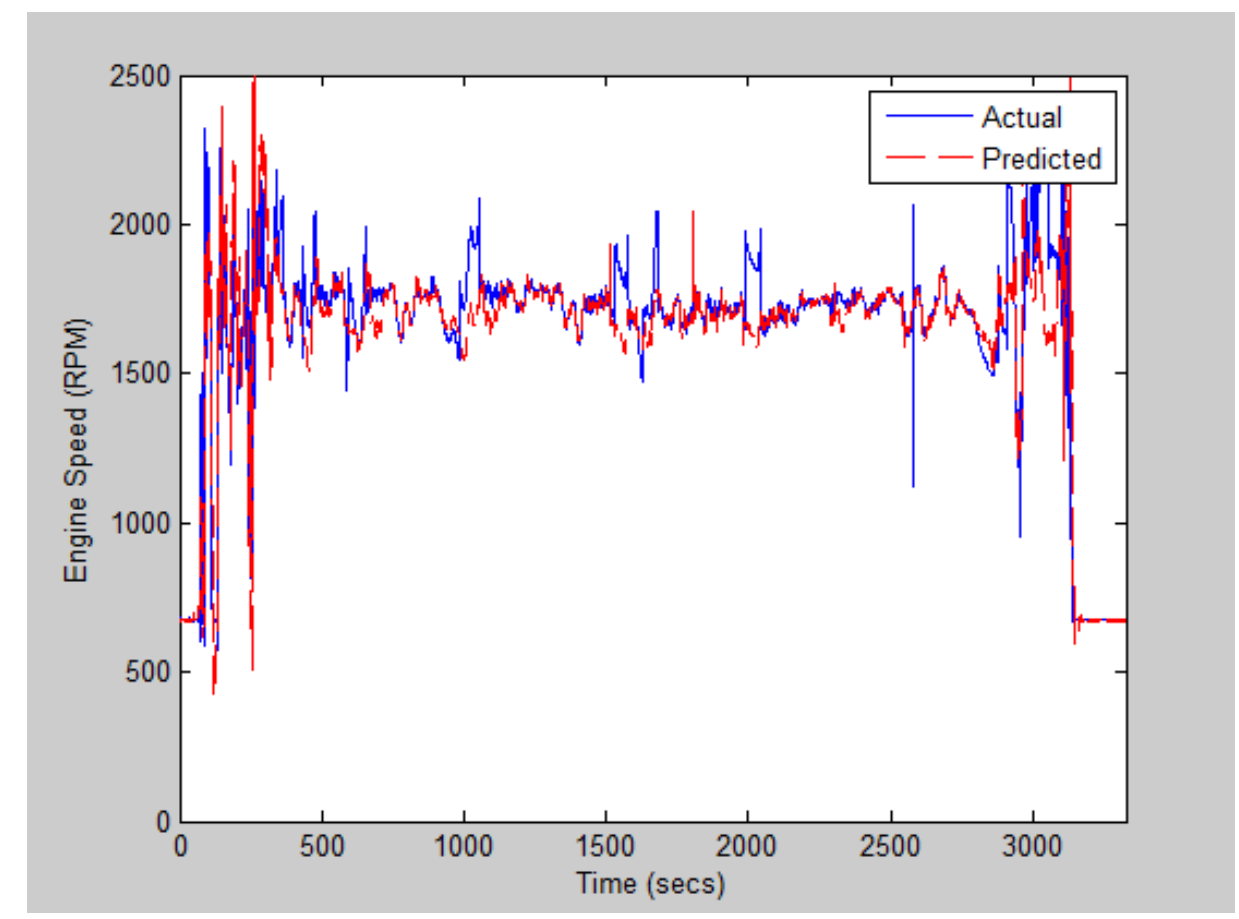

Figure 6.7.5: Predicted and Measured Engine Speed for Sabraton, WV to Washington, PA When the ANN Was Trained with Data from Washington, PA 3 to Sabraton, WV Route 


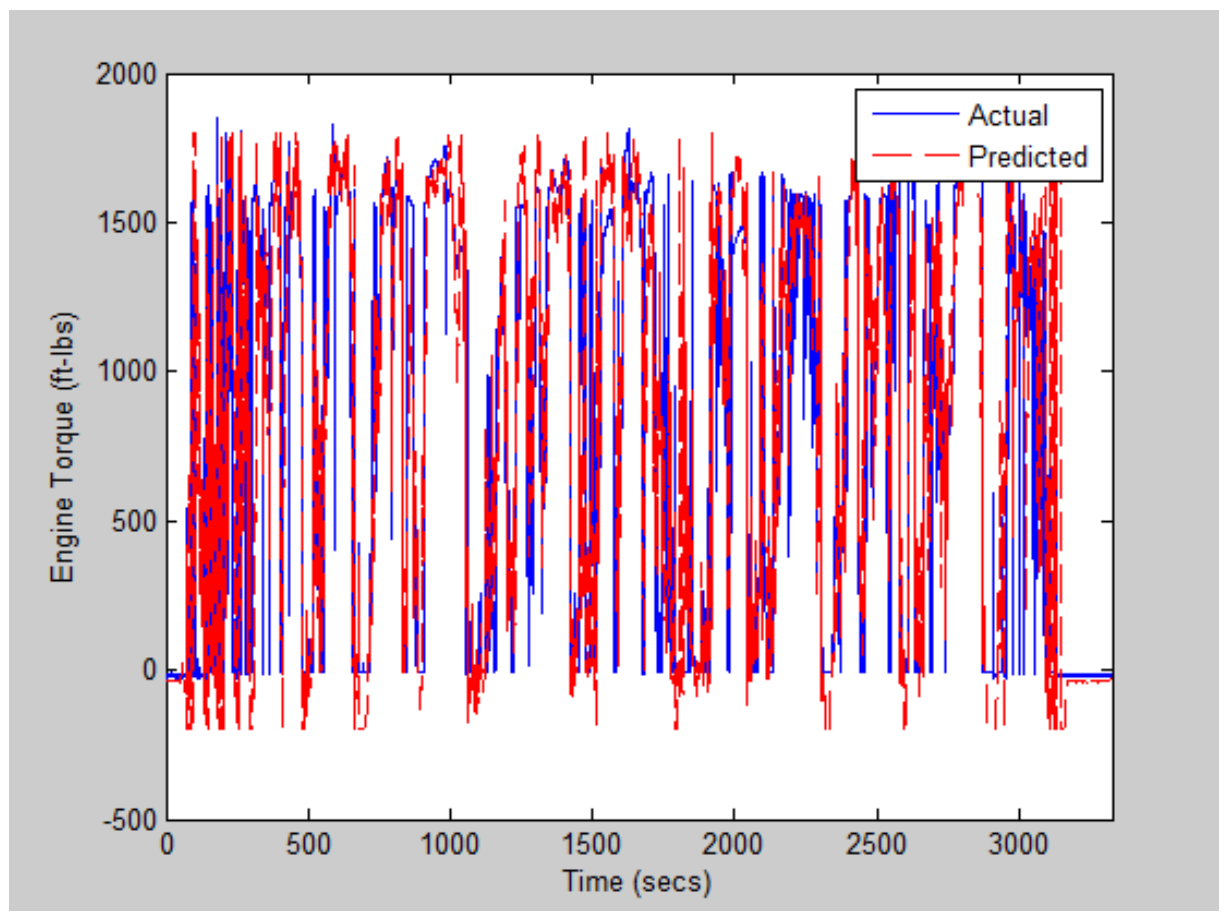

Figure 6.7.6: Predicted and Measured Torque for Sabraton, WV to Washington, PA When the ANN Was Trained with Data from Washington, PA 3 to Sabraton, WV Route

Figures 6.7.7 and 6.7.8 compare the measured and predicted values for the emissions associated with a vehicle traveling form Sabraton, WV to Washington, PA. It was determined that the average percent difference between the predicted and measured values was $0.7 \%$ for NOx, and $1.9 \%$ for $\mathrm{CO}_{2}$. It was evident that the ANN was able to predict emissions with less than $2 \%$ difference from the measured values, when the predicted engine speed did not account for instantaneous magnitude increases in the actual data.

The agreement between the measured and predicted emissions values was better when both modules of the ANN were trained with in-use data. This is attributed to the fact that the same measurement equipment was employed in both sets if data, the one used to train the emissions module, and the one used to train vehicle module of the ANN. Also since laboratory data was not used, the dispersion and measurement delays due to the dilution tunnel were avoided. 


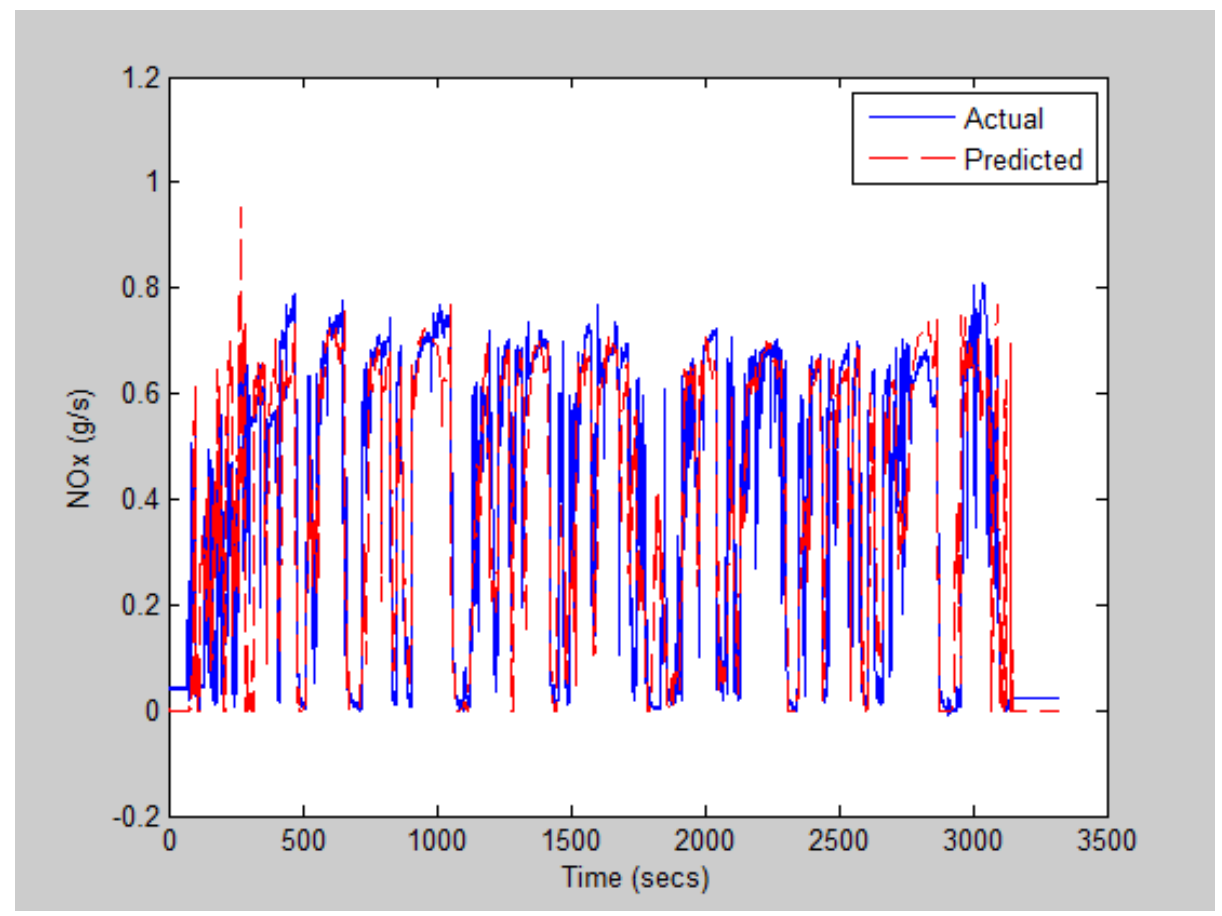

Figure 6.7.7: Predicted and Measured NOx Emissions for Sabraton, WV to Washington, PA When the ANN Was Trained with Data from Washington, PA 3 to Sabraton, WV Route

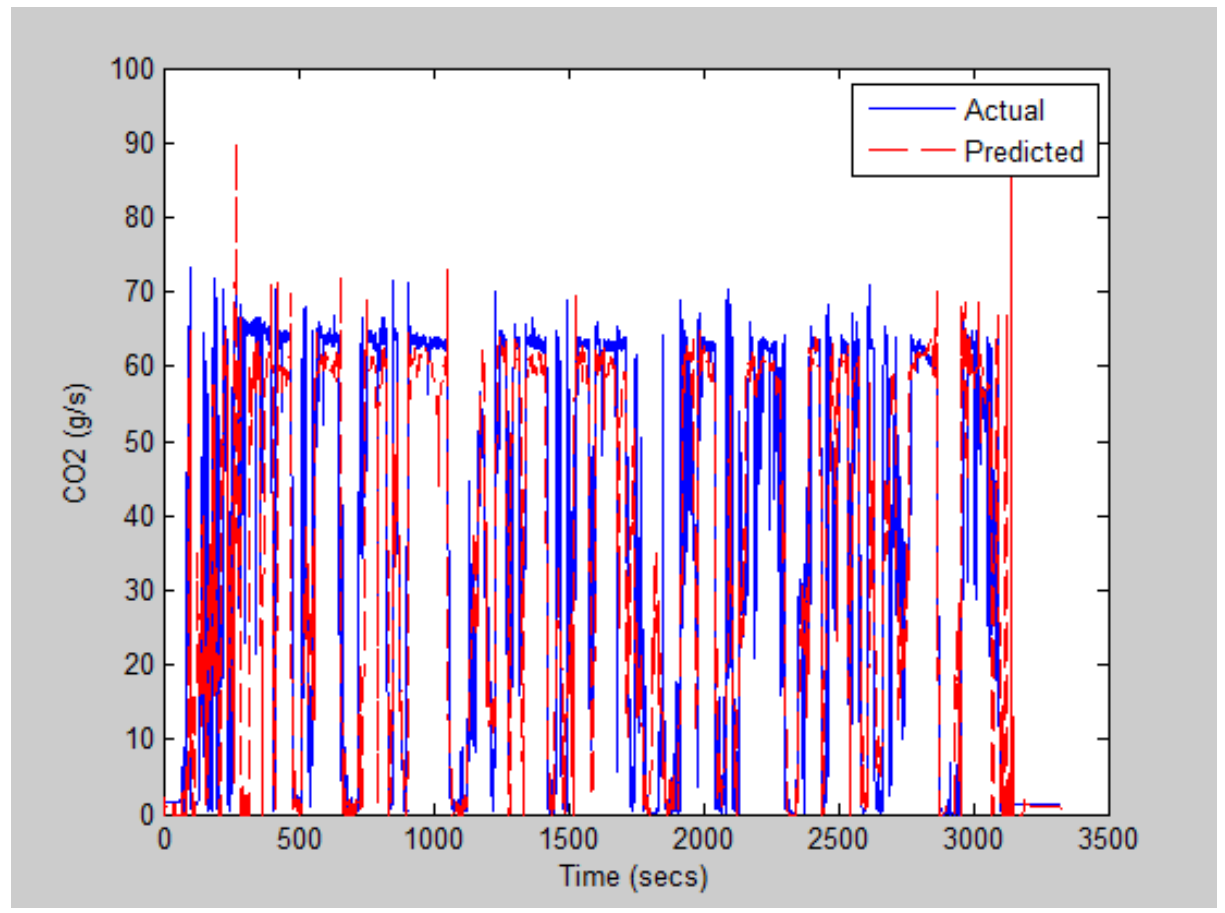

Figure 6.7.8: Predicted and Measured $\mathrm{CO}_{2}$ Emissions for Sabraton, WV to Washington, PA When the ANN Was Trained with Data from Washington, PA 3 to Sabraton, WV Route 
The ANN was also used to predict emissions associated with a 2002 engine from Manufacturer C. Engine dynamometer testing data was not available for this engine, so in-use data was employed to train both the vehicle and the emissions modules of the ANN. It was shown that when the model was trained with in-use data from the route of Washington, PA 3 to Sabraton, $\mathrm{WV}$, it was able to predict the emissions associated with route from Sabraton, WV to Washington, PA within $4 \%$ of the measured values. The ANN was also trained with in-used data from Sabraton, WV to Washington, PA, and was used to predict emissions associated with the route from Washington, PA 3 to Sabraton, PA. The ANN was able to predict the emissions associated with the travel from the end of Washington, PA 3 to Sabraton, WV with an average percent difference of $4.4 \%$ for $\mathrm{CO}_{2}$ and $13.5 \%$ for $\mathrm{NOx}$, when compared to measured values.

Table 6.7.2 Comparison of Predicted and Measured Values of $\mathrm{NOx}$ and $\mathrm{CO}_{2}$ for the $\mathrm{ANN}$ trained with In-use Data

\begin{tabular}{|c|c|c|c|}
\hline \multicolumn{4}{|c|}{ ANN Trained with Washington, PA 3 to Sabraton, WV Data } \\
\hline Verification Data & Run & $\begin{array}{c}\text { \% Diff. } \\
\text { NOx }\end{array}$ & $\begin{array}{c}\text { \% Diff. } \\
\mathrm{CO}_{2}\end{array}$ \\
\hline \multirow{4}{*}{ Sabraton, WV to Washington, $P A$} & 1 & 2.2 & 0.7 \\
\hline & 2 & 3.6 & 2.0 \\
\hline & 3 & 1.0 & 0.1 \\
\hline & Average & 2.3 & 0.9 \\
\hline \multicolumn{4}{|c|}{ ANN Trained with Sabraton, WV to Washington, PA Data } \\
\hline Verification Data & Run & $\begin{array}{c}\text { \% Diff. } \\
\text { NOx }\end{array}$ & $\begin{array}{c}\text { \% Diff. } \\
\mathrm{CO}_{2}\end{array}$ \\
\hline \multirow{4}{*}{ Washington, PA to Sabraton, WV } & 1 & 10.4 & 1.4 \\
\hline & 2 & 15.2 & 5.6 \\
\hline & 3 & 14.8 & 6.4 \\
\hline & Average & 13.5 & 4.4 \\
\hline
\end{tabular}

*The highlighted values are shown in the following figures

Figures 6.7.9 and 6.7.10 compare the predicted and actual measured values of $\mathrm{NOx}$ and $\mathrm{CO}_{2}$ associated with the route from Sabraton, WV to Washington, PA. The percent difference between measured and predicted $\mathrm{NOx}$ and $\mathrm{CO}_{2}$ emissions for the third verification run were $1.0 \%$ and $0.1 \%$, respectively. 


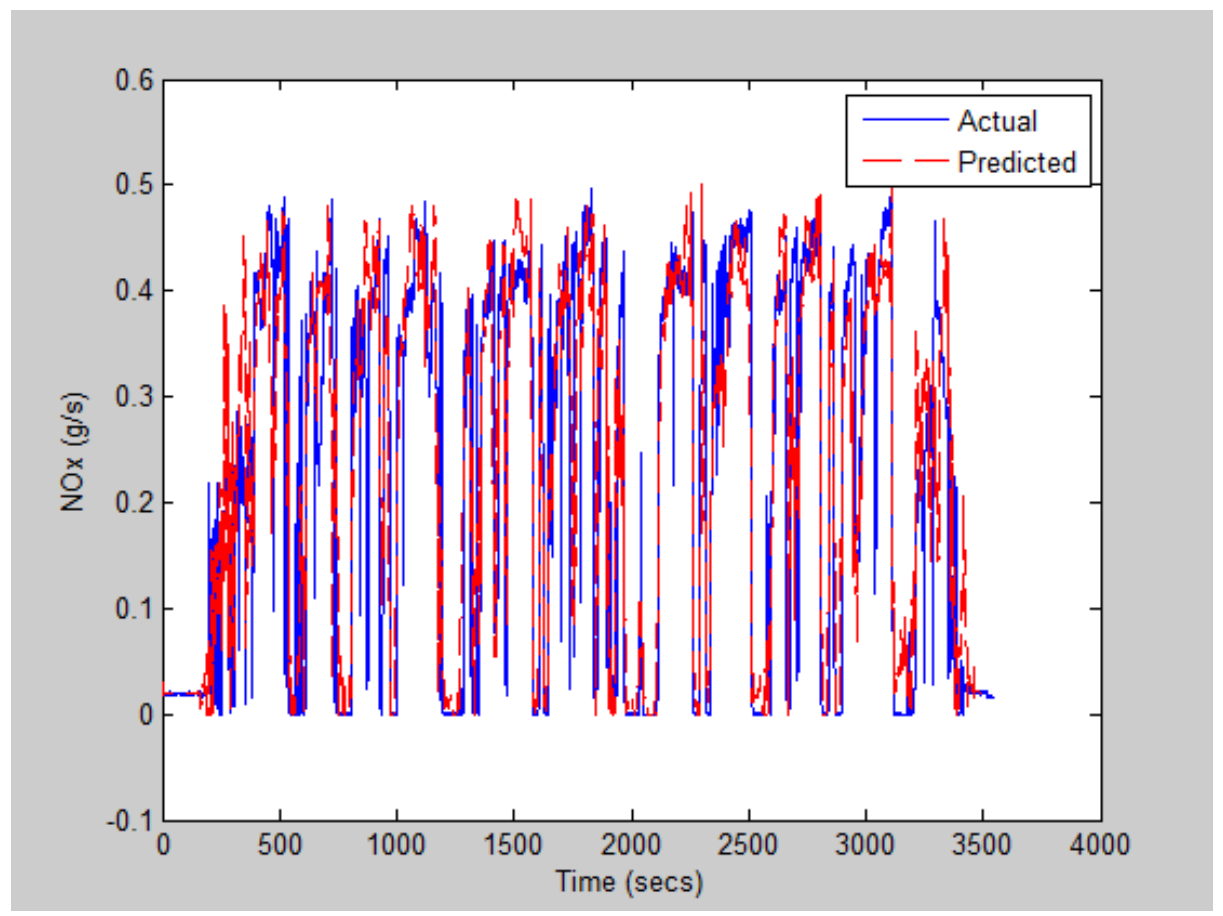

Figure 6.7.9: Predicted and Measured NOx Emissions for Sabraton, WV to Washington, PA When the ANN Was Trained with Data from Washington, PA 3 to Sabraton, WV Route

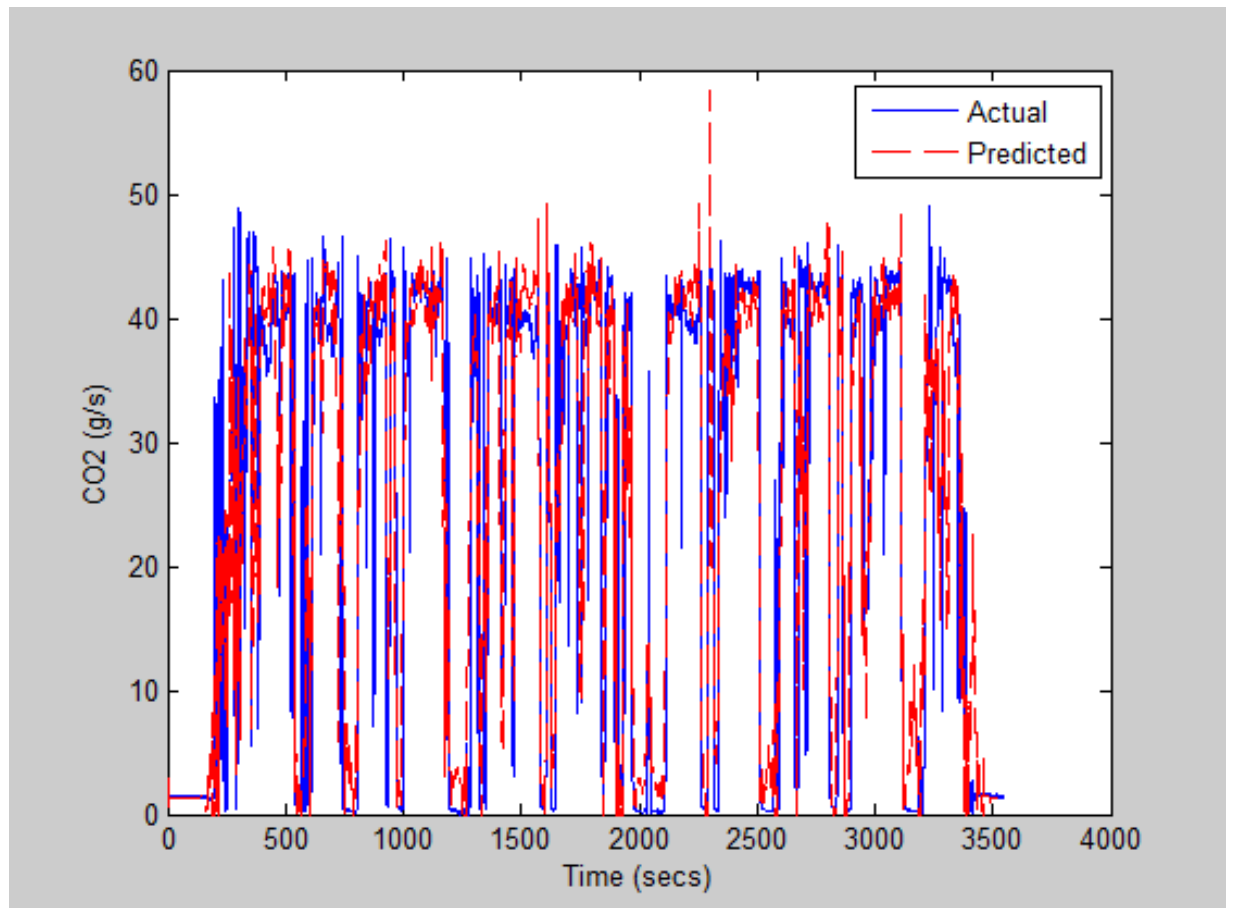

Figure 6.7.10: Predicted and Measured $\mathrm{CO}_{2}$ Emissions for Sabraton, WV to Washington, PA When the ANN Was Trained with Data from Washington, PA 3 to Sabraton, WV Route 
The ANN was also trained with in-used data from Sabraton, WV to Washington, PA, and was used to predict emissions associated with the route from Washington, PA 3 to Sabraton, WV. Figures 6.7.11 and 6.7.12 display a comparison between predicted and measured values of NOx and $\mathrm{CO}_{2}$ for the route from Washington, PA to Sabraton, WV. The average integrated percent difference between measured values for $\mathrm{NOx}$ and $\mathrm{CO}_{2}$ were determined to be $13.5 \%$ and $4.4 \%$, respectively.

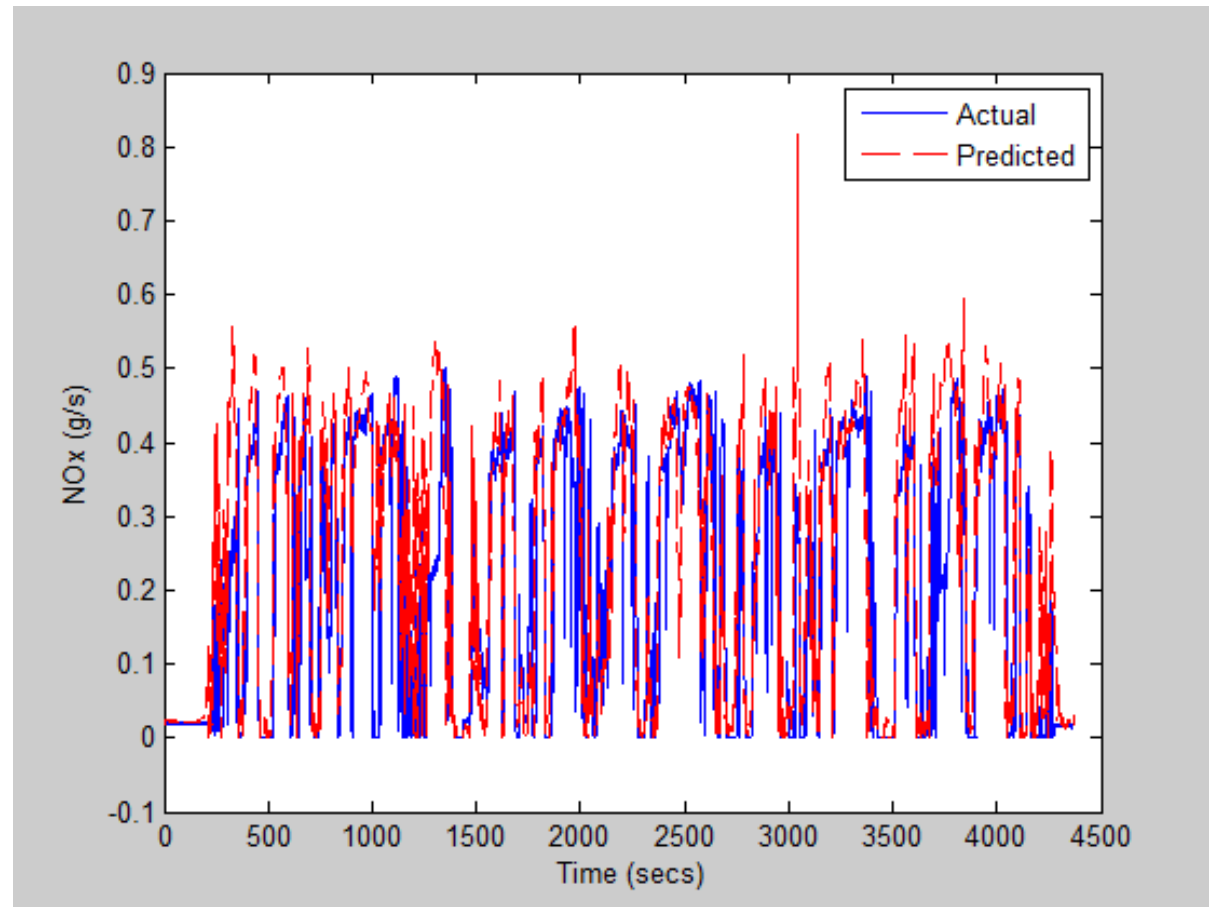

Figure 6.7.11: Predicted and Measured NOx Emissions for Washington, PA to Sabraton, WV When the ANN Was Trained with Data from Sabraton, WV to Washington, PA Route 


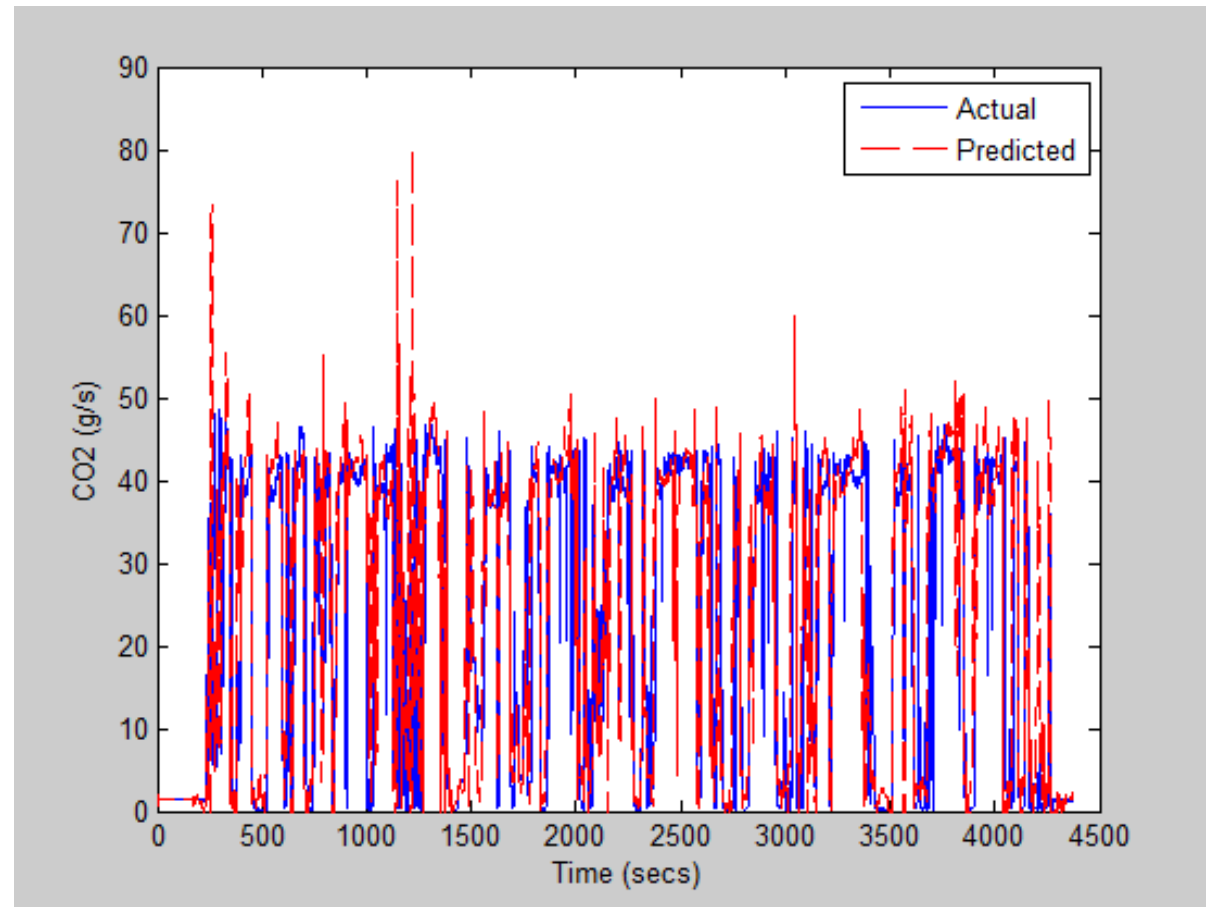

Figure 6.7.12: Predicted and Measured $\mathrm{CO}_{2}$ Emissions for Washington, PA to Sabraton, WV When the ANN Was Trained with Data from Sabraton, WV to Washington, PA Route

\subsection{Different Engines for Training and Verification}

A study was also conducted to determine with what accuracy the ANN could predict the emissions associated with a specific engine, when the ANN was trained with data from a different engine. For this case the ANN was trained with both in-use and engine dynamometer data from a $400 \mathrm{hp}$ engine of Manufacturer A, and then the ANN was used to predict the emissions associated with a $350 \mathrm{hp}$ engine produced by Manufacturer A. These two engines were similar models, with differing horsepower ratings.

The normalization ranges of engine speed and torque employed by the ANN were altered for the prediction of the different engine. The normalization bounds that were applied to the ANN were scaled by a ratio of maximum torque and the engine speed associated with the maximum torque between the training engine and the verification engine. The maximum torque for the $400 \mathrm{hp}$ engine was determined to be $1572 \mathrm{ft}-\mathrm{lbs}$, with a corresponding engine speed of $1258 \mathrm{rpm}$, while the maximum torque for the $350 \mathrm{hp}$ engine was $1350 \mathrm{ft}-\mathrm{lbs}$, with a corresponding engine speed of $1230 \mathrm{rpm}$. Prior to applying the ANN model to the verification data, the normalization ranges 
were scaled with the ratios of the torques and speeds discussed above. The data displayed in Table 6.8.1 was obtained when the ANN was trained with in-use data from the Washington, PA 2 route, and engine dynamometer data from the cycle developed to simulate the Bruceton Mills, WV route. The trained ANN was then used to predict the emissions associated with the Bruceton Mills, WV route and the Washington, PA 2 route. It was determined that the percent difference between the measured and predicted integrated emissions values associated with the Bruceton Mills, WV route for NOx was on average $17.6 \%$, while the percent difference for $\mathrm{CO}_{2}$ was $4.4 \%$. Figure 6.8.1 displays the comparison between predicted and measured NOx values for the first run using the Bruceton Data for verification. A comparison of the predicted and measured $\mathrm{CO}_{2}$ values is shown in Figure 6.8.2. A discrepancy between the predicted and measured emissions occurred between 900 and 1100 seconds. These prediction errors occurred in all of the verification runs that were conducted; the training and verification data were examined to determine the cause of this discrepancy. It was determined that the maximum engine speed for the $400 \mathrm{hp}$ engine was $2110 \mathrm{rpm}$, while the maximum engine speed for the 350 $\mathrm{hp}$ engine was $2140 \mathrm{rpm}$. The $400 \mathrm{hp}$ engine had a maximum torque of $1460 \mathrm{ft}-\mathrm{lbs}$, while the maximum torque of the $350 \mathrm{hp}$ engine was $1350 \mathrm{ft}-1 \mathrm{bs}$. The torques and speeds associated with the region being examined did not exceed the range of the training data, and did not represent any irregularities such as missing data points, or unexpected spikes in values. It was determined that the error prediction in this region was not due to data issues, and was due to the ability of the ANN to learn and predict the emissions associated with the specific conditions occurring during that time period. 
Table 6.8.1: Comparison of Measured and Predicted Emissions When the ANN was Trained with a $400 \mathrm{hp}$ Engine and Used To Predict a $350 \mathrm{hp}$ Engine

\begin{tabular}{|c|c|c|c|}
\hline \multicolumn{4}{|c|}{ ANN 1 Trained with Washington, PA2 1 Data } \\
\hline \multicolumn{3}{|c|}{ ANN 2 Trained with Manufacturer A Bruceton Data } \\
\hline Verification Data & Run & \% Diff. NOx & \% Diff. CO \\
\hline \multirow{3}{*}{$\begin{array}{c}\text { Sabraton, WV to } \\
\text { Bruceton Mills, WV }\end{array}$} & 1 & 11.4 & 2.7 \\
\cline { 2 - 4 } & 2 & 25.8 & 8.9 \\
\cline { 2 - 4 } & 3 & 15.6 & 1.8 \\
\hline \multirow{4}{*}{ Washington PA2 } & Average & 17.6 & 4.4 \\
\cline { 2 - 4 } & 1 & 10.1 & 3.5 \\
\cline { 2 - 4 } & 2 & 5.9 & 12.0 \\
\cline { 2 - 4 } & Average & 11.1 & 3.2 \\
\hline
\end{tabular}

*The highlighted values are shown in the following figures

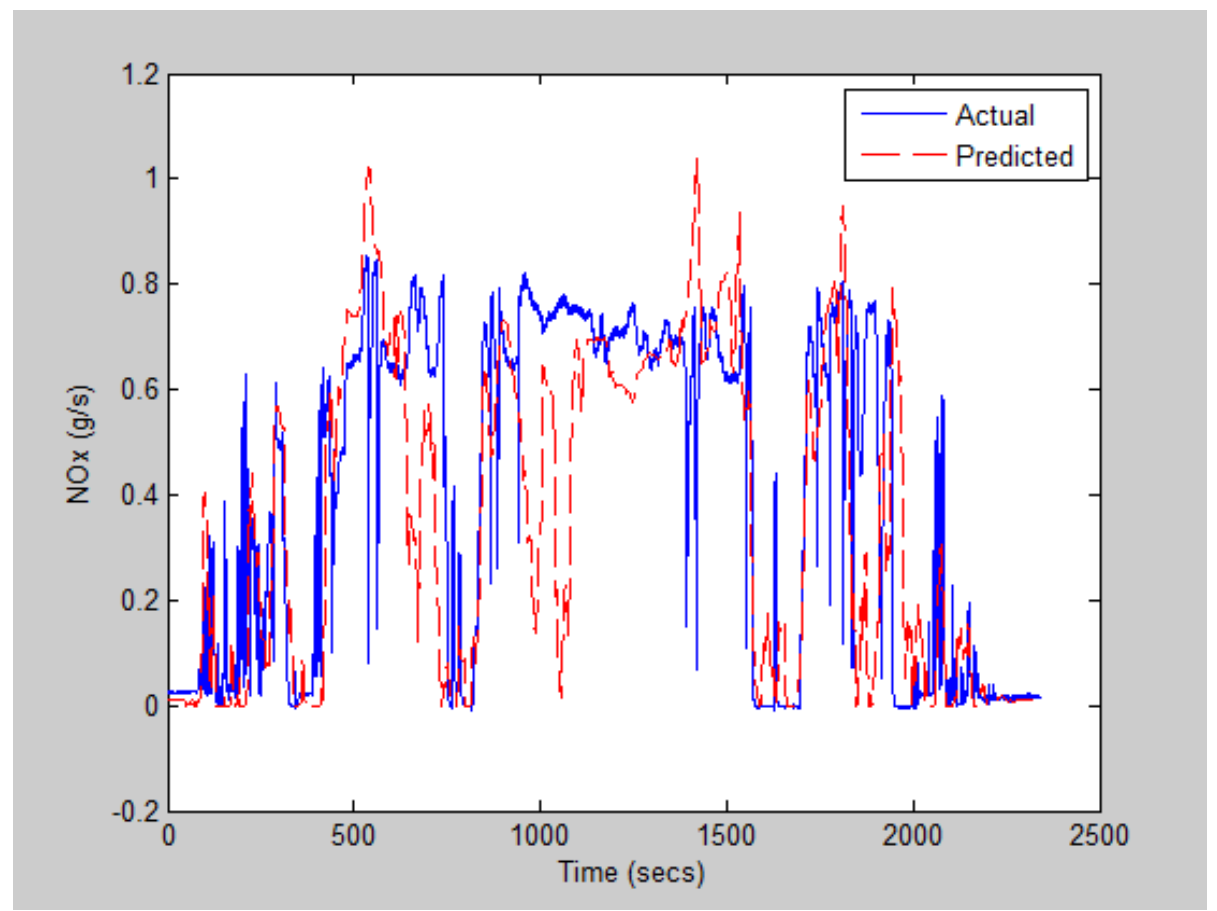

Figure 6.8.1: Predicted and Measured NOx Emissions for Bruceton Mills, WV route When the ANN Was Trained with Data from Washington, PA 2 Route 


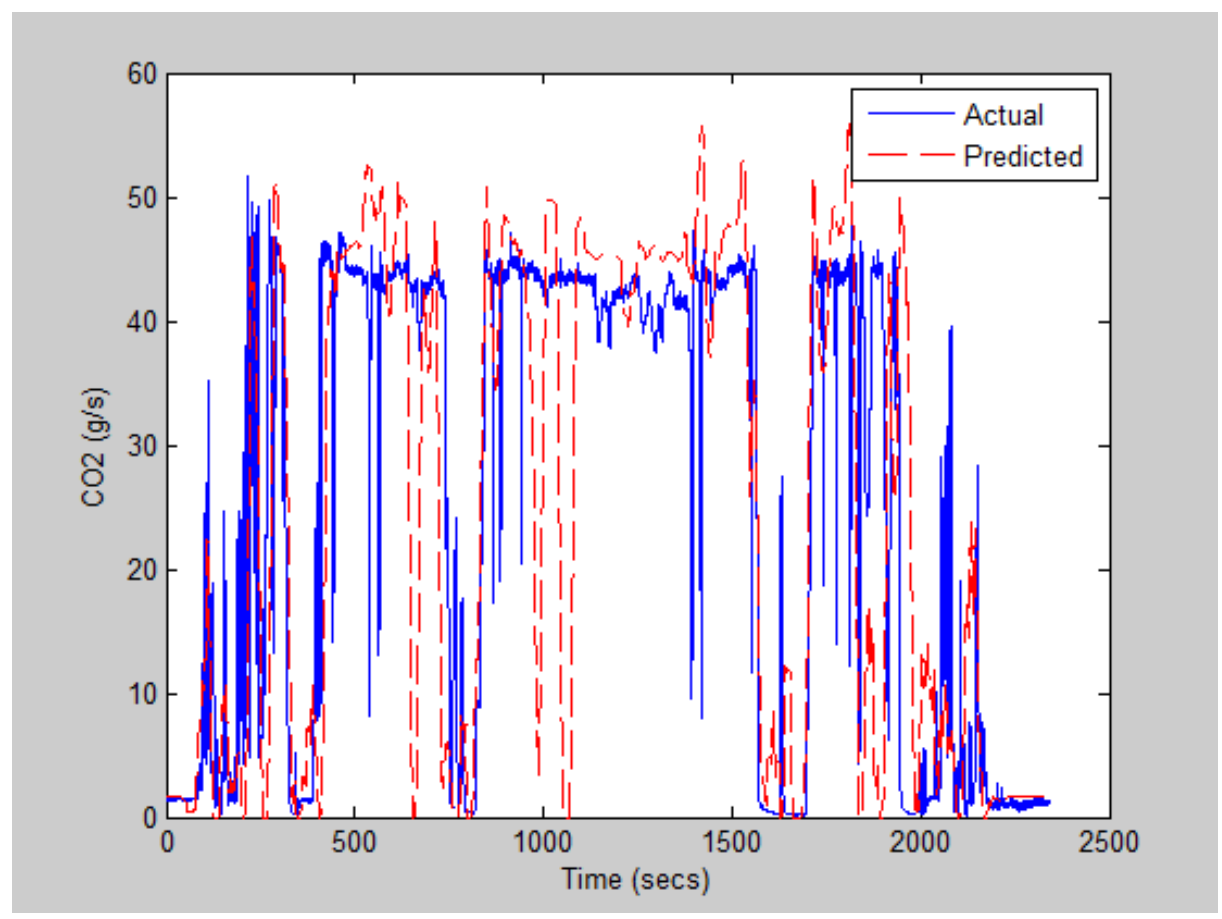

Figure 6.8.2: Predicted and Measured $\mathrm{CO}_{2}$ Emissions for Bruceton Mills, WV route When the ANN Was Trained with Data from Washington, PA 2 Route

The prediction of emissions associated with the $350 \mathrm{hp}$ engine also was performed with the ANN when it was trained with in-use data from the Washington, PA 2 route, and engine dynamometer from the cycle that simulated the Washington, PA route. The data discussed previously was obtained when the emissions module of the ANN was trained with data from the Bruceton Mills, WV engine dynamometer cycle. Table 6.8.2 displays the comparison of the measured and predicted integrated emissions for a Bruceton Mills, WV route and the Washington, PA 2 route. It was determined that the average percent difference between the measured and predicted NOx emissions was $24.4 \%$ for the Bruceton Mills, WV route, and $14.5 \%$ for the Washington, PA 2 route. The average percent difference between the measured and predicted $\mathrm{CO}_{2}$ emissions for the Bruceton Mills, WV route was 2.0\%, and 6.2\% for the Washington, PA 2 route. 
Table 6.8.2: Comparison of Measured and Predicted Emissions When the ANN was Trained with a $400 \mathrm{hp}$ Engine and Used To Predict a $350 \mathrm{hp}$ Engine

\begin{tabular}{|c|c|c|c|}
\hline \multicolumn{4}{|c|}{ ANN 1 Trained with Washington, PA2 1 Data } \\
\hline \multicolumn{4}{|c|}{ ANN 2 Trained with Manufacturer A Washington, PA1 Data } \\
\hline Verification Data & Run & \% Diff. NOx & $\%$ Diff. CO 2 \\
\hline \multirow{3}{*}{$\begin{array}{c}\text { Sabraton, WV to } \\
\text { Bruceton Mills, WV }\end{array}$} & 1 & 20.7 & 3.9 \\
\cline { 2 - 4 } & 2 & 25.8 & 0.02 \\
\cline { 2 - 4 } & 3 & 26.6 & 2.0 \\
\cline { 2 - 4 } & Average & 24.4 & 2.0 \\
\cline { 2 - 4 } & 1 & 11.4 & 8.0 \\
\cline { 2 - 4 } & 2 & 17.0 & 8.8 \\
\cline { 2 - 4 } Washington PA2 & 3 & 15.1 & 1.8 \\
\cline { 2 - 4 } & Average & 14.5 & 6.2 \\
\hline
\end{tabular}

*The highlighted values are shown in the following figures

Figures 6.8.3 and 6.8.4 display a comparison of the predicted and measured values for NOx and $\mathrm{CO}_{2}$ emissions. The data in the figures was corresponds to the first run of the ANN predicting the emissions associated with the Washington, PA 2 route, indicated by the highlighted row in the above table. The ANN was determined to over-predict both $\mathrm{NOx}$ and $\mathrm{CO}_{2}$ emissions for the Washington, PA 2 cycle. 


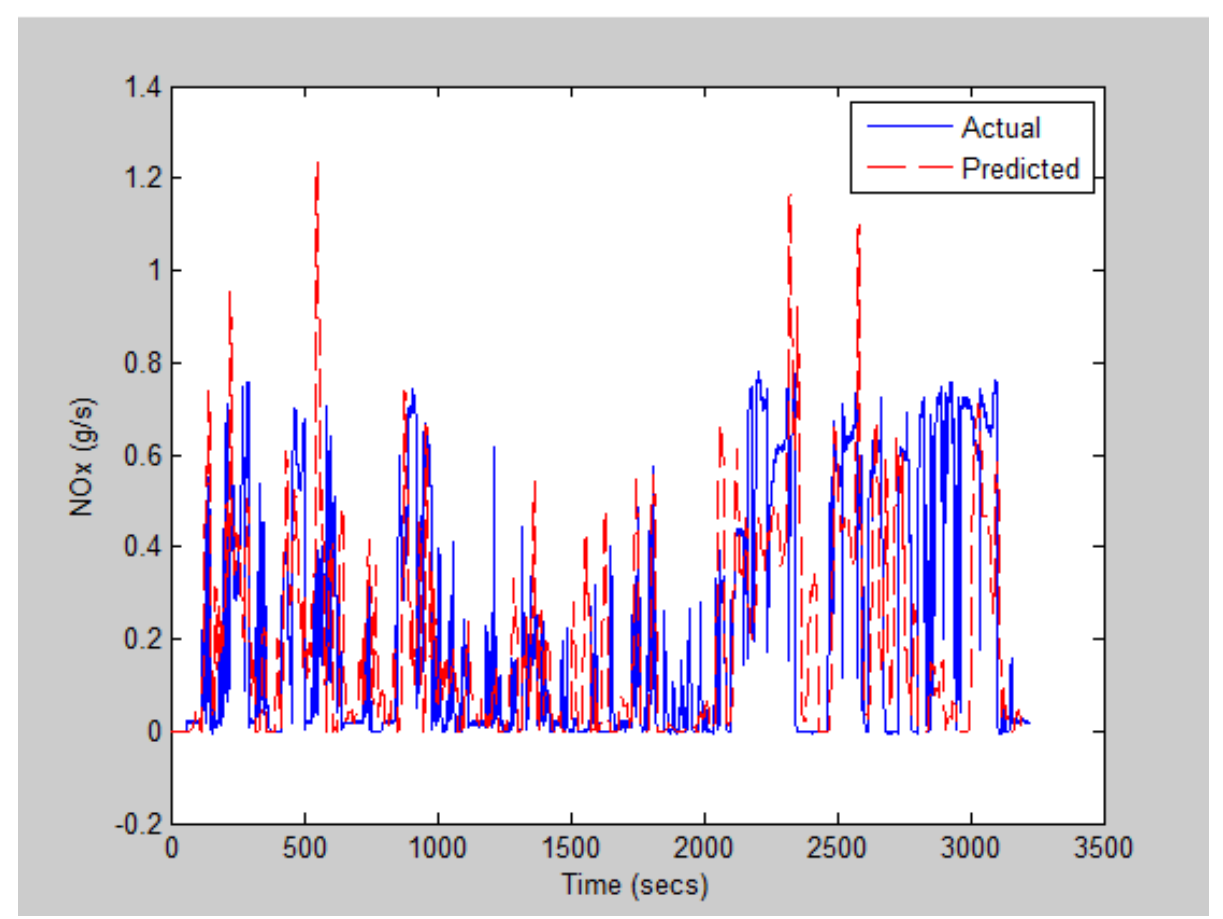

Figure 6.8.3: Predicted and Measured NOx Emissions for Washington, PA route When the ANN Was Trained with Data from Washington, PA 2 Route

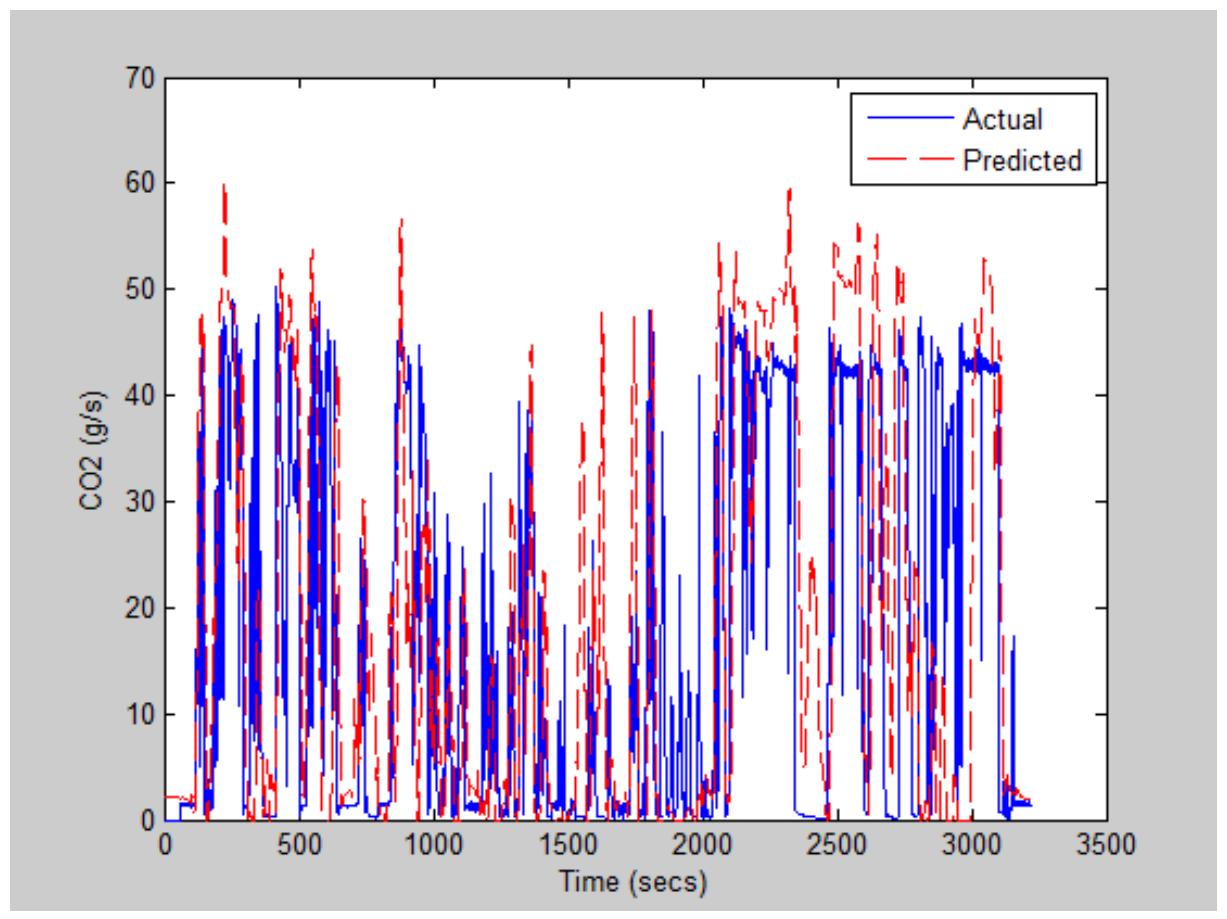

Figure 6.8.4: Predicted and Measured NOx Emissions for Washington, PA route When the ANN Was Trained with Data from Washington, PA 2 Route 
It was determined that the ANN was able to predict the emissions associated with a different engine than which it was trained. It is suggested that the ANN be trained with data from an engine of higher or equivalent power rating, compared to the engine which is sought to be modeled. When the ANN was trained with an engine with a lower power rating than the one being modeled, the differences between the predicted and measured values were greater due to the ANN extrapolating data beyond that with which it was trained.

\subsection{Summary of Model Verification}

In order to demonstrate the accuracy of the artificial neural network model a confidence interval analysis was preformed. The percent difference between the ANN model output and experimentally obtained data was analyzed. Equation 6.9.1 was used to determine the maximum error, which was a function of the standard deviation $(\sigma)$, number of samples $(n)$, and confidence level $\left(\mathrm{Z}_{\mathrm{a} / 2}\right)$. Equation 6.9.2 depicts the confidence interval. For a 95 percent confidence level the value for $\mathrm{Z}_{\mathrm{a} / 2}$ was 1.96 [56]. The confidence interval implied that $95 \%$ of the data will occur in the calculated range, or in other words there is $95 \%$ confidence that the percent difference between the emissions predicted by the ANN and the measured emissions will be in the range

presented. Table 6.9.1 displays confidence intervals associated with the predictions made by the ANN when data associated with the $400 \mathrm{hp}$ engine from Manufacturer A. The confidence intervals for various training and verification combinations are displayed in the table. It was shown that when the emissions module of the ANN is trained with Bruceton cycle data, and the vehicle module of the ANN also trained with data from the Bruceton Mills, WV route that the $95 \%$ confidence interval associated with NOx ranged from $3.6 \%$ to $7.8 \%$.

$$
\begin{aligned}
& \epsilon=Z_{\frac{\alpha}{2}} \frac{\sigma}{\sqrt{n}} \\
& \bar{x}-\epsilon<\mu<\bar{x}+\epsilon
\end{aligned}
$$

Equation 6.9.1

Equation 6.9.2 
Table 6.9.1: Confidence Interval Summary for Manufacturer A $400 \mathrm{hp}$

\begin{tabular}{|c|c|c|c|c|}
\hline \multicolumn{5}{|c|}{ 95\% Confidence Interval } \\
\cline { 1 - 2 } 1995 Manufacturer A 400 hp \\
\cline { 1 - 2 } Training Data & ANN2 & Prediction Data & NOx (\%) & $\mathrm{CO}_{2}(\%)$ \\
\hline Bruceton In-Use & Bruceton Cycle & Bruceton & $3.6<\mu<7.8$ & $11.2<\mu<18.2$ \\
\hline $\begin{array}{c}\text { Washington, } \\
\text { In-Use }\end{array}$ & Bruceton Cycle & Bruceton & $5.8<\mu<13.8$ & $13.3<\mu<24.1$ \\
\cline { 1 - 2 } $\begin{array}{c}\text { Washington } \\
\text { In-Use }\end{array}$ & Washington Cycle & Bruceton \& Wash. & $8.1<\mu<17.3$ & $9.9<\mu<18.3$ \\
\cline { 1 - 2 } $\begin{array}{c}\text { Washington } \\
\text { In-Use }\end{array}$ & Bruceton Cycle & Bruceton \& Wash. & $13.5<\mu<21.5$ & $9.9<\mu<18.5$ \\
\hline
\end{tabular}

The confidence intervals associated with the $350 \mathrm{hp}$ engine from Manufacturer A, and the 2002 engine from Manufacturers B and C are displayed in Table 6.9.2. These confidence intervals represent all routes and training data combinations which were examined. The data for the 2002 engines resulted from both modules of the ANN being trained with in-use data from that same engine. It was determined that $95 \%$ of the $\mathrm{CO}_{2}$ emissions predicted for the $1995350 \mathrm{hp}$ engine were in the range between 2.6 and 6.8 percent different from the measured emissions values. The confidence interval of percent differences for the NOx emissions from the $1995350 \mathrm{hp}$ engine ranged from $13.2 \%$ to $19.6 \%$. The $95 \%$ confidence intervals associated with the difference between predicted and measured emissions for the 2002 engines are also displayed in the table shown below.

Table 6.9.2: Confidence Interval Summary for Manufacturer A $350 \mathrm{hp}$, Manufacturer B, and Manufacturer C

\begin{tabular}{|c|c|c|c|c|c|}
\hline \multicolumn{7}{|c|}{ 95\% Confidence Interval } \\
\hline 1995 Manufacturer $\mathbf{A} 350 \mathrm{Hp}$ & \multicolumn{2}{|c|}{$\mathbf{2 0 0 2}$ Manufacturer $\mathbf{B}$} & \multicolumn{2}{|c|}{$\mathbf{2 0 0 2}$ Manufacturer $\mathbf{C}$} \\
\hline $\mathrm{NO} \times(\%)$ & $\mathrm{CO}_{2}(\%)$ & $\mathrm{NO}(\%)$ & $\mathrm{CO}_{2}(\%)$ & $\mathrm{NO} \times(\%)$ & $\mathrm{CO}_{2}(\%)$ \\
\hline $13.2<\mu<19.6$ & $2.6<\mu<6.8$ & $7.4<\mu<17.2$ & $3.1<\mu<10.9$ & $2.8<\mu<13.0$ & $0.6<\mu<4.8$ \\
\hline
\end{tabular}

Overall, the percent differences between the measured and predicted emissions displayed in the above tables are the same orders of magnitude of the coefficients of variation between measured emissions of the some route, discussed earlier in this document. For example, the COV in measured NOx values ranged from $3.4 \%$ to $11.1 \%$ for the routes examined, and the confidence intervals spanned ranges of $4.2 \%$ to $9.2 \%$ difference for NOx. This shows that with $95 \%$ 
confidence the emissions for NOx could be predicted within the variation in measured emissions between runs. 


\section{Significance}

The research in this document is unique because current emissions models do not account for road grade conditions, and previous research has determined them to be inaccurate. Currently there is not a standard means by which to accurately predict in use emissions based on engine dynamometer data for a particular heavy duty diesel engine. It is important to be able to predict in-use emissions due to the cost and labor required to test a currently employed engine on an engine dynamometer. Even though newly produced engines must meet stringent EPA emissions standards, these standards do not affect engines produced previously. Since numerous trucks and busses operate with engines older than those that the current standards apply, it is important to have a method of predicting their emissions in order to obtain an accurate emissions inventory. The model developed in this work can be employed as a tool to better the emissions prediction ability of current emissions inventory models. By employing the engine dynamometer data to develop the model, a result can be reached without any additional testing, which is economically beneficial from both a time and financial standpoint. As more in-use data becomes available, the model will become more applicable to a wider variety of driving scenarios.

\subsection{Impact of Road Grade}

In order to show the importance of the consideration of road grade in the prediction of emissions, the ANN model was used to predict emissions without taking into account road grade. The results shown in the following table were determined when road grade was removed as an input to the ANN. The vehicle module of the ANN was retrained with only the inputs relating to engine torque and vehicle speed, and when was employed to predict the emissions associated with various routes. Table 7.1.1 shows the percent difference between measured and predicted emissions when road grade is not employed as an input into the model. Various combinations of training and verification data were examined. The emissions were predicted for the Bruceton Mills, WV to Sabraton, WV route when ANN1 was trained with Bruceton Mills, WV to Sabraton, WV in-use data, and ANN2 was trained both with data from the Bruceton Mills, WV Cycle and the Washington, PA Cycle. The emissions associated with the route from Sabraton, WV to Bruceton Mills, WV were also predicted without considering road grade. The emissions

for the Sabraton, WV to Bruceton Mills, WV route were predicted twice, in both cases ANN1 was trained with data from the Washington, PA2 route. ANN2 was trained for one case with 
data from the Bruceton Mills, WV cycle, and for another case with data from the Washington, PA1 cycle. The variations in the percent differences between predicted and measured NOx and $\mathrm{CO}_{2}$ between runs was due to reinitializing the weights of the ANN. With each run the initial weights associated with the emissions module of the ANN were reinitialized, resulting in different starting values, and a different final convergence point.

It was determined that when road grade was not accounted for the percent difference between the measured and predicted emissions was greater than the difference when road grade was considered as an input to the ANN. For example, when road grade was not considered the average percent difference between the predicted and measured NOx emissions for the Bruceton Mills, WV to Sabraton, WV route was $38.2 \%$, and when road grade was considered the difference was 5.8\%. The percent difference between measured and predicted $\mathrm{CO}_{2}$ emissions when road grade was not considered was $36.4 \%$, while when road grade was considered the difference was $12.6 \%$. Similar differences in the average percent differences with and without accounting for road grade were observed for all of the training and verification combinations displayed in the table below.

The increase in accuracy when the ANN was provided with road grade data indicated that accounting for road grade resulted in a model that is capable of predicting $\mathrm{NOx}$ and $\mathrm{CO}_{2}$ emissions more accurately than a model that does not account for the effects of road grade. 
Table 7.1.1: Emissions Predicted Without Road Grade as an Input Compared to When Road Grade was an Input

\begin{tabular}{|c|c|c|c|}
\hline \multicolumn{4}{|c|}{ ANN 1 Trained with Bruceton2Sab 2 Data } \\
\hline \multicolumn{4}{|c|}{ ANN 2 Trained with Sabraton to Bruceton Mills Data } \\
\hline Verification Data & Run & $\%$ Diff. NOx & $\%$ Diff. $\mathrm{CO}_{2}$ \\
\hline \multirow{4}{*}{ Bruceton2Sab 1} & 1 & 40.9 & 38.4 \\
\hline & 2 & 36.6 & 35.8 \\
\hline & 3 & 37.1 & 35.1 \\
\hline & Average & 38.2 & 36.4 \\
\hline \multicolumn{4}{|c|}{ ANN 1 Trained with Bruceton2Sab 2 Data } \\
\hline \multicolumn{4}{|c|}{ ANN 2 Trained with Washington, PA 1 Data } \\
\hline Verification Data & Run & $\%$ Diff. NOx & $\%$ Diff. $\mathrm{CO}_{2}$ \\
\hline \multirow{4}{*}{ Bruceton2Sab 1} & 1 & 39.2 & 38.3 \\
\hline & 2 & 34.4 & 28.4 \\
\hline & 3 & 32.5 & 30.0 \\
\hline & Average & 35.4 & 32.2 \\
\hline \multicolumn{4}{|c|}{ ANN 1 Trained with Washington, PA2 1 Data } \\
\hline \multicolumn{4}{|c|}{ ANN 2 Trained with Sabraton to Bruceton Mills Data } \\
\hline Verification Data & Run & $\%$ Diff. NOx & $\%$ Diff. $\mathrm{CO}_{2}$ \\
\hline \multirow{4}{*}{ Sab2Bruceton 1} & 1 & 43.7 & 31.2 \\
\hline & 2 & 56.2 & 35.5 \\
\hline & 3 & 42.0 & 30.0 \\
\hline & Average & 47.3 & 32.2 \\
\hline \multicolumn{4}{|c|}{ ANN 1 Trained with Washington, PA2 1 Data } \\
\hline \multicolumn{4}{|c|}{ ANN 2 Trained with Washington, PA 1 Data } \\
\hline Verification Data & Run & $\%$ Diff. NOx & $\%$ Diff. $\mathrm{CO}_{2}$ \\
\hline \multirow{4}{*}{ Sab2Bruceton 1} & 1 & 25.6 & 52.0 \\
\hline & 2 & 35.6 & 52.1 \\
\hline & 3 & 50.4 & 38.7 \\
\hline & Average & 37.2 & 47.6 \\
\hline
\end{tabular}




\section{Recommendations and Conclusions}

\subsection{Conclusions}

An ANN model was developed to predict heavy duty diesel engine emissions, employing engine dynamometer data, data acquired through in-use testing, and took into account the affects of road grade. The ANN employed the unique inputs of data that had been pre-processed with two moving averages, each incorporating a different number of points.

Initially data obtained from the FTP engine dynamometer cycles was employed to train the ANN, however it was determined that the FTP cycle did not provide adequate information to train the emissions module of the ANN. One of the concerns with data obtained from FTP cycle testing was that engines of certain model years were equipped with defeat devices, resulting in the emissions produced in-use exceeding the emissions produced during FTP testing. Rather than employing the FTP cycle, engine dynamometer cycles that were designed to simulate the Bruceton Mills, WV route and the Washington, PA route were used.

It was determined that when the vehicle module of the ANN was trained with in-use data from the Bruceton Mills, WV route, and the emissions module of the ANN was trained with engine dynamometer data from the cycle designed to simulate the Bruceton Mills, WV route, the ANN was able to predict NOx within $6 \%$ of the measured values. The average difference between the measured and predicted $\mathrm{CO}_{2}$ values for the same training and verification scenario mentioned above was less than $15 \%$. It was also demonstrated that the ANN was able to predict emissions that associated with routes that differ from those by which it was trained. When the ANN was trained with in-use data from the Washington, PA route it was able to predict the $\mathrm{NOx}$ and $\mathrm{CO}_{2}$ emissions with percent differences from the measured values of $20 \%$ or less.

It was also shown that the ANN was able to predict emissions associated with a different engine than that which it was trained, if the different engine was of a lower horsepower than the engine that produced the training data. The ANN was able to predict emissions with percent differences ranging from $13 \%$ to $19.6 \%$ for $\mathrm{NOx}$ and $2.6 \%$ to $6.8 \%$ for $\mathrm{CO}_{2}$ with $95 \%$ confidence. 
It was also demonstrated that the ANN could predict emissions when both the vehicle and the emissions module were trained with in-use data. The emissions associated with two separate 2002 engines from different manufacturers were modeled using the ANN. It was determined that when in-use data from the 2002 engines was used to train the ANN it was able to predict the NOx emission with an average of less than $2.5 \%$ difference from the measured values for certain routes.

A variety of routes were used for training and verification of the ANN, and it was determined that the ANN was capable of predicting emissions of $\mathrm{NOx}$ and $\mathrm{CO}_{2}$ within $20 \%$ or less of the measured values. An analysis of the in-use training data showed an average $\mathrm{COV}$ in $\mathrm{CO}_{2}$ measurements of up to $4.6 \%$ for certain routes for the $400 \mathrm{hp}$ engine from Manufacturer A. The COV for the NOx emissions associated with the same engine was as high as $11.11 \%$ for a certain route. The variance between measured emission values on repeated tests of the same route was also examined for an engine from another manufacturer, and it was determined that an average $\mathrm{COV}$ for all of the routes examined was $28.4 \%$ for $\mathrm{CO}_{2}$, and $36.8 \%$ for NOx. Seeing as the data from these in-use tests were employed as training and verification data for the ANN, it should be noted that the percent differences between that measured and the predicted emissions values are on the order of the variance in measured emissions between tests.

It was also shown that a method of compensating for the weight difference between the vehicle from which training data was acquired and the vehicle being modeled was developed. It was determined that the training data should be obtained from a vehicle weighing more or equivalent to the vehicle being modeled.

In-use emissions data will continue to become more available as it is currently a requirement by the US EPA. It has been shown that the ANN developed in this work is able to better predict emissions when trained with in-use emissions data, therefore as more in-use data become available the ANN has the potential to be applied to a wider variety of engines.

Rather than replace current emissions inventory estimations models, it was recommended that the model presented in this work be employed to aid in the prediction of emissions in the current 
models. In order for the work presented in this dissertation to be used to predict emissions inventories, additional work would be required, such as acquiring climate data for the regions for which inventories would be determined. Also, route information would be needed for all of the routes being included in the emissions inventory.

\subsection{Recommendations for Future Work}

It is recommended that future work be conducted that would result in data which could be employed to determine the affects of deterioration and ambient conditions on the produced emissions. The data available for this analysis was acquired via in-use testing, and therefore variation in conditions made it impossible to distinguish between ambient and deterioration effects on the emissions. It is recommended that engines be tested in a test cell environment where conditions can be controlled in order to acquire data that would allow the impacts of deterioration and ambient conditions to be examined.

The recommendation is also made that as more in-use data becomes available for present technology engines that the ANN be trained with that data and employed to predict data associated with engines that are currently in production. 


\section{References}

1. Merrion, D.F., "Heavy Duty Diesel Emission Regulations - Past, Present, and Future," SAE Paper. 2003-01-0040. 2003.

2. $\quad$ "Diesel Emissions Health and Environmental Effects." Maryland State. Web. 12 July 2010. <http://www.mde.state.md.us/airprograms/modbile_sources/dieselemmiscontrol>.

3. Public Health and Environmental Impacts of Diesel Emissions. West Coast Collaborative, 2005.

4. Environmental Protection Agency. Diesel Exhaust In The United States. US EPA, 2002.

5. Health Effects of Diesel Exhaust. California: American Lung Association.Web. 13 July 2010. <http://www.californialung.org $>$.

6. Tatur, M., Laermann, M., Koehler, E., Tomazic, D., Holland, T., Robinson, D., Dowell, J. and Price, K., "Development of an Emissions Control Concept for an IDI Heavy-Duty Diesel Engine Meeting 2007 Phase-In Emissions Standards," SAE Paper. 2007-01-0235. 2007.

7. Kean, A.J., Sawyer, R.F., and Harley, R.A. "A fuel-based assessment of off-road diesel engine emissions." Journal of the Air and Waste Management Association. 50:19291939, 2007.

8. McCormick, R., Ryan, L., Daniels, T., Yanowitz, J., and Graboski, M., "Comparison of Chasis Dynamometer In-Use Emissions with Engine Dynamometer FTP Emissions for Three Heavy-Duty Diesel Vehicles," SAE Paper. 982653. 1998.

9. Dieselnet Emissions Standards: Heavy Duty Truck and Bus Engines. United States: Dieselnet. Web. 13 July 2010. <http://www.dieselnet.com>.

10. Krijnsen, H.C., Van Kooten, WE J., Calis, HP A., Verbeek, R. P., and Van Den Bleek, C. M. "Evaluation of an Artificial Neural Network for NOx Emission Prediction from a Transient Diesel Engine as a Base for NOx Control." The Canadian Journal of Chemical Engineering 78. April: 408-17, 2000.

11. Clark, N., Tehranian, A., Jarrett, R., and Nine, R., "Translation of Distance-Specific Emissions Rates between Different Heavy Duty Vehicle Chassis Test Schedules," SAE Paper. 2002-01-1754. 2002.

12. Ramamurthy, R., Clark, N., Atkinson, C., and Lyons, D., "Models for Predicting Transient Heavy Duty Vehicle Emissions," SAE Paper. 982652. 1998. 
13. Joumard, R., Philippe, F., and Vidon, R., "Reliability of the Current Models of Instantaneous Pollutant Emissions." The Science of the Total Environment 235: 133-142, 1999.

14. California Air Resources Board. EMFAC2001/EMFAC2002. Calculating Emissions Inventories for Vehicles in California, User's Guide, 2002.

15. Bachman, W., "A GIS-Based Modal Model of Automobile Exhaust Emissions." US EPA Research and Development: 1-4.1998.

16. "Description and History of the MOBILE Highway Vehicle Emission Factor Model," U.S. Environmental Protection Agency Office of Transportation and Air Quality, Feb. 2004. Web. 12 July 2010. <http://www.epa.gov/oms/models/mob_hist.txt>.

17. EPA Releases MOVES2010 Mobile Source Emissions Model: Questions and Answers. Rep. no. EPA-420-F-09-073. US EPA, 2009.

18. Yanowitz, J., Graboski, M., and McCormick, R., "Prediction of In-Use Emissions of Heavy Duty Diesel Vehicles from Engine Testing," Environmental Science \& Technology. 36:270-275, 2002.

19. Singh, R. B., Huber, A. H., and Braddock, J.N., "Development of a Microscale Emission Factor Model for Particulate Matter for Predicting Real-Time Motor Vehicle Emissions," Air \& Waste Management Association 53: 1204-1217, 2003.

20. Ubanwa, B., Burnette, A., Kishan, S., and Fritz, S.G.,"Exhaust Particulate Matter Emission Factors and Deterioration Rate for In-Use Motor Vehicles," ASME Paper. 125:513-523, 2003.

21. Shah, S. D., Kent C. Johnson, J. Miller, W., and Crocker, D.R. III. "Emission Rates of Regulated Pollutants From On-Road Heavy-Duty Diesel Vehicles," Atmospheric Environment 40: 147-53. 2006.

22. Brown, J., King, F., Mitchell, W., Squier, W., Harris, B., and Kinsey, J., "On-Road Facility to Measure and Characterize Emissions from Heavy-Duty Diesel Vehicles," Air \& Waste Management Association. 52:388-395, 2002.

23. Weaver, C., Turner, S., and Balam-Almanza, M., "Comparison of In-Use Emissions from Diesel and Natural Gas Trucks and Buses," SAE Paper. 2000-01-3473. 2000.

24. Clark, N., Gajendran, and P., Kern, J., "A Predictive Tool for Emissions from HeavyDuty Diesel Vehicles," Environmental Science \& Technology. 37:7-15, 2003.

25. Ramamurthy, R. and Clark, N., "Atmospheric Emissions Inventory Data for Heavy-Duty Vehicles," Environmental Science \& Technology. 33:55-62, 1999. 
26. Barth, M., Feng A., Norbeck, J., and Ross, M., "Model Emissions Modeling: A Physical Approach," Transportation Research Record 1520: 81-88.

27. Jost, P., Hassel, D., and Sonnborn, K. S., "A New Method to Determine Exhaust Emission Factors for Heavy Duty Vehicles," The Science of the Total Environment 169: 213-217, 1995.

28. Yanowitz, J., McCormick, R., and Graboski, M., "In Use Emissions from Heavy-Duty Diesel Vehicles," Environmental Science \& Technology. 34:729-740, 2000.

29. Antonacci, G., Todeschini, I., and Cemin, A., Influence of Road Gradient on Emissions Factors. Proc. of 21st TFEIP Meeting, Milan, 2008.

30. Yanowitz, J., Graboski, M., Ryan, L., Alleman, T., and McCormick, R., "Chassis Dynamometer Study of Emissions from 21 In-Use Heavy-Duty Diesel Vehicles," Environmental Science and Technology. 33:209-213. 1999.

31. Zachariadis, T., Ntziachristos, L., and Samaras, Z., "The Effect of Age and Technological Change on Motor Vehicle Emissions," Transportation Research Part D 6: 221-227, 2001.

32. McDonald, Gary C., "Confidence Intervals for Vehicle Emission Deterioration Factors," Technometrics 23.3: 239-242, 1981.

33. Ntziachristos, L., and Samaras, Z., "An Empirical Method for Predicting Exhaust Emissions of Regulated Pollutants From Future Vehicle Technologies," Atmospheric Environment 35: 1985-1999, 2001.

34. Carey, P. and Lorang, P., Overview of Methodology for Tier O In-Use Deterioration and Key Issues for Comment. Rep. no. M6.EXH.008. US EPA, 1999.

35. Khan, ABM. Route and Grade Sensitive Modeling of Fuel Efficiency and Emissions for Diesel Buses. Diss. West Virginia University, 2009.

36. Perhinschi, M., Artificial Intelligence Techniques in Engineering. Ch. 1. Handout 1. Morgantown: West Virginia University, 2008.

37. Alonso, J. M., Alvarruiz F., Desantes, J. M., Hernandez, L., Hernandez, V., and Molto, G., "Combining Neural Networks and Genetic Algorithms to Predict and Reduce Diesel Engine Emissions," IEEE Transactions on Evolutionary Computation 11.1: 778-81, 2007.

38. Hempel, D., "The Fractal Nature of Consciousness, Page 1."AboveTopSecret.com: Conspiracy Theories, UFOs, Politics, and Many Other "Alternative Topics" 1' July 2010. Web. 13 July 2010. <http://www.abovetopsecret.com/forum/thread532721/pg1>. 
39. "The Use of Self-Organizing Maps in Recommender Systems," MOVSOM Research Lab. Web. 13 July 2010. <http://rslab.movsom.com/paper/somrs/html/chapter3.html $>$.

40. Perhinschi, M., Artificial Intelligence Techniques in Engineering (Artificial Neural Networks). Ch. 4. Handouts. 12-17. Morgantown: West Virginia University, 2008.

41. Tutuncu, K. and Allahverdi, N., "Modeling the Performance and Emission Characteristics of Diesel Engine and Petrol-Driven Engine by ANN." CompSysTech III.: 1-6, 2009.

42. Obodeh, O. and Ajuwa, C.I., "Evaluation of Artificial Neural Network Performance in Predicting Diesel Engine NOx Emissions," European Journal of Scientific Research. 33: 642-653, 2009.

43. Arcaklioglu, E. and Celikten, I., "A Diesel Engine's Performance and Exhaust Emissions," Applied Energy Journal. 80:11-22, 2005.

44. Obodeh, O. and Ajuwa, C.I., "Calibration of Aging Diesel Engine with Artificial Neural Networks," European Journal of Scientific Research. 24: 520-531, 2008.

45. Galindo, J., Lujan J. M., Serrano J. R., and Hernandez, L., "Combustion Simulation of Turbocharger HSDI Diesel Engines During Transient Operation Using Neural Networks," Applied Thermal Engineering 25: 877-98, 2004.

46. Xu, K., Xie, M., Tang, L.C., and Ho, S.L., "Application of Neural Networks in Forecasting Engine Systems Reliability,” Applied Soft Computing 2: 255-268, 2003.

47. Desantes, J. M., Benajes J. V., Molina S. A., and Hernandez, L., "Multi-objective Optimization of Heavy-Duty Diesel Engines Under Stationary Conditions," Journal of Automobile Engineering 219: 77-87, 2004.

48. Parlak, A., Yasar I., Halit Y., and Egrisogut, L., "Application of Artificial Neural Network to Predict Specific Fuel Consumption and Exhaust Temperature for a Diesel Engine," Applied Thermal Engineering 26: 824-28, 2006.

49. Delagrammatikas, G. J. and Assanis, D. N., "Development of a Neural Network Model of Advanced, Turbocharged Diesel Engine for Use in Vehicle-Level Optimization Studies," Proc. Instn Mech. Engrs 218: 521-533, 2004.

50. Hashemi, N. and Clark, N. N., "Artificial Neural Network as a Predictive Tool for Emissions From Heavy-Duty Diesel Vehicles in Southern California," Int. J. Engine Res. 321-336, 2007.

51. Traver, M. L., Atkinson, R. J., and Atkinson, C. M., "Neural Network-Based Diesel Engine Emissions Prediction Using In-Cylinder Combustion Pressure," SAE Technical Paper. 1999-01-1532:1-15. 1999. 
52. Brace, C., "Prediction of Diesel Engine Exhaust Emissions Using Artificial Neural Networks," Proc. of Neural Networks in Systems Design, Lucas Electrical and Electronic Systems, Solihull, 1-11, 1998.

53. Thompson, G. J., Atkinson C. M., Clark N. N., Long T. W., and Hanzevack, E. "Neural Network Modeling of the Emissions and Performance of a Heavy-Duty Diesel Engine," Proc. Instn. Mech. Engineers 214: 111-126, 2000.

54. Reynolds, A. W. and Broderick, B. M., "Development of an Emissions Inventory Model for Mobile Sources," Transportation Research Part D 5: 77-101, 2000.

55. "Powertrain System Analysis Toolkit (PSAT)." Argonne National Laboratory. Web. 18 January 2011.< http://www.transportation.anl.gov/modeling_simulation/PSAT/index. html $>$.

56. Madireddy, M. R., Methods for Reconstruction of Transient Emissions from Heavy Duty Vehicles. Diss. West Virginia University, 2008.

57. Aravelli, A., Real-Time Measurement of Oxides of Nitrogen from Heavy Duty Diesel Engines. Thesis. West Virginia University, 2003.

58. Hashemi, N., Effects of Artificial Neural Network Speed-Based Inputs on HeavyDutyVehicle Emissions Prediction. Thesis. West Virginia University, 2009.

59. Ramamurthy, R. Clark, N., Atkinson, C., and Lyons, D., "Models for Predicting Transient Heavy Duty Vehicle Emissions," SAE Paper. 982652. 1998.

60. Madireddy, M. and Clark, N., "Sequential Inversion Technique and Differential Coefficient Approach for Accurate Instantaneous Emissions Measurement," International Journal of Engine Research. June 2006.

61. Mechtly, E. A., The International System of Units, Physical Constants and Conversion Factors. NASA SP-7012. National Aeronautics and Space Administration, 1973.

62. Shade, B. C., A Performance Evaluation of the MEMS-An On-Road Emissions Measurement System Study. Thesis. West Virginia University, Morgantown, WV, 2000.

63. Gautam, M., Clark, N. N., Thompson, G.J., Carder, D.K., and Lyons, D.W., Development of In-Use Testing Procedures for Heavy Duty Diesel-Powered Vehicle Emissions. West Virginia University, Morgantown, WV, 2000.

65. Thompson, G., MEMS Routes Phase II Report. West Virginia University, Morgantown, WV. Personal Communications. 2011. 
66. Panchal, G., Ganatra, A., Shah, P., and Panchal, D., "Determination of Over-Learning and Over-Fitting Problem in Back Propagation Neural Network," International Journal on Soft Computing. May 2011:Vol 2.

67. Wayne, W., Perhinschi, M., Clark, N., Tamayo, S., and Tu, J., "Integrated Bus Information System (IBIS) - A Vehicle Procurement Resource for Transit," Transportation and Research Board Annual Meeting. 2011.

68. Kern, J., Inventory and Prediction of Heavy-Duty Diesel Vehicle Emissions. Thesis. West Virginia University, 2000.

69. Chapter 3 Artificial Neural Networks. Power Point Presentation. HCMC University of Technology, Sept. 2008.

70. Krishnamurthy, M., Characterization of In-Use Emissions from On-Highway Heavy-Duty Diesel Engines. Thesis. West Virginia University, Morgantown, WV, 2000.

71. Thompson, G., Gibble, J., Clark, N., and Gautam, M., "Influences of Real-World Conditions on In-Use Emission from Heavy-Duty Diesel Engines," SAE Paper. 2006-013393. 2006. 


\section{Appendix}

This appendix consists of the code employed to obtain the data presented in this document. The code is presented in four modules. The first module loads the training data into Matlab, and then pre-processes that data, creates the vehicle module of the ANN, and then trains the vehicle module ANN. The second module loads data into Matlab, pre-processes that data, and then creates the emissions module of the ANN, and then trains the emissions modules of the ANN. The third module loads verification data into the Matlab, pre-processes that data, and then applies the previously trained vehicle module of the ANN to the verification data. The forth module, employs the outputs of the third module, pre-processes those outputs, and applies the previously trained emissions module to the verification data.

The outputs of the forth module are the predicted $\mathrm{NOx}, \mathrm{CO}_{2}, \mathrm{HC}$, and $\mathrm{CO}$ emissions on a mass rate $(\mathrm{g} / \mathrm{s})$ basis. The structure of the ANN may be altered by changing variables in the first and second modules. Currently, the code is setup to employ in-use training and verification data that was obtained with $10 \mathrm{hz}$ sampling, and engine dynamometer training data that was obtained with $1 \mathrm{hz}$ sampling. If data is employed with different sampling rates it is recommended that the number of points in the moving average applied to the input data be altered in correlation to the sampling frequency. For example, data sets with a lower sampling frequency require fewer data points in the moving average. 


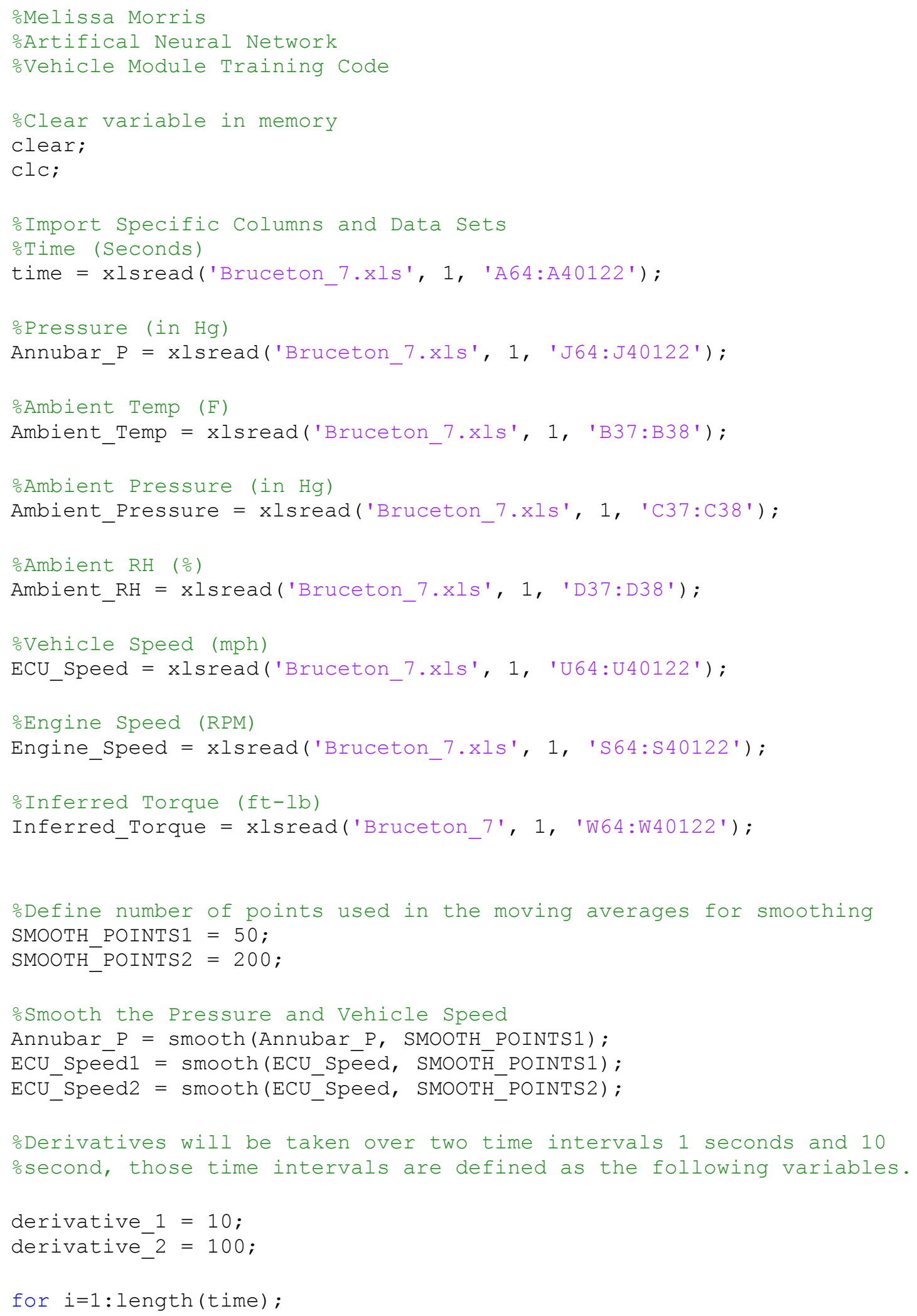




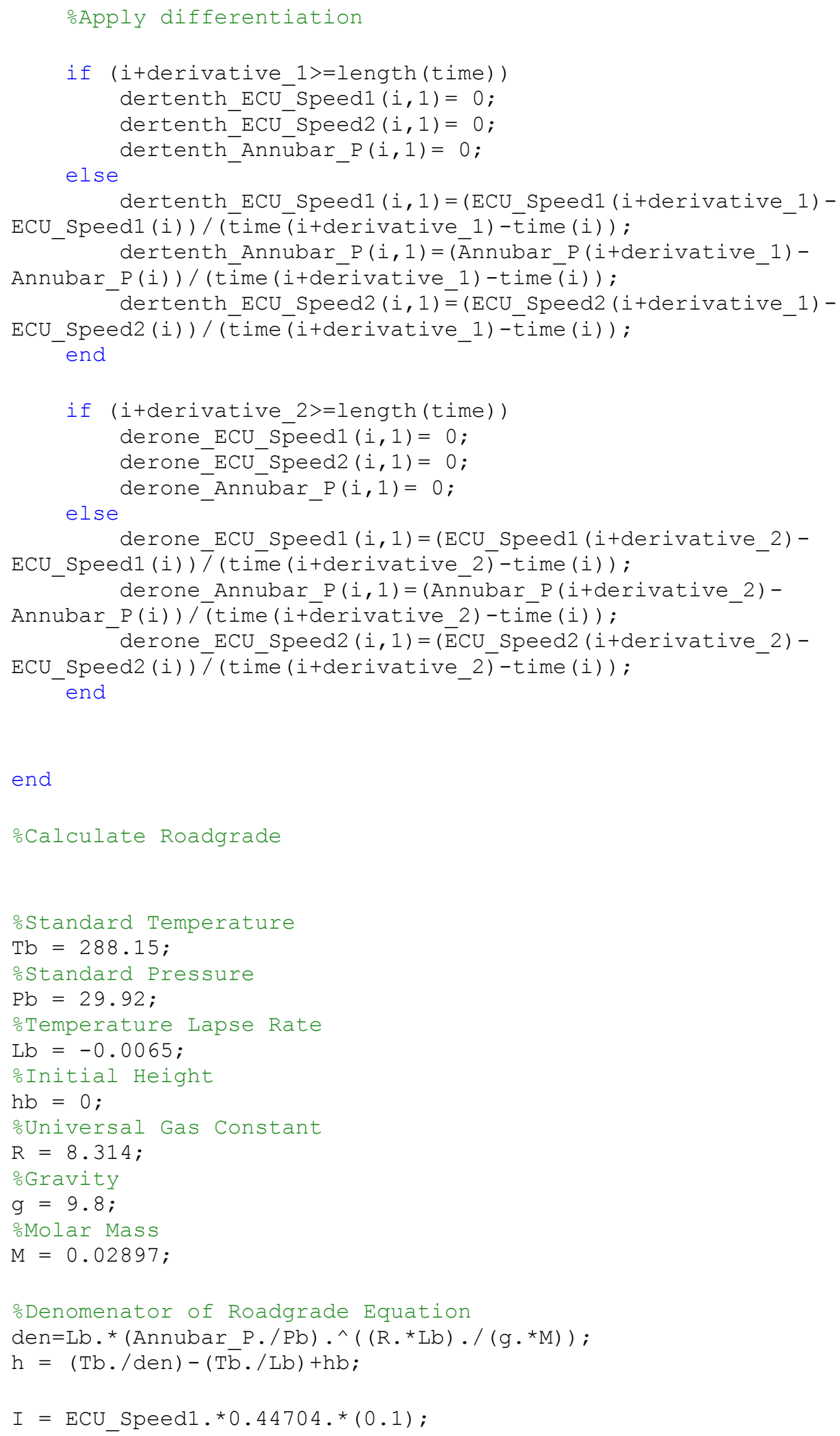




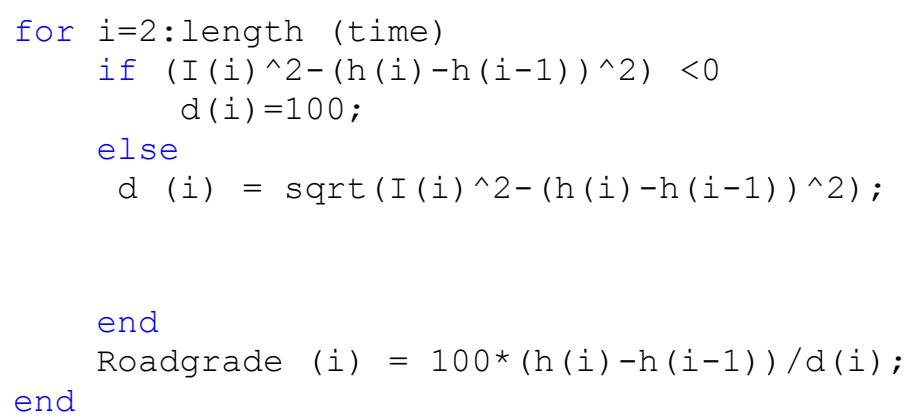




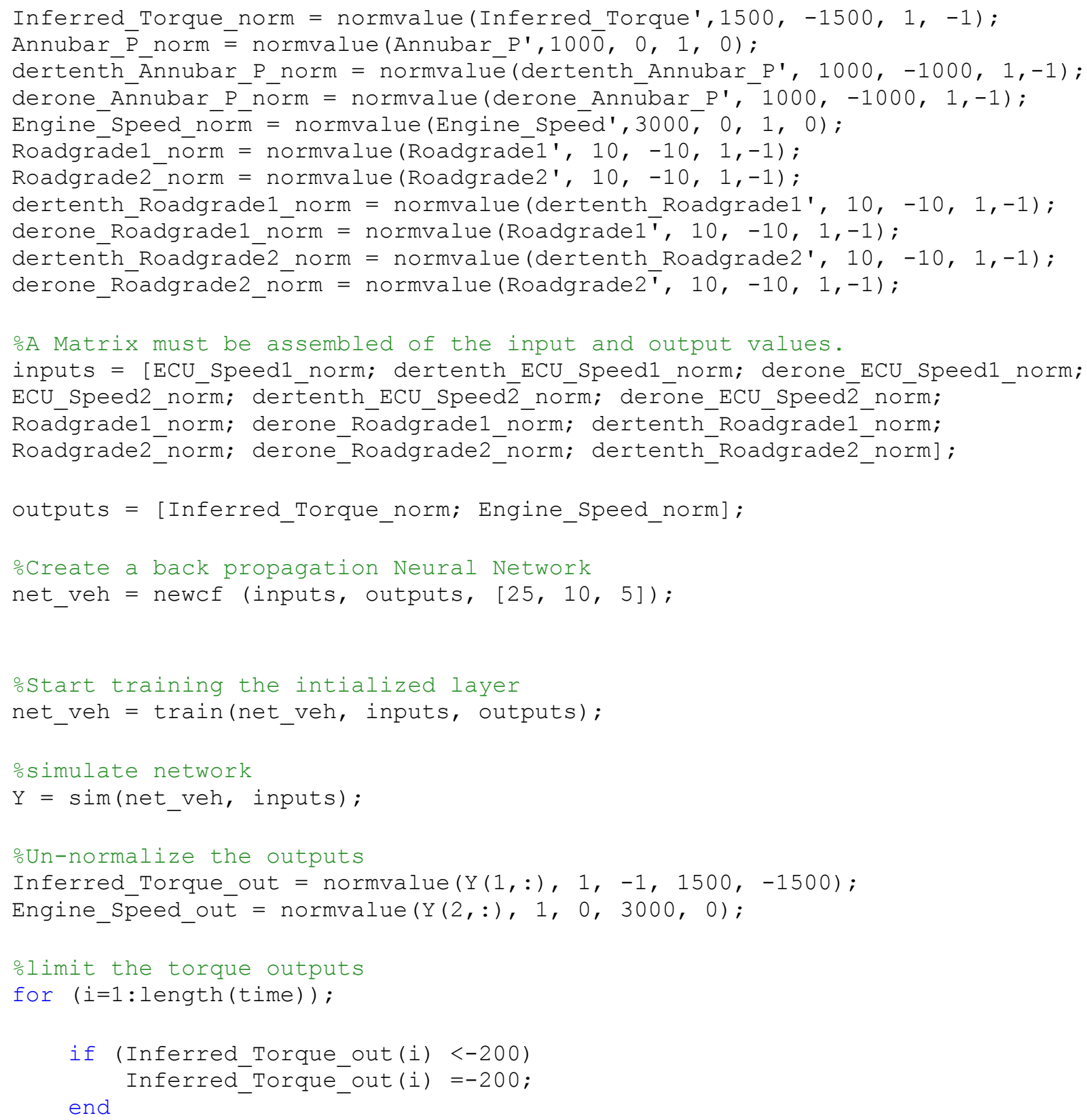


xlabel ('Time (secs)');

ylabel ('Engine Speed (RPM)') ;

axis ([0 max (time) -1000 3000]);

legend('Actual', 'Fit'); 
oMelissa Morris

oArtifical Neural Network

Emissions Module Training Code

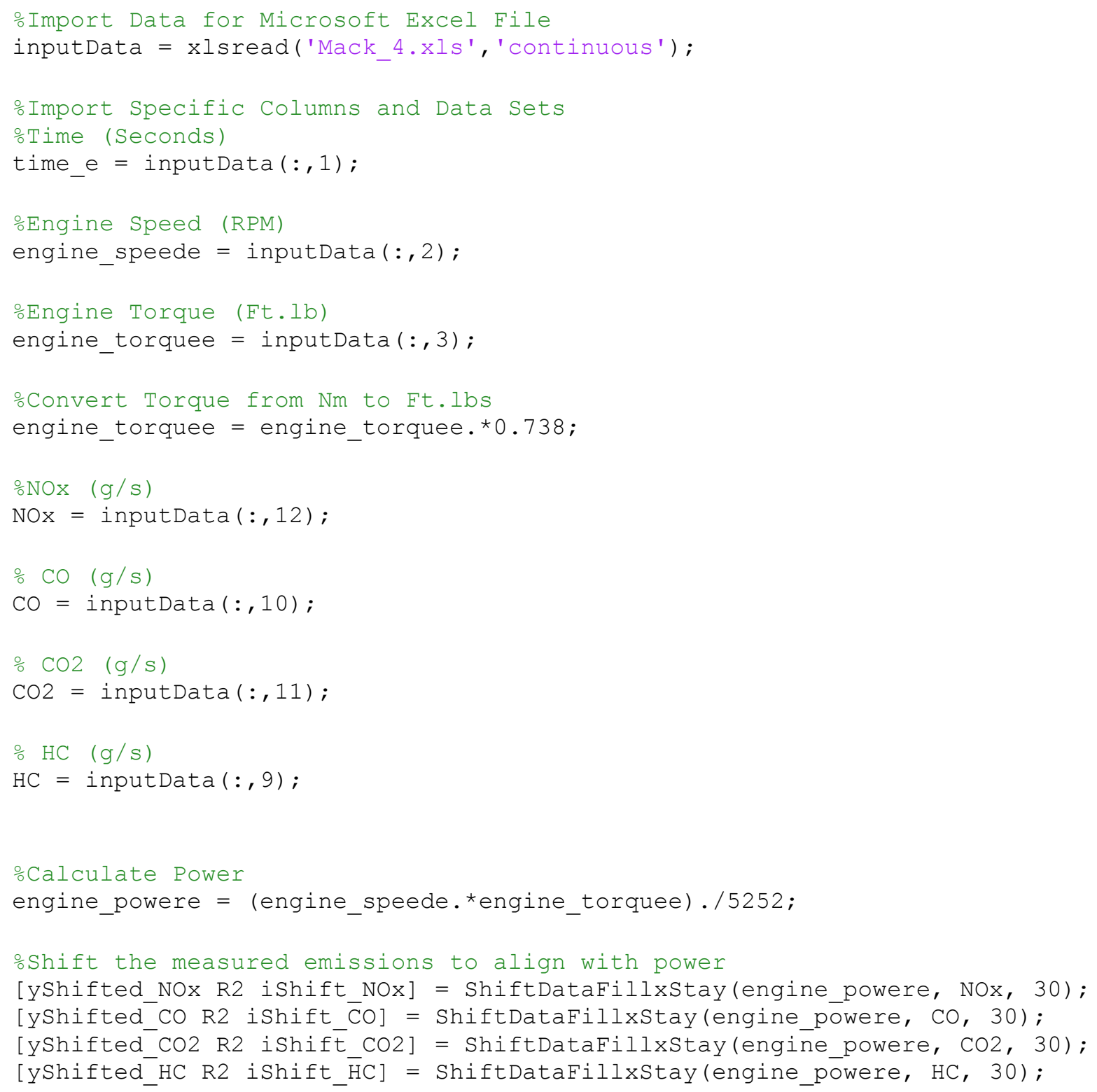




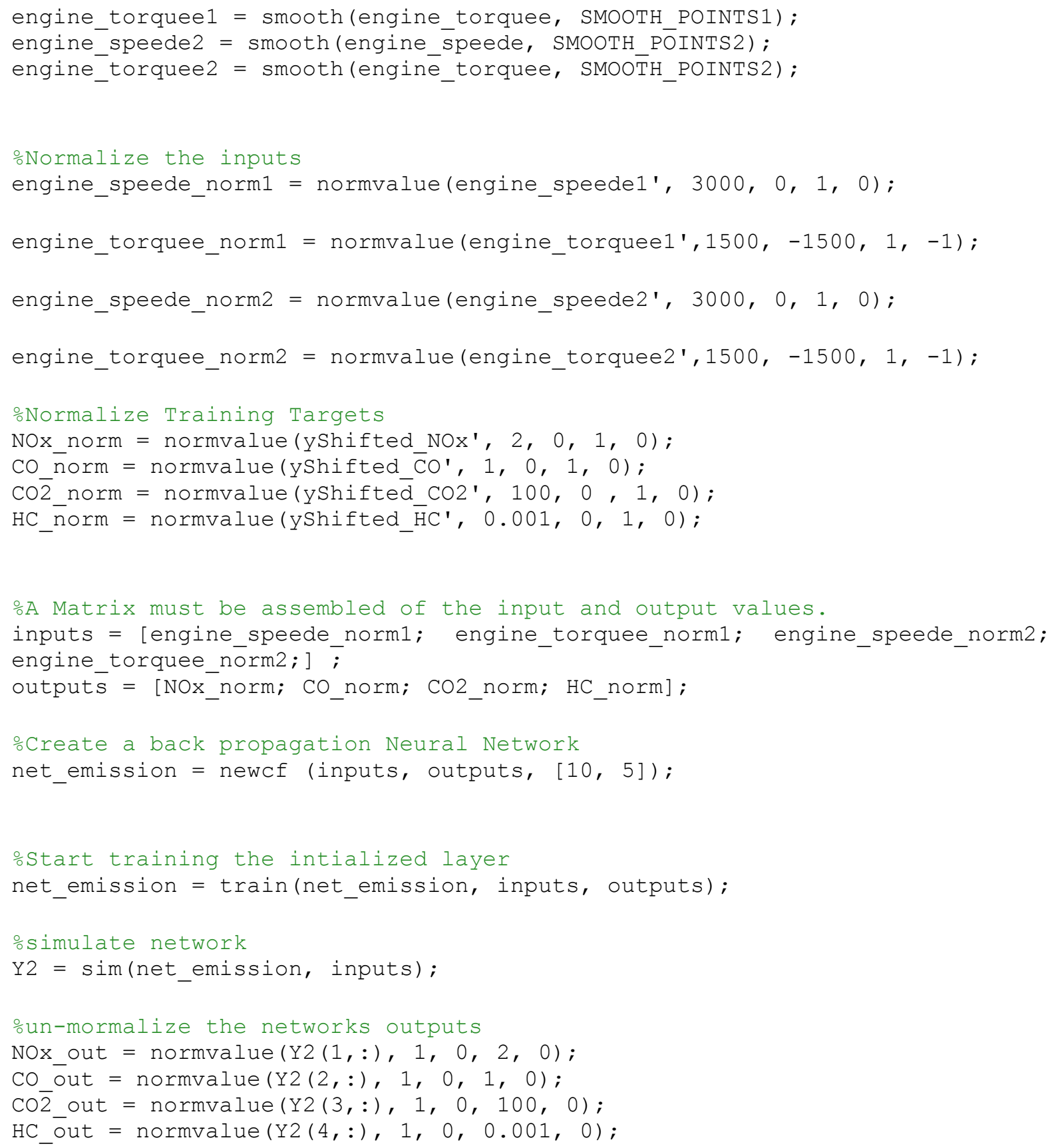




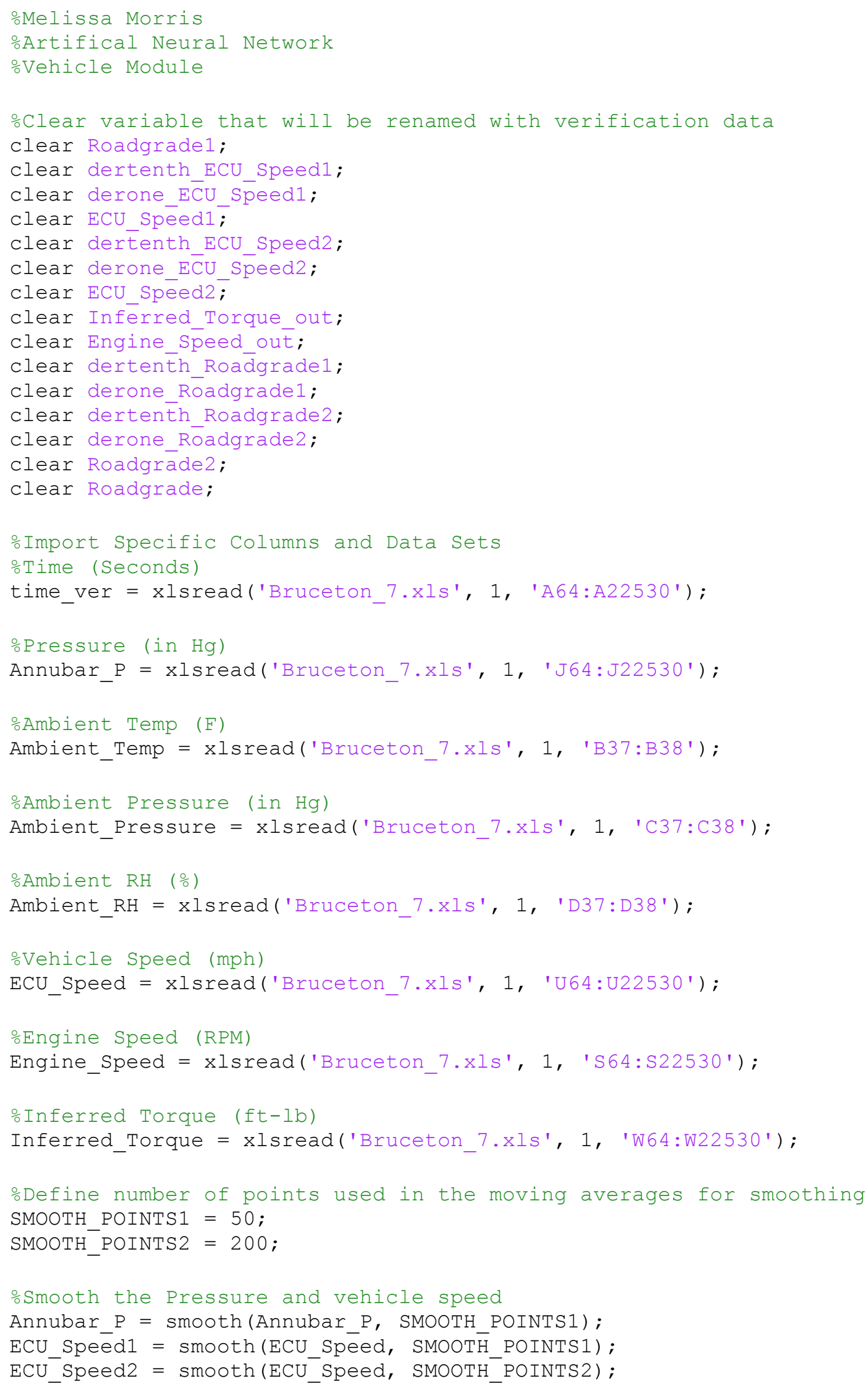




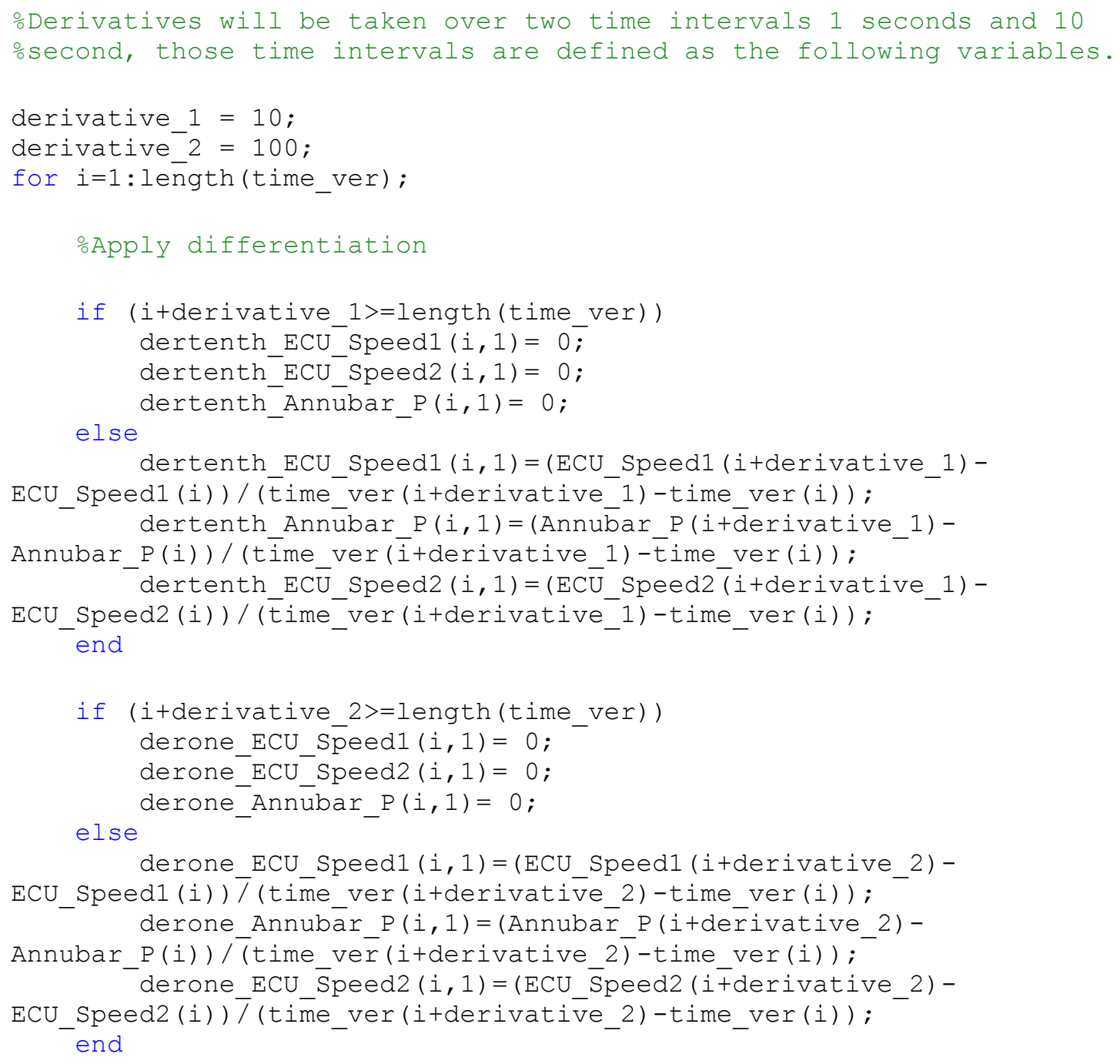

end

oCalculate Roadgrade

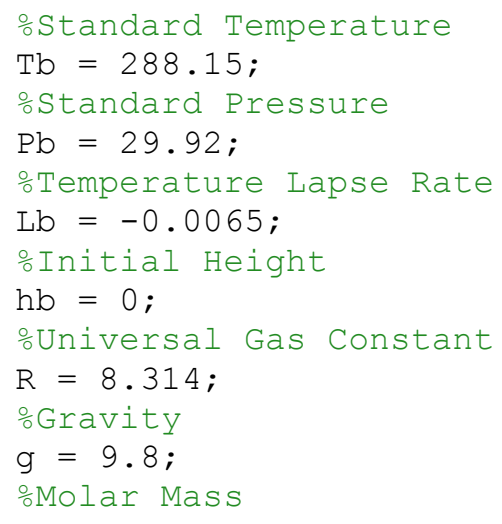


$\mathrm{M}=0.02897$;

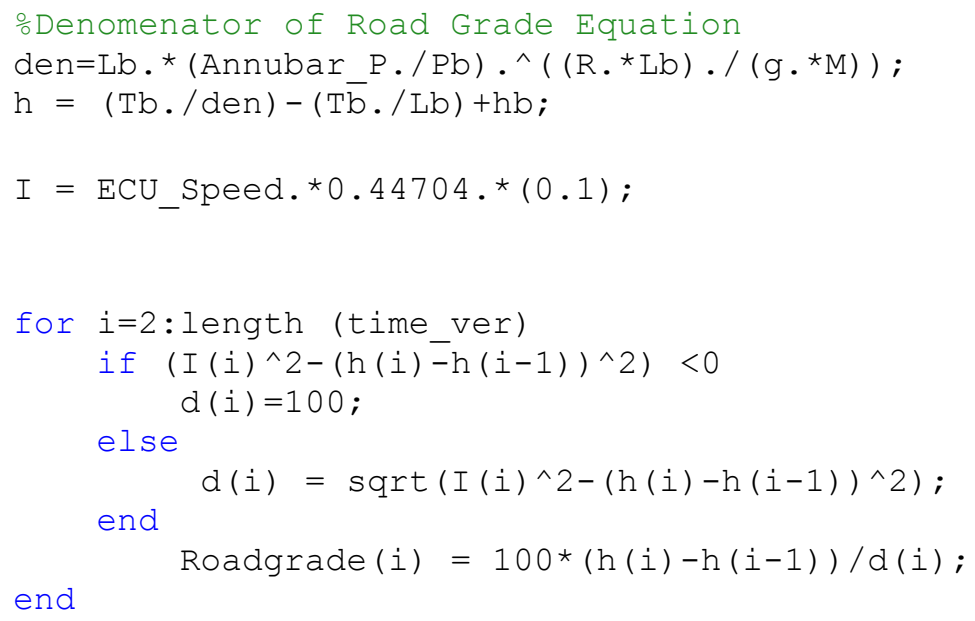




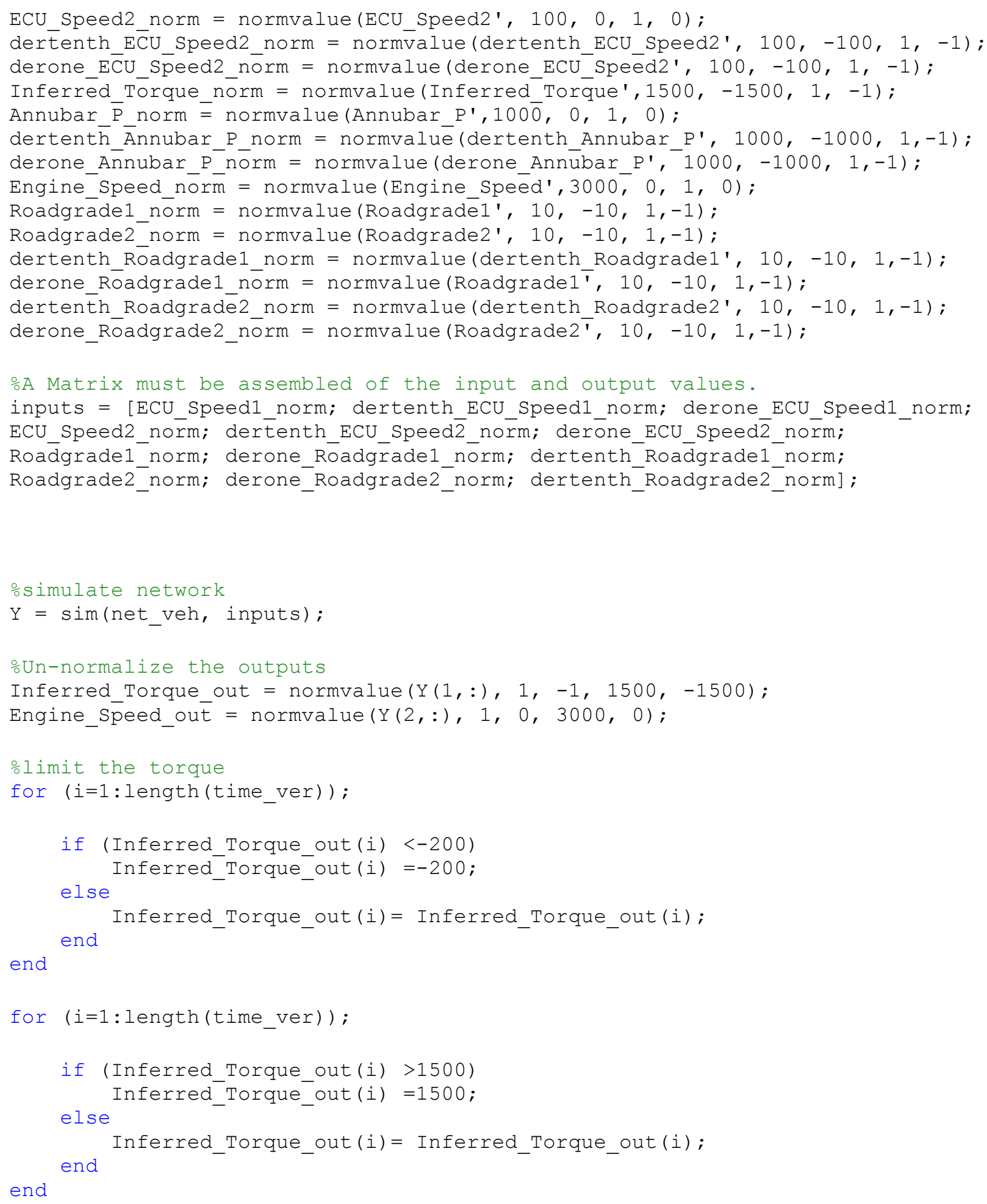


xlabel ('Time (secs)');

ylabel ('Engine Torque (ft-lbs)');

axis ([0 max(time) -1000 2000]);

legend('Actual', 'Predicted') ;

figure

plot(time_ver, Engine_Speed, '-b', time_ver, Engine_Speed_out, '--r'); xlabel ('Tíme (secs)') ;

ylabel ('Engine Speed (RPM) ');

axis ([0 max (time) -1000 3000]);

legend ('Actual', 'Predicted'); 


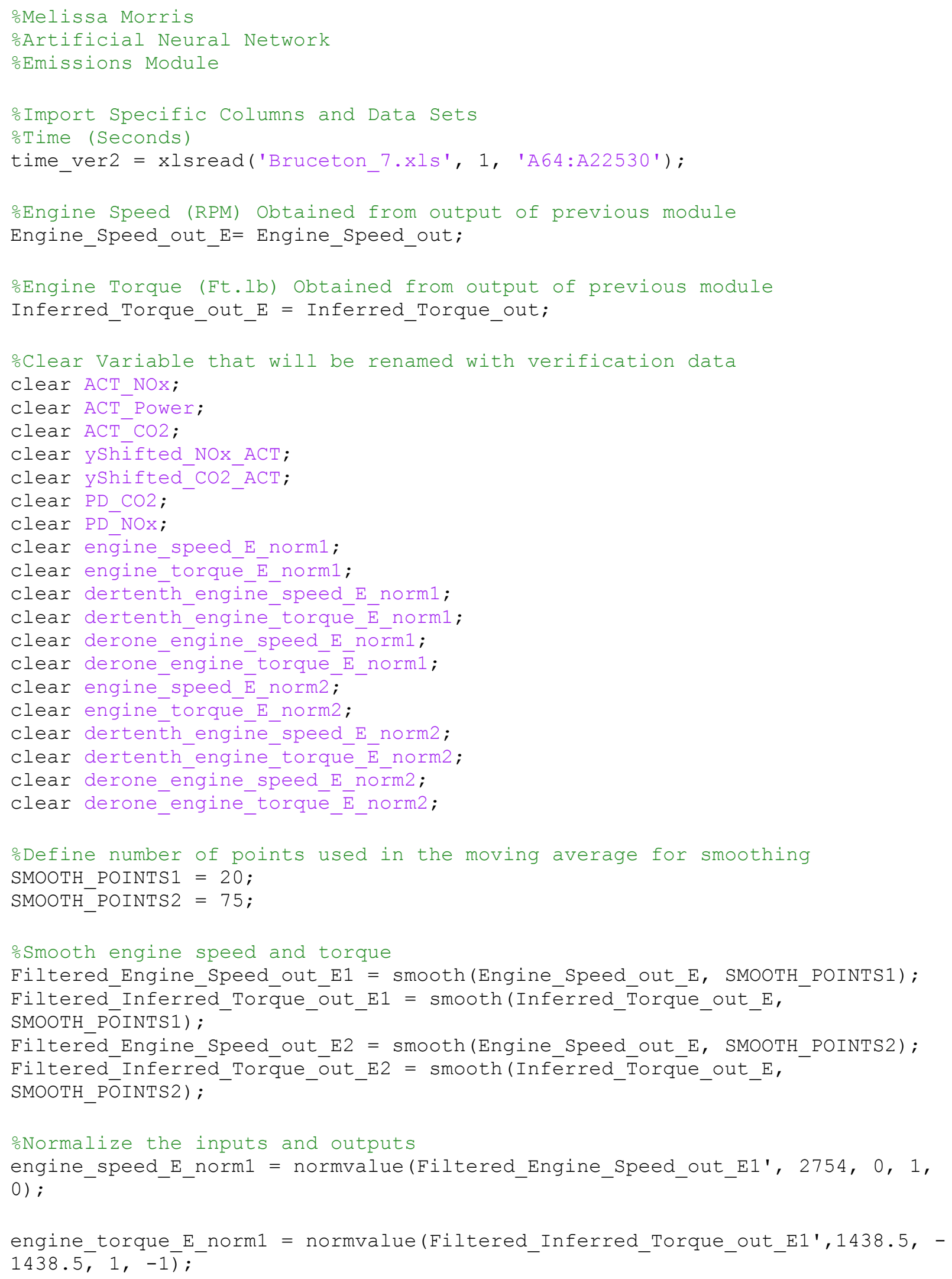




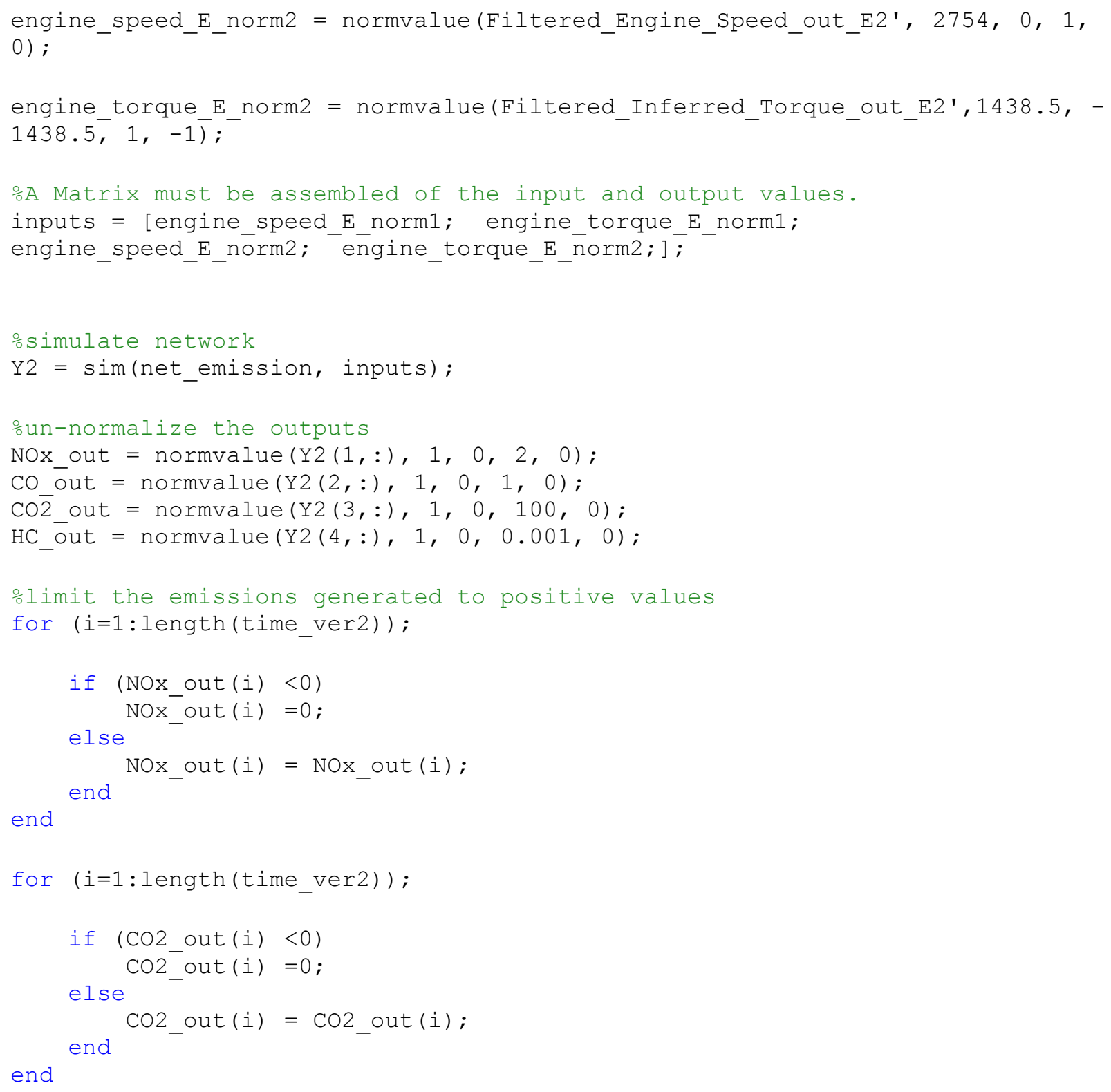




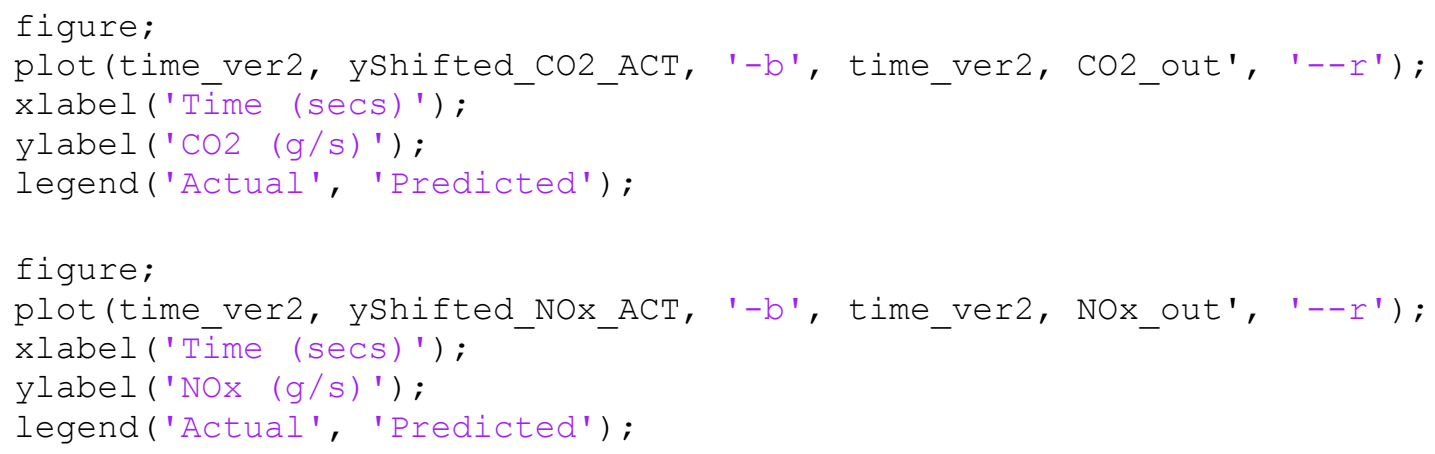

